University of Warwick institutional repository: http://go.warwick.ac.uk/wrap

A Thesis Submitted for the Degree of PhD at the University of Warwick

http://go.warwick.ac.uk/wrap/3032

This thesis is made available online and is protected by original copyright.

Please scroll down to view the document itself.

Please refer to the repository record for this item for information to help you to cite it. Our policy information is available from the repository home page. 
University of Warwick institutional repository: http://go.warwick.ac.uk/wrap

A Thesis Submitted for the Degree of PhD at the University of Warwick

http://go.warwick.ac.uk/wrap/3032

This thesis is made available online and is protected by original copyright.

Please scroll down to view the document itself.

Please refer to the repository record for this item for information to help you to cite it. Our policy information is available from the repository home page. 


\title{
AID PARTNERSHIPS AND LEARNING: UK AND JAPANESE PROJECTS IN GHANA
}

\author{
by \\ Chie Takahashi
}

A thesis submitted in partial fulfilment of the requirements for the degree of Doctor of Philosophy in Continuing Education

University of Warwick, School of Health and Social Studies

December 2002 


\section{Table of Contents}

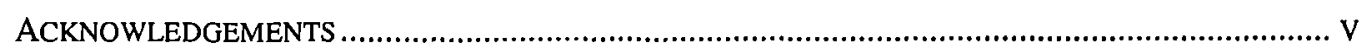

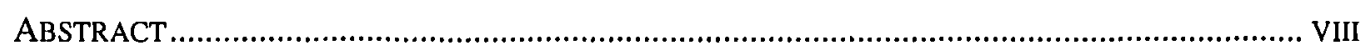

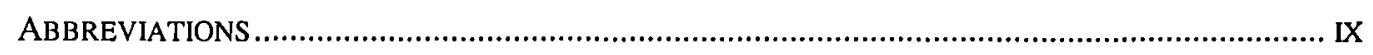

GLOSSARY OF KEY TERMS AND ORGANISATIONS ..................................................... XI

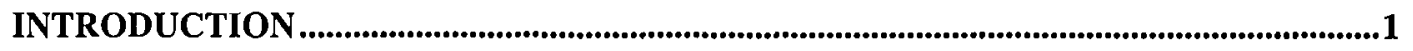

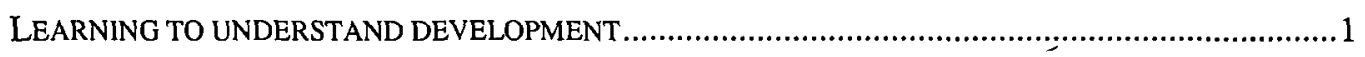

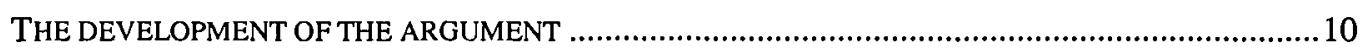

CHAPTER 1 PARTNERSHIPS: MEANINGS, PRACTICES AND COMPLEXITIES .....15

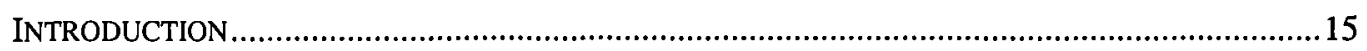

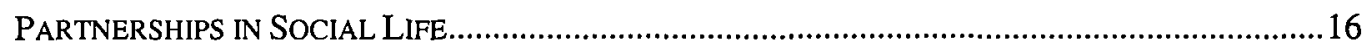

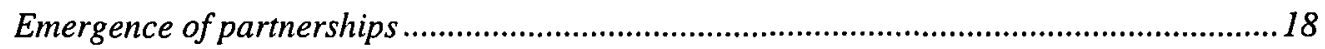

Co-ordination, networks and partnerships .................................................................20

PARTNERSHIPS AND INTER-ORGANISATIONAL RELATIONSHIPS ............................................28

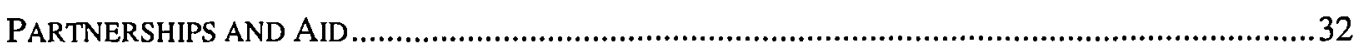

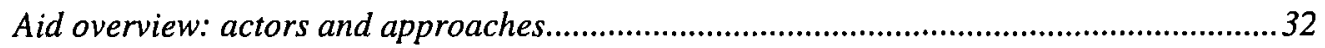

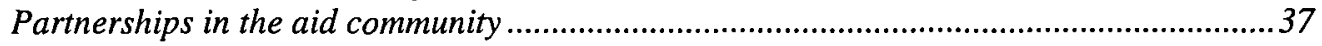

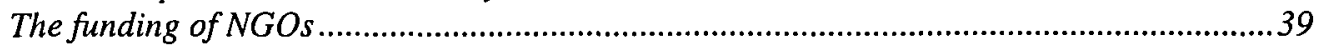

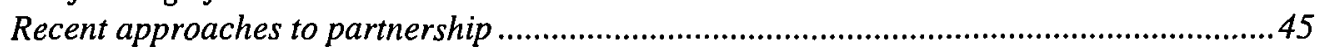

Critiques of development partnership.....................................................................46

Studies of inter-organisational relationships in aid partnerships................................. 48

CHAPTER 2 LEARNING AND ORGANISATIONS FOR PARTNERSHIPS....................54

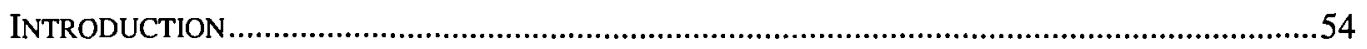

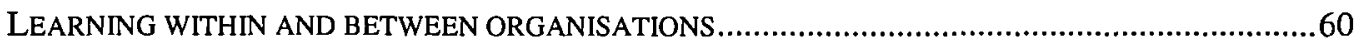

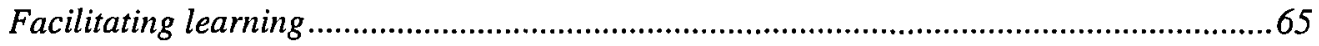

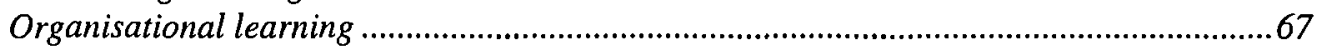

Relations and Learning in Organisations .....................................................................70

Relationships and Learning between Organisations ....................................................73

Exploitation of mutual learning in partnership ..........................................................76

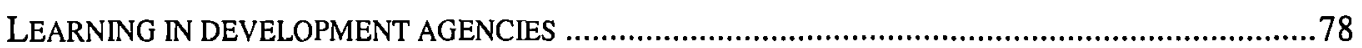

Practices of learning in development co-operation ....................................................81

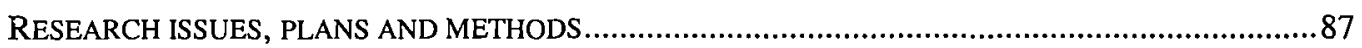

CHAPTER 3 DEVELOPING THE FIELD RESEARCH: BACKGROUND TO THE

CASE STUDY PARTNERS ................................................................................................90

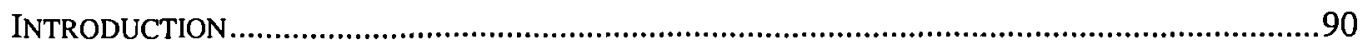

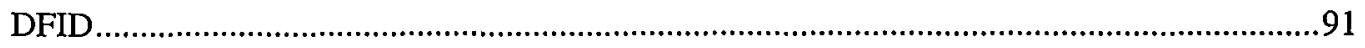

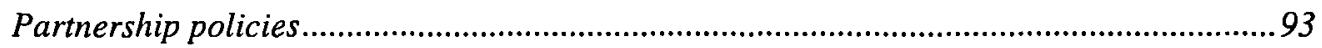

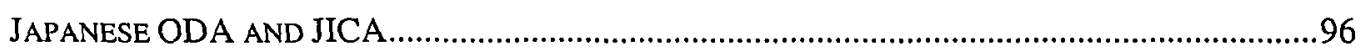

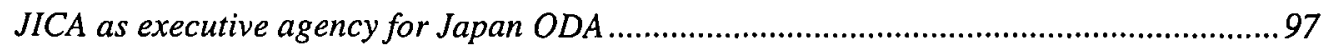

Japanese ODA: Partnership policy with NGOs...........................................................99 


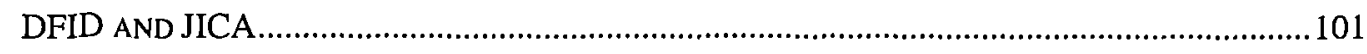

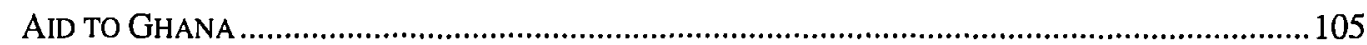

Case study projects and their organisational contexts ...................................................109

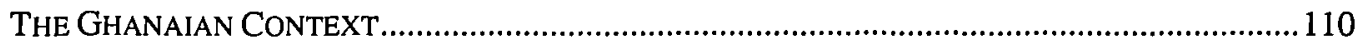

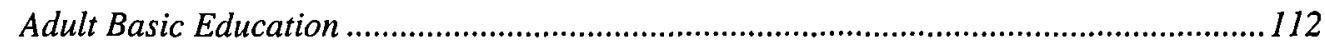

Maternity and Child Health ........................................................................................ 113

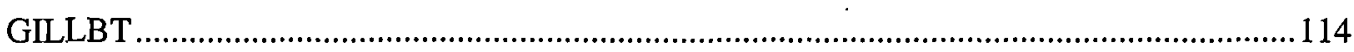

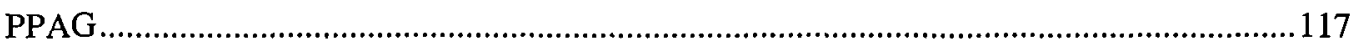

Critiques of family planning, literacy and income-generating activities...........................120

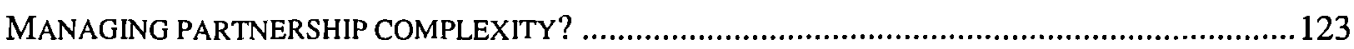

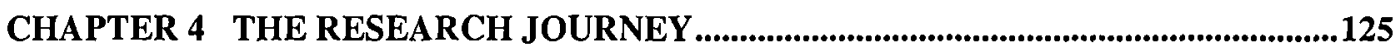

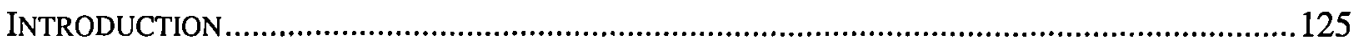

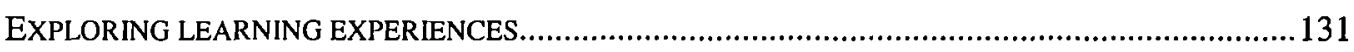

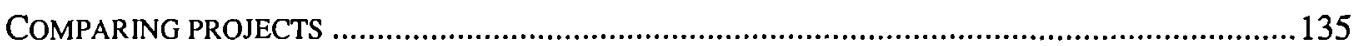

Field enquiry and subsequent analysis ..........................................................................136

MULTIPLE LINES OF INQUIRY: MANAGEMENT AND IMPLEMENTATION .....................................139

The quality of the data ...................................................................................................... 142

Communication tools: interviews and language..............................................................144

PROCESSING AND CONCEPTUALISING DATA........................................................................... 147

MY RESEARCH JOURNEY: REFLECTION ON THE INQUIRY......................................................150

CHAPTER 5 ANALYSIS OF INTER-ORGANISATIONAL RELATIONSHIPS ............154

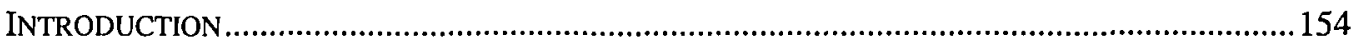

DFID funded adult literacy programme ............................................................................. 154

JICA funded maternity and child primary health care ………......................................... 156

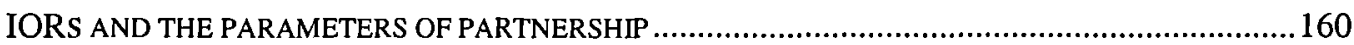

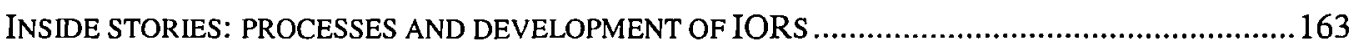

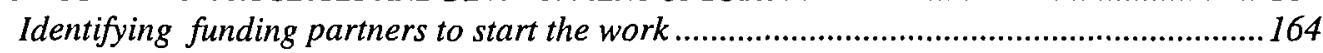

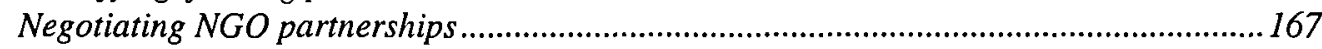

Inflexible implementation ……………….......................................................................171

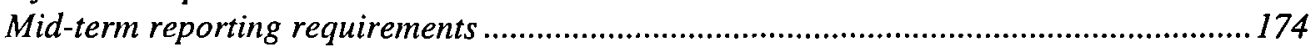

Knowing each other at distance.....................................................................................176

Obstacles to effective partner relationships..................................................................178

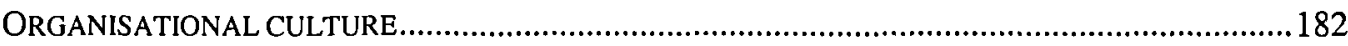

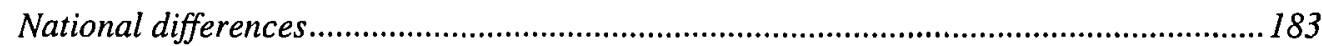

Organisational differences: NGOs and governmental organisations..............................184

Evangelical organisations .............................................................................................185

Personal capacity and linkages ....................................................................................186

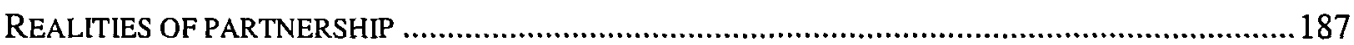

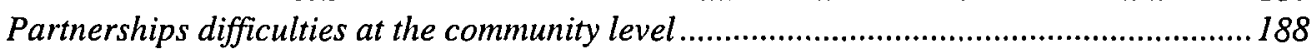

CONCLUSION: COMPARATIVE VALIDITY OF OBSERVATIONS...................................................191

CHAPTER 6 INTER-ORGANISATIONAL RELATIONSHIPS AND LEARNING.........193

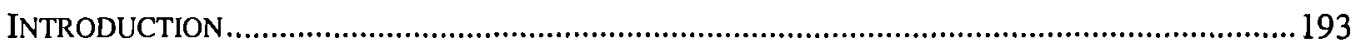

RESOLVING DILEMMAS BETWEEN ORGANISATIONS: TWO APPROACHES ....................................195

Learning to co-operate with principals and agents .........................................................196

Mutual learning to reduce hierarchy: the case of PPAG and JICA (Ghana) ....................200 
Maintaining the hierarchy: the case of GILLBT and DFID ............................................ 201

CO-OPERATING TO LEARN .................................................................................................2203

The relationships between NGOs: experiences in collaboration with NFED ................... 207

GILLBT's technical collaboration with Action Aid (Ghana) .......................................... 214

Rigidity or flexibility for changes .................................................................................... 221

LEARNING AND RELATIONSHIPS IN THE COMMUNITY .......................................................223

Gender relations: hierarchy and network at community level ...........................................223

Hierarchy in communities: the elder and chief, blocking or driving force?......................229

Managing community hierarchy.......................................................................................... 232

Getting to understand each other: new concepts in the same world? ............................... 235

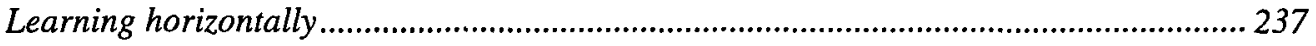

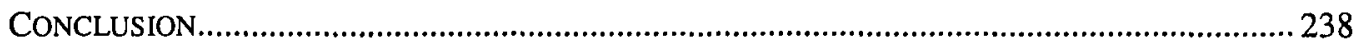

CHAPTER 7 LEARNING IN PARTNERSHIPS.................................................................242

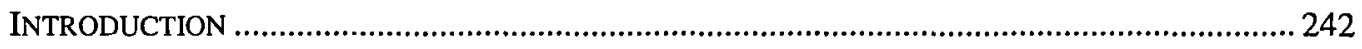

LEARNING FROM INTER-ORGANISATIONAL RELATIONS ......................................................... 244

Recipients' learning from donor requirements............................................................... 244

Donors' experiences of learning through commitment ....................................................... 250

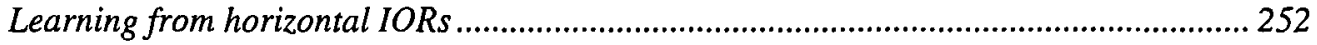

THE IMPACT OF INDIVIDUALS ON THE LINK BETWEEN IORS AND IOL ...................................25

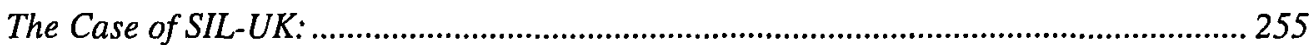

The Case of JICA (Ghana) .................................................................................................. 257

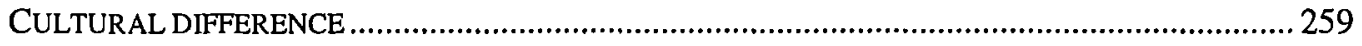

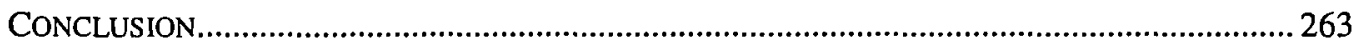

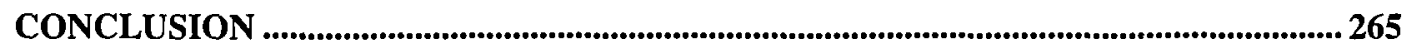

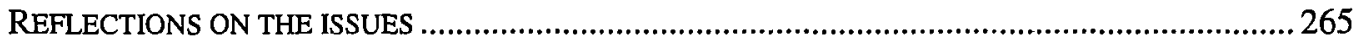

Approaches to the study .................................................................................................. 266

Discussion of individual versus organisational dynamics................................................. 268

CONTRIBUTIONS OF MY STUDY TO AID AND DEVELOPMENT .................................................22

REFLECTIONS ARISING FROM THE STUDY: SUGGESTIONS AND DIFFICULTIES .............................2. 274

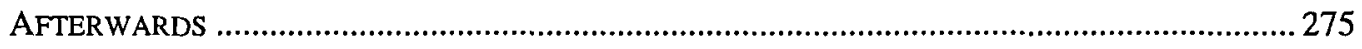

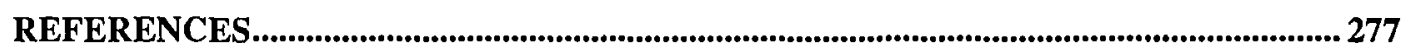

APPENDICES ............................................................................................................................................ 295

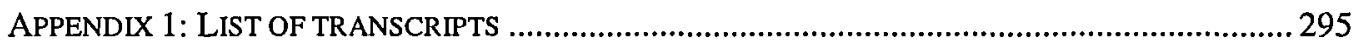

APPENDIX 2 UNPUBLISHED DOCUMENTS OF CASE STUDIES.................................................. 298

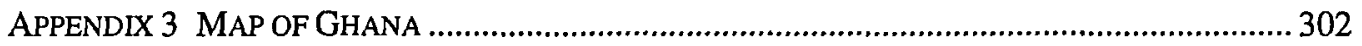

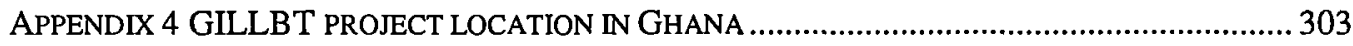

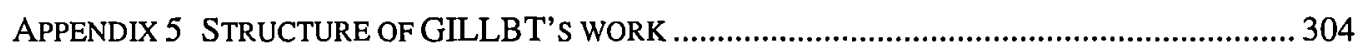

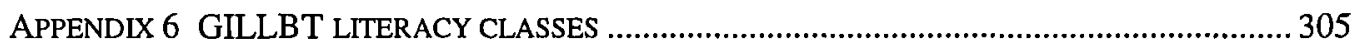

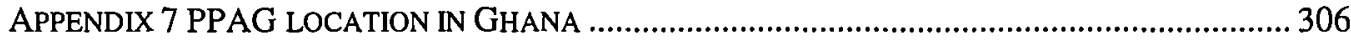

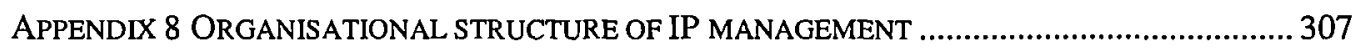

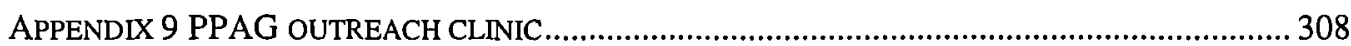




\section{LIST OF FIGURES AND TABLES}

$\begin{array}{lll}\text { Figure } 1 & \text { Major actors in aid delivery } & 37\end{array}$

$\begin{array}{lll}\text { Figure } 2 \text { The aid system hierarchy and networks } & 44\end{array}$

Figure 3 Hypothesised process of IOR and IOL 58

Figure 4 Positioning case studies in relation to IOR, IOL and partnership $\quad 59$

$\begin{array}{lll}\text { Figure } 5 & \text { Jarvis's learning model } & 63\end{array}$

$\begin{array}{lll}\text { Figure } 6 & \text { Process of gaining access to the case organisations } & 138\end{array}$

$\begin{array}{lll}\text { Figure } 7 & \text { Processes of overall data analysis } & 148\end{array}$

$\begin{array}{lll}\text { Figure 8a The DFID project } & 159\end{array}$

$\begin{array}{lll}\text { Figure 8b The JICA project } & 159\end{array}$

Figure 9 Modified principal and agent theory: effects of learning 199

$\begin{array}{lll}\text { Table } 1 & \text { Main approaches to learning and assumptions } & 62\end{array}$

Table 2 Personal and organisational influences on learning 69

$\begin{array}{lll}\text { Table } 3 & \text { Actors in the project case studies } & 160\end{array}$

Table 4 IORs in two case study projects 161

Table 5 A contrast between rhetoric and reality of IORs 190

Table 6 The basic idea of the two prisoners' dilemma 205

Table 7 The NGOs' dilemmas and its IOL consequences 206

Table 8 Examples of IORs and IOL with GILLBT and Action Aid 219 (Ghana) 


\section{Acknowledgements}

This thesis owes its value to a number of people. Without their contributions and support anything could have been barely written in this work.

For the inspiration and the development of the thesis, I wish to express my deepest gratitude to Rosemary Preston who stimulated me to start and supervised my $\mathrm{PhD}$ study. Her intellectual insights and enthusiasm into the study of development and education have promoted my entire research journey at Warwick. I cannot thank her enough for her considerable and unremitting support for both my study and my stay at Warwick.

I am indebted to almost a hundred people who have given their time and efforts to this thesis. Without their willingness to share their knowledge, it would have not been possible to develop the argument on which this thesis is based. They include staff at: UK Department for International Development (London, East Kilbride and Accra); Ghana Institute of Linguistics, Language and Bible Translation (Tamale and Accra); Japan International Cooperation Agency (Tokyo, London and Accra); Plan Parenthood Association of Ghana (Accra).

My special thanks go to many Ghanaians who accepted me warmly and helped me to conduct my research in their villages by telling me their stories and showing me their work.

I am very grateful to Lalage Bown and Clinton Robinson, who facilitated my access to Ghanaian communities ensuring the success of my stay. My warm thanks go to Frances Ademola who made my stay in Accra most comfortable at her house and shared her valuable perceptions of her country.

I must acknowledge Japan Foundation Endowment Committee for sponsoring all my fieldwork. 
I benefited from discussions with interested groups throughout the process of the study. I would like to thank individuals and participants at several conferences: the BAICE conference at Edinburgh 2001; INCED conferences at Warwick in 2001 and 2002.

For editing and proof reading of the thesis, I would like to thank Gerard Sharpling and Beth Wishart.

I have appreciated the kindness of my friends not only at Warwick but also elsewhere. They have helped me to sustain my motivation at different stages in this PhD. Especially I would like to thank Lin-tzy Liou and Wen-Bing Gau with whom I spent valuable time in the research student room. I will always treasure their generous intellectual, technical and emotional support.

I am truly grateful to Giovanni Ganelli who willingly gave me help, strength and encouragement to release any difficulties that arose in the period of the study.

Finally I would like to thank my parents, Isao and Kimiko Takahashi, my brothers, Hidehisa and Fumimasa, who have maintained their belief in me completing this work. Without their tremendous understanding, trust and support, I would not have had the opportunity to conduct the research. 


\section{Declaration}

I declare that this thesis is my own work and that it has not been submitted for a degree at another university. 


\section{Abstract}

International technical assistance today supports pro-poor intervention managed and implemented by a number of organisations working in partnership together located in several countries. They may include funding organisations, governments, non-governmental organisations and community groups. This thesis explores the meaning of aid sector partnership and some of the ways in which they work to support community development in Africa. The study is of the ways in which partners interact and learn from each other, the contextual issues that influence the process and the implication of this for what is achieved.

Believed to be the first of its kind, the study compares two bilaterally funded projects implemented by Ghanaian NGO counterparts. The British Department for International Development (DFID) financed an adult literacy project in the North, while Japan International Co-operation Agency (JICA) supported a maternal and child health care project in the East of Ghana. The study examines the quality of relations between partners in the two projects and then the ways in which these are informed by incidental learning experiences. A fieldwork was conducted in Ghana, UK and Japan. Data are largely narrative derived from in-depth interviews with more than 100 informants. Critical incident analysis is employed as the main interpretative strategy.

The thesis conceptualises instances of inter-organisational learning (IOL) in terms of theories of principals and agents, prisoners' dilemmas and women's place in community development. It shows (i) that IOL can be used to maintain and modify relations of control and dominance in partnership hierarchies, and (ii) that IOL serves as a by-product of horizontal relationships and be increased or reduced in the competition between partners for resources and identity. The influential role of individuals, beyond the boundaries of organisations is stressed through social networks and trust-based relations, as are instances of resistance to learning as a consequences of personal conflict. However, structural constraints in the aid system, as demonstrated by asymmetric access to resources, expertise, knowledge, status and networks, ultimately determine the quality of funding management schemes and an environment that stimulates mutual individual learning, which is advantageous circumstances may lead to organisational learning and inter-organisational learning. 


\section{Abbreviations}

$\begin{array}{ll}\text { BOND } & \text { British Overseas NGOs for Development } \\ \text { CBD } & \text { Community Based Distribution } \\ \text { CBO } & \text { Community Based Organisation } \\ \text { CDF } & \text { Comprehensive Development Framework } \\ \text { CEP } & \text { Community Empowerment Programme, JCA } \\ \text { CIDA } & \text { Canadian International Development Agency } \\ \text { CSD } & \text { Civil Society Department, DFID } \\ \text { CSCF } & \text { Civil Society Challenge Fund, DFID } \\ \text { DAC } & \text { Development Assistance Committee, OECD } \\ \text { DFID } & \text { UK Department for International Development } \\ \text { GDN } & \text { Global Development Network } \\ \text { GILLBT } & \text { Ghana Institute of Linguistics, Literacy and Bible Translation } \\ \text { HIV/AIDS } & \text { Human Immuno Virus/ Acquired Immune Deficiency Syndrome/ } \\ \text { IGAs } & \text { Income Generating Activities } \\ \text { IMF } & \text { International Monetary Fund } \\ \text { INGOs } & \text { International NGOs } \\ \text { INTRAC } & \text { International NGO Training and Research Centre } \\ \text { IOL } & \text { Inter-organisational learning } \\ \text { IORs } & \text { Inter-organisational relationships } \\ \text { IP } & \text { Integrated Family Planning, Nutrition and Parasite Control } \\ \text { IPPF } & \text { International Planned Parenthood Federation } \\ \text { JICA } & \text { Japan International Cooperation Agency } \\ \text { JOICFP } & \text { Japanese Organisation for International Co-operation in Family } \\ \text { JODA } & \text { Jlanning } \\ \text { LNGOs } & \text { Local NGOs } \\ \text { MOFA } & \text { Ministry of Foreign Affairs, Japan } \\ \text { NFD } & \text { Non-Formal Education Division, Government of Ghana } \\ & \end{array}$


NGO Non-Governmental Organisation

ODA Official Development Assistance

OECD Organization for Economic Co-operation and Development

PALS Participatory Approaches Learning Study

PPAG Planned Parenthood Association of Ghana

PRSP Poverty Reduction Strategy Papers

REFLECT Regenerated Freirean Literacy through Empowering Community Techniques

SAPs Structural Adjustment Programmes

SAT Sinapi Aba Trust

SIDA Swedish International Development Cooperation Agency

SIL Summer Institute of Linguistics

SWAPs Sector-Wide Approaches

UN United Nations

USAID United States Agency for International Development 


\section{Glossary of Key Terms and Organisations}

Specific terms used in this study are summarised below, drawing largely on the ways in which they are described in official documents. Other terms being used in ways particular to this thesis conceptualised by the author from definitions used in relevant literature.

\section{Action Aid (Ghana)}

Ghanaian NGO, a branch of Action Aid operating since 1990. Action Aid is one of the UK's largest development NGOs, working in over 30 countries to eradicate poverty since its foundation in 1972. Action Aid (Ghana) supports the basic needs and rights of poor people to improve their access to services and to lobby government. For adult literacy, it uses the REFLECT methodology. Action Aid (Ghana) is based in Accra and there is a support office in Tamale.

\section{Bilateral aid}

A classification of ODA referring to aid implemented between a donor and recipient country.

\section{Capacity-building}

An ongoing process of helping people, organisations and societies to improve and to adapt to change around them. Performance and improvements are governed by the missions, objectives, contexts, resources and sustainability of those involved. Organisational capacity-building is a conscious intervention to improve an organisation's effectiveness and sustainability, in relation to its mission and context (INTRAC definition)

\section{Development Assistance Committee (DAC)}

Founded in 1961, DAC is a subordinate agency of the Organisation for Economic Cooperation and Development, OECD. DAC examines aid policy development and member countries' implementation of aid funded projects. DAC collects statistics on the state of economic development and aid conditions in recipient countries 
(JICA1998).

\section{Department for International Development (DFID), UK}

The UK government department responsible for promoting development and the reduction of poverty. Created in 1997. It was formerly the Overseas Development Administration, ODA.

\section{The Ghana Institute of Linguistics, Literacy and Bible Translation (GILLBT)}

A non-government organisation working for the well-being of Ghanaians by providing literacy programmes, linguistics research and the translation of the Bible into mother languages. It started as a branch of SL in 1962, recognised as an NGO in 1977 and was handed over to Ghanaians in 1980. Head quarters located in Tamale.

\section{Income Generating Activities (IGA)}

Helping the poor by providing loans with small low interest loan rates in order to set up sustainable economic activity, by selling goods and services so as to engage in the market.

\section{Inter-organisational learning (IOL)}

Organisational changes consequent upon individual interactions beyond boundaries of individual organisations, informally and formally.

\section{International Planned Parenthood Federation (IPPF)}

The largest international voluntary organisation in the field of sexual and reproductive health including family planning. Links with national Family Planning Associations in over 180 countries worldwide. Registered as a charity in the UK.

\section{Japan International Cooperation Agency (JICA)}

An agency for the management and implementation of Japanese technical assistance. It is an executive agency for Japanese ODA and affiliated to the Ministry of Foreign Affairs. Established in 1974. 


\section{(Adult) Learning}

Learning is a contested term in many conceptualisations. In this thesis adult learning refers to both the acquisition of knowledge and to the consequent changes in behaviour and underlying values that result from it. This is consistent with the view of Brundage and Machkercher (1980:5), who define adult learning as the process through which individuals go as they attempt to change or enrich their knowledge, values, skills or strategies and as the resulting knowledge, values, skills, strategies and behaviour possessed by each individual.

\section{Low income countries}

A classification of countries according to income used by the DAC of the OECD. In 1998 , it referred in principle to countries with per capita GNP $\$ 760$ or less. (OECD 2000)

\section{Multilateral aid}

ODA involving government and regional co-operation in the provision of funds to the World Bank and other international organisations for purposes of international development.

\section{Network}

A form of co-ordination in social life replacing vertical control such as the market and authority. Composed of a unit of organisation and/or individual based on common values and/interests, informally and formally.

\section{Official Development Assistance (ODA)}

ODA comprises funding flows which meet the following three conditions. (i) It is provided by official agencies or their executive agencies. (ii) It is administered to promote the economic development and welfare of developing countries as its main objective. (iii) It is concessional in character and conveys a grant element of at least 25 percent. ODA consists of grant aid, technical cooperation, subscriptions and contributions to the U.N. agencies and international financial institutions and government loans (OECD) 


\section{Organisational learning}

Referring to 1) incremental changes that correct mismatches between intentions and what actually happens by changing organisational behaviour and actions; and 2) radical changes in the values and assumptions that underpin organisational goals, after correcting mismatches.

\section{Partnerships}

In this thesis, partnerships refer to any forms of inter-organisational relationships arranged to achieve common goals through joint effort. In policy rhetoric, the concept of partnership may imply equitable access to resources and status in decision-making between partners. This contrasts with contemporary interpretations of partnership as political and economic expedient for efficient aid management and continued inequality between partners.

\section{The Planned Parenthood Association of Ghana (PPAG)}

Ghanaian NGO providing family planning services, education and advocacy in sexual and reproductive health and responsible parenthood. Founded in 1967 and affiliated to IPPF in 1969. Head quarters located in Accra.

\section{Project cycle}

The series of stages undertaken in the planning and implementation of a development project. It starts with the initial idea for a project and concludes with the completion of the implementation phase and the evaluation of the project's results. The stages of the project cycles are referred to differently by different donors, but the following are widely used terms: identification; design; appraisal; approval; implementation and evaluation (ODA 1995).

\section{Sinapi Aba Trust (SAT)}

Ghanaian NGO offering to serve opportunities for enterprise development and income generation by providing credit support to the economically disadvantaged to transform their lives. Established in 1994 as one of the implementing partners of the Opportunity International Network based in Chicago, US. 


\section{Sector wide approach (SWAP)/ reform}

Designed to support policy reform across a particular area of government activity such as education and health, and can be a combination of different types of support. Frequently required as a conditionality of IMF and the World Bank loans. It aims to help improve the long-term performance and viability of the assisted sector through market oriented decentralisation and privatisation of former state function.

\section{The Summer Institute of Linguistics (SIL), UK}

UK NGO. A branch of SIL International, which works for language development, literacy, translation, research and training in 70 countries. Its members share a Christian commitment. Its parent body is in Dallas, USA. 


\section{INTRODUCTION}

In this Introduction I shall describe my research journey, and refer to some aspects of the processes which have led me to develop the questions investigated in the thesis. First, I shall present two accounts based on personal experience. Then I shall explain how they stimulated my research in a somewhat instinctive way. Following this, I shall refer to my academic reading and field exercise, both of which inspired my analysis, whilst at the same time consolidating the ideas on which to focus. Lastly, I shall provide a synthesis of the chapters to come.

\section{Learning to understand development}

My involvement in development studies is rooted in my first visit to the Philippines in 1995 during a study tour organised by a Japanese Nongovernmental organisation (NGO), when I was at a university in Tokyo completing a degree in English Literature. After the study tour, I started to work as a volunteer at the NGO for several years until my graduation from the University. By the time I graduated, I decided to commit myself to learning more about the issues related to poverty and international development aid. After completing an MA in Development Studies at the University of Leeds, I enrolled as a PhD student at Warwick.

One particular scene led me to research the patterns of relationships between the 
players in international development services. This occurred when I was working as a member of an NGO committed to supporting street children in the Philippines.

A middle-aged man from the midst of a crowded auditorium was arguing passionately about funding procedures with some Ministry representatives who were sitting far away from him on the stage. The scene kept coming back to me five years after the event, partly because of the disappointment and confusion on the man's face, but also because of the effect of his words on other people in the audience. I cannot recall exactly what he asked or how it was answered. Like him and others, I experienced a sharp sense of shock at discovering the ignorance and lack of interest expressed by the funding representative at the man's concerns. I was also uncomfortable in observing the wide separation between the stage and the audience, both physically and psychologically, not least because the theme of the forum was "Creating together a new Partnership: NGO support schemes contributing to people's self-reliance" (JANIC, 1996).

My second experience was also in the Philippines. I was talking to a local volunteer who was working with rubbish scavengers. She conveyed a total loss of faith in the national government intervening to solve the social and economic problems that people face. The scavengers are people who survive by collecting refuse in order to exchange it for cash. They often live in or near a dump in a city, where they are excluded from social benefits and welfare systems, despite their extremely difficult conditions. The government had done very little to improve their lives. I can never forget a pregnant woman carrying a baby in a house, which was made of pieces of rubbish, while small children ran along a 
track throwing rubbish, adding to the mountains of waste. The picture stays in my mind together with an inexpressibly strong smell.

My personal experience of working in the capacity of a volunteer for a Japanese NGO, as well as my observation of international and local social workers in the Philippines, gradually led me to become more interested in the question of the quality of the relationship between NGOs and governments at global, national and local levels. The gaps between the experiences of people working in the field and the perceptions of policy makers revealed in the above examples from my personal experiences relate to a lack of mutual understanding and commitment between the various organisations acting in international development. Problems are unavoidable when so many different organisations, such as funding bodies, managing and implementing agencies, have to work closely together for protracted periods.

With such thoughts, and anxious to know more about how the system worked, I decided to prepare for an MA in Development Studies at the University of Leeds in the UK. I gained an understanding of some theories of development, substantive issues of development, development strategy and project appraisal with my particular interests in social development. My MA dissertation was a comparative study of bilateral aid organisations' policies and strategies for basic education. It looked at donor approaches to primary education and their relevance to recipients' needs. It argued that prioritisation was given more to donors benefits and aid policies than to what the recipient required. I saw the 
collaboration and the sharing of information between donors and recipients as being essential to fulfilling recipients' needs. My MA dissertation argued for the importance of building close relationships between governments, including NGOs, since NGOs are often based in local situations with good community knowledge and expertise, particularly in education.

From here, I wanted to continue my studies in development in depth. I thus started what has become a four-year research programme at the University of Warwick. Here, I became even more passionate about development aid issues and processes. During my time at Warwick, a series of experiences have led me to understand more about the organisational complexity of the aid system and to look into the work of actors, besides governments, and the relations between them.

At the end of the first year of my $\mathrm{PhD}$, I conducted a review of a research project entitled 'The Participatory Approach Learning Study' (PALS). The UK Department for International Development (DFID) had contracted this study in 1996 to an NGO support organisation, the International NGO Training and Research Centre (INTRAC). The purpose of the study was to evaluate the meaning of 'participation' in six DFID funded projects that had been completed in 1996 to 1998 in the countries of India, Bangladesh, Nepal, Ghana, Egypt and Nigeria (INTRAC, 1999). As such, it was a secondary study. The primary aim was for DFID to improve its application of participatory approaches by analysing the projects as they have been implemented in six countries across multiple 
sectors, gaining perspectives from DFID staff and relevant project partners, including primary and secondary stakeholders.

From interviewing researchers in INTRAC, I learned that the process of conducting research into aid sector processes mirrors, in effect, the complications of aid delivery mechanisms and services when they involve various stakeholders. Those reviewing the PALS study found that there were misinterpretations of the research purpose and content, between DFID head quarters and DFID geographical offices, as well as between DFID and INTRAC, the project implementing organisation (INTRAC 1999). Some of the confusion arose between the organisations involved in the research because of their diverse perceptions of the term participation and partnership, and of the needs, objectives, and strategies of the evaluation. The original aim of the study was to identify DFD staff learning and training needs to increase awareness of participatory methods and strengthen participatory approaches in DFID funded projects. For this, the study highlighted the fact that project implementers and users may not consider participation to be a key element in the implementation. The PALS research again reflected my feeling that the systems, procedures and structures of the overall framework of the funding organisation, in this case DFID, are far more important.

The PALS research taught me about some of the problems faced by funding, managing and implementing organisations in achieving participatory approaches in the spirit of partnership. It also made me aware of the complexity and 
confusion inherent in projects involving several organisational actors trying to work in 'partnership'. The difficulties appear to be associated with a lack of mutual understanding throughout the project, as well as with inadequate information at the design stage.

During the second year of my $\mathrm{PhD}$, I conducted a study for the Japan International Cooperation Agency (JICA) regarding DFID's system of contracting-out of projects to UK NGOs. JICA is an executive agency for Japanese Overseas Development Assistance (ODA). Contracting-out, in DFID's case is a system, in which the NGOs tender to deliver the services of the UK government in different parts of the world. The study was intended to provide background information which could inform JICA's strategy for working with NGOs. The experience taught me about the historical shift in the relationships between the government, the managing and the implementing organisations and how they are affected by changes in policy. It provided me with insights into various schemes and their implications for relations between NGOs and the government. It also allowed me to gain knowledge of how funders and implementers perceive each other. Above all, the study gave me an unusual personal experience in that I was able to assist in facilitating JICA's learning about how DFID works with NGOs. I was already attracted by the idea of partnership. I then became interested in the idea of comparative case study analysis of human development assistance funded by each of the two institutions and the roles played by different organisations in their delivery. 
The topic of this thesis is timely for two reasons. First, there has been little or no comparison of bilateral funders at the meso level of project delivery processes. The timeliness of this research project may also be attributed to the fact that interest in "partnership" in the analysis of relationships between various kinds of organisations committed to international development has dramatically increased. Current debates on the discourse of partnership stimulated my inquiry about what partnership means, both rhetorically and in practice. For instance, from the observation of multiple organisational actors, with their different roles and their different positions in complex aid mechanisms, we can deduce that the reality faced by each organisational actor in such settings is not as straightforward as the word partnership implies. If partnership projects funded by two bilateral organisations from very different parts of the world were to form the basis of the proposed comparison, it would be quite complicated to analyse its findings. Yet, of principal concern to me is what partnership means in practice, and not simply in terms of the rhetoric of an attractive concept.

The next question to be addressed is how to undertake such a study and plan its key elements: its middle range questions and approaches to data collection and analysis, given that the aid system is a complex network of organisations across the world. Partnership in the aid sector refers both to a pattern of relationships between organisations and the ways in which they try to achieve common goals. The challenge is how to investigate the nature and processes of the relationships between organisations sufficiently clearly to reveal dimensions of partnerships as they develop their practice. Central to this research is how the term used by 
various organisational actors in the aid sector may be understood and challenged by the individuals involved.

My inquiry starts by examining the general nature of relationships between organisations situated within a network, before considering how they might be different in the aid sector. Within this, there is a need to take account of how the status of organisational actors and their members influence what occurs between them. Next, there is a case for examining how and why these patterns vary as a project develops. Finally there is the question of the implications of these arrangements for organisational collaboration and for the outcomes of the development work. In questioning what happens between collaborating actors in the aid sector, I explore the issues and factors that determine the characteristics of inter-organisational relationships (IORs). Since these evolve over time, there is a case for seeking to understand the dynamics of the process. I suggest that one of the crucial factors involved in changing IORs is first how actors learn from each other and then how such learning influences their further interaction.

The study sets off to understand in depth about the international aid system as a driving force of development projects. I have chosen to do an exploratory study. I propose comparing two case study projects to examine the commonalities of their partnership experiences the extent to which they may be replicable in respect of their micro level processes. Broadfoot (1999) states that comparative studies build on systematic studies of common issues, needs, or practices as they are realised in diverse cultural settings. This enhances awareness of possibilities, 
clarifies contextual constraints and contributes to the development of a comprehensive socio-cultural perspective. As for the ways in which these studies are conducted, Titmus (1989) points out that many of the comparative studies are descriptive juxtapositions and only few identify differences and similarities between phenomena. The complexities of comparison are recognised by many writers. Relevant to this thesis, they include: indicating awareness of local cultures in globally constructed contexts (Watson 2001); how to represent the culture of organisations and how it influences the ways in which they respond to issues of concern (Ouch and Wilkins 1998).

Many layers of analysis are possible in conducting comparative research. Broadfoot (1999:26) claims that comparative studies highlight "the multiple levels of cultural perspective and ideological and institutional constraints that constitute the territory for social science". As I began to investigate my research questions, I realised that I would be dealing with the multiple perspectives that significantly influence the analysis of aid partnerships issues. I would be comparing organisations of different statuses in organisational hierarchies and examining how their interaction affected the overall partnership endeavour. I knew that I was being led towards an analysis of organisational learning as a central field of my comparative analysis. I was also interested in examining the phenomena from the perspectives of aid system and multiple aspects of culture. In doing so, the comparative study reduces the isolation of the case from global situation of aid system. This prevents the research from being seen as fragmented and disconnected. 


\section{The development of the argument}

The starting point of my inquiry is the conceptualisation of partnership. This is to help my understanding of the diverse patterns and practices of partnership, before moving to examine two aid project case studies. Organisational partnerships are examined in Chapter 1 so as to prepare the way for the interorganisational analysis that follows in the later discussion. An investigation of the literature on inter-organisational relationships is carried out to explore the definitions and mechanisms of partnership. The idea of collaboration is used here as a means of understanding the nature of partnerships as a form of interorganisational relations. This is set against the nature of the competitive and hierarchical structures of such relations in society. Characteristics of networks are compared with those of partnerships, with reference to issues of resource and identity. The chapter then shifts to an examination of the reasoning behind the recent, increased reliance on partnership in general and a critical analysis of factors affecting IORs in partnership arrangements. The chapter then concentrates on these issues in the aid sector. The research undertaken highlights the complexity of the issues in relation to partnerships and IORs at various levels of the hierarchical structure, within the aid system. After introducing recent partnership policies and approaches, relations between government and NGOs are considered as they will be the central actors in this thesis. Learning between and within organisations is discussed both implicitly and explicitly as an

important parameter in the process of achieving collaboration in such studies. From here, I position my research as a new analysis focusing on learning between organisations in comparable projects, funded by different donor 
governments.

Chapter 2 explores the analysis of inter-organisational relationships in the previous chapter from the perception of learning. The growing interest in organisational learning is examined in terms of its mechanisms of facilitation and control, as well as in terms of individual and organisational determinants and theories of learning. Most of the literature has been developed with reference to the business sector, but I draw influences from this for the aid sector. Interorganisational relationships (IOR) and inter-organisational learning (IOL) are introduced from the perspective of how organisations are constructed and managed, and of how these elements impact on learning within and between organisations. Issues of control, such as the exploitation of partners in such processes, are carefully examined as parameters of organisational mechanisms and of inter-organisational relationships. They provide the framework for my research, which compares accounts of practices of different organisations and their networking with how they achieve collaboration in delivering aid projects.

Chapter 3 describes the structures and linkages between the organisations which were used in the case studies. It provides an overall picture of channels of resources, accountability, and information. The four main case study organisations are DFID and JICA as bilateral donor organisations, and the Ghana Institute of Linguistics, Literacy and Bible Translation (GILLBT) and the Planned Parenthood Association of Ghana (PPAG) as Ghanaian implementing NGOs. The organisational differences between DFID and JICA are examined, in 
order to indicate possible similarities and differences in the ways in which their funding projects are designed and implemented, in collaboration with local counterparts. Projects aiming to improve adult literacy, maternity and child primary health care are investigated in the Ghanaian context. This is followed by a consideration of the contributions made by the NGOs involved in the case studies, in terms of their expertise and the nature of their collaboration with other organisations in Ghana.

Chapter 4 introduces my accounts of the means of accessing data and its conceptualisation for the purposes of the study. As well as managing the organisation complexity, this process had physical constraints. It required travel between three countries; significant negotiation with gatekeepers, and a wide range of primary and secondary resources. Extensive reference to these elements is required in order to explain the quality of the data obtained in the two comparative case studies. The methods used to identify my research questions pertain to qualitative research and a case study approach, in which narrative and critical incident analysis are employed as the interpretative strategies. The processing analysis and presentation of data are also clarified, so as to indicate the development of the investigation. A parallel theme of the thesis is a reflection on how I learnt, as a researcher, outside the community, to gain the trust and confidence of the members of the organisations from whom I collected the data about their experience of their involvement in the projects.

Chapter 5 is concerned with the two case studies, and provides a particular focus 
on the dynamics of IOR at different project phases. It illustrates various types of IOR, often associated with partnership and co-operation. It further investigates what occurred on a day-to-day basis at various stages of the project delivery, and the determinant characteristics of each IOR parameter. IOR used in the case study analysis are structured differently, for example by means of hierarchical relationships and horizontally networked relationships. The analysis focuses on the gaps between what has been claimed in statements, such as policy, strategy and planning documents about partnerships and the forces for action in practice.

Chapter 6 provides a conceptualisation of my data on inter-organisational relationships and learning. This chapter uses management theories to conceptualise the selected case study IORs. These include hierarchical donorrecipient relationships; competitive government-NGO and NGO-NGO relationships. These structural relationships, examined in the light of theoretical approaches, provide a useful application of concepts of learning to the process of building different forms of IOR. From this, the implications of the relationship between learning and partnership are drawn. Down the project hierarchy, accounts of project implementation at field level reveal the struggles of those participating in and managing the projects, in terms of power relationships at the community level. Gender relations and the involvement of women in projects are also considered, along with women's learning experiences of tackling hierarchical relationships. Finally, reference is made to how the hierarchy of communities and the consequent difficulties of collaboration and mutual learning constrained the implementation of the two projects. 
Chapter 7 considers the research questions from the perspective of individual learning narratives. First, incidents of learning are identified in relation to the analysis of types of inter-organisational relationships. This develops the ideas already outlined in the previous chapters. Accounts relating to processes of individual learning are considered, particularly in cases where individual positions within organisations require learning. The implications for the inquiry of how IOL affects partnership in IOR are investigated in various cases. It also compares cultures of learning across the case study organisations.

Finally I conclude with the implications of the arguments and their contributions to the delivering of development aid. 


\section{CHAPTER 1 PARTNERSHIPS: MEANINGS, PRACTICES AND COMPLEXITIES}

\section{Introduction}

The first part of the chapter connects the questions related to the central issues of my research to a wider context. It concerns the ways in which previous writers have conceptualised partnerships and examined how they have emerged, developed and become popular practice in various arenas of social life. A partnership may refer to relations between individuals. In this research it refers to clusters of organisations working together towards some common purpose. For this reason, attention has focussed on research into the nature of the interaction between organisational partners, both at a general level and within the specific context of the aid sector. After defining the term "partnership", I review writing that considers partnership co-ordination. Firstly, the literature on the analysis of inter-organisational co-ordination is reviewed, in order to describe the parameters of partnership, their diversity and how their different characteristics relate to each other. The motives, procedures and mechanisms of partnerships are examined. These are then compared with those of interorganisational networks. This approach allows me to position the analysis of partnerships in various fields, including political economy and organisational and institutional studies. The chapter considers aspects of inter-organisational relationships that facilitate co-ordination, before examining experiences of partnerships in different fields of practice. This reveals the complexity of 
partnership management in terms of organisational structures and interorganisational relationships.

In the latter half of this chapter, I explore the popularity of partnerships as a contemporary trend in policies and strategies to achieve technical co-operation in the delivery of international development aid. Also the implications of the increasing importance of partnerships are considered with reference to recent development approaches. Attention will focus on hierarchical organisational relationships in the aid sector in order to ascertain how partnerships are perceived in theory and in practice. In conclusion, I identify issues emerging from my reading, of which I wish to take account, in pursuing this research.

\section{Partnerships in Social Life}

There are many definitions and forms of partnership. This section seeks to contextualise the popularity of the term and the ways in which it may be conceptualised. An exploration of how the practice of partnership has developed is then carried out.

Partnerships may involve both individual and institutional relationships, and may be either inter-personal, inter-professional or inter-organisational. They may, further, comprise either formal relations regulated by official contracts or informal interactions without official commitment. However, a central concept of partnerships is that they emerge on the basis of common values and interests, but may be arranged both organically and coercively. Despite the frequent use of 
the term, there are different practices and interpretations which depend on the basis of the relationships between partners. The term is often "an imprecise and inclusive concept capturing a wide range of behaviour, attitudes, values, practices, tools and techniques" (Bresnen and Marshall 2000: 231). The question arises as to whether there is a new connotation attached to the term today, or whether the term replaces those previously used with little significant change either in practice or discourse.

Dictionary definitions are useful starting points in an attempt to categorise the different types of partnership. The meaning of "partner" must be clarified before the relationship between partners can be defined. The following are the Oxford English Dictionary definitions:

A. Partner 1. One who has a share or part with another or others; one who is associated with another or others in the enjoyment or possession of anything; 2. One who is associated in any function, act, or course of action; one who takes part with another or others in doing something; an associate, colleague. 3. One who is associated with another or others in the carrying on of some business, the expenses, profits, and losses of which he proportionately shares. 4 . One who is on the side (of any one)... (OXD II, 1989: 512).

B. Partnership 1.The fact of condition of being a partner; association or participation. 2 An association of two or more persons for the carrying on of a business, of which they share the expenses, profit, and loss. 3.The rule or method for the calculation of a partner's share of gain or loss in proportion to his share of the capital or other determining conditions (OXD II, 1989; 512).

In this thesis, "partnership" refers only to inter-organisational relationships as stated in B 2 and B 3 above. I examine accounts of some of the features of interpersonal partnerships where these assist the understanding of the relationships 
between organisations. Although the dictionary definitions clarify the structure of inter-organisational relationship, the term connotes the different structures and the quality of relationships in different sectors of society.

The new popularity of partnerships may be observed in the commercial and statutory sectors, where they are expected to achieve specific objectives. This is demonstrated, for instance, in the establishment of the Institute for Public Policy Research, the UK's commission on Public and Private Partnerships (1999). This was driven by the need for a more robust account of the potential role of private and voluntary organisations within the public services (IPPR 2001). The increasing pattern of partnership is likely to be the same in the aid sector. With regard to the popularity of partnerships, the question arises as to what they are expected to achieve. The problems associated with previous relationships and possible changes need to be examined along with the motivations that lie behind the commitment of organisations to partnership.

\section{Emergence of partnerships}

The phenomenon of increased inter-organisational relationships may be attributed to the responses of organisations to change in their environmental conditions. Chisholm (1998) claims that with globalisation, technological change and knowledge have steadily increased. They have brought new complexity and turbulence to organisational environments. He describes the characteristics of shifting environments in which strong international competition and rapid and pervasive technological change, together with the growing importance of 
knowledge, cause an ever-increasing demand for quality and pressures of cost control. Similarly, a business management scholar, Ebers (1997), examines aspects of inter-organisational co-operation. He claims that the motives for which organisations engage in partnership relationships are related to the objectives of increasing their revenue and reducing costs and cost-related risk, although other motives are also involved.

A slightly different view of such motivations may be obtained from a consultant in environmental affairs. Davis (1999: 58) points out that not only companies, but also government and non-governmental organisations have experienced reengineering and a reallocation of resources. He observes the decision of those sectors to enter into partnerships as being drawn by a reduction in revenues and a shortage of time. Davis explains the functions of partnership as "uniquely designed to work best with highly profound and complex environmental problems and allow everyone to experience the "win-win" attitude...."(58). According to Davis, organisations engage with partnership arrangements to eliminate duplication of effort, and to increase equity in relation to specific issues. He refers to cases of non-traditional arrangements, such as when corporations work with NGOs. This kind of relationship, based on corporate good, is designed to provide a link to new markets and to establish measures of success. One major justification for this increased inter-organisational coordination may be found in organisational environmental changes resulting from the global market reforms of the last thirty years, which have led organisations to engage in shared financial strategies. Therefore, partnerships have emerged as 
tools to increase financial advantage. This enables labour and time to be cut, as required by the competitive pressure of the market, regardless of its demand for co-ordination.

\section{Co-ordination, networks and partnerships}

A deeper understanding of partnerships may be achieved by analysing the practices and functions of their co-ordination. Investigating this concept helps to understand how diverse actors in society find a way of relating to each other while at the same time protecting their individuality and working mechanisms. Studying co-ordination in social life from an economic perspective, Thompson et al., (1991) examine mechanisms of social life in terms of three models: markets, hierarchies and networks. At one level, the model of how these are co-ordinated (Thompson et al., 1991) calls to mind similar stories of partnerships. At another level, the detailed descriptions of characteristics and typologies of each institutional form help to conceptualise the diversity of scenarios of interorganisational relationships in various environments. Key aspects of these modes are dispersed competition, hierarchical control, and spontaneous solidarity (Streeck and Schmitter, 1991). For Thompson et al., co-ordination is

the bringing into a relationship otherwise disparate activities or events. Tasks and efforts can be made compatible by coordinating them. Bottlenecks and disjunctures can be eliminated, so coordination is usually discussed under a sign of efficiency (Thompson et al., 1991: 3).

This is seen as a positive aspect of co-ordination. Therefore these authors claim that various agencies can be 'ordered', 'balanced' or 'brought into equilibrium' 
by the act of coordination. Without co-ordination, these agencies might experience chaos and inefficiency.

The observations of Thompson et al. (1991) as regards co-ordination between markets, hierarchy and network reveal how actors with diverse functions substitute for each other in ensuring that the original functions of the mechanisms are maximised. Their suggestions regarding co-ordination emphasise a function of the market. Based on neo-classic economic thought such as that of Adam Smith (1776), pursuing self-interest in the market leads to the maximisation of the welfare, not only of individuals, but of society as a whole. This is achieved through the 'guiding hand' of the market with regard to what works through the exchange and price system. However, Thompson et al. (1991), go on to argue that such co-ordination cannot be achieved without a form of hierarchy. Administration as a means of control is essential to co-ordination. Nonetheless, the focus on both market and hierarchy neglects the informal mechanisms of networks, which are shaped by the cross-cutting chain of social, political and economic relationships.

Where diverse actors are involved in co-ordination, conflict and dispute may result. This is because the three concepts, markets, hierarchies and networks imply different understanding as to how people may be co-ordinated within their forms. Thompson et al. (1991: 4) argue as follows:

Each of these approaches represents a contested territory where different and often competing claims are made as to the proper understanding of their respective coordinative effects. 
One limit to co-ordination is the need for each actor to operate semiautonomously, which does not allow total co-ordination. This also depends on the fact that these different agencies deploy "competing conceptual apparatuses of calculation in establishing their objectives, sorting out their priorities, undertaking their activities"(ibid., 4-5).

The points made by Thompson et al. (1991) relating to co-ordination are useful parameters for understanding partnerships. For the sake of efficiency, all actors maximise their function and are sometimes forced to engage in acts of coordination. The problem of conflict between actors occurs because of the diversity of their approaches to co-ordination and of the need to maintain a certain degree of competition in order to preserve their autonomy and pursue their priorities.

Writing on international development, Harriss et al. (2000), in the introductory section of their book, suggest models which refer to the co-ordination of social life. Their generic models are defined in terms of competition, collaboration and co-operation. These concepts correspond to their distinctions between three institutional frameworks: markets; state and NGOs, trade unions and community groups. Harriss et al. (2000) explain that the motivation for organisations to choose co-operation as strategy is, in many cases, associated with the idea of participation, process management and organisational learning, as well as with the need to manage diverse interests. They comment that instead of leaving various interests in financially and politically co-ordinated action, co-operation 
as a concept allows diverse interests to be built into a whole new idea or approach. On the other hand, they criticise terms commonly associated with cooperation, such as partnership, since it "can in practice be little more than a smoke screen for many other kinds of relationship" (Harris et al., 2000: 8). They further argue that the power in co-operation, is based on imbalances of knowledge, expertise, and/or contribution.

From the perspective of the analysis of organisational linkages, the co-ordination of markets, hierarchies and networks occurs within hierarchical interorganisational relationships. The notion of network and co-operation, on the other hand, exists in horizontal inter-organisational relationships. In other words, co-ordination occurs as a direct result of the diversities of organisational hierarchy, whereas co-operation, as represented in a network, is usually seen in terms of rather flat relationships. Partnership arrangements in social life nowadays are practised in both situations.

Alternatively, a network might be regarded as a form of co-ordination in social life, which is in accordance with neither functions of markets and hierarchies, nor competition and co-ordination. A network can be referred to as a new form of organisation, replacing vertical communication and control relationships with lateral relationships (Hatch, 1997). Networks are characterised by "recurring exchange relationships among a limited number of organizations that retain residual control of their individual resources yet periodically jointly decide over their use" (Ebers, 1997: 4). Castells (1993) stresses the fact that the decreasing 
significance of hierarchy and the increase in horizontal relations are leading society to take on the characteristics of a network society. The formal ties binding the units of an organisation are replaced with "partnership" (Hatch, 1997). In business, "the idea of partnership sums up the central notion for a network" (Johnston and Lawrence quoted in Thompson et al.,: 14).

In the present research, the idea of linking organisations, which are neither hierarchically nor competitively associated with each other, as far as the concept of networks is concerned, is relevant to connotations of partnership, at least to those used at policy levels. However, this needs further exploration if it is to illuminate the mechanisms of partnerships. I argue that one of the main differences between networks and partnerships, in practice, is associated with the contractual nature of the relationship. Partnerships tend to be based on an explicit contractual relationship, while networks do not ${ }^{1}$.

Inter-organisational relations are inspired by similar motivations to those of networks. Organisations facing rapid technological change, shortened product life cycles, and fragmented, specialised markets, build networks (Hatch, 1997). Hatch emphasises the advantage of networks in terms of the financial efficiency resulting from a reduction in the administrative overheads of the traditional vertical hierarchy. By networking, organisations seek to control their environment by making links with each other to reduce their vulnerability to

\footnotetext{
${ }^{1}$ Some researchers visualise partnerships as organisational matrices, with actors positioned on vertical and horizontal relations. In this thesis, co-ordination implies hierarchical and dominant relations along the vertical axis. Co-operation indicates flatter relations on the horizontal axis. Both co-ordination and co-operation involve collaboration.
} 
environmental changes. This leads to the innumerable inter-organisational networks characteristic of modern societies (Morgan, 1990).

Nonetheless, networks based on friendship, reputation and shared ideology may have the capacity to generate co-operation and trust. Unless they have common values, based on simple economic relationships, the actors in a network may be exploited, either by their network partners or by suppliers (Hatch, 1997). Advantages of networks are that they can liberate decision-making, lead to the sharing of information and inspire innovation. However although the linkages of organisations would appear to be organically structured, such relationships are not automatic, and require management.

For some authors, networks are situated somewhere between economic rationality and authorised pressure (e.g. Thompson et al., 1991). Alongside market mechanisms, a network may emerge as an alternative route to financial efficiency. At the same time, the relationships of networking partners are not purely competitive, as with market-based ones, but there is a degree of control exercised by the hierarchical relationships within networks. In this respect, partnerships can be analysed in a similar way to networks, although account has to be taken of the specificity of partnerships. For example, whether the relationship is contractual or informal.

Within the hub of the network, organisations are connected by different interactions. This raises the question of how networked organisations share 
resources and outcomes gained from organisational linkages. Some aspects of networks, such as their informality and their horizontal relationships inhibit links between networking organisations. This is also related to the fact that the line between networking organisations may not be as clear-cut as is the case in market based or hierarchical relationships. In the context of this thesis, network analysis is particularly concerned with organisational relations in terms of their resources and values. As for linkages based on resources, resource dependency theory is particularly relevant to the aspects of networks in this study. Resource theory argues that organisations endowed with more resources control the power of other organisations within networks by trying to retain or reduce the access of others to the resources they need (Morgan, 1990: 193).

Many researchers emphasise inequalities within inter-organisational relationships. Benson (1975) claims that patterns of inter-organisational networking are dictated by political economy, and related to the struggle to secure the scarce resources of money, authority and legitimacy (Benson quoted in Dawson, 1986: 101). Morgan (1990: 186) states that the hidden agenda behind the analysis of networks is to understand the power relationships within them. Network analysis also offers representations of the channels through which resources, information, opportunities and influence flow (Hatch, 1997). Therefore, analysing networks can allow the complexities of structures of interorganisational relationships to be understood in terms of who dominates whom. 
Having recognised the importance of the analysis of networks for understanding inter-organisational relationships, this study will now focus on the implications of organisational interaction for the characteristics of individual organisations. Institutional studies analyse to what extent environments impact on organisational behaviour. DiMaggio and Powell (1991: 65) state that:

once disparate organizations in the same line of business are structured into an actual field (as we argue, by competition, the state, or the professions), powerful forces emerge that lead them to become more similar to one another. Organizations may change their goals or develop new practices, and new organizations enter the field. But in the long run, organizational actors making rational decisions construct around themselves an environment that constrains their ability to change further in later years.

DiMaggio and Powell (1991) use, as the basis for their discussion, the concept of the process of homogenization, isomorphism, which is defined as " a constraining process that forces one unit in a population to resemble other units that face the same set of environmental conditions" (Hawley 1968, quoted in DiMaggio and Powell, 1991: 66). Two types of isomorphism may be identified: competitive and institutional. Competitive isomorphism is related to free and open competition in the market. The institutional view of isomorphism, on the other hand, introduces forces that press communities towards accommodating to the outside world. The latter view leads to DiMaggio and Powell's view that " organizations compete not just for resources and customers, but for political power and institutional legitimacy, for social as well as economic fitness". Therefore, DiMaggio and Powell (1991: 66) use the concept of institutional isomorphism as a tool in the interpretation of modern organisational life which they believe is permeated by politics. They go on to relate the impact of 
interaction within organisations to individual organisational culture. Their analysis, which combines power and cultural aspects of the external social environment, is vital to understanding the processes and influences of partnerships.

Inter-organisational networks are often seen as being formed in a similar way to partnerships and as being driven by the same motives. Resourcé-based power relationships and dependency within networks have also to be taken into account, as have the effects of isomorphism. Inter-organisational relationships, such as those within partnerships, tend to be unable to lead to equal sharing of resources and autonomy, despite the idealistic connotations, which are often implied in the use of these concepts. Instead, organisations that are more powerful in terms of resources, status, or other dimensions, can disguise inequalities in implementation and co-ordination by hiding them behind the popular term.

\section{Partnerships and inter-organisational relationships}

In this section, various practices of partnership are explored, to provide insights into why and how organisations engage in such relationships. The diversity of structure and motivation of these organisational engagements suggests some implications for complexity in the implementation of partnerships. The following experiences are based on the available literature of the processes of building inter-organisational co-ordination and insight in diverse fields. They lead to the examination and investigation of the complexities of such organisational formations. 
There are several motivations for inter-organisational co-ordination. Some of them are led by financial efficiency, as observed, for instance, in inter-firm collaboration, while others stress factors such as mutual demand to substitute resources and exchange expertise in, for example, technology and knowledge. For instance, the recent increase in research and development of inter-firm collaboration is driven largely by cost-economizing, strategic and flexibility rationales (Hagedoorn, 2001). Other fields of practice also show motivation to be the result of technical gaps requiring input from other experts. For example, in some cases, partnerships develop between public and private sectors, when the state is unable to deal with problems and believes that the market will help to overcome them (Obirih-Opareh and Post, 2002). Other motives include the need for professionals to share technical knowledge, such as in the health sector (e.g. Makaram, 1995). Inter-professional co-operation provides a link between research-based knowledge and the practical talent of clinicians.

Whatever the motives, research into the processes and implementation of such diverse forms of organisational co-ordination reveal some difficulties. Several studies point out that it is not always the technical or financial aspect of partnering which is the principal factor behind difficulties in co-ordination. Difficulties often arise as a result of approaches to and management of coordination. Co-ordination between governments and communities often shows that there are constraints in existing approaches to partnership. One study suggests that partnerships between public authorities and various community 
stakeholders may be described as command-and-control approaches (Kironde and Yhdego, 1997: 213). Problems experienced in co-ordination are sometimes linked to corruption, poor relations between politicians and the general population, the politics of privatisation, and political apathy, rather than the issue of limited resources (ibid.). Kironde and Yhdego's study in low income Tanzania highlights inappropriate management as being a significant limitation to co-ordination, driven by asymmetric power relations between public authorities and communities. Public policies are also criticised because they exploit partnerships as institutional mechanisms to implement hidden political intentions, such as neo liberal objectives (Raco, 2000). Furthermore, in spite of encouraging bottom-up and community empowerment, partnership policies often result in local competition, where bids for government funding programmes are concerned (ibid.).

The ability to manage the uncertainties of the environment is also indicated as a significant element in organisational co-ordination. For instance, a study of the development of cooperative partnerships in electronic communication networks (Bytheway and Dhillon, 1996) suggests that management systems that can handle environmental turbulence to promote an understanding of organisational boundaries, and of the interdependence of firms. In this context, technological trends, competition, economic factors, and socio-political factors have a direct effect on developing appropriate partnerships in emergent, electronically networked environments. 
Many studies stress the importance of trust between partners. An example can be found in the work of Bresnen and Marshall (2000). They suggest that behind the presumed link between partnering and cultural change, at both organisational and inter-organisational levels of analysis, lies a real tension between the need for development of trust between clients and contractors and "surrounding economic conditions that predispose contractual partners to act in more 'traditional', adversarial and even exploitative ways" (Bresnen and Marshall, 2000: 233). They claim that complexities of organisation such as a management style which effects change, behavioural manifestations and vertical and horizontal differentiation as concepts of organisational culture should be appreciated, in order for a more realistic and practical approach to the development and implementation of partnering to emerge. Where partnership is associated with changes in an organisation's understanding of aspects of organisational mechanisms and culture, this may be crucial to the analysis of how partnership works (e.g. Bresnen and Marshall, 2000).

Hence, the achievement of partnership has different meanings, depending on the relations within individual organisations. Some of the above research suggests that better resourced and higher status organisations try to control partners. They attempt to exploit co-ordination for their own benefits, and to use partnership policies as instrumental strategies. A number of practices suggest that environmental uncertainty and organisational change are significant in the management of partner co-ordination. Several studies emphasise that building relationships based on trust, not merely on the basis of contractual economic 
links, provide a different set of circumstances from the traditional competitive relationships.

So far, this discussion has presented general ideas about contemporary interorganisational partnerships, as well as reflections on how organisations influence their development and inhibit their growth. The aim, now, is to consider the implications of these conceptualisations for partnership in the aid sector.

\section{Partnerships and Aid}

In this section, partnerships in the aid sector are examined in the light of various organisational relationships. Organisational actors involved in the aid system are identified in order to provide an overview of the roles they play. Following this, the emergence of partnership policy as a recent approach to delivering aid is considered, with particular reference to the co-ordination of relationships between government and non-government organisations.

\section{Aid overview: actors and approaches}

The various stakeholders involved in the international aid system are described. An overview of the aid system is provided which identifies actors in different types of organisation. Also the relationships between donors and recipients in the aid system are presented. 
The objectives of foreign aid are to promote long-term growth and poverty reduction in low-income states, and to promote the short-term political and strategic interests of donors (World Bank, 1998). Aid constitutes a transfer of resources in the form of either capital assistance or technical assistance. The former is investment finance comprising loans and grants and the latter comprises experts, training and technology (Youngman, 2000). Foreign aid is usually associated with official development assistance (ODA) and is normally targeted at poor countries. ODA is a subset of official development finance, and comprises grants plus concessional loans that have at least a 25 percent grant component. Official Development Finance (ODF) flows between high-income country governments, multilateral agencies and low-income states (World Bank, 1998: 6). Both types of aid, ODA and ODF can be divided into multilateral and bilateral components. Multilateral assistance is funded by contributions from richer countries and administered by appointed agencies, whereas bilateral assistance is administrated by agencies of donor governments. Some bilateral aid is tied. That is, it must be used to procure goods and services from the donor country (ibid.). Apart from these agencies, the non-governmental actors involved in international development assistance include private, not-for-profit and voluntary organisations. Then there are the multilateral financial institutions, which have probably the most significant influence on aid policies and strategies, particularly in relation to economic development.

In order to explain the high degree of influence exercised by multilateral organisations, we need to refer back to the aftermath of World War Two, when 
two initiatives were established to reconstruct the world economy: the Marshall Plan and the Bretton Woods programmes. The purpose of the Marshall Plan was to facilitate bilateral initiatives between two states, whereas the Bretton Woods programme was a multilateral agreement involving several member states (Hewitt, 1992). The idea of the latter was to solve the problem of capital scarcity and declining terms of trade. This involved moving from nation-centred economic behaviour to internationally co-ordinated finance and trade within a new world system of large-scale international income transfers (Hewitt, 1992: 223). The Marshall Plan implied bilateral transfer for reconstruction from the USA to European states and Japan. The four Bretton Woods institutions are the International Bank for Reconstruction and Development (IBRD), later known as the World Bank; the International Monetary Fund (IMF); the United Nations (UN) and the General Agreement on Trade and Tariffs (GATT) ${ }^{2}$. The World Bank provides long-term finance for investment, whereas the IMF is a source of short-term finance to compensate for balance of payments deficits and exchange rate fluctuation. The $\mathrm{UN}$ acts as the forum through which international decisions are taken, and is the means by which international political and military stability is maintained. The role of GATT (now, the World Trade Organisation) is to regulate international trade and stabilise world commodity prices. Through these institutions, the financial and economic policies of the world have been regulated, disproportionately favouring high-income states. This is attributable to the inequality in votes and nationalities that initiate leadership, such as the US for

\footnotetext{
2 The UN established 1945 has today 189 member countries. It has six main organs: General Assembly, Security Council, Economic and Social Council, Trusteeship Council and Secretariat. Specialised agencies such as the World Bank and IMF are independent autonomous organisations working with the UN and each other through the coordinating machinery of the Economic and Social Council (UN). There are a multitude of their UN agencies.
} 
the World Bank and the Europeans for the IMF (Hewitt, 1992; McMichael, 2000). In 1961, the dominant countries formed the Organization for Economic Co-operation and Development $(\mathrm{OECD})^{3}$. The Bretton Woods agreements remained intact as an international system until 1973, and even now, the four institutions have considerable influence on low-income states (Hewitt 1992).

During the 1980s, the imposition of Structural Adjustment Prográmmes (SAPs) by the IMF and the World Bank, on low-income states brought dramatic economic change ${ }^{4}$. By the mid-1980s, SAPs were fully established as a recipe to overcome economic crisis and they created macro-economic conditions for support. They became the rule within the international donor community (Sifuna, 2000: 6). Under the conditions of loans from the World Bank and the IMF, the SAPs were designed to reduce the role of national government in economic and social development. This was achieved by measures such as privatisation and cuts in public spending. The SAPs required a significant cut in the budget of the social development sectors, such as health and education, in low-income states. They contributed to making the poor even poorer (ibid.).

\footnotetext{
${ }^{3}$ The OECD groups 30 member countries sharing a commitment to democratic government and the market economy. With active relationships with some 70 other countries, NGOs and civil society, it has a global reach. Best known for its publications and its statistics, its work covers economic and social issues from macroeconomics, to trade, education, development, science and innovation. OECD work on development is carried out primarily by the Development Cooperation Directorate/DAC, the Development Centre, the Sahel \& West Africa Club and the Centre for Co-operation with Non-Members. They work towards contributing to the economic development of both Member and non-Member countries (OECD).

${ }^{4}$ Structural adjustment loans were designed by the World Bank to foster economic reform in the less developed countries by supporting measures to remove excessive governmental controls, getting factor and product prices to reflect scarcity values, and promote market competition (Todaro 1997: 721).
} 
Bilateral governmental organisations rely on the client government establishment of a free market to further their foreign affairs interests. Most of the member countries of the OECD have a ministry or equivalent department responsible for overseas development. Examples of these are the UK Department for International Development (DFD); the United States Agency for International Development (USAID); the Canadian International Development Agency (CIDA); the Japan International Cooperation Agency (JICA) and the Swedish International Development Cooperation Agency (SIDA). Foreign aid is often positioned within departments of foreign affairs. It has to follow their international relations policies, as well as general national development policies.

Figure 1 shows the major organisational actors who are involved in aid delivery. The arrow indicates a flow of finance to recipient countries through these organisations. The curved line separates international and national giving and receiving organisations, the flow of finance and intervention take place through cross-national interactions.

ODA to low-income states experienced an upward trend in the 1960 s, reaching a peak in 1992. However the volume of aid has decreased. In 1997, aid dropped by 23.7 percent compared with the 1992 figures (Hjertholm and White, 2000: 85). There were three reasons for the reduced importance of aid: fiscal problems in OECD countries; the end of the Cold War and significant growth in private capital flow to low-income states (World Bank, 1998). 
Figure 1 Major actors in aid delivery

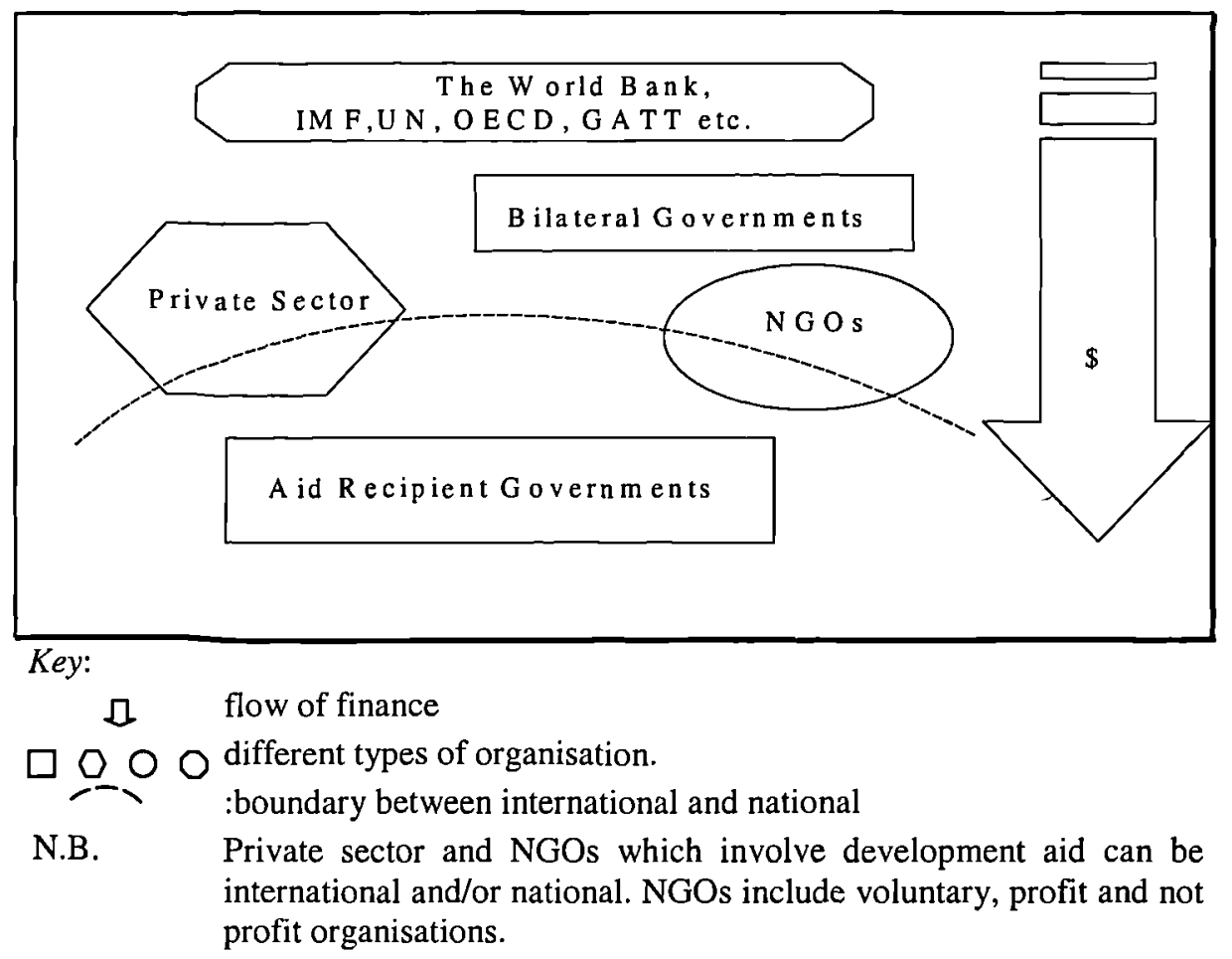

\section{Partnerships in the aid community}

The use of the word partnership has grown significantly in the aid sector, where formally various organisations, differing in terms of institutional culture and capacity, were involved in pursuing their own policies, objectives and strategies, separately and in collaboration with others. According to Fowler (1997), since the 1970 s, the notion of partnership has been used to express an ideological aspiration of international solidarity in the area of development, and covers many different kinds of relationship between varying development agencies. In the last few decades, the demand for actors within the public, private and voluntary sectors has dramatically increased. This phenomenon has brought about a 
number of changes, both in the relations between these actors and in the ways in which they operate individually.

The political and economic transformation of the world has determined trends in foreign aid in recent decades. For Fowler (1998), the priority of international aid today is market competition, and the maintenance of political stability and economic growth within countries in low-income states. In the 1990 s, there was an increase in public demand for information about the use of aid in terms of efficiency, in addition to the incidence of domestic privatisation policies which resulted in aid being allocated on contractual terms. This led to the non-public sector constituted by private and non-profit actors, becoming involved in development aid practices (Fowler, 1998).

Similarly, Robinson (1993) claims that since the end of the Cold War in 1987, bilateral and multilateral donors have dominated development policy by imposing a 'New Policy Agenda', based on neo-liberal economic and political theories. In the context of the new policy agenda, the market and the private sector are perceived as conducive to economic development and the provision of services. Meanwhile, the role of the state in the economy is kept to a minimum, and NGOs are now seen " as the preferred channel for service-provision in deliberate substitution for the state" by official agencies (Hulme and Edwards, 1997:6). Commins (1997:14) points to:

The donor-driven New Policy Agenda emphasises the central importance of free markets, efficient use of limited government resources, a reduced role for the state and the need for good governance in low income countries. 
Therefore, the shift from state directed development to market driven development has been accompanied by increased amounts of bilateral and multilateral funds being channelled through NGOs. This has meant that NGOs have come to take responsibility for what and where previously state managed programmes, serving as implementers of public sector donor priorities (Commins, 1997). For donors, the pressure to limit the use of public funds grew, as they were required to scale down programmes due to changing domestic priorities and burgeoning deficits. In consequence, the demand to demonstrate the real benefits of aid programmes increased (Ross, 1997:46).

Under the New Policy Agenda, NGOs are perceived as essential components of the civil society and as a means of encouraging democracy ${ }^{5}$ (Hulme and Edwards, 1997). Neo liberalisation and democratisation have brought about a shift in the number of NGOs as actors in the economic and political development process. This has resulted in an increase in the share of NGOs funded by public money. Therefore the public has increasingly perceived NGOs as partners in development.

\section{The funding of NGOs}

The growth in the importance of development NGOs was observed in the mid 1980s (Hjertholm and White, 2000). NGOs in the industrialised states have grown from 1600 in 1980 to 2970 in 1993 (Smillie and Helmich, 1993). A

\footnotetext{
${ }^{5}$ NGOs are one of the major organisations in civil society. Civil society is organised by voluntary initiatives through identification of common goals, values and needs (Harriss et al., 2000) which character is different from organisations in either the state or the market, as it is neither depends on the coercive power of the state nor self-interest where success is determined by measuring rod
} 
similar scenario, in terms of growth, applied to NGOs in the low-income states (Hulme and Edwards, 1997). OECD member states increased their aid to NGOs from 0.7 per cent of the total in 1975 to 3.6 per cent in 1985 and 5.0 percent between 1993 and 1994. The World Bank estimates that the total income of NGOs derived from donors increased from 1.5 percent in the early 1970 s to about 30 percent in the mid-1990s (Hulme and Edwards, 1997).

The large international NGOs (INGOs) of today started as a direct result of wars and emergencies. Until the-mid 1970s, they designed, funded and managed their own activities in poor countries, with little contact with local NGOs (LNGOs), of which there were few. One of the reasons for this was the belief based on " a we know best " attitude (Simillie and Hailey, 2001: 33). However, during the early 1970s, INGOs started to realise that LNGOs could reach the poor more quickly and began to work with them and finance their activities. They were also cheaper than expatriates' expertise. By the mid-1980s, bilateral agencies began to add their support to LNGOs, either directly or indirectly, through INGOs. Multi-lateral agencies became involved the NGO scenario in the 1990s, either with grants or through contractual arrangements (ibid). Recently, the private sector has started to link with NGOs.

The increase in the amount of international funds to NGOs is affecting the relationship between donors and NGOs in various ways. In a special issue of the international journal World Development, about NGOs, Garilao (1987: 113)

of money (Brett, 2000). 
raised the question of whose development agenda was being pursued, and whether, in fact, such resource transfers resulted in a new form of dependency. In the late $1980 \mathrm{~s}$, state-NGO relationships were cautiously recognised as a result of the dramatic increase in public funds given to NGOs in the previous decade. A number of researchers have warned, however, that the increased dependency of NGOs on public funds implied a loss of identity and autonomy (e.g. Van der Heijden, 1987; Garilao, 1987). For Van der Heijden (1987: 111), funding was developed associated with the promise of more adequately reconciling the requirements of NGO autonomy, program integrity and operational effectiveness as well as public accountability. Questions continued to be asked in the following years. In 1992, Edwards and Hulme (1992) stated that one of the challenges for NGOs in the new millennium will be how to solve the dilemma of increasing their developmental impact without losing their traditional flexibility, their value-based nature and their effectiveness at the local level. In other words, NGOs are faced with the dilemma of relying on donors' financial contributions, while at the same time, escaping the very danger of the isomorphism previously discussed. Edwards and Hulme (1992) analyse how significantly national and international policy processes of macro level intervention determine the extent to which NGOs achieve their objectives and develop their impact.

Some risk resulting from increasing donors' direct funding of LNGOs is now recognised. Bebbington and Riddell (1997) argue that for donors, increasing direct funds to LNGOs can be cheaper than channelling them through INGOs. The donors' objective is also to deliver aid within the context of their own 
bilateral aid programme. Hence, increasing funds to NGOs does not coincide with the donors' claim that they are strengthening LNGOs as representative civic organisations in client states.

There has been a growing development of interest in the collaboration between states, private organisations and NGOs. The interactions of these actors are observed as one of the strategies for poverty reduction in the development process (Clark 1995). Clark continues to point to a transformation of NGO roles as they become important agents in society. This reflects the passage from a "supply side" approach to development based on delivering services or development projects, to a "demand side" emphasis, that focuses on helping communities articulate their preferences and concerns in order to be active in the development process. Clark also resists the idea of perceiving NGOs as development alternatives, but emphasises that NGOs' activities become significant only when they influence national development, and that state-NGO relationships should be complementary rather than competitive. In such processes, NGOs are required to develop partnership skills that enable them to act as agents between communities and official bodies. However, in spite of a growing understanding of the benefits of collaboration between states and NGOs, there are tensions and frictions between them. These are caused by differences in values, ideology, development priorities and approaches (Garilao, 1987). Ideal relationships are based on mutual respect, acceptance of autonomy, independence and pluralism of NGO opinions and positions. Nevertheless, such 
relations are rarely achieved, even when the conditions are favourable, because mutual distrust and jealousy seems to be deeply rooted (Fowler, 1992).

Hence, the increasing demand to work with other actors is becoming a necessity, in order to achieve internationally set goals for eradicating poverty. It is also driven by increased global competition, which has brought a reduction of public expenditure on international aid in many governments involved in bilateral programmes. It is thus necessary for organisational survival. As a result, partnership has emerged as a strategic approach, both in order to increase access to project/programme funding through collaboration with other specialised actors, and to survive, given the realities of tightened budgets. Players in this partnership game in the aid sector include multilateral and bilateral organisations, international and local NGOs, national and local governments, private services providers and community based organisations (CBOs)

Referring back to the model of co-ordination in the first part of this chapter, actors in the aid system relate to different types of organisation in order to fulfil their objectives. One of the key characteristics of co-ordination in the aid sector is that organisational relationships are hierarchically structured. Figure 2 describes this scenario. Located at the top of the hierarchy, the international financial institutions are most powerful in terms of their control of development aid finance and policies. They are followed by the bilateral aid agencies. A small number of international Foundations also make a significant financial contribution. Host governments that receive aid funds come after them. Aid 
implementing organisations, such as international and local NGOs, may come above or below the host government, depending on the funding system: that is, whether funding goes through the government or is directly transferred to local counterparts. Community based organisations (CBOs) and other groups which implement the funded projects are at the bottom of the hierarchy together with benefit users at grass roots level. Within the entire hierarchical organisational structure, networks of people emerge across organisational boúndaries either horizontally or vertically. It is assumed that individuals potentially act beyond the restrictions of organisational hierarchical relations.

Figure 2 The aid system hierarchy and networks

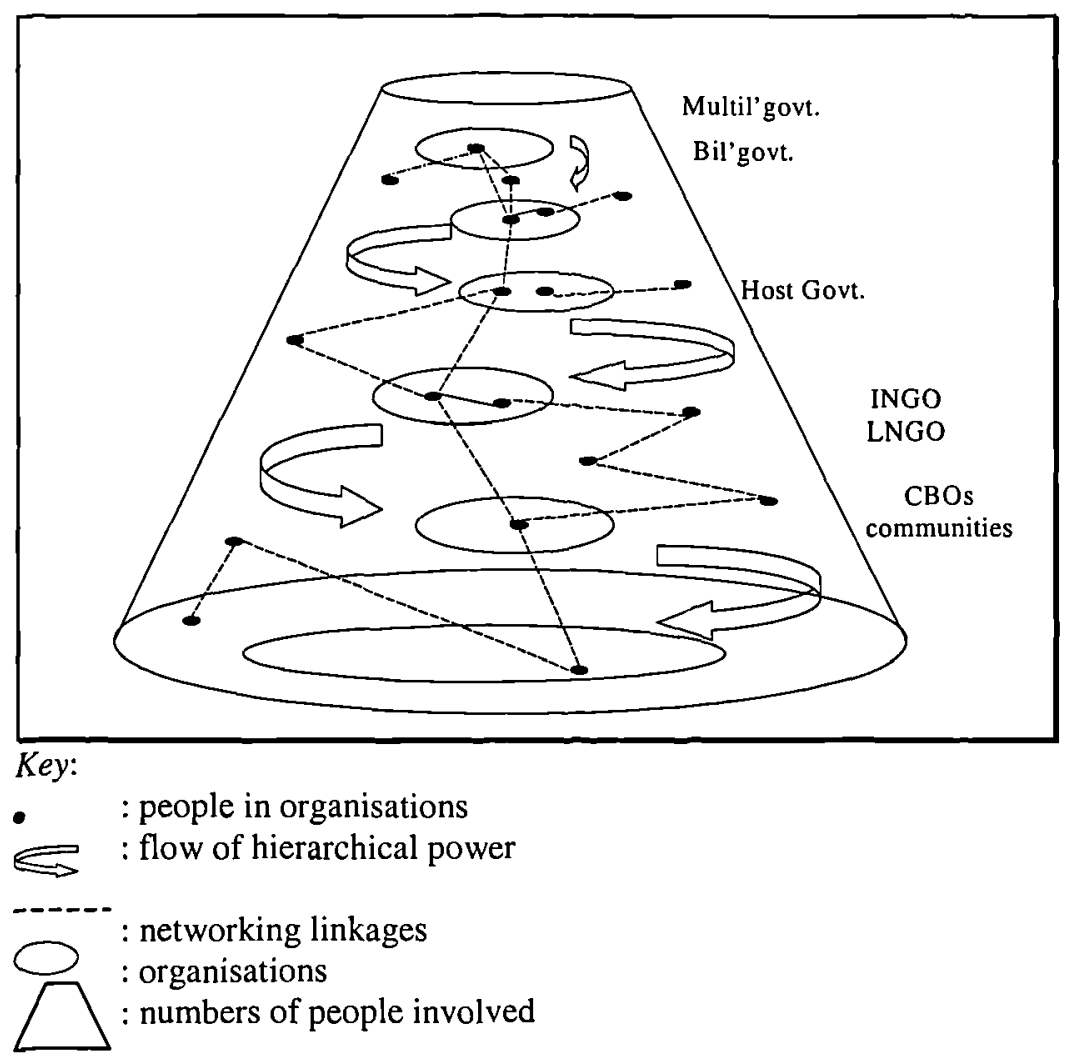




\section{Recent approaches to partnership}

International Financial Institutions have been criticised for the failures of the SAPs to reduce poverty. The World Bank and IMF have moved from the topdown approach of SAPs to a more participative process that brings local stakeholders together to help define national, social and economic policies for poverty reduction (Fukuda-Parr et al., 2002: 5) ${ }^{6}$. In the 1990 s, a new series of approaches to development was introduced by the Bank and $\mathrm{IMF}$, in collaboration with recipient governments. They introduced the new development strategy of the Development Assistance Committee (DAC) of OECD; sector wide reforms; a comprehensive development framework $(\mathrm{CDF})^{7}$ and Poverty Reduction Strategy Papers (PRSP) ${ }^{8}$. Key concepts leading these strategies are "ownership" and " partnership" (World Bank, 2002d). Nevertheless the practice of ownership and partnership through renewed strategies is continuously questioned (Buchert, 2002; Sifuna, 2000).

\footnotetext{
6 "In September 1999, the World Bank Group and the IMF determined that nationally-owned participatory poverty reduction strategies should provide the basis of all their concessional lending and for debt relief under the enhanced Heavily Indebted Poor Countries (HIPC) Initiative. This approach, building on the principles of the Comprehensive Development Framework, has led to the development of Poverty Reduction Strategy Papers (PRSPs) by country authorities for submission to the Bank and Fund Boards" (World Bank 2002a). Heavily Indebted Poor Countries (HIPC) Initiative is an agreement among official creditors to help the most heavily indebted countries to obtain debt relief.

7 "The Comprehensive Development Framework is an approach by which countries can achieve more effective poverty reduction. It emphasizes the interdependence of all elements of development - social, structural, human, governance, environmental, economic, and financial. It advocates: a holistic long-term strategy; the country in the lead, both "owning" and directing the development agenda, with the Bank and other partners each defining their support in their respective business plans; stronger partnerships among governments, donors, civil society, the private sector, and other development stakeholders in implementing the country strategy; and a transparent focus on development results to ensure better practical success in reducing poverty (World Bank 2000b)

8 “ Poverty Reduction Strategy Papers (PRSP) describe a country's macroeconomic, structural and social policies and programs to promote growth and reduce poverty, as well as associated external financing needs. PRSPs are prepared by governments through a participatory process involving civil society and development partners, including the World Bank and the International Monetary Fund (IMF)" (World Bank 2000c).
} 
The issues of partnership and ownership have been emphasised in the international development aid community, both in terms of aid providers preparing for aid proposals and aid recipients' rights against the conditionalities and dependencies of the 1990s. A new assistance approach with an emphasis on partnership and local ownership has developed to overcome the aid fatigue of traditional aid providers, in the context of increased need for international aid. Programme assistance and sector-wide approaches, or sector investment programmes, refer to a new assistance modality, which is assumed to increase the efficiency and effectiveness of aid (Buchert, 2002). Regardless of the multigovernmental statement on partnership in development cooperation, what happens in practice appears to be far from ideal. Considering the role of NGOs in these partnership programmes, Fowler (1998) claims that there are few NGO relationships that have the qualities of true partnership. Smillie (1997) suggests that development stakeholders should change their mind-set in order to contribute genuinely to developing NGO capacity in terms of management and finance. Since NGO capacity development can impact on the processes of interorganisational relationships (IORs), there seems to be a suggestion here for donors, state, and NGOs to learn from each other and change so as to maximise their collaboration.

\section{Critiques of development partnership}

In the arena of international development, the types of organisational actors involved may be more diverse than in purely profit-making areas. This is the result of pluralities in culture, sectors, geographical locations and objectives and 
aims of organisations. Variety can also be found in the capacity (resource, finance, and human) and status of organisations (social, political and religious). Above all, foreign aid, as a strategy of political economy, adds complexity to the plural elements of inter-organisational relationships. Therefore, the analysis of partnership in such settings requires an understanding of the determinants of the structure of relations between organisations.

Throughout the implementation of projects and programmes, inequalities in relationships can be observed in terms of access to resources, voice in decisionmaking processes, and outcomes obtained. According to Fowler (1998: 141), it was initially supposed that partnership would mean working together with equal recognition and mutual respect for identity, position and role. In practice, no one can deny the inequalities between partner organisations. The question is whether the concept of partnership used by policy makers can be realised, or whether partnership is merely a slogan to camouflage the highly skewed structures of new modalities of state and NGOs relationships.

Fowler (1997) criticises partnership as an "over-loaded and abused concept in the development lexicon" followed by 'participation' and 'empowerment' (272). Preston and Preston (2001), meanwhile, see partnership as "a guiding metaphor" for international development co-operation at the end of the millennium, albeit with a blurred definition. They remark as:

Whatever its connotations, there are no assumptions about equality of status, access to resources or contributions to be made by organisations within the networks that are created by different kinds of partnership between members (Preston and Preston, 2001: 3). 
Under these circumstances, the time has come to examine some of the research that is being undertaken on IORs in the aid sector, and how it relates to a different conceptualisation of partnership.

\section{Studies of inter-organisational relationships in aid partnerships}

This section starts by analysing hierarchical structure in the aid sector in terms of financial and ideological aspects. Stone (2001) examines the World Bank and the recently established Global Development Network (GDN) in the light of global public policy networks and knowledge networks. More recently, Buchert (2002) has conducted research into new multilateral and bilateral forms of partnerships and sector wide approaches (SWAPs) with a comparison of three African countries. Wallace (1997) analyse inter-organisational relations between UK NGOs and their donors. Preston and McCaffery (2002) examine partnership processes and consultancy-related communication issues in complex projects implemented in six countries and three continents.

Stone (2000) observes the specific form of identical power as being central to emerging patterns of global governance. The World Bank has focused its recent policy on becoming a "knowledge bank". The Global Development Network (GDN) is one programme in its operations. She argues that although GDN might lead to knowledge creation and sharing which would be beneficial to the public, the process has 'club-like' characteristics. The connection with the World Bank limits the GDN from being a knowledge seeking network which can become 
embedded in a range of global public policy networks, thereby weakening its multi-disciplinarity. Hence, GDN appears to be characterised by an unequal distribution of network resources and positions. Her study implies a gap between the rhetoric and the reality of the globally structured framework in the respect of information sharing due to unbalanced organisational relationships within the network.

Buchert (2002) compares three African countries, Burkina Faso, Ghana and Mozambique, in terms of the concepts and applications of SWAPs and partnerships. The study is based on interview data from government representatives and members of key agencies in the education sector. The study highlights structural constraints in the ways in which governments and aid agencies relate to each other in SWAPs and which do not allow partnership to develop beyond a rhetorical level. She argues that the process of implementing SWAPs, so far, has shown the difficulties of structural constraints, such as the settings of the approach, the institutional and human capacity to implement it and the differences in the interests of all the actors involved. Buchert further points to the SWAPs as being set within a macro economic and political framework, to the exclusion of educational experts for educational decisions. This approach enhances negotiations and decision-making at the top of the government system with experts in macro economy and finance. Her study shows that this new donor approach, based on the lessons of past experience, does not resolve the issue of what is actually needed by host governments. Buchert suggests that:

The level of rhetoric concerning mutual respect, transparency, trust, dialogue, cooperation, coordination and genuine partnerships cannot 
eliminate underlying differences and structural relationships between aid providers and aid recipients. Greater transparency of underlying interest and stipulation of the boundaries for mutual interactions might, on the other hand, contribute to developing the trust and respect which are needed for the further dialogues, co-operation and coordination of all development partners (Buchert, 2002: 83).

Research carried out by Wallace (1997) on UK NGOs within the changing funding environment examines donor-implementer relations at the level of practice. Their methodology is based on interviews with staff from seventeen UK NGOs and three key donors. The study analyses three managerial tools: strategic planning; logical framework and evaluation with questions of why and for what they have been used. Evidence shows that using the case of such tools is culturally mediated, and thus far from neutral, and is imposed by donors for their convenience. They claim that these tools emerge from the view of the new right approach being driven by the market and based on a corporate culture. The use of these tools therefore contradicts the nature of development and the peopleoriented approach of NGOs. Their research accentuates the inappropriate nature of the management of funding by donors in supporting the work of NGOs as a part of aid system scenarios.

Buchert (2002), meanwhile, examines partnership at a macro level with the case of SWAP, while Wallace et al (1995) analyses the case between UK NGOs and their donors. Preston and McCaffery's (2002) analysis of complex projects investigates partnership issues in their entirety, both at a policy and implementation level. They examine partnership processes and consultancyrelated communication issues in projects in six countries set within South and Central Asia, Africa and Eastern Europe. Interviews were held with members of 
communities, project implementing and management organisations, representatives of bi-lateral funding organisations, International Financial Institutions and consultants. The study examines the impact of global agenda on each project's management processes and outcomes.

As far as organisational cultural influence is concerned, there seems to be an international unity forcing the agenda from the top to the bottom of the hierarchy in international development agencies. Thomas (2000) claims that the concept of 'development' has been limited to liberal capitalism as the dominant mode of organisation, and he argues that only some 'developers' have sufficient power for their interpretation of 'development' to be imposed effectively on others. The most powerful 'developers', such as multilateral financial agencies, state agencies and their allies tend to refer to development in terms of alleviating problems.

In terms of the present thesis, an essential aspect of partnership may be seen to be the degree of participation in decision-making and in access to resources, both in general and specifically in the aid sector. In addition, the importance of individual capacities, such as the role of senior managers, and personal friendships and networks should not be ignored in seeking to understand partnerships. Several practices indicate that learning from partners, as well as learning about oneself, has the potential to impact positively on the processes of partnerships. Researchers have pointed out that differences in organisational 
structure, national culture, systems, and values are important in determining the processes of partnership.

Stone (2000) points out that knowledge sharing in GDN occurs as a result of a club-like network dominated by the World Bank. Buchert (2002), meanwhile, claims that the concepts and practices of partnerships observed in SWAPs remain at the rhetorical level. They are not contextualised by sector or country, as the approaches are managed at the macro level by finance officers. She argues that SWAPs should not be considered as a blueprint, but rather, as an "evolving process that depends on flexible and adaptable learning by all involved parties" (82). The above studies reveal some of the limitations of partnerships. However, as Buchert stresses, the importance of learning in the process of delivering projects and programmes requires a closer examination of the issue of learning, its nature and development.

The wealth of research on partnership confirms its contemporary management importance across public, private and voluntary sectors. It reveals a considerable preoccupation with the ways in which organisational diversity affects partner relations. However, very little research is as yet being done on how the relations between partner organisations leads to learning between them. This is quite surprising, given contemporary management perceptions that organisational learning is an essential feature of organisational effectiveness. To the best of my knowledge, there is no research that explores partnerships and interorganisational learning in the context of the aid sector. 
There is a case for detailed analysis of learning and its place in partnerships, between the various levels of partner organisations. Such a study should aim to investigate the project process holistically, from policy to implementation. This will provide insights on the integrating effect of learning in both internal and external organisations. My research takes a step in this direction. Its aim is to understand how learning between aid sector organisations can influence interorganisational relationships, and the effect of these on project processes and outcomes. To achieve this aim, I shall now link available conceptualisations of learning in organisational contexts to those already discussed in relation to the study of organisational culture and network analysis. 


\section{CHAPTER 2 LEARNING AND ORGANISATIONS FOR PARTNERSHIPS}

\section{Introduction}

Having explored the meaning and practice of partnership, this chapter will examine how learning has been found to occur within and between organisations in general and then in respect of aid sector organisations ${ }^{9}$.

Two broad levels of learning may be recognised. One is the learning, to deliver specified services. The other is the learning, thought to be the main ingredient of human development projects that seeks to change community behaviour and attitudes. As a study of development processes, this research is more concerned with learning within and between stakeholder organisations working together in partnership, than with the community level basic learning, intended as an outcome of human development intervention.

The chapter begins by presenting an account of the contemporary importance of learning in organisational and inter-organisational contexts, in general and in the aid sector. This paves the way for a more detailed review of research theorising the learning process and conceptualising its place in IORs, in increasingly common partnership arrangements.

\footnotetext{
${ }^{9}$ The learning processes of the benefit users of the selected projects for this study are also examined.
} 
Decades of development practice suggest a noticeable lack of learning from the experiences of people and organisations who are part of the aid policy and implementation process. In contrast, in the business sector, the ability of an organisation to learn faster than its competitors is claimed for a long time as a key strategy for success. In the knowledge economy, learning between organisations in partnership relationships across sectors is becoming a required skill. This is particularly, the case in profit-making sectors, where competition drives financial efficiency and rapid change. Inter-organisational partnerships in the business sector, often formed as joint ventures and strategic alliances, recognise the connection between learning and their commercial activities. Although alliances vary with regard to their defined purpose, time frame and structure, such as the joint research and development of a marketing arrangement, the overall nature of alliances provides a meaningful context for inter-organisational learning (Levinson and Asahi, 1995; Pollard 2001). A number of authors stress learning as an important ingredient of success in business alliances (Levinson and Asahi, 1995). They also recognise that knowledge of how to manage learning processes and information sharing plays a crucial role in determining whether alliances bring success or failure (Levinson and Asahi, 1995; Larsson et al., 1998).

Inter-organisational learning (IOL) in the aid sector has not been developed as much as in the business sector, nor has it been explicitly discussed in the literature. This is in spite of the fact that all kinds of IORs such as contracting- 
out, or even casual relationships, collaboration and networking, offer continuous opportunities for learning between partner organisations, which would enhance the quality of aid agency and implementers' activities and develop good relationships between them.

Interest in knowledge and learning in the aid sector has grown during the last few years. The World Bank has been driving its partners to become learning organisations and knowledge agencies since the late 1990s (Barnard, 2002). This trend is reflected in the OECD/DAC report (2001) Evaluation feedback for effective learning and accountability based on a workshop held in Tokyo in September 2000. Scholars are also examining the policies and practice of knowledge management, comparing their development across multi- and bilateral agencies (King and McGrath, 2002; King, 2002; McGrath 2002). Forss et al (1998) have concentrated more narrowly single organisational learning in bilateral agency. In contrast, Carlsson and Wolfgemuth (2000) have focused on individual experiences of learning in the aid organisations.

Despite the fact that the focus on learning and knowledge is relatively new in the context of aid policy, the concept of learning itself is implicitly used, in different forms, in the large and small-scale aspects of both daily and long-term task management practices. For instance, evaluation reports often include sections on "lessons learned" in the hope that organisations will avoid repeating their mistakes. A critical question is why this learning has not been explicitly 
recognised as an important factor in aid sector effectiveness and as a means of enhancing the quality of inter-organisational relationships.

One difficulty is that organisational relationships and inter-organisational learning in the context of aid partnerships have to be understood through the filter of individual learning. I am interested in investigating incidents in which individuals encounter opportunities for learning from others, particularly people belonging to organisations in partnership. This individual learning through interactions with people in partner organisations relies on the nature of their inter-personal relationships. By looking at such incidents, I should then be able to trace this individual learning (IL) to analyse how it is transferred to individuals in other organisations through their individual relationships (IRs). The mechanisms of relationships within organisations (ORs) will determine the extent to which learning is being encouraged as a field of organisational practice or hindered by the organisational structure. This may demonstrate how individual organisations learn (OL) through the learning of their members. The approach should then allow an analysis of processes of inter-organisational learning (IOL), if OL occurs following the interaction of members with colleagues from different organisations, which represents a form of interorganisational relationships (IORs). Figure 3 shows a model which describes relationships and learning at each of these levels within and between organisations. On the basis of the model, I hope to be able to look at individual learning styles, how individuals react to critical incidents, and their implications for individual and organisational learning. This leads, in turn, to an analysis of 
how IOL affects IORs as a consequence of learning and vice versa. A further issue is whether or not the processes of IOL and IORs are interwoven so as to connote aspects of partnerships, as shown Figure 4. For such an analysis, case studies have to be situated at the centre of the overlapping conceptualisation of IOL; IOR and partnership. Incidents selected for analysis should be associated with organisational structures and events and also with informal personal interactions.

Figure 3 Hypothesised process of IOR and IOL

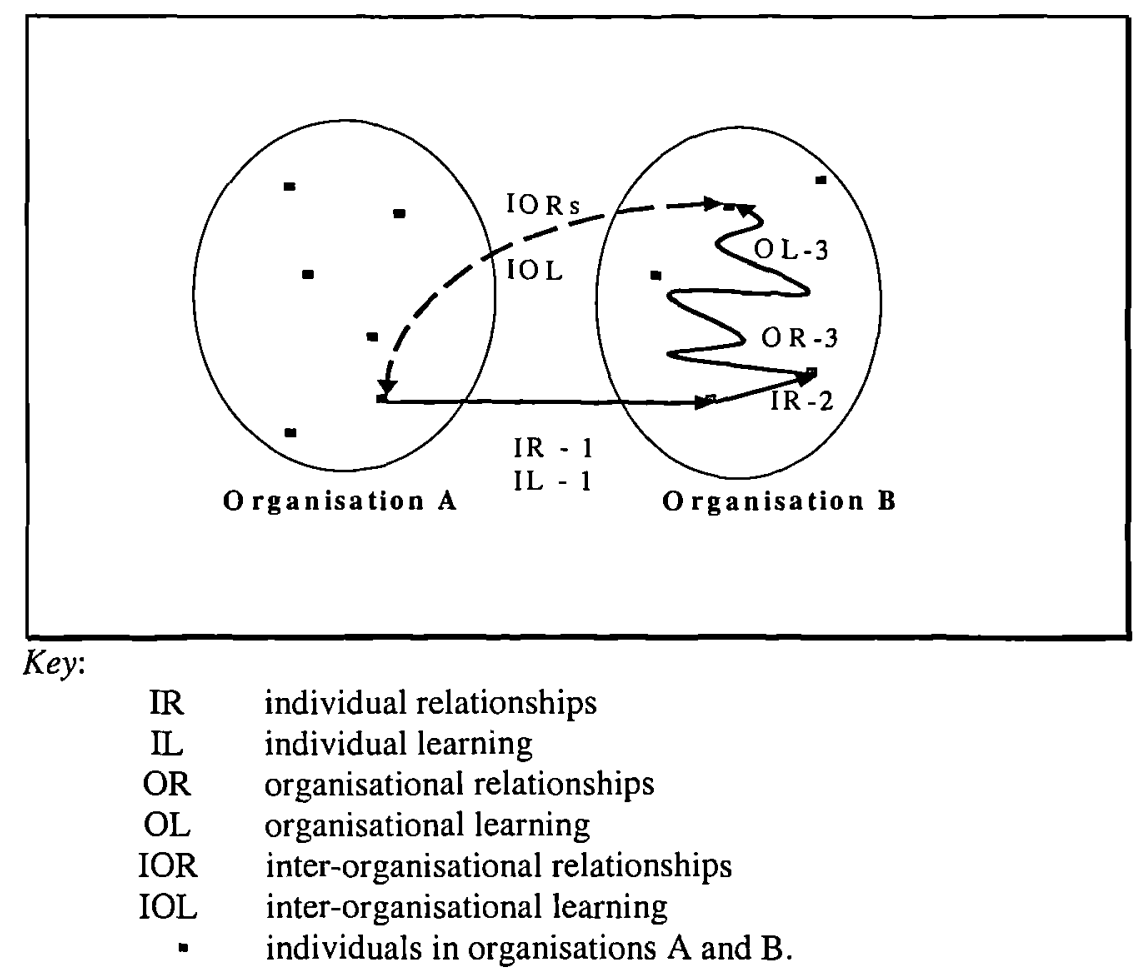

Note:

Numbers indicate the sequence of stages, which lead IL to IOL through OR and IORs. The same numbers illustrate occasions when learning and relationships emerge either at the same time or subsequently. The patterns described in Organisation B in Fig. 3 would be replicated in all members of the organisation network. 
Figure 4 Positioning case studies in relation to IOR, IOL and Partnership

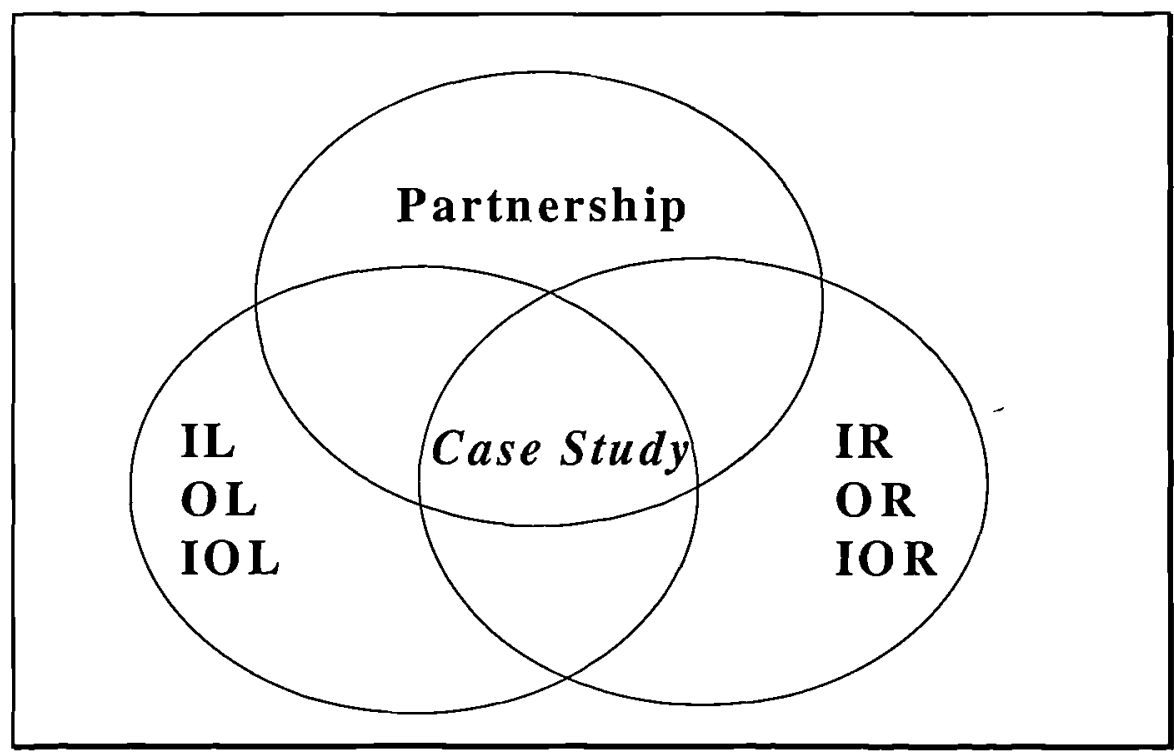

Note:

Circles represent the key conceptualisations. The case studies are located at the point where they overlap.

To prepare for an investigation of partnerships, studies of inter-organisational learning (IOL) are reviewed. These take account of learning and relationships, within and between organisations. Individual learning is the first kind of learning to be explored and then theories of learning in work-related organisational contexts are introduced. Following this, the analysis moves to the nature of intra- and inter-organisational relationships and how they affect individual and organisational learning to provide a conceptual link between the two. It is not possible to investigate leaning between organisations without first considering the factors determining the settings of IORs including their types and characteristics, since these determine the processes of partnership arrangements.

This chapter develops a conceptual framework for analysing the relationship between IORs and IOL in the aid sector. It points to inequalities associated with 
status differences between aid sector organisations. Having described critical elements relating to IORs and IOL, an account of the operational area of my research and case study selection follow. The difficulty of providing solid evidence of the connection between organisational relationships and learning has to be stressed. At best it is possible to give individual insights into the processes of learning in partnerships and an understanding of the roles and effects of learning on them and compare these with what others have to stay in similar circumstances.

\section{Learning within and between organisations}

Organisational learning is a consequence of individual learning. In order for an organisation to learn, its individual members also have to learn. We therefore need to consider theories of individual learning if we are to gain some insights into how individual learning translates into learning within and between organisations. Individual learning within organisations may be defined, according to Rollinson et al. (1998), as:

an experiential process which results in an individual displaying relatively permanent changes in underlying values of behaviour so that the individual is able to adapt to a changing environment. ${ }^{10}$

According to these authors, learning can occur only if a change in behaviour has occurred. It is important to notice, however, that the reverse does not necessarily happen. It means that people can temporarily change their behaviour, without any permanent change and therefore without any learning occurring. Jarvis

\footnotetext{
10 This definition draws on a number of important contributions in the individual learning literature, such as Argyris 1982; Kolb 1984; Argyris and Schon 1996.
} 
(1995: 58) suggests that definitions which emphasise a change in behaviour as an element of learning, are too restrictive, because "it would necessitate the exclusion of the acquisition of new cognitive knowledge unless it resulted in behavioural change". Argyris and Schön (1978), meanwhile, stress that individual learning within organisations not leading to radical changes may be seen to be as significant as learning that does occasion change. This will be discussed in greater detail in a later section of this chapter.

Different schools of thought espouse different theories of learning (Rollinson et al., 1998). Table 1 provides a brief picture of the key approaches and their assumptions. Classical conditioning theory and the behaviourist approach were originally developed through experiments on animals and subsequently extended to the study of human learning. Other theories have been developed to take account of social factors and individual differences going beyond the previous two approaches. The cognitive approach focuses on the role of memory in the acquisition, retention and retrieval of information, and the implications of these processes for learning ${ }^{11}$. The experiential approach, which develops and overlaps with the latter, aims to explain individual differences in learning by focusing on learning styles. Learning is defined as involving different stages, with each of these corresponding to a particular learning style. Different individuals have preferences for different styles of learning. Social learning

\footnotetext{
${ }^{11}$ For Anderson (1995), memory is a function combining acquisition, retention and retrieval. Acquisition refers to "how a permanent presentation of information is encoded and how this record is strengthened"; retention refers to how information is maintained in memory; retrieval refers to how information is brought out of memory when needed (191).
} 
theory integrates a behaviourist approach with an analysis of the role of the social environment and interpersonal skills for learning.

Table 1 Main approaches to learning and assumptions

\begin{tabular}{ll}
\hline Approach & Major assumptions and conclusions \\
\hline Behaviourist & Conscious learning, the recurrence of chosen behaviour leads to its \\
& reinforcement which follows it \\
Cognitive & Focus on the role of mind and instruction in learning \\
Social & $\begin{array}{l}\text { Focus on the role of environment in the learning process } \\
\text { Experiential }\end{array}$ \\
& $\begin{array}{l}\text { Analysis of individual differences in learning by focusing on learning } \\
\text { styles }\end{array}$ \\
\hline
\end{tabular}

Source: Adapted from Rollinson et al 1998: 214.

By applying an experiential approach, Kolb (1984) identifies different learning styles as those of concrete experimentation, reflective observation, abstract conceptualisation and active experimentation ${ }^{12}$. On the basis of Kolb's experiential learning model, Jarvis (1995) develops a model of the learning processes, which indicates the complexities of learning, as shown in Figure 5. Schön (1983) points to the capacity to reflect on action, so as to engage in a process of continuous learning as an inherent characteristic of professional practice. The idea of reflective observation is based on the second stage of the Kolb learning cycle. The concept of reflective practice will be further explored in the later sections with relevance to levels of the classifications and analysis made by Jarvis. Understanding types of reflective practice also provides useful insights into different stages of organisational learning.

\footnotetext{
${ }^{12}$ Honey and Mumford (1992) identifies preferences for experiencing, reviewing concluding from the experience and planning the next stage. This corresponds to activist, reflector, theorist and pragmatist personalities of the individual learner.
} 
Figure 5 Jarvis's learning model

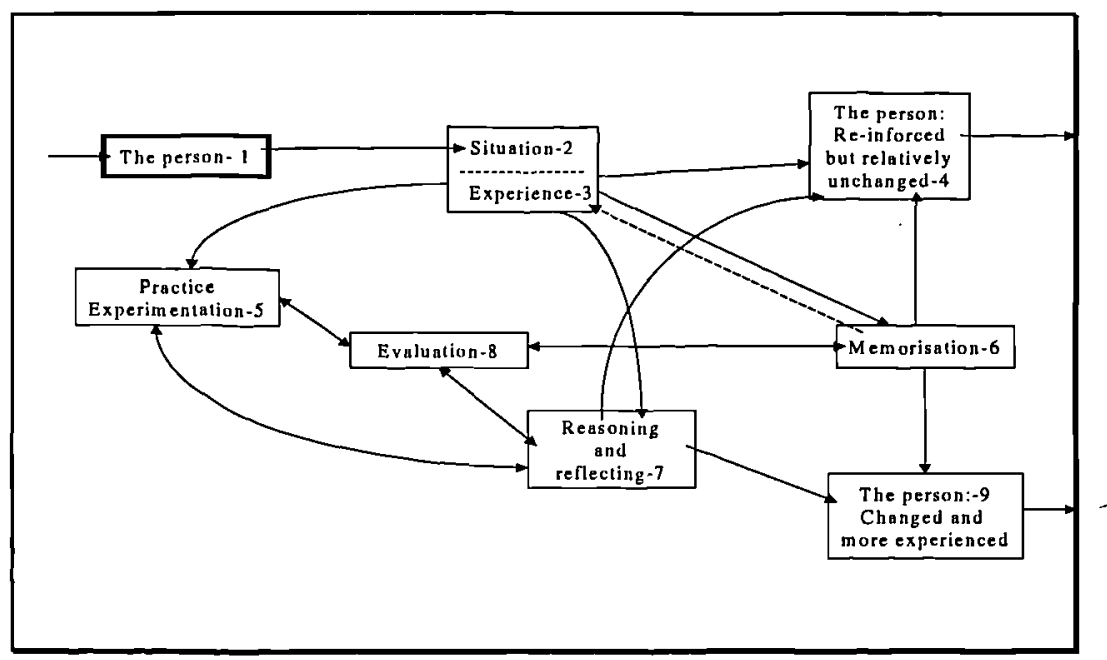

Source: Jarvis, 1995: 70.

Since different theories of individual learning focus on different aspects of the learning process, all are relevant to the process of organisational learning. Almost by definition, social learning theory is relevant to any analysis of organisational learning. Conditioning theories can explain why individuals follow particular behavioural patterns within organisations. Understanding the kind of stimulus to which individuals respond and the reinforcement which affects their behaviour can have implications for organisational learning. Cognitive and experiential theories go some way to explain how intellectual, cultural, psychological and individual differences, affect individual learning and translate into the learning processes of organisations.

According to Jarvis (1995), theories of experiential learning have become popular in recent years. Such learning may take place to large extent in the work place as an inherent part of an individual's work experience. In the community, 
learning takes place through daily experience. Jarvis (1995) analyses two modes of experience with reference to individual learning responses.

The [learning] experience is a subjective awareness of a present situation, the meaning of which is partially determined by past individual learning. Consequently, the experience is not merely of something external, but of the fusing of the external with the individual biography. (1995: 67-68).

The above notion of experience provides useful parameters for the analysis of work-related learning. It helps the observer to be aware of the complexity of determining whether individual learning emerges from current working experience or from previous learning experience. It also stresses the need to examine the external environment including the personal and social networks, where individual experience and learning occur.

Jarvis (1995) analyses types of learning on the basis of individuals' responses to their experiences. He identifies three categories: non-learning; non-reflective and reflective learning. Learning does not occur when people face a familiar situation or ignore and/or reject the potential for learning. Non-reflective learning occurs when people have experience without consciousness, acquire skills through imitation and role modelling, or rely on memorisation. Reflective learning involves processes of reflection, but is not automatically innovative or revolutionary. Reflective skill learning is the process whereby professionals in practice produce new skills as they respond to the uniqueness of their situation. Experimental learning is that " form of learning in which theory is tried out in practice and the end-product of the experimentation is a form of knowledge that relates fully to social reality" (Jarvis 1995: 74). This concept of experimental 
learning is relevant to organisational learning. Argyris's (1982) differentiation of single and double loop learning explains the difference between experimental learning, which brings innovative and change-oriented learning and other forms of learning, which do not bring about change. This will be explained later.

\section{Facilitating learning}

Learning occurs through both formal procedures and informal interaction. Formal learning maybe equated with training courses in organisations whereas informal learning is situated in everyday activities, which would include course participation. Lave and Wenger call this latter situated learning (Lave and Wenger 1991). Situated learning is usually unintentional and incidental rather than deliberate, and is particularly relevant to casual learning between individuals within and without the boundaries of organisations. Brown and Duguid (1991) emphasise the distinction between canonical and non-canonical practices within organisations. Canonical practices include official descriptions of how the various tasks carried out in an organisation, should be executed ${ }^{13}$. Canonical practices cannot detail the work of individual members of an organisation nor do they take into account the effects of changes in the environment. On the contrary, non-canonical practices are developed in order to solve day-to-day work-place problems. Non-canonical practices are usually crucial for the survival of the organisation, although the organisation might not be aware of them and might, indeed, refuse to recognise their significance. Brown and Duguid (1991) stress the importance of learning generated in

\footnotetext{
${ }^{13}$ Examples of canonical practice are corporation's manuals, job descriptions and training courses.
} 
informal communities-of-practice for the development of non-canonical practices. Within this framework, learning is not the hierarchical transmission of abstract knowledge, from someone who knows to someone who does not. Rather, learning is achieved through membership of the work place, and involves the individual learning by becoming an insider. According to Lave and Wenger (1991), who first introduced the concept of community of practice, it is defined as a set of relations among people and activity over time and in terms of its relation to other tangential and overlapping communities of practice.

Brown and Duguid (1991) argue that the boundaries of the communities in which this learning occurs are not necessarily coincident with the boundaries of the organisations. The concept of communities-of-practice can therefore be relevant in explaining the interaction between individual, organisational and interorganisational learning.

Araujo (1998: 317) regards learning:

As constructed within heterogeneous networks of social and material relationships that transcend and by pass conventionally defined organizational boundaries...... as being inevitably implicated in the everyday of collective practices responsible for the production and reproduction of organizations as well as an ingredient in the interactional practices that relate the organization to other actors, rather than a special practice associated with major change episodes or discontinuous innovation processes.

Araujo (1998) argues that knowing and learning are also dependent on the interorganisational and institutional structures in which organisations operate. $\mathrm{He}$ claims that 
these structures are important both for canonical, officially sanctioned relationships between organizations in the normal course of economic exchange and for non-canonical, informal relationships between individuals belonging to different organizations and institutions. The latter type of relationship provides opportunities for exchange of ideas, information, favours, etc., but also helps learning and search opportunities for new knowledge. In short, it helps participants in wider occupational communities to form consensual views on paths of technological development and to sharp search spaces for solutions to shared problems (Araujo 1998: 335).

Learning through informal interaction between individuals is thus seen to be just as significant as formally structured learning within organisations. Situated learning is often observed in the analysis of learning within and between organisations. Learning through social networks is identified as a significant factor in knowledge creation and sharing within organisations (Cross et al., 2001).

\section{Organisational learning}

In the analysis of organisational learning and the impact of individual learning, the theories illustrated above will be used to shed light on the selected case study learning experiences. Both individual learning and organisational learning are key factors in responding to uncertainty and rapid change. Individual learning requires a willingness to change, motivation to learn more and, most importantly, a willingness to embrace error. Organisational learning, requires a formal systematic process which enables individual learning to stimulate change in the existing system of an organisation (Finger and Brand, 1999b). 
The question of how individual learning links to organisational learning has been analysed by scholars in several areas. However, just as individual learning processes and their consequences vary, there is a complex array of processes and mechanisms of organisational learning. Stata (1996) sees organisational learning as the principal process through which management innovation occurs. $\mathrm{He}$ argues that organisational learning involves new insights and leads to modified behaviour, which differs from individual learning in several respects. He further explains that organizational learning occurs through shared insights, knowledge, and mental models (Stata 1996).

The terms "organisational learning" and "learning organisation" differ in the concepts they express. Accoring to Easterby-Smith and Araujo (1999), "organisational learning" focuses on understanding the processes involved in individual and collective learning inside organisations. "Learning organisation" refers to the development of methodologies for change through improvement of internal learning processes. Inter-organisational learning, here, refers to a dynamic process that involves individual and collective learning within and between organisations.

The field of organisational learning is divided into two broad schools of thought: the consultancy school and the academic school (Denton, 1998). Members of the consultancy school see organisational learning in terms of a positivist view of its potential benefits, whereas academic writers remain critical of the silence of the consultancy school on issues such as politics and power. Several authors 
analyse learning within organisations from various perspectives. Senge (1990) argues that learning is the result of a creative process of change that can transform an organisation. In The Fifth Discipline, he claims that five elements trigger learning within organisations: systems thinking, personal mastery, mental models, building a shared vision and team learning work. Kim (1993) focuses on mental models as an explanation of learning cycles between individuals and organisations. Later in this section, I shall refer to his model of learning cycles in order to explain a missing link between individual and organisational learning. The circulation of tacit and explicit knowledge in organisations for the sake of learning and knowledge creation are widely recognised, with evidence from Japanese companies provided by Nonaka and Takeuchi (1995).

Antonacopoulou's (1999) analysis of the obstacles to management learning in the light of personal and organisational differences provides some account of the factors that stimulate individuals and organisations to learn.

Table 2 Personal and organisational influences on learning

\begin{tabular}{ll}
\hline Personal factors & Organizational factors \\
\hline Perception of the need to learn & Internal organisation of work \\
Perception of ability to learn & Organization systems, e.g. training \\
Cultural values and beliefs & Culture and climate \\
Emotions-feelings/reactions & Decision-making processes \\
Attitude towards updating & Communication and feedback \\
Intellectual-mental capability & Politics and aversion to risk \\
Age & Instability and change \\
Memory & Economic position, competition \\
Ability to communicate & Power and control \\
\hline
\end{tabular}

Source: Antonacopoulou 1999: 222.

Table 2 lists elements, which facilitate learning for individuals and organisations. Here, attention needs to be paid to those elements which exist within and 
between organisations, as well as at the level of individuals, in order to understand how learning occurs between various actors beyond individual organisations.

Although organisational learning has existed for decades as a topic in the study of management, its practical importance has only been recognised in the past ten years (Easterby-Smith and Araujo, 1999). One of the reasons for the increased interest in this topic is that it is being emphasised in business. The ability of organisations to learn faster than their competitors is thought to be a key factor for successful business. Learning in business has become a normal process, in order to produce, transform, and implement information faster than competitors in a context of intense competitive pressures (Finger and Brand, 1999b). The learning organisation is recognised as "adaptation" or "reaction" to environmental pressures leading organisations to change (Easterby-Smith and Araujo, 1999)

\section{Relations and Learning in Organisations}

There are two levels of learning in organisational studies. Argyris and Schön (1978) refer to them as single and double loop learning, while Levinthal and March (1993) label them "exploration" and "exploitation". Single-loop learning is related to incremental changes, whereas double-loop learning is linked to radical changes. This distinction, drawn by Argyris and Schön is highly regarded by managers and theorists, as it provides an effective explanation of modes of organisational change. In single loop processes, learning occurs as a means of 
correcting the mismatch between intentions and what actually happens, and by changing the behaviour or the actions involved. Therefore, people learn to achieve a goal without questioning the values and assumptions that underpin the goal.

In double-loop processes, such a mismatch is detected at the outset, and behavioural change occurs subsequently as a means of correcting the mismatch, while the values and assumptions underlying the goal are monitored and changed. As Argyris (1982) stresses, organisations learn to learn only if the double-loop learning process is achieved. However, there are difficulties in defining adequately the forms of change which are associated with learning, or indeed, clarifying when single or double-loop learning is appropriate. Along with this, Levinthal and March (1993) note the dilemma between exploration and exploitation in the use of technology. Easterby-Smith and Araujo (1999) stress that organisations have to seek a balance between the two. However major problems emerge when a decision is based on a political agenda rather than on a rational calculation.

Coopey (1996) claims that without considering power and power relations in organisational culture and structures, the achievement of a learning organisation remains a romantic and rhetorical ambition. The extent to which this is the case and its implication for the smoothness of organisational change are important here. The organisations I consider are not in the private sector, but are government, non-government and voluntary organisations. Finger and Brand 
(1999a) differentiate organisational learning in the private sector from that in the public sector, claiming that public sector organisations operate in a more problematic environment which brings added complexity to their functions and management. They point out that some characteristics of the public sector prevent change. These include a deeper bureaucracy compared with that of the private sector, its function in the political environment, and the difficulties it encounters in introducing reform across society. They stress the significance of analyses of internal and external organisational relationships and of specific characteristics of organisations. All this has implications for my study.

Kim (1993) provides useful insights into the mechanism of transformation of individual learning into organisational learning by using mental models. The mental models he uses represent individual and organisational views of the world, including their explicit and implicit understandings. He argues that most of an organisation's knowledge (both know-how and know-why) lies inside peoples' heads. According to Kim, "organisational learning is dependent on individuals improving their mental models; making those mental models explicit is crucial to developing new shared mental models" (ibid). He identifies three types of incomplete learning cycles that affect organisational learning: situational, fragmental and opportunistic. Situational learning occurs when individuals do not codify learning for later use, so that there is no obvious link between individual learning and individual mental models. For instance, when a crisis occurs, an individual may solve a problem, but not applying the lessons learned to the next one that occurs. Fragmented learning occurs when no linkage 
can be seen to connect individual mental models and shared mental models. This remains the case when individuals leave organisations, which then lose their accumulated wisdom. For instance, decentralised organisations without network capacities to keep the parts connected, tend to experience fragmented learning. Opportunistic learning happens when organisational actions are taken on the basis of an individual's or a small group of individuals' actions, but not on the basis of the organisation's shared mental models. In applying Kim's model of incomplete learning cycles, inter-organisational learning can also be explained at the level of what happens between individuals, and between organisations, and of how the learning in each case leads to organisational learning.

I shall refer to data based on individual experiences. This means that instances of organisational learning will not necessarily be clearly identified. For the purposes of this research, the focus of the literature considered so far in this chapter has been on individual learning within and between organisations, its processes and constraints. The challenge is to see how the individual learning experiences outlined lead to organisational learning, as well as how they affect the nature of inter-organisational relationships.

\section{Relationships and Learning between Organisations}

Little research has been done to extend organisational learning theory to an interorganisational level (Larsson et al.,1998). The study carried out by Larsson et al. (1998) aims to develop a process-oriented conceptual framework of interorganisational learning in strategic alliances, and its implication for success or 
failure in developing collective knowledge. The focus of this section will be on some of the determinants of IOL, with reference to the mechanisms of IORs. The relations between learning and the formation of organisational relationships are examined first in the context of business alliances, some elements of which are regarded as crucial to the processes of learning between organisations. A number of researchers have already stressed the importance of taking account of both national and organisational cultures (Pollard, 2001), and also of occupational and other sub group cultures within organisations (Levinson and Asahi, 1995). Awareness of these levels of culture and of their interactions can be a leading factor in determining whether inter-organisational learning is promoted or hindered (Levinson and Asahi, 1995).

Pollard (2001) also emphasises management-style differences and strategic imperatives as having a significant influence on collaborative processes, such as the transfer of information and learning in the practice of joint international ventures. He states that learning to work effectively with a partner company's culture, management style, and operational differences poses a number of learning opportunities for both management and staff (Pollard 2001: 76). The role of the manager in learning about a partner organisation is often regarded as revealing a sign of the degree of commitment to stimulating learning in alliances (Sohal et al., 1998). Trust and commitment to co-operation can be effectively promoted through forming a learning alliance, as key to strategic alliances (Holt et al., 2000). 
Several steps in securing the transfer of knowledge in inter-organisational learning are identified by Levinson and Asahi (1995): identification of new knowledge, knowledge use to achieve intended outcomes and the institutionalisation of knowledge by reflecting on what is happening and adjusting alliance behaviour. The authors claim that these complex interactions involve culture, structural patterns and work systems. Levinson and Asahi (1995) stress other factors which influence IOL, including strúcture, technology and absorptive capacity, which is the ability to select new ideas and adapt to them. A network analysis of how organisations relate to each other may provide a picture of information flows and learning which enables the position of organisations within the network to be better understood. For instance, if an organisation is positioned at the centre of the network, its role in IOL must be active. Levinson and Asahi also emphasise the brokering role, at multiple linkage points, of personal attachments. These enhance the acquisition of new resources, as well as the identity and communication of new knowledge. As for technology-based IOL, they claim that there must be sufficient prior trust and respect for this to be achieved. Most importantly, absorptive capacity, both for organisational and inter-organisational learning, is a crucial factor in the successful introduction of planned change.

There is, therefore, a case for a study of the importance of learning and the effects of learning on the quality of multi-organisational interactions. In particular, organisational culture, management and strategic approaches have a significant impact on the analysis of how organisations learn from each other. 
There is also a suggestion that understanding differences in partners' organisational mechanisms, including strategy, work systems, the culture of small groups and technical capacity, plays a leading role in stimulating learning in co-operative relationships. Comprehending the nature of the networks in which partner organisations are located and the directions of their resource and information flows can help develop a strategic approach to the creation of interorganisational linkages. Gnyawali (1999), for instance, analyses the relationships between network and learning from a perspective of interorganisational learning. This provides useful insights into the impact of organisational linkages and degrees of learning. Social networks, which are established both formally and informally by individuals from various backgrounds and organisations, cannot be ignored as a learning resource between organisations. In this study, the approach of communities of practice, provides the parameters of an analysis of inter-organisational learning. Individual interaction, which contributes to establishing trust-based relationships between organisations, plays a significant role in structuring learning opportunities.

\section{Exploitation of mutual learning in partnership}

While there is a significant link between learning and the effectiveness of alliances, learning may not always work as a positive factor in developing IORs. Larsson et al. (1998) point out that the focus of contemporary research on IORs is on how to be a good partner in strategic alliances or on how to win the internal race to learn among the partners. They develop a framework based on questions of how collective learning is developed in strategic alliances and how joint 
learning outcomes are distributed variously among partners. They analyse types of learning strategy, designating them as collaboration, competition, compromise, accommodation, and avoidance. These terms are based on how receptive and transparent an organisation is to partners. According to their analysis, in the case of collaboration, an organisation is highly receptive and transparent. In the case of competition, the organisation is highly receptive, but not transparent. When the organisation is moderately receptive and transparent, its strategy is compromised. When the organisation is not receptive, but highly transparent, it is accommodating. Finally where the organisation is neither receptive nor transparent, the strategy is avoidance.

Larsson et al. (1998) propose that IOL outcomes are interactive results of the receptive partner's type of adopted learning strategy. From their analysis, it can be seen that organisations are most likely to learn together when all the partners choose collaborative learning strategies of high transparency and receptivity. However, the emphasis on good partners' advice on the integrative dimension of maximum joint learning tends to overlook the extent to which the individual organisations may appropriate such joint learning (Larsson et al., 1998: 300). They describe the dilemma of inter-organisational learning thus:

(1) being a good partner invites exploitation by partners attempting to maximise their individual appropriation of the joint learning, and (2) such opportunistic learning strategies undercut the collective knowledge development in the strategic alliance.

Larsson et al. conclude that IOLs can be hindered by lack of either motivation or the ability to absorb and communicate knowledge between partner organisations. 
They claim that the dynamics of power, opportunism, suspicion, and asymmetric learning strategies can constitute barriers to collective knowledge development. In contrast, they suggest that the prior related interaction between partners, high learning stakes, trust and long-term orientation is likely to empower the collective learning process (285).

The points Larsson et al. (1998) make are mostly appropriate in a situation where organisations in partnership have diverse objectives, capacities and status. This is often the case in the aid sector. These diversities become a particularly significant element for cross-national and cultural arrangements as Tayeb's (2000) International business partnership illustrates. Problems emerging from business practice are assumed to be the issue in partnership arrangements in the aid system where multi national and cultural organisations are involved. The analysis of the elements that can create barriers to collective learning development thus provides insights into the gap between individual learning and organisational learning.

\section{Learning in development agencies}

The term learning organisation is being more and more frequently used, not only in business-oriented organisations, but also and most recently, in development aid agencies. This section introduces some writing on aid sector learning policy and practice. 
The DAC report Evaluation Feedback for Effective Learning and Accountability indicates the unsatisfactory performance of DAC agencies in learning from lessons in development practice (OECD, 2001a). The report emphasises development for optimum usage of evaluation systems and accountability. This is reflected in the recent pressure for aid agencies to become learning organisations and knowledge-based organisations. This is a significant element in the current aid development discourse, headed by the World Bank (Barnard 2002). The World Bank contrasts three types of learning: learning from doing; learning from research and learning from evaluation (ibid).

The DAC report identifies some of the obstacles to learning. These include: organisational culture; pressure to meet disbursement targets; lack of incentives to learn; tunnel vision; loss of institutional memory; insecurity and the pace of change and the unequal nature of the aid relationship. Denning (2000) suggests using story-telling to explore learning accounts in the World Bank. He argues that story-telling enables change by instructing the living part of the organisations. By story-telling, individuals in an organisation can see themselves and their organisation in a different light, take decisions and change their behaviour in accordance with these new perceptions, insights and identities. The idea of story-telling to induce organisational change is close to the thinking on communities of practice. It also reflects the idea of shared mental models, which Kim (1993) proposes as a means of reducing the gaps in learning between individual and organisational levels. Techniques of story-telling may well be 
relevant to my intended analysis of individual learning accounts and their influences on organisational learning processes.

The research of King and McGrath (2002) on the knowledge management policy of multilateral and bilateral agencies provides the most recent account of approaches to research on issues of concern here. Comparing JICA, DFID, SIDA and the World Bank in the light of the phenomenon of "knowledge-based aid" (King, 2000), the study illustrates agency differences in terms of knowledge discourses, knowledge products, knowledge projects and knowledge practices (King and McGrath, 2002). One of their findings is the inconsistency of the knowledge-based approaches across new projects with little direct concern for their impact on poor people's development.

The Swedish bilateral aid agency (SIDA) has undertaken studies of its own regarding organisational learning. The study carried out by Forss et al., (1997) for the Swedish Ministry for Foreign Affairs, provides a detailed analysis of organisational learning in a bilateral development co-operation organisation. In their analysis, external pressure is seen as a major driving force behind learning and change. Other aspects hindering learning include structural aspects of learning, process-related obstacles to learning and cultural aspects of learning.

Carlsson and Wolfgemuth (2000) provide a collection of individual accounts of learning in the various practices in the aid sector. In their concluding remarks, they identify some factors which make learning difficult in the aid sector. These 
are political constraints; the unequal nature of the aid relationships; problems internal to the organisation of aid agencies; the organisations and capacities on the recipient side and sources of knowledge and the quality of information.

The concepts of the learning organisation and organisational learning derive from organisational management strategy, and imply practices such as capacitybuilding and organisational development (Eade, 1997: James, 1998). In the NGO sector, the pressure for NGOs to become learning organisations has increased (Britton, 1998). Organisational learning programmes have been provided principally by British Overseas NGOs for Development (BOND). A BOND discussion paper encourages NGOs to analyse their activities in terms of learning organisation constraints and their implications for outcomes (O'Malley and O'Donoghue, n.d.). An NGO support organisation, the International NGO Training and Research Centre (INTRAC) has taken the lead in providing specially designed management, training and research services for NGOs internationally, since its establishment in 1991. Edwards (1997), too reflects on NGO experiences as learning organisations.

\section{Practices of learning in development co-operation}

The above studies have highlighted the increasing awareness of developing a learning culture in the aid sector. However the inequalities between organisations in the aid sector are also emphasised as influential factors in the processes of learning. The complexity of development co-operation affects learning within and between stakeholder organisations (Forss et al., 1997; 
Carlsson and Wolfgemuth, 2000). In the aid sector, environmental turbulence, which means the level of uncertainty in the performance of a task, is higher than in other organisations. This is the result of rapid change and unpredictable social events that are affected by conflict and competition of interests, under intense and often hostile public scrutiny (Forss et al., 1997). This situation puts pressure on decision-makers to obtain more information. Knowledge levels remain high, and as a result, learning needs are also high. On the other hand, some of the complexity of development cooperation, such as uncertainty about goals and objectives, the uncertainty of feedback mechanisms and high transaction costs can prevent the development agencies from learning. Forss et al. (1997) comment that actors in development co-operation are part of a national administrative environment, in many cases moulded on the bureaucratic form of organisation which often prevents those working in development co-operation from learning (Forss et al., 1997: 1-2).

The inequalities highlighted in IORs, in the aid sector, constitute asymmetric organisational relationships. These are associated with varying organisational capacities, which include financial, human and technical resources; organisational linkages with other organisations; and the status of organisations. The inter-organisational relationships to be described in this thesis are between donor and recipient organisations and between these and technically collaborating organisations. Each organisation can be portrayed as having particular advantages over the others. Donor agencies have financial advantages, while local implementing organisations have local knowledge and expertise as 
advantages over the donor. By maintaining such advantages in the process of development interventions, inter-organisational relationships can cause blockages to learning from each other. Each actor has his or her own advantage over the other to a greater or lesser extent. Each of them also struggles with the dilemma of whether to benefit from the learning opportunities they encounter or ignore them, consciously and unconsciously, so as to proceed with the routines of their bureaucratic structure.

A number of studies show relational advantages to be an obstacle to participation, where learning is most likely to emerge. Donor agencies tend to blame their recipients for project failures, whereas they tend to congratulate themselves for any successful cases (Eade, 1997; Isezaki, 1997). This implies that reflection and learning from experience is less a feature of donor agencies. Non-learning organisations develop a proficiency in ignoring problems and solutions to problems by replacing problematic issues with something else (Brunsson, 1997). The particular structure and nature of the relationship between donors and recipients needs to be considered in order to identify factors which hinder learning. Eade (1997: 192) claims that:

...the donor-recipient relationship is not one that lends itself to reciprocal learning. The structure and bureaucratic mechanisms an agency requires to administer funds are often quite different from (and even inimical to) those needed in order to identify and act on critical insights from the recipients of those funds.

Several authors acknowledge the need for transformation in the culture of aid agencies for them to become learning organisations. Korten (1980: 498) sets out 
some requirements for them to become learning organisations: to embrace error; to plan with the people; and to link knowledge-building to action.

Learning difficulties also arise from the nature of the work in the aid sector. They include the notorious difficulty of measuring learning outcomes and effects, high centralisation of management, mobility of staff with acquired knowledge, and hierarchical flows of information in public administration (Carlsson and Wolfgemuth, 2000).

The case study in technical co-operation examined by Forss et al. (1998) finds an unbalanced relationship between learning for the purpose of efficiency and learning for the purpose of development at large. Their case study shows that structures and processes for improving the efficiency of technical co-operation may have an adverse effect on learning. They also note that the existing system meets the different purposes of learning to varying degrees. For instance, as emphasised in their case study, the development community and aid agencies are proficient at learning to improve efficiency, as illustrated by the evaluation function and the development of procedures and practices codified in various manuals in technical cooperation. Forss et al. state that: "(I)t would thus appear that the development community has better learning systems for purposes of less importance, and poorer learning systems for purposes of more importance" (Forss et al., 1998: 53). 
Some characteristics of bilateral aid organisations, which may influence learning include the kind of political environment in which such organisations work and exist in relation to other organisations in their networks. The nature of these relationships influences their policy and implementation strategies, directly and indirectly. Analysis at this level also involves detailed understanding of the background of organisations, such as an individual organisational history. At another level, emphasis needs to be placed on the culture of learning in organisational analysis. How internal organisational mechanisms and the interests of bilateral donor can both facilitate and obstruct learning is considered. Factors for analysis include the internal and external pressure on evaluation and the structures of management systems.

The forms of inter-organisational relationship relevant to their research, include donor-recipient relations, state and non-governmental relations, horizontal organisational networking, and hierarchical collaborative relations. Bilateral aid organisations are situated as one set of a country's foreign policies. Their objectives are primarily determined by their government's political and economic imperatives of their relationships with other governments, rather than by a heartfelt commitment to poverty alleviation.

However, development agencies, particularly those implementing projects/programmes in recipient partner states have to deal with conflicts between their own organisations' purposes and what may be most appropriate at the field level. This multiplicity of intentions at different levels of development 
co-operation is unlike that of the corporate culture, where the primary common interest lies in profit. As a result, the complex mechanisms of development aid practice are constituted by the multiple forces of the different organisations' individual intentions. Bilateral aid organisations, by definition, function on the basis of inter-organisational relationships across cultures, nationalities and organisations with diverse status, purposes and backgrounds.

The most recent partner in the aid sector is the non-government organisation (NGO) community, both national and international. As discussed in the previous chapter, NGOs have become one of the most significant vehicles through which bilateral aid organisations achieve their various goals. The types of relationships created by these two organisations vary with mechanisms of funding. Today the principal options are direct funding and contracting-out. The roles of NGOs include advocacy lobbying and identifying local partners for government. The increasing number of occasions on which they meet to exchange ideas and information, often under the name of partnership, are likely to be a factor for changing their relationships, at least in a visible way. Forss et al. (1998: 55) comment on learning and partnerships from their perception of the contribution each makes to organisational changes.

(l)earning which does not occur in partnerships is not likely to lead to any meaningful change in practice, and hence there is no real organisational learning as we have defined it here. Power is unavoidable, and the partners in co-operation bring different aspects of power to the negotiating table. There is a need to constantly monitor the way power is used, and to consider when it becomes an obstacle to learning.

Learning must permeate the relationship between the partners in development, and it should be an integral instrument in creating effective, 
relevant and sustainable results of co-operation (ibid., executive summary).

\section{Research issues, plans and methods}

While I was reviewing the literature on learning from the perspective of both IORs and IOL, I began to look for case study human development projects and organisations through which to explore the issues discussed in the proceeding pages. My concern in respect of learning and partnerships in the aid sector is the relationships between organisations working in partnership, in terms of their asymmetric advantage and the assets and elements which determine the power they wield. As a transnational service provider, national and cultural aspects of individual organisations' characteristics, the structural processes of learning and the dissemination of knowledge across the partnership in the aid sector must also be considered. To analyse these issues in an international system committed to poverty alleviation, a comparison of partnerships managed by different organisation seems to be a suitable approach. Such a study will lead me to understand similarities and differences between projects, in terms of how their members interrelate and learn from each other. The projects chosen have to be structured and managed differently so as to be able to compare the national and cultural aspects of the organisations involved. Such considerations led me to the decision to investigate multi-organisational projects funded by different donor organisations. To increase their comparability and reduce the descriptive complexity and cost, it would help if two projects funded by different donors and operating at the same time, with similar community features, could be found in 
the same country. Before identifying such projects, it seems appropriate to describe the characteristics of the two funding organisations that will support such an enquiry.

My interest lies in the process of learning within and between organisations. This requires an analysis that will take into account the development of an activity at various stages, across the hierarchical structures of partnerships. Therefore the projects chosen have to allow for an account of how the projects were planned by the funding agencies and the managing organisations and how they are positioned in their wider policy agendas. The projects have also to permit me to investigate how they are being implemented on the ground. IOL has emerged from the literature as a leading element in enhancing partnership. It may also be strategically planned to win a competitive learning race, in many cases behind a mask of cooperative relations. Taking this into account, my study has to situate itself in a position to observe both sides of the process of interorganisational learning. The analysis will take account of the diversity of culture, external international forces and management systems to develop the critical insights of asymmetric relationships. The unevenness of power relations as an element of IORs will be taken as a central concern in the study of learning in partnerships. Inevitably in the aid sector partnerships, one of the organisations involved is dominant. The challenge is first to see how the policy and practice of partnership disguise its inequality and then, to see how the roles and effects of learning dance between layers of organisational hierarchies and inter- 
Chapter 2: Learning and organisation

organisational relations. This will require an analysis of obstacles to partnership learning. 


\section{CHAPTER 3 DEVELOPING THE FIELD RESEARCH: BACKGROUND TO THE CASE STUDY PARTNERS}

\section{Introduction}

The organisations used as case studies within this research are now introduced. Attention is focused on differences in the quality of their internal relations and on their relationships with partners.

A combination of factors led to the selection of DFID and JICA as the lead organisations of possible case study projects. My skills had been developed in Japan and the UK and I speak Japanese and English fluently. I have written about their role in education for development and done a consultancy for JICA about DFID's project management procedures. To reduce contextual variation, I set out to find two projects with local NGO implementing organisations, one funded by each organisation that were being delivered in a single country, where English was widely spoken. In Africa, Malawi and Ghana were the only two countries in which DFID and JICA were both working and met each of the other criteria. I chose Ghana. DFID's local counterpart non-government organisation (NGO) was the Ghana Institute of Linguistics, Literacy and Bible Translation (GILLBT) and JICA's was the Planned Parenthood Association of Ghana (PPAG). The DFID partnership with GILLBT was for an adult literacy project. The JICA partnership with PPAG was a maternal and child health care project. 
Each of the two projects was also linked to skills for livelihood incomegeneration schemes.

The information in this Chapter presents the two funding and counterpart organisations in the context of relevant aspects of social development in Ghana. Information was taken from published and unpublished documentation and primarily from interviews with key informants. Citations from these interviews are referenced according to the number of the transcripts to which they refer. A list of transcript to be found in the Appendix 1

\section{DFID}

The history of the UK aid serves as useful background to current DFID policy and responsibility in relation to other UK government departments. In 1929 the Colonial Development Act acknowledged continuing responsibility for the development of its colonies (DFID, 2001a). In 1961, the Department of Technical Co-operation was set up to deal with the technical co-operation side of the aid policy. In 1964, the Overseas Development Ministry (ODM) was established as an independent ministry. The ODM took responsibility both for the functions of the former Department of Technical Co-operation and for the overseas aid policy functions of the Foreign, Commonwealth Relations and Colonial Offices, as well as other government departments (ibid). In 1970, the functions of the Minister for Overseas Development were moved to the Secretary of State for Foreign and Commonwealth Affairs. At this time, overseas development work was managed by the Overseas Development Administration 
(ODA) as "a functional wing of the Foreign and Commonwealth Office" (FCO)(ibid). Although ODA was declared an independent ministry in 1974, it returned to work under the FCO in 1979. In 1997, the ODA was replaced by the Department for International Development (DFID), headed by a Secretary of State, formally responsible to Parliament for DFID. The DFID mission statement reads as follows:

The Department for International Development (DFID) is the UK Government department responsible for promoting development and the reduction of poverty. The government first elected in 1997 as increased its commitment to development by strengthening the Department and increasing its budget (DFID, 2001a).

Despite having been repeatedly merged with the FCO, the British aid system maintained relative autonomy from the FCO. However, this affiliation may also have helped British aid agencies to avoid involvement with incompetent or corrupt regimes in Africa (Lancaster, 1999: 140). DFID works with: the Department of Trade and Industry on trade issues; the Ministry of Defence and FCO on conflict prevention and post conflict reconstruction; the Treasury on debt; the Department of Transport, Local Government and Regions on environmental issues and the Department for Education and Skills on child labour (DFID 2000b).

As Lancaster (1999: 133) explains, despite the British government's initial intention of not assisting former colonies after independence, pressures from the governments of America and Canada had an impact on the decision to commit to providing significant aid to its former colonies in Africa. In its early stages, British aid policy was characterised by some degree of flexibility and by a lack 
of development doctrine, strategy, and focus. Following this, as with other donors, the UK focused on rural development in the 1975 White Paper, while in the 1980 s economic policy reforms were given priority. The aid policy of the 1990s focused widely on four categories: the promotion of the market and good government; health and education, with an emphasis on women; the protection of the environment and support to multilateral development institutions for sustainable development. The current policy, as revealed in the 1997 White Paper Eliminating World Poverty, is characterised by a commitment to the international target of halving the number of people in extreme poverty by 2015 . This target was associated with other targets including basic health care provision, universal access to primary education and more equal opportunities for women. The policy to emphasise poverty alleviation continues to be stressed in the most recent White Paper 2000, which also focuses on managing the process of globalisation to benefit the poor (DFID, 2001a).

\section{Partnership policies}

A quarter of the 1997 White Paper was dedicated to the discussion of partnerships. This shows the strength of government commitment to partnerships as a means of achieving the target to 'eliminate poverty'. The policy of enhancing partnerships, in the public, private and voluntary sectors was stressed in 1997 by the newly elected Government (IPPR, 2000). The concept of partnership in the White Paper is considered in respect of different development agencies: multilateral organisations, bilateral organisations and British partners, including public and private, business and voluntary sector organisations. The 
White Paper stresses the importance of working closely with other donors and development agencies to build partnerships with developing countries and to combat poverty. DFID will use its influence to mobilise the political will to achieve these international development targets. The UK aid policy represents new modes of partnership: working with UK private and voluntary sectors and with the research community. The Government also wishes to strengthen its partnership with voluntary, charitable and non-profit organisations. DFID plans to work in alliance with the voluntary sector in order to win strong public and international support for poverty elimination and sustainable development.

The White Paper states:

The Government believes that genuine partnership between poorer countries-including developing countries and relevant middle income countries such as countries in transition and Dependent Territories- and the donor community are needed if poverty is to be addressed effectively and in a coherent way. The establishment of such partnerships moves beyond the old conditionalities of development assistance and will require political commitment to poverty elimination on both sides....(DFID, 1997: 37).

However, some authors are sceptical about the fact that the adoption of the concept of partnership implies a real change:

while the name now given to development co-operation may be partnership, the game is to be played according to rules that the donor has decided in advance. Not much change there. The partnership is still very much about donors exercising influence within a structural relationship of asymmetrical power, even if the application turns out to be less coercive than the cruder forms of leverage associated with the old practices of aid conditionality and the Cold War (Burnell, 1998: 798799).

The Government continues to support British voluntary agencies through a number of programmes. In 1996, 9.3 percent of UK's aid went through UK 
NGOs to low-income states (Smillie and Helmich, 1999:239). Of this, one-fifth went to the Joint Funding Scheme; one third went to emergencies; 14 percent was used by agencies to send volunteers overseas and one-third was part of bilateral country programme funding that went through NGOs (ibid). The Joint Funding Scheme was the original source of ODA funding to UK-based NGOs throughout the $1980 \mathrm{~s}$ and $1990 \mathrm{~s}^{14}$. The scheme was replaced by the Civil Society Challenge Fund (CSCF) in 1999, which was managed by the Civil Society Department $(\mathrm{CSD})^{15}$. The DFID case study project in this research is operated under this scheme.

Regarding aid to the education sector, the policy framework states that 'DFID will assist the strengthening of education systems through partnerships that build in a sustainable way on local and national initiatives' (DFID, 1997:7). The framework also states:

Relationships with education ministries are taking new forms. Partnerships are being forged with government; NGOs, civil society organisations and other bilateral agencies in support of sector-wide change and reform. Local level partnerships are critical for the success for this process. New codes of conduct, instituted by governments and development agencies together, are being put into place (ibid: 8).

That new DFID sector-wide reform support may imply reduced support to project-based NGO activity, was a worry expressed by one NGO worker. However the full implications for NGOs are not yet clear, according to the DFID officer (Transcript 2).

\footnotetext{
${ }^{14}$ The Joint Funding Scheme was DFID's principal means of supporting NGOs' long term overseas development project. An applicant must be an UK registered charity and relate to projects in which the intended beneficiaries are the poorest communities in low-come states. Grants are offered up to $£ 500,000$ and usually co-funding on a 50:50 basis.

${ }^{15}$ Chapter 5 describes detail of CSCF.
} 


\section{Japanese ODA and JICA}

In terms of its historical background, Japan is unique among aid giving countries. Japan started the post-war period as a major recipient of foreign aid: $\$ 5$ billion were received from the United States during the period from 1946 to 1951, and thirty-four loan schemes worth $\$ 860$ million were received from the World Bank. Japan completed repayment of these loans in July 1990. Japan established its own medium-term ODA plan with quantitative goals in 1976, after completing payments for war reparation to Asian countries. In 1989, Japan became the largest national donor of bilateral foreign aid. In 1994, Japanese ODA funding amounted to twenty two percent of the total development assistance provided by OECD/DAC countries (Fujisaki et al.,1997). The decline of aid in other OECD countries was reflected by their own fiscal problems (Chapter 1).

Japanese ODA has a complex structure, involving a number of actors. Although the Ministry of Foreign Affairs (MOFA) is the body responsible for ODA policies, the Ministry of Finance (MOF), the Ministry of International Trade and Industry (MITI) and the Economic Planning Agency (EPA) pursue their own economic agenda in ODA. Other ministries, the private sector, and a wide range of public corporations sponsored by the ministries, are also involved in ODA funding (Beaudy-Someynsky and Cook 1999). The delivery system of Japanese economic co-operation remains the same as at the time of its creation in 1953, despite shifts in policy focus. Initially, a council of eleven ministries worked on war reparations and economic co-operation. The system was then streamlined to comprise the four entities: MOF, MOFA, MITI and EPA. The Foreign 
Economic Cooperation Advisory Council, chaired by the Prime Minister included prominent business leaders. It established the present system of supporting organisations in 1961: the Japan International Co-operation Agency (JICA), the Overseas Economic Co-operation Fund (OECF), and the Institute of Developing Economies (IDE) ${ }^{16}$ (Beaudy-Someynsky and Cook, 1999). In 1999, the Japan Export-Import Bank (JEXIM) and OECF merged into the Japan Bank for International Co-operation (JBIC), and this is responsible for the extension of government loans, as well as for providing grants and loans to companies engaged in projects in developing countries (MOFA, 1999: iv). MOF, MOFA, MITI, EPA, OECF, and JBIC are the main actors in economic co-operation and JICA is the institution responsible for co-ordinating technical co-operation.

\section{JICA as executive agency for Japan ODA}

The Japan International Co-operation Agency (JICA) is an agency for managing government-based technical assistance. JICA also conducts training programmes in Japan for people from low-income states. It runs an expert dispatch programme for which it provides equipment and material. JICA is under the authority of MOFA, which controls the budget and disbursements, and defines overall foreign policy. Until recently, the Japanese government adapted a request-based approach for identifying projects. Now MOFA is responsive to the needs of low-income countries, but also emphasises their own responsibility for

\footnotetext{
${ }^{16}$ The Institute of Developing Economy was founded in 1960 as a statutory organization under the jurisdiction of the Ministry of International Trade and Industry (it is now the Ministry of Economy, Trade and Industry), acting as a social science institute for basic and comprehensive research activities in the area of economics, politics and social issues in developing countries and regions. Since the merger with Japan Export Trade Research Organization (JETRO) in July 1998, the institute aims at expansion of trade relations and promote economic Co-operation with all
} 
their own development. This strategy was motivated by the observation that low-income states do not always submit proposals which address global issues, such as poverty, basic human needs and the environment. As a result, MOFA created the Project Formulation Study Department within JICA, in order to identify more accurately the need for grant aid. However the proportion of projects proposed by this Department is small. This means that JICA's overseas offices only have a limited role and provide mainly adminiśtrative support to project processes, leaving official requests to be handled by the Japanese government in Tokyo. MOFA processes grant-aid requests and forwards other types of request, either to JICA or to the Overseas Economic Co-operation Fund (OECF). During the project design and implementation process, JICA makes frequent use of personnel from a range of ministries, including those who have no development experience or fluency in English (Beaudy-Someynsky and Cook, 1999).

The JICA brochure states, under the headline "Partners in sustainable development":

JICA is a governmental organisation which contributes to the economic and social development of developing countries and promotes international cooperation. Its activities range from long term technical Co-operation to emergency disaster relief. Established in 1974, Japan offers its services in a spirit of partnership to help fulfil Japan's global responsibilities. The keynote of its activities is the promotion of sustainable economic and social development, with special consideration for environmental issues (JICA brochure).

developing countries and regions, including Asia, Middle East, Africa, Latin America, Oceania, and East Europe(cited from http://www.ide.go.jp/English/Info/abut_ide.html) 
Japanese ODA: Partnership policy with NGOs

The Medium-term Policy, formulated by the Japanese Official Development Assistance (ODA) in 1999, is to implement a five-year plan based on the requirements of the government's and consultative body during 1998 and 1999. The plan will secure the understanding and trust of ODA at home and abroad, as well as ODA transparency, through effective and efficient implementation (MOFA, 1999a; 8-9). The Policy developers claim that its approach is primarily aimed at achieving the goals of the Development Partnership Strategy, as articulated in the DAC of OECD. The policy then emphasises the importance of "ownership" in developing countries and "partnerships" with aid donors. In particular, the Policy is committed to the formation of aid frameworks and measures to strengthen coordination between donor countries and international organisations, conditional on the self-help efforts and initiatives of the recipient countries (MOFA, 1999: 10-11). Issues of collaboration with other stakeholders are highlighted in the section on the mechanisms of aid. Key organisations are the Japanese Government, implementing agencies, the private sector, NGOs, other donor countries and international organisations.

In partnership with NGOs, Japanese ODA provides support both through financial co-operation and contracting services. According to Japan's NGO Center for International Co-operation (JANIC), it is estimated that in 1997 approximately 450 NGOs, including those with thirty years experience and those recently established, were working mainly to offer financial support to supplying goods and to sending field experts to support projects (Itou, 1998). Collaboration 
with NGOs is of particular importance in recent initiatives (MOFA, 1999). A grant assistance scheme for grassroots projects was launched in $1989^{17}$. The largest share of the funds goes to educational projects (39 percent of total amount disbursed 1998). These projects include the construction and repair of elementary school classrooms, followed by support for health and social infrastructure (MOFA, 1999: 24). Apart from grant assistance for grassroots projects, MOFA subsidies the NGO. This is designed to subsidise project costs, and accounts for less than half of the total project expense of Japanese NGOs in low-income states (MOFA, 1995). JICA's major NGO support scheme is the Community Empowerment Program (CEP), introduced in 1997. One case study research project is operated under this scheme.

JICA and MOFA recently increased their interaction with NGOs working in lowincome states in order to develop a dialogue and exchange views through regular meetings such as the "NGO-Ministry of Foreign Affairs Regular Consultation Meeting" and the "NGO-JICA Consultation Meeting" (MOFA, 1999). This highlights the importance of the stakeholders working together and committing themselves to international development assistance. Recent research by the Foundation for Advanced Studies on International Development (FASID) on the relationship between Japanese ODA and NGOs reported that both organisations have reservations about aspects of the above schemes. Criticisms include the fact that the presence of Japanese officers in the field is limited and short-term, and that this leads to the creation of the expression 'invisible aid.' Other

\footnotetext{
${ }^{17}$ It is handled by Japanese embassies and consulates to support non-profit organisations working at grassroots level including local and international NGOs as well as local governments.
} 
criticisms are of the fact that the vision of Japanese ODA, is unclear and that there is insufficient interaction with local counterparts (FASID, 1998).

\section{DFID and JICA}

One of the most significant differences between DFID and JICA, in terms of their organisational structures, is that JICA is an executive agency for Japanese ODA, and is operated under the aegis of the Ministry of Foreign Affairs (MOFA) whereas DFID remains an independent Department, at least for the present. JICA exercises much less autonomy in its implementation strategy than DFID. Since a number of government bodies intervene in Japanese ODA policy, decisions may be changed to reflect their competing interests. This imposes constraints on JICA's ability to promote development coherently. In contrast, decisions in DFID are reached by a single body of people with relevant professional expertise (Transcript 55). Since JICA is the agency responsible for development work, some held the view that it should also take responsibility through MOFA, for policy strategy (Transcript 58).

External consultants often implement DFID programmes whereas JICA uses internal experts, who require internal management within JICA (Transcript 55). This affects implementation. The Japanese ODA policy of using a request-based approach also makes its operations slow. This procedure, in which a large number of authorities are involved, is seen by its international counterparts, as inefficient and, according to USAID officers, "painfully slow" (Transcript 58). 
With regard to organisational relations with multilateral and bilateral donors, DFD and JICA have different networks. DFID has increased its contributions of official aid to multilateral organisations, mainly the World Bank and the European Commission, and has decreased its bilateral spending. Multilateral official aid grew from 197 million (US\$) in 1997 to 228 million in 1999 while official bilateral official aid decreased from 140 million to 98 million in 1999 (OECD 2001: 273). Japanese ODA is linked to the World Bank, the Asian Development Bank and the UNHCR, with which JICA has an official annual consultation (Transcript 58). As for bilateral relations, the bond between JICA and DFID has not been as close as JICA's relations with other bilaterals, as shown by one JICA officer's comments: "DFID is very far from us" (Transcript 58). JICA works more closely with USAD, the Canadian International Development Agency (CIDA) and Australian Aid (AUSAID) with which JICA has a staff exchange programme. Through this scheme, personnel from each organisation present their findings about JICA at the end of their stay, and this provides a vital learning opportunity for JICA. With DFID, there is nothing of this kind (Transcript 58). From my observation at international conferences in the UK, Northern European bilateral donor agencies, such as SIDA and German Technical Cooperation (GTZ) are more frequently mentioned than $\mathrm{JICA}^{18}$. It seems to me that bilateral to bilateral linkages emerge closely within the same geographical locale, perhaps because of their common international concerns.

${ }^{18}$ GTZ stands for in "Deutsche Gesellschaft für Technische Zusammenarbeit". 
The presence of JICA in the donor community in Europe is weak. It was observed by a JICA officer based in London that DFID initiates aid trends and strategies in London and brings its influence to bear on the international community. However JICA only learns of these policies and strategies at OECD meetings, by which time conclusions and issues have often been decided (Transcript 55). JICA may thus be seen to lag behind in this networking process. This difference in their strategic positioning is related to the capacities of the individuals in the two organisations and the extent to which they can act independently of their institutions. Often the initiative to influence aid policies is assumed informally by individuals, who use their informal networks rather than those of their institutions. JICA officers comment that individuals in other donor agencies can be autonomous in taking their agenda across agencies, whereas JICA officers are constrained by formal institutional representative roles (Transcript 55).

Differences in the organisational background of DFID and JICA are reflected in their experience of development work. The reasons for this include their organisational history and organisational culture. As far as the learning culture of the organisations is concerned, the confidence of DFID on the basis of its long established experience both in the field of development and its relations to Africa can have implications for its approach. The following citation from McGrath (2001) shows more clearly these concerns within DFD.

There are concerns that knowledge activities remain under-resourced and inadequately coordinated. There are also tensions over DFID's attitude towards knowledge sharing with external partners. Some staff are very bullish about DFID's role as a leader of development thinking 
and practice. One interviewee, for instance, spoke of the second White Paper as a "statement to the world". However, others were concerned about external perceptions of DFID as arrogant and worried that DFID appeared too certain about what worked in development (11).

McGrath (2001) further points out that within DFID, the official discourse on knowledge in low-income states is not strongly stressed. He comments that DFID staff note that the institutional approach to knowledge sharing is framed within a mindset of telling rather than listening. In direct contrast to the criticism of DFID's non-listening approach, Sawamura (2001) claims that the Japanese approach is characterised by a listening style, in his comparison of the origin of Japanese aid to those of other major donors. Orr's (1990) argument is used by Sawamura to stress that the western style of aid, influenced as it is by the experience of promoting Christianity, is based on "selling" an idea to the poor regions of the world. Sawamura (2001) argues that, in direct contrast to this, the fact that Japanese aid was initiated in conjunction with war reparations in Asia, partly explains Japanese restraint in transferring knowledge to poorer countries. He claims that in the case of aid to education, Japan adopts a position of wanting to learn both from poor countries and from its own experience. I assume that this argument can be supported by the fact that Japanese aid concentrates on school infrastructure and equipment supply, as well as on secondary science and mathematics education. Unlike aid to basic education, the fields in which Japanese aid tends to focus affect less directly the philosophy of nation building. This may explain why, as he claims, Japanese aid emphasises sharing Japanese experience rather than transferring knowledge in international co-operation. 
The above arguments from British and Japanese specialists on aid delivered by the two countries, imply different attitudes towards IOL and IORs during the processes of delivering aid. This difference is rooted in the origin of the aid as well as in their levels of confidence, cultures of reticence and degrees of experience. Overarching these differences is that fact that both DFID and JICA, inspired by the IMF, the World Bank and WTO, have committed themselves to programmes of international technical assistance for poverty alleviation that are also for market liberalisation in a global economy.

\section{Aid to Ghana}

The time has come to explain British and Japanese aid to Ghana. In terms of inter-national relations, the link between the UK and Ghana is much more longstanding than that that between Japan and Ghana. The British relationship with Ghana was developed during the colonial era and this is now seen by DFID as being an advantage in the overall British approach to Ghana:

The UK is well placed to assist, given our long and friendly relations, common language and systems. Our historic engagement has deepened our understanding of issues facing Ghana, shown that we can provide timely and quality advice and financial support, and so built trust and mutual respect. This provides us with an excellent opportunity to support and influence both government and other donors (DFID, 1998: 1)

In terms of ODA in 1999, Japanese ODA accounted for the largest amount of US $\$ 149.0$ million among DAC countries. After Japan, DFID provided US $\$ 64.6$ million (MOFA, 2000). Until the end of the 1960s, Sub-Saharan Africa had low priority in Japan's bilateral ODA (Inukai, 1993). In 1972, Africa received only 1 percent of Japan's ODA (Seddon and Sato, 1997) whereas it received 11 percent 
in 1998, in comparison Asia received 62.4 percent (MOFA, 1999). Ghana is the 22nd largest recipient of Japanese bilateral aid (based on cumulative net disbursements up to 1998) and the third largest in Africa. This also means that Japan is the largest aid donor to Ghana (as of 1997) (MOFA 1999a).

Proportionally speaking, UK aid to Ghana increased from 0.8 percent in the year between 1978 and 1979 to 2.3 percent in the year between 1998 and 1999. In 1999, the UK ODA to Sub-Saharan Africa was 47 percent of its total (OECD 2001b). The DFID Country Strategy Paper (CSP) for Ghana for the period 1998 to 2001 focused on supporting Ghanaian national development goals, which were set out in Vision $2020^{19}$. The strategy is that partnerships with government and civil society should be created and that all agencies should work together for development (DFID 2002: Part II). Ghana CSP objectives include supporting sector policies to provide better services, central reform agendas and to mainstream poverty strategy in all budgetary planning, as well as to create sound macro economic and fiscal management. DFID programmes are characterised by a close working relationship between DFID and the Ghanaian Ministries at Ministerial and official levels. In particular, DFID's support of a sector-wide approach (SWAP) to health has provided sustained funding to the sector, resulting in significant progress towards International Development Targets (DFID 2001). Over the period from 1998 to 2000, DFID has supported social development, especially in the areas of health and education (DFID, 2002). DFID has provided assistance to the Ghanaian education sector since 1987

\footnotetext{
19 Vision 2020 is a document published by the Ghanaian government on how Ghana aims to achieve middle-income country status by 2020 .
} 
mainly in the area of pre-service teacher education. In 1997, DFID started to help the Ministry and the Ghana Education Service (GES) to tackle institutional constraints and policy issues, to improve pupil learning and to develop the skills and performance of teachers (DFID/MOE, 1998). DFID continues to support Ghana's national policy of Free Compulsory Universal Basic Education (fCUBE), in which nine years of schooling for all Ghanaian children will be provided by 2005 (ibid.). In 1987, DFID supported a pilot stúdy for the National Functional Literacy Programme (NFLP) for adults, which was delivered by the Non-Formal Education Division (NFED) (MOE). A DFID senior education officer in London commented that Ghana receives one of the largest education programmes supported by DFID and that DFID sees it as one of the areas where they are pursuing international development targets. DFID's concerns in Ghana's education strategy are poverty alleviation and the development of public administration (Transcript 63).

Japan's ODA history in Africa is unique. Japan has neither political ambitions for, nor economic relationships with Sub-Saharan Africa. In terms of economic performance in the region, it is claimed that increased Japanese presence does not take the typical form of the trinity of aid, investment and trade. Inukai (1993) argues that:

the evolution of Japanese ODA to the region reflects not only economic concerns primarily associated with natural resources and export markets, but also the humanitarian concerns of the Japanese people and of a government policy backed by popular support" (261). 
This comment may be appropriate if a comparison were to be made with its aid to Asian countries, where the nature of trade and investment are explicitly revealed. However, considering the fact that Japanese aid practices in Africa are criticised as destroying natural resources, as pointed out by Sumi (1989), the comment maybe somewhat rhetorical.

As far as Ghana is concerned, three factors influence Japanese funding motivation. First, Ghana is a country of key importance in West Africa. It has been actively engaged in economic reforms since 1983. Ghana maintains good relations with Japan and as a country with a low per capita GNP (US\$390 in 1997), it needs a great deal of assistance (MOFA, 1999). Second, in providing ODA, Japan wishes to improve human resources, agricultural development, economic infrastructure, health and medical services, and environmental conservation (MOFA, 1999). Thirdly, Japanese aid to Ghana emphasises industrial development as a means of achieving sustainable growth, improving social services and enabling wider participation by the poor in the development process (JICA, 1995).

In relation to health sector support in Ghana, Japanese AID has concentrated on issues relating to population and HIV/ADS prevention. In the Global Issues Initiative (GII) on population and AIDS, Japan and the USA collaborate in selected areas. Japan contributed 3 billion US\$ to this programme between 1994 and 2000 in Ghana, one of 12 selected participatory countries in this programme (MOFA, n.d.). 


\section{Case study projects and their organisational contexts}

The DFID funded project was implemented under its Civil Society Challenge Fund (CSCF) from 1998 to 2001 . CSCF is open to any non-profit making organisation that shares DFID's overall objective of the eradication of poverty. It is based on matched funding and competition. Its purpose is not merely to tackle immediate poverty by delivering direct benefit, but also to confront the forces that keep the people in poverty by offering strategies (DFID, 1999).

The JICA funded project is operated under its Community Empowerment Program (CEP). The programme is categorised by its project-type technical cooperation. The program was created:

to directly benefit people at the grassroots level in developing countries for the improvement of their livelihood and welfare. Under this scheme, a model program, with endorsement of the recipient government, will be implemented by JICA together with NGOs, which play an important role in implementing projects at the grassroots level (JICA, 2000).

The invitation to apply for funds goes to programmes that use participatory approaches and local resources to provide direct benefit to the grassroots level. The programme supports community development for elderly people, the disabled, child welfare and health and hygiene improvement. This includes the empowerment of women and the improvement of living environments. The support also includes capacity-building and the promotion of local industries. JICA covers some of the expenses of the projects together with technical assistance providing Japanese experts (ibid.). 


\section{The Ghanaian Context}

This section gives an overview of Ghana, its current political and economic situation, its multi-cultural groups and commitment to social development. Adult literacy and primary health care are the areas of concern of the two case study projects. The implementing NGOs for JICA and UK funded case study projects, respectively, are introduced. Their relationships with government, other NGOs and community-based organisations are investigated in order to describe the environment of the case study.

Ghana first gained its independence from the UK in 1957. After a series of coups, a new constitution allowing for multiple political parties, was approved in 1992. Lt. Jerry Rawlings, head of state since 1981 , won the presidential elections in 1992 and 1996. In 2000, he was succeeded by John Kufor. In recent years, Ghana has gained a reputation of being one of the most politically stable countries in Africa.

As far as the economy is concerned, Ghana has twice the per capita output of the poorer countries in West Africa. Having said this, the country remains heavily dependent on international financial and technical assistance. Gold, timber, and cocoa production are major sources of foreign exchange. The domestic economy continues to revolve around subsistence agriculture, which accounts for 41 percent of $\mathrm{GDP}^{20}$ and employs 60 percent of the work force, mainly small landholders (The Republic of Ghana 2002). GDP growth is 4.0 percent per 
annum (World Bank, 2001). The estimated unemployment rate was 20 percent in 1997 (The Republic of Ghana, 2002).

As an aid receiver among Sub-Saharan nations, Ghana has been described as one of the most successful in implementing the structural adjustment programme recommended by the World Bank and IMF (JICA, 1995). Early in 1999, the World Bank launched the Comprehensive Development Framework as an initiative to promote greater coordination in development assistance within countries. Ghana was selected as one of the twelve countries to pilot this initiative and has used it to deepen coordination among its development partners in order to increase the impact of external resources on poverty reduction and in support of Vision 2020 (World Bank, 1999).

Although a steady improvement in human development in the country has been observed since its independence in 1957, its level of development remains quite low, ranking 129 out of 174 countries in 1998 in the Human Development Index $\left(\mathrm{HDI}^{21}\right)$. Ghana's HDI score is higher than those of Sub-Saharan African countries but lower than the average for low-income states. It is classified as having medium human development (GCCA, 1999).

Ghanaians belong to a large number of ethnic groups, approximately 100 ethnolinguistic groups such as Akan (Asante, Fante, Akuapem, Bono, Denkyira),

${ }^{20}$ Gross Domestic Product

${ }^{21} \mathrm{HDI}$ is an indicator of simple average of the life expectancy index, educational attainment index and the adjusted real GDP per capita index. It is calculated by dividing the sum of these three indices by three. It is widely used in international agencies (UNDP 1996). 
Dagomba, Ewe, Guan, Ga-Adangme, Wala, Builsa, Dagaaba. These may be further subdivided into numerous cultural and linguistic units. All these groupings have different dialects like Twi, Fanti, Asante, Nzema, Ga, Ewe, Dagbani, and Guan. English is the official language and is widely spoken throughout Ghana.

There are three main religious groups in Ghana: Traditional, 45 percent; Christian, 43 percent; and Moslem, 12 percent of the population. Churches of most major Christian denominations and mosques can be found in every region. To the people of Ghana, the traditions of their ancestors are an important part of their daily life. Traditional chiefs have historical authority over tribal and family matters. They are also custodians of land belonging to their respective clans or groups (The Republic of Ghana, 2002).

\section{Adult Basic Education}

Ghana's literacy rate is 79 percent for males and 61 percent for females ${ }^{22}$ (UNICEF 2000). These figures vary depending on the locations concerned. For instance, the literacy rates of the Dagombas who live in the Northern Ghana, with whom the DFID project was working, are estimated to be 3 percent, particularly in rural areas, according to the project field co-ordinator (Denteh, 1997). The main actors who deliver basic literacy programmes, other courses, and income generating activities, are the Institute of Adult Education of the

\footnotetext{
${ }^{22}$ According to Core Welfare Indicators Questionnaire Survey (CWIQ) 1997/98, which is based on a sample of 14,511 households, the adult literacy rate (above 15 years) in Ghana is 48.3 percent, with 40.4 percent in rural areas and 63.3 percent in urban areas. Gender disparities are 62.3 percent of adult males and 36.4 percent of adult females.
} 
University of Ghana and the Non-Formal Education Division (NFED) of the Ministry of Education. Other ministries also participate in an effort to eradicate illiteracy throughout the country (Boeh-Ocansey, 1994). The Ministry of Agriculture provides training for illiterate farmers and the Department of Social Welfare of the Ministry of Employment and Social Welfare provides vocational training.

In 1986, the Government of Ghana launched its commitment to increase literacy in the country, especially among women. This was linked to broad reforms in the education sector. The Non-Formal Education Division (NFED) was established within the Ministry of Education in 1987 to co-ordinate all non-formal educational programmes. NFED has delivered the National Functional Literacy Programme (NFLP) since 1991. The programme uses 15 Ghanaian languages to focus on adults of 15 to 45 years of age, mostly women. After DFID funded its two year pilot project, the World Bank provided US\$32 million over five years (starting in late 1999)(Ministry of Education, Ghana, 2000).

\section{Maternity and Child Health}

There are 19.7 million Ghanaians with 2.1 percent annual growth (World Bank, 2001). The Ghana Demographic and Health Survey (GDHS) suggests a declining trend in fertility and mortality in the period between 1993 and 1998, even though levels remained rather high (Population Council/PPAG, 2000). The total fertility rate dropped from 6.4 children to 4.6 for women in the 15 to 49 age group, with wide variations depending on place and education. Infant mortality 
dropped from 77 to 66 deaths per 1000, and child mortality from 155 to 119 deaths per 1000 birth. GDHS shows that in 1998 only 13 percent of married women were using modern contraception. Various organisations provide sexual and reproductive health services throughout the country, including the government, NGOs and the private sector. Although many are based in fixed clinics, community-based services have been increasingly introduced. The JICA project was working with the oldest and most developed NGO in the health sector. The NGO introduced community-based family planning for the first time in Ghana 25 years ago. The Ministry of Health, with funds from USAID and the Rockefeller Foundation and technical support from the Population Council, has expanded community-based advisory services and condom delivery throughout the country (Population Council/PPAG, 2000).

\section{GILLBT}

The Ghana Institute of Linguistics, Literacy and Bible Translation (GILLBT) is a non-government organisation, which started as a branch of the Summer Institute of Linguistics (SIL) International in 1962 to make God's Word come alive for people through Bible translation and literacy, in co-operation with the African Studies Department of the University of Ghana, Legon (GILLBT, 2000) ${ }^{23}$. The GILLBT mission statement reads as follows:

\footnotetext{
${ }^{23}$ The missionary work of SIL and its influence on indigenous culture is criticised. Some anthoropologists condemn SIL for destroying the traditional cultures and the social organisation of indigenous groups while others blame the missionaries for isolating the indigenous people and hindering their possibilities for development. The literacy campaign is SIL's means of cultural renewal that resulted in individualisation (as a consequence of Christian belief on the individuals' relationship to God), and secularisation (by giving scientific explanations for traditional beliefs were brought by missionaries) clashed with indigenous values and raised cultural inferiority among the indigenous. (e.g. Hvalkof and Aaby 1981).
} 
In obedience to the Great Commission, GILLBT exists to provide access to the Word of God in the mother tongue to language groups in Ghana and beyond, in partnership with others, through academic research, Bible translation, Scripture-in-use, literacy, development and training (GILLBT, 1995: 1).

SIL International has been working for over 50 years to study, develop and document the World's lesser-known languages, with its headquarters in Dallas, Texas, USA. It has carried out linguistic research in 1320 languages, spoken by 350 million people in more than 50 countries. SIL resources are primarily provided by affiliated organisations of Wycliffe International ${ }^{24}$. All SU workers are required to find funding for themselves and their projects (SIL, 2002) ${ }^{25}$. In 1980, GILLBT passed into Ghanaian management and expatriate SIL members were seconded to GILLBT. GILLBT is now affiliated to the University of Ghana. It is a large organisation with 185 full-time Ghanaian members and 33 expatriates, engaging with 31 local language groups in 6 regions of Ghana (see Appendix 4)(GILLBT, 2000; SI, 2000).

It is estimated that 60 indigenous languages are spoken in Ghana ${ }^{26}$. GILLBT has translated the New Testament into 14 languages and the complete Bible into two languages. Currently it is working on the translation of the New Testament in 13

\footnotetext{
24 "Wycliffe International's work is to facilitate the translation of God's Word into every language that needs it. Wycliffe has organizations in nearly forty countries and has had a part in translating over $500 \mathrm{New}$ Testaments into minority and indigenous languages. Fifteen hundred more translation projects representing over 70 countries, are in progress. Because Wycliffe organizations believe Bible translation is part of the Great Commission's mandate given to the Church, Wycliffe actively seeks to engage the world-wide Church in the Bible translation ministry" (Wycliffe, 2002).

${ }^{25}$ None of the expatriates working for GILLBT receives a salary from SIL. Churches and individuals in members' home countries, who are interested in their work, commit themselves to depositing a certain amount of money each month into their accounts. Individual members of SIL contribute 10 percent of the money they receive to support GLLBT's administrative costs (Transcript 15).

${ }^{26}$ Herbert and Robinson (1999) examine the multiple uses and purposes of diverse literacies and their impact on individuals and communities in the multilingual context of Northern Ghana, taking account of their personal, religious and economic implications.
} 
languages and the Old Testament in 12 languages (GILLBT 2000a). There are three separate departments in the operation: Literacy and Development, Linguistics and Translation. The project funded by DFID is managed by Literacy and Development, which incorporates material development, women in development, and income generation (SIL). The head office of GILLBT is located in Tamale, Northern Ghana. The GILLBT office in Accra plays a role in public relations and logistics needed by governmental offices and its partner organisations, such as the University of Ghana and relevant donors (See map in Appendix 4)

More recently, GILLBT has developed its partnerships on a wider scale within Ghana. They include "provision of consultancy services and participation and collaboration in field activities and training programmes" (Smith, 2000). GILLBT collaborates with academic institutions, such as the University of Ghana, which provide advice on GLLBT activities, and the Tamale Institute of Cross-Cultural Studies (TICCS), which provides training for new GILLBT staff. For technical collaboration, GILLBT's network includes local NGOs, such as Action Aid (Ghana), School for Life, SINAPI ABA Trust (SAT) and World Vision International. International NGOs such as Tearfund and the Jerusalem Trust earmark funds for GILLBT's missionary activities. In Ghana, GILLBT has worked for years with government bodies, mainly with the Non-formal Education Division (NFED) of the Ministry of Education. GILLBT has experience of working with bilateral organisations, such as CIDA and DFID on the basis of funded projects. GILLBT's funding sources vary depending on the 
activity. The major funding for Bible translation comes from US Wycliffe Bible Translation. However the funding for literacy work comes separately from organisations such as DFID, CIDA and Tearfund. As the director of GILLBT states:

We do have partnership and collaborating agreements, somehow are involved in literacy for example.... We exchange ideas, take part in their training, we help our methods of literacy, use our press to print books, ... complement each other, not competing each other (Transcript 8)

\section{PPAG}

The Planned Parenthood Association of Ghana (PPAG) started its advocacy role to promote family planning in Ghana in 1967, and was affiliated to the International Planned Parenthood Federation (IPPF) in 1968. The IPPF, operating at national, regional and international levels, is a federation composed of nationally based family planning associations (FPA). PPAG is the FPA in Ghana. Each FPA is autonomous and indigenous with its own culture and directed by local volunteers. There are at least two reasons for stressing autonomy of the FPA.

Firstly, as non-governmental bodies, FPAs are expected to be flexible and creative in their approaches to family planning. Secondly, and more importantly, because they are indigenous organisations, each FPA is expected to have greater credibility within its own national boundaries than would a Western-based international organisation... These volunteers, moreover, are usually drawn from the professional classes, with important connections to government decision-makers. Being volunteer-based, the local FPAs often gain people with both expertise and local connections, the employment of whom would otherwise have consumed scarce resources if their service had been 'purchased' by the Association. (Whitworth, 1994: 90).

The PPAG's organisational mission is described as follows: 
The Association shall provide couples and individuals with the knowledge and means of exercising their basic rights to decide freely and responsibly on their reproductive health as one of the means of improving the quality of life of Ghanaians. (PPAG, 1998: 1)

Its objectives are:

To assist national efforts aimed at improving the socio-economic and political life of the population; to promote the physical and mental health of families especially children, through better spacing of birth; to initiate and promote education and other programmes aimed at responsible family life for adults and youth (ibid.,: 1).

The association is the Ghana's leading NGO providing quality family planning services, education and advocacy in sexual and reproductive health and responsible parenthood. PPAG complements and supplements the efforts of government and other agencies, which provide sexual and reproductive health services (PPAG 1998). It aims to enhance family planning motivation by delivering relevant information to marginalized communities and to provide relevant information and services for youth. PPAG has pioneered: the community-based distribution (CBD) approach in Ghana to work with over 900 CBD agents; male involvement in family planning in Ghana; the integration of family planning into various income-generating activities for the benefit of rural women; a family-life education programme for the youth in Ghana, reaching 140,000 young people in 1996. It has also made inroads into rural areas through outreach programmes, with 70 percent of its clinics outside the regional capitals (PPAG, 1998).

The PPAG head office in Accra comprises the programme planning and technical support units of the research and evaluation unit, the service delivery 
unit, and the offices of youth and communication, gender and development. Finance and administration units are also located in Accra. PPAG implements its programmes in eight regions, divided into three zones, each headed by an Area Manager with support from regional programme co-ordinators (PPAG,1998: 16). (See Appendix 7 for location of PPAG activities)

Those organisations which work in partnerships with PPAG include international NGOs and other multi and bi-lateral organisations. They include, in particular, donor agencies such as IPPF, United States Agency for International Development (USAID), United Nations Population Fund (UNFPA), Japanese Postal Savings for International Voluntary aid (POSIVA), Japanese Organisation for International Co-operation in Family Planning (JOICFP), German Agency for Technical Co-operation (GTZ), Population Concern, World Bank, and Ghana Government (PPAG 1998a) ${ }^{27}$. In Ghana, PPAG works closely with the Ministry of Health, the University of Ghana and other relevant institutions.

GILLBT and PPAG are both Ghanaian NGOs. However, they are both affiliated to international bodies rooted in North America. Although their autonomy in operation is respected, it is difficult to say that they are independent of their parent bodies in programme strategy and implementation, given the nature of the funding linkages between them.

27 POSVIA is managed by Japanese government ministry. JOICFP is a Japanese NGO.
Population Concern is a UK NGO. 
Critiques of family planning, literacy and income-generating activities

Across the aid sector, small numbers of healthy, well-spaced children, literacy and income-generation are presented as unproblematic policies, linked to individual and household entitlements to livelihood and dignity. In fact, they have, over the decades, created heated controversy. What is now the Pro-life movement claims that population control may amount to the murder of the unborn child, through forced abortions, backed-up by coercive sterilisation and contraception (NRL, 2002). As an aid sector objective, population control and family planning are seen to be imposed by donor countries keen to protect their economies ensuring a continuing supply of the cheap natural resources from low income states on which they depend, of which large populations threaten to deprive them.

Contextual influences on literacy programmes are often questioned as well. In 1988, Jones found that multi-lateral sponsorship of functional literacy was affected by the organisational priorities of the funder and that the work-related application of basic skill development was difficult to realise in practice. Evaluation systems introduced by institutions, which provide literacy classes, were also found to be structured not only to measure the positive effect of learning on well-being, but for the convenience for the measurement tools. This was a criticism levelled at the UNESCO Experimental World Literacy Project in the 1960s and 1970s (Jones, 1988). Tension between knowledge gained at classes and oral modes of communication at the community level raises questions about the integration of indigenous culture with the technically rational 
culture being introduced through internationally sponsored programmes (Aikman, 1999).

Those doubtful of the politics of adult literacy and basic educational programmes oriented at marginal communities in low-income states have doubts. These reservations are magnified when literacy programmes serve as a vehicle to religious conversion, in the case of SIL and GILLBT to US-inspired evangelical Christianity.

Commitment to market deepening now sees income-generating activities as a prerequisite of community development projects, regardless of the particular activities they are promoting. For this reason, a significant proportion of the funding for the GILLBT literacy programme and the PPAG family planning work is to support income generation. The principle underlying most IGAs, is that small loans (in cash or kind) will be made available to community groups presenting a viable project proposal, with a business plan to ensure the sustainability of the activity and the repayment of the loan.

When IGAs are targeted at women they are criticised as being tantamount to an exploitation of women's labour (Simmons, 1997). For Simmons (1997), from the perspectives of economic development, women's productivity was being wasted since it flowed through informal channels, and remained unaccounted for and unexploited by the world market. Simmons argues that for the World Bank, women's productivity exists merely for the sake of its relevance to its market 
value. Integrating women into development for the Bank is thus, in effect, a means of channelling women's labour and produce through national and international business. Simmons further argues that "integrating women into development is approached by the international agencies purely from the dimension of increasing women's market-determined productivity" (246). She continues that

Income-generation projects are sometimes linked to suggestions for improvements in subsistence subordinated to the aim of raising women's market-determined productivity. These projects do not tackle the exploitation of women in export-process zones. They do not question the basic sexual division of labour or the international division of labour, in both of which women are placed at the bottom. ... There may be considerable advantages for women in having their own source of income, but this cannot be divorced from the social and political relations within which they must work. .... In these days, income-generation schemes can too easily reinforce oppression in the home and in the workforce (246-7).

Simmons stresses that some credit schemes do benefit women in helping them to establish an economic base. However, in many cases, women have no real control over the conditions of credit and production. Normally the administration of credit will be handled by the creditor, with women obliged to sell to a specific wholesaler at a fixed price, regardless of crop failures. Simmons says that "improving women's access to paid employment can smooth the way to further exploitation, poverty and social dislocation as well" (1997: 247). Population control programmes combined with income generating programmes are claimed as a 'tool for women to enhance their domestic power' based on the assumption that kinship structures prevent women from exercising choice over their fertility and that an independent income will enhance their capacity to do so (Jeffery and Jeffery 1998: 244). However as argued earlier, 
women's economic activity cannot be sufficient to make a difference to their influence inside the household unless women control the income to empower themselves (ibid.).

I have presented these critiques because there has to be some acknowledgement of the ways in which the activities sponsored by aid sector donors have been subject to as much criticism as that attracted by the polifical and economic motives of the aid system itself. Although this research is an analysis of contemporary project management and implementation processes, these cannot be separated from the principles and practices of the activities which they are concerned to develop.

\section{Managing partnership complexity?}

This chapter has introduced the bilateral organisations funding my case study projects and the NGOs responsible for their implementation It draws attention to the complexity of partnership arrangements when member organisations have different statuses, histories, purposes, structures, specialisation and networks. This kind of information, which is specific to each partner, will be taken into account in my investigation of communications between organisations and the learning that occurs between them.

Having learned about the different structures of each partnership and diversities of organisational members in this way, the time has come to discuss ways of gaining access to informants at the different field sites, and what this implies for 
the collection and analysis of data. This is the focus of the following chapter. It describes how I went about this work so as to allow me to report later in the thesis on the structure of relations and learning between partners to aid sector projects. 


\section{CHAPTER 4 THE RESEARCH JOURNEY}

\section{Introduction}

This chapter is an account of my research journey in which the methods used to collect and process my data are conceptualised. The methodology of the research and how the data was collected from multiple sources and means is described. The complexity of the research questions required detailed examination.

The processes of delivering community educational projects became important to me as a consequence of my experience of volunteer work with a Japanese NGO. Since then, I have pondered the question of why conflicts and friction between development agencies, including governmental funding bodies and local implementers, detract so markedly from the mutual efforts of providing collaborative assistance and why the voices of experience are not taken seriously in the construction of funding mechanisms. I became interested in understanding what factors influenced the processes of developing organisational relationships beyond the diversities of organisational culture. Having examined the complexity of funding mechanisms and processes of delivering projects in collaboration with actors, the importance of the impact of mutual learning within these schemes became apparent. 
These reflective elements of this research include techniques of autobiographical enquiry such as that described by Bell and Newby (1977) and Burgess (1990). Bell and Newby (1977) in Doing Sociological Research, encouraged authors to write themselves into their accounts; to address questions about how they did their research and the consequences the research has had for themselves personally. Burgess (1990) asked researchers to write a first person account reflecting on their experience of the interaction between theory and method in the studies they were doing. Now autobiographical reflection has become a critical element in understanding both the theoretical and technical aspects of the research processes (Burgess, 1990).

If one of the contexts of this enquiry is reflection on my own learning experiences, the dynamics of organisational relationships, both vertically and horizontally, are conceptualised in individual and organisational perceptions of partnership, as well as taking individuals' accounts of their experiences of learning. The investigation undertaken is a many layered analysis. It takes account of organisational cultures; their policies and capacities; the way they implement activities; individual accounts of work-related learning and personal capacity; the developments of intra- and inter-organisational relationships; interpersonal relationships; the processes of development projects; cross-national influence on organisations and individual social learning for well being.

Such complex inquiry has to focus the research protagonists in many ways. Comparative case study analysis has been suggested as one of the more suitable 
approaches. Case studies are concerned with the interactions of factors and events as practical instances, which can enable us to obtain a full picture of the interaction (Nisbet and Watt 1980) as well as identifying key issues which merit further investigation (Bell 1999). Ragin (1987) categorises comparative research into: case-oriented comparative methods and a variable-oriented approach. Bradshaw and Wallace (1999) indicate that typically case-based studies use only a few cases, which are selected for specified theoretical and/or substantive reasons. In accordance with this, my two case study projects were investigated to examine the themes associated with learning in partnerships. However, there is no intention to test general theories with a set of previously identified variables from various cases. Rather the study seeks to explore parameters of understanding relating to inter-organisational relationships and learning in the aid sector in a way that goes beyond work already undertaken in the business sector.

I investigate the research questions by comparing two bilaterally funded projects in one country in Africa, namely Ghana. Selecting a single country to host case study projects reduced the number of factors that need to be considered in the analysis. The value of conducting this kind of comparative research is corroborated by Bradshaw and Wallace (1991), who argue that the case study may be a preferred strategy in circumstances where a case partially supports the validity of a particular theory or set of theories. They claim that case studies have been used to point out the nuances of theory or the partial validity of theory and these studies also generate new beginnings in theory or at least new branches of existing theory. 
My research questions emerged as a result of the current debate on the increasing engagement of organisational collaboration. I also employ theories of organisational learning to examine how learning can impact on the processes and outcomes of organisational relationships. In this sense, using a case study approach may again be justified, in the sense that it enables the researcher to challenge the nuances of such theories in such a way that the complexity of aid mechanisms may be accommodated. Such an approach also aims to have implications for organisational learning theories in the aid sector where have both been yet recognised.

It is suggested by Huber and Van de Ven (1995) that organisational change can be studied either by focusing on a "variances theory explanation of the input factors that statistically explain variations in some outcome criteria", or "a process theory explanation of the temporal order in which a discrete set of events occurred, based on a story or historical narrative" (vii-viii). One might suggest that organisational learning, which consists of changes in organisations can also be studied in a similar way. It is important to stress, however, that the nature of research in this thesis is more suited to a process theory based on a story, allowing as it does for attention to be paid to the processes of individual learning which are rarely observed in statistical input or output measurements.

In the study of organisational change, a parallel analysis to facilitate, which interorganisational comparison, becomes necessary. Conducting parallel studies can 
"identify each organization's cultural and structural idiosyncrasies, as well as pinpoint their commonalities" and "cultural elements would cut across the use of all technologies at a given site and yet appear site specific when compared with practices" in other organisations (Barley, 1995: 7).

To ascertain how individuals conceptualise the experiences of partnership work and learning I used the narrative inquiry as the principal approach to collect data from my informants. Narrative data are obtained as stories from interviews, other texts and field notes based on the researcher's own observations (Lieblich et al., 1998). Lieblich et al. (1998) justify the narrative as a means of understanding human behaviour. They claim that verbal accounts and stories presented by individual narrators about their experience is one of the clearest channels for learning about a person's inner worlds. Josselson (1995: 32) justifies narrative as way of knowing thus:

Narrative is the means by which we... as researchers, shape our understandings and make sense of them. The questions is not whether narrative approaches are science,... the truths inherent in personal narrative issue from real positions in the world-passions, desires, ideals and conceptual systems that underlie life as lived...

Josselson (1995:33) goes on to describe the nature of the narrative approach and the way in which it contributes to the investigation that one is seeking for.

Narrative is the representation of process, of a self in conversation with itself and with its world over time. Narratives are not records of facts, of how things actually were, but of a meaning-making system that makes sense out of the chaotic mass of perceptions and experience of life.

My study sets out to explore such texts for what they reveal about the ways in which individuals build up relationships with people in their own organisations 
and in other organisations in their project partnerships. This is on the assumption that interactions will have implications for the role of learning. Individual accounts are drawn from selected events and experiences. Lieblich et al (1998) believe people's stories are usually constructed around a fact or life event, however they allow a wide margin of freedom for individuality and creativity in the selection of what they tell and this adds individual emphasis to and interpretation of these "remembered facts".

In analysing qualitative data and looking at the factors in data selection, it may be possible to structure an analysis of events using critical incidents technique (CIT). CIT was first devised in a scientific study by Flanagan (1954). Flanagan defines CIT as (1954: 327):

... a set of procedures for collecting direct observations of human behaviour in such a way as to facilitate their potential usefulness in solving practical problems and developing broad psychological principles...

Asking people to describe incidents which occurred over a specified period of time at work is one way of investigating the work people do (Flanagan, 1954). Carpenter claims that CIT relies on the idea that critical incidents will be memorable, making their capture possible either through interview, observation, or self-reporting. He stresses that "critical" implies a crucial effect on subsequent behaviour (Carpenter, 2000). Chell describes (1998: 56) the application of CIT to her interview method in organisational management research:

The critical interview technique is a qualitative interview procedure which facilitates the investigation of significant occurrences (events, 
incidents, processes or issues) identified by the respondent, the way they are managed, and the outcomes in terms of perceived effects. The objective is to gain an understanding of the incident from the perspective of the individual, taking into account cognitive, effective and behavioural elements.

Drawing on these principles, I planned to ask people to tell me about their observations of inter-organisational relationships based on their partnership experiences in the GILLBT and PPAG projects in Ghana. I sought to identify incidences of learning, and to select a small number of these critical incidents from the transcripts. I selected some conceptualisations of interpersonal and inter-organisational relations to develop the analysis of IOR and IOL which I describe in the following chapters.

\section{Exploring learning experiences}

The study relies heavily on the way in which individuals conceptualise the issues considered. It is concerned with ways of interpreting the meaning of that experience in relation to partnership and learning in and between various types of organisation. It would not be possible to talk to everyone in all the stakeholder organisations, nor would it be appropriate to use a survey to achieve a more representative picture of a sample of their individual members. In any case survey data would not capture the richness of unique individual accounts of important issues faced in daily life at work. Instead, this enquiry seeks information from individuals who reflect more or less holistically on the meaning of experiences they identify for themselves. 
As a narrative approach, life history methods can enable an effective analysis of how individuals account for and theorise about their actions and events in the social world, over time. The life history method can be applied to research into organisations as it enables the understanding of 'the ambiguities, uncertainties, and problematic which individuals experience and resolve on a daily basis' (Musson, 1998: 12). However I did not plan to use the life history approach to enquire into the individuals' entire background, but only to learn the processes and experiences of informants in relation to their work for the case study project and as appropriate, the organisation which employed them.

The conceptualisation of partnerships can be drawn, from written materials, but it has also to be taken from individual perceptions about inter-organisational relationships (IORs) which are most effectively obtained from interviews. As people talked, I was offered insights into how they saw problems between organisations in their project and whether the issues selected were related to relationships with partners, or to organisational systems and mechanisms. It also enabled me to sense what individuals found to be the most problematic matters in their lives as members of the organisations in question.

I used open-ended, in-depth and semi-structured interviews depending on the interviewee. Taylor and Bogdan (1998) claim that "the hallmark of in-depth qualitative interviewing is learning how people construct their realities how they view, define, and experience the world" (101). Open-ended questions were used and questions that can be answered by "yes" or "no" were avoided. It is because 
the open-ended nature of the approach allows the interviewee to answer "from their own frame of reference rather than from one structured by prearranged questions" (Bogdan and Biklen, 1998: 3). My aim was to hear how people construct the ways in which they work in collaboration with other organisations, and how they perceive policies of partnerships to be realised in their daily practice.

Two kinds of enquiry were made in semi-structured interviews: one, to obtain accounts of the interviewees' experience and the other, more focused, to seek for clarification about issues which were emerging as relevant to my study. These issues were mentioned by several interviewees or, already aroused from my own observations. Coffey and Atkinson (1996) refer to ways in which researchers describe the structure of narrative data. They identify four important elements which characterise how people tell stories in the way that they do: how they give shape to the events they recount; how they make a point; how they "package" the narrated events and their reactions to them, and how they articulate their narratives to the audience that hears them. The questions based on these four categories range from the generic to the more specific: "what was this about?" (abstract); "Who? What? When? Where?" (orientation); “ then what happened?" (complication); "so what?" (evaluation) and "what finally happened?" (result) (Coffey and Atkinson, 1996). Normally I planned to begin with general purpose of the research. For instance, I would say:

1. I am interested to know how your organisation became involved with the project and developed the relationships with your partner organisation(s)?; 
2. To what extent were you involved in this?;

3. How have you observed the way in which the relationship developed?;

4. In what ways do you think the project might have been improved?;

5. How does this kind of collaboration with organisation $\mathrm{Z}$ influence the management of the project?;

6. How do you see the differences from experiences you have had with other organisations?;

7. How do you interact with other organisations? On what occasion do you meet them?.

On occasions, to gain more information about an issue, I asked the interviewee to give me more detail. By listening to their stories, I hoped to conceptualise ways in which partnership learning might be constructed. I also encouraged them to talk about their personal learning experience by asking questions such as "What have you learned from this project at different stages?". I was often surprised when interviewees started telling stories of what had happened to them with the phase "I learned.....", since I had not used the word in my preamble.

One difficulty, which I encountered was to obtain stories and perceptions about partnerships from the perspective of the members of the recipient organisations, particularly their observations about their donor organisations. The beginning of my field research notes also illustrate comment on the struggles I experienced in obtaining critical data from Ghanaian interviewees:

Seeking for the story of IOL and changes, through interviews has been difficult. I also found that Ghanaians rarely express problems or make 
comments on negative aspects particularly about the issues related to donors. Expatriates (mainly British, American missionaries) have expressed their feelings more directly...... (Takahashi, fieldnote, Oct 2000).

I learned not to ask direct questions such as "Is the partnership arrangement going well?" or "Are there any difficulties or problems in working with your partner organisation?". Instead, I asked about concrete examples from their experience, bringing an issue of relevance into conversation, if it flowed well.

The differing responses which I received, may also have been caused by the biases of interviewees towards me as interviewer. Bradburn (1983) claims that interviewer characteristics which are clearly perceivable by respondents, such as sex and race, may influence the interview. Considering one aspect of Ghanaian culture, where the senior male is the most respected category, my appearance as a junior female might have been a disadvantage in terms of respect. This bias may also apply in British and Japanese culture to some extent. In addition, my Japanese appearance may have influenced the interviewees, for instance, people in the community were likely to perceive me as one of the Japanese donors and not as an independent researcher.

\section{Comparing projects}

The two projects chosen for study were funded by different governments. Ideally therefore, the case studies should provide a significant picture of collaboration between government as donors and NGOs as implementing partners. The projects selected were both in the area of social development. Each of them was 
being implemented in a similar local context and was at a similar stage of implementation, with similar objectives. One project was funded by the Japanese ODA with the involvement of local NGO and the other was funded by British development aid through DFID and implemented by a different local NGO.

Neuman (1997: 351) identifies three factors that are relevant to the selection of field sites: richness of data, unfamiliarity to the researcher, and suitability of area of concern. In accordance with Neuman's views, I selected projects offering rich and relevant data, bearing in mind the short duration of my fieldwork. Africa may be seen as the most suitable place for me because of its newness in relation to my previous experience and the suitability of language, i.e. English as a means of conducting my research.

\section{Field enquiry and subsequent analysis}

Gaining entry to case study organisations is a complicated process, as not all settings for field research are open to everyone and indeed, some require special permission for entry. Having said this, my specific point of entry into the field and the way in which further paths were opened up shaped my research enquiry.

Formal organisations (in this case, government organisations), invariably have formal gatekeepers who control access to a site (Bailey, 1996; Neuman, 1997). Entering a field site requires a flexible strategy, or plan of action for negotiating access and relationships with members. Dealing with gatekeepers is a problem 
which recurs each time a researcher enters a new level or area and this had an effect on the conduct of my research activities.

Several gatekeepers allowed me access to the projects and other people played the role of intermediary between the donor government and the Ghanaian NGO. Figure 6 shows the processes of my gaining access to the case study organisations at various stages. Regarding the JICA funded project, my first contact was with the JICA office in Tokyo (Fig. 6, no.1). They introduced me to the officer responsible for work in Ghana. He forwarded my request to JICA Ghana office in Accra for authorisation (Fig. 6, no. 2). Having waited for some time for a response, I eventually approached an acquaintance in JICA London for help (Fig. 6, 3-a). She made a call to the Ghana office and I received an email by return from JICA Ghana, explaining the delay in their response, and the high probability of acceptance of my proposal (Fig. 6, no. 3).

In the case of the DFID funded project, informal contact based on existing personal networks initiated my gaining access to SIL (UK)(Fig. 6, no. 1-a), who contacted its Ghanaian partner, the NGO, GLLBT (Fig. 6, no.3). My contact with DFID regarding the GILLBT project was made only after I completed my fieldwork, with the UK based desk-officer for the project. 


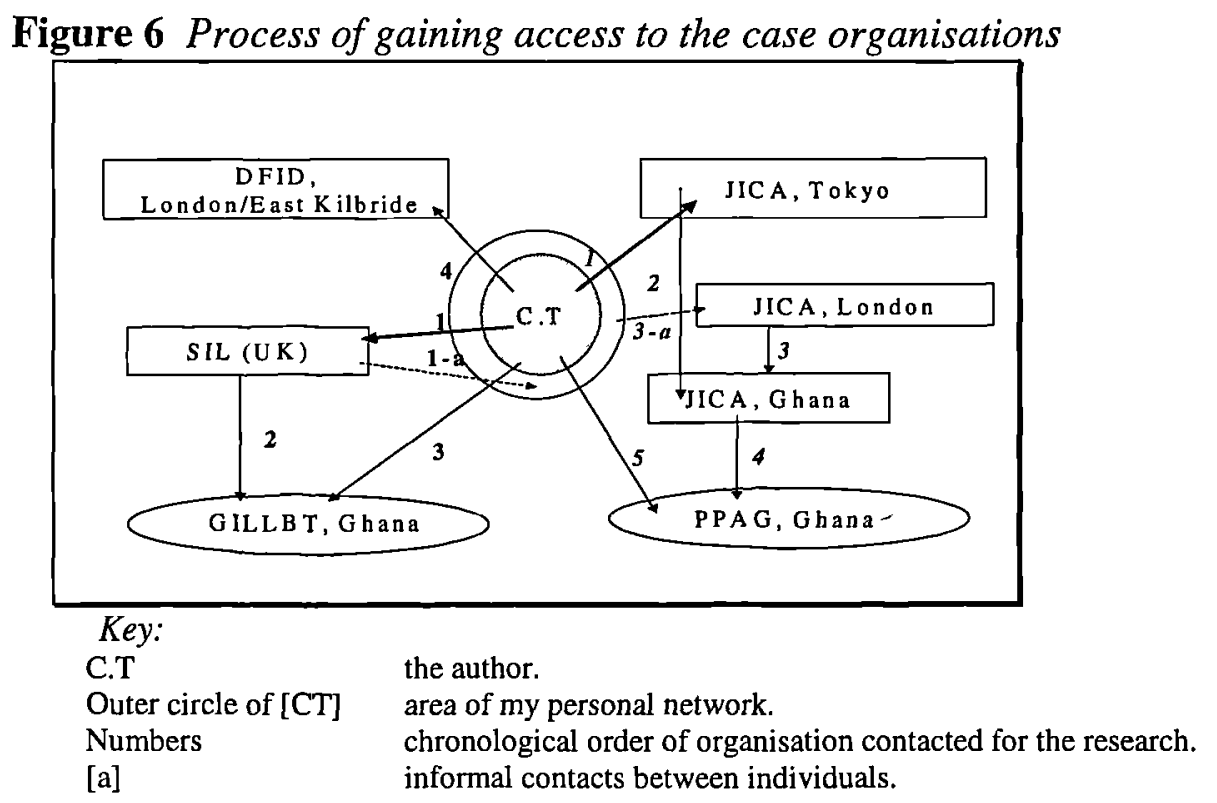

For contacts with local partners, JICA's Ghana field office and SIL (UK) played a major part in introducing and identifying staff.

The procedures for my introduction and access to the organisations were related to the hierarchical structures of the management of the project. With JICA, my initial contact was with the person at JICA headquarters; the JICA (Ghana) fieldofficer with approval by a representative of the field-office; an in-house consultant for CEP and the implementing NGO, PPAG. With the DFID case study, initial contact was with the NGO, SIL (UK) without consultation with DFID, and GILLBT was contacted by SIL (UK) directly.

Different organisational structures, in terms of operation, gave different kinds of access and the authorities had to be contacted in the right order. Indeed, the way that I accessed organisations and gained permission reflects the structure of the 
management of each organisation and the two projects. I was accessing the people according to the hierarchical structure of the flow of the funding in each project. My entry to the field, at different points of the project structures, matched the organisational hierarchy as well and had implications for the lines of enquiry available for further investigation. Also it enabled me to understand directly the structures of organisational relations and management of the two projects.

\section{Multiple lines of inquiry: management and implementation}

The methods used in this study are qualitative. They include document analysis, interviews and observation. The use of multiple sources of evidence in case studies provides a means of investigating a wide range of issues to show how levels of enquiry can converge (Yin, 1994). Multiple approaches to data collection help to reduce the bias of individuals, as well as of the researcher herself. Data collected were both primary, i.e. collected by the researcher at first hand, mainly through interviews and observation in this case, and secondary, i.e. available from other sources such as project reports. Since I found interviews to be the most interesting way of discovering information, data were collected largely from interviews. Documentary enquiry was the chief secondary source.

I interviewed a wide range of people in the different case study organisations including DFID, JICA, GILLBT, PPAG and other relevant organisations. For DFI, my informants included a desk officer for the GILLBT project, senior education advisers in London and Accra, and an officer of the British High 
Commission in Accra. Through interviews, I gained the perceptions from senior managers at SIL (UK) who were involved in the project and from members of GILLBT at different levels, including current and former directors, the associate director for the language programme, the literacy consultant and the funding officer. The staff in GILLBT included Ghanaians and British, American and Dutch expatriates. As GILLBT's collaborating organisations, Government officers and local NGO staff were also interviewed. They included the director and managers of the Non-Formal Education Division of the Ministry of Education, and field officers of Action Aid (Ghana).

My informants for the JICA project included officers at JICA Ghana field office who are responsible for the PPAG project in Ghana, those responsible for other schemes and senior managers. I interviewed JICA officers in London and Tokyo including the senior education adviser and officers at the Donor Co-ordination Division of the Planning and Evaluation Department, and the Africa Division. For PPAG, my informants were the director; the programme managers; the regional manager and the officer at the evaluation and research unit. As PPAG's major donor, I contacted staff at JOICFP in Tokyo to obtain their perceptions of the project. The people I interviewed assisted me in identifying issues in project implementation and organisational development relating to my enquiry. I then examined appropriate files and documentation. In this way, I was able to orient myself towards future interviews and observations. 
I gained information about the community users of the projects by observing their activities, as well as talking to their representatives. I observed literacy classes, literacy day activities to celebrate learning and the dedication of a completed Bible Translation in the GILLBT project. I visited outreach clinics for the PPAG project. Local members of both projects invited me to see the products made by women as income-generating activities. There I also talked to women about their stories in relation to the projects. In the GILLBT project, I interviewed facilitators and learners in literacy classes, and in the PPAG project, I interviewed a community-based advisor, volunteers and local co-ordinators about their work. Opportunities were available to gather information from village chiefs on their perceptions of the projects. The end-users, particularly women, contributed their stories of how their lives were being changed and their greatest difficulties in ensuring that they benefited from the projects.

I also elicited the views of people not involved with the projects about the relevance of the projects to social development in Ghana and their positioning in relation to foreign aid. These people included government officers and people from other organisations working in the field of adult literacy and primary health and researchers. Interviews were held with members of the government in Ghana, such as a member of the Ministry of Employment and Social Welfare, the Ministry of Health, and the Institute of Adult Education of University of Ghana, also members of NGOs, who were involved in community development. This helped me to gain an overview of the issues in the Ghanaian context, as well as 
to obtain a wide range of perceptions of the case study projects and organisations.

\section{The quality of the data}

In this section, the varying quality and quantity of collected data in two case studies are explained. The data collection processes were influenced by the environment of the two projects to the extent of my access and communication tools. The first reason for the variation in the amount and quality of data gathered foreach project relates to the physical environment and my location during the period of data collection. The most significant aspect of the physical environment in terms of my research was the distance between my residence and the research organisations. When I was researching GILLBT, I lived in the guesthouse, which GLLBT runs as one of their own income-generating activities. Not long after I settled, GLLBT moved their office to a backyard of their guesthouse. Despite my loss of one week when neither staff nor documents were available, the shift of their office not only saved me travelling time, it was very convenient for my research activities.

In the case of JICA, a friend of a professor in my Department at Warwick, accommodated me. Her house was located 20 minutes drive away by taxi from the JICA office building. Despite the time needed for travelling, this was still convenient, considering the size of Accra. However it took more than one hour to go to the two PPAG offices. This was because of the traffic and their location 
in a maze of small streets, which both the taxi drivers and I had to struggle to find every time.

The main difference in my experience of the two projects was that with the literacy projects I was based within the working environment of GILLBT, whereas with the maternity child health care project, I was neither in the JICA nor the PPAG offices. I soon felt part of the GILLBT organisation because I was able to decide when to visit the office and also the GILLBT staff were around all the time, during and after work. I was in a position which allowed me to observe and feel the organisational culture of GILLBT. In the case of JICA and PPAG, I was not in a situation where I could stay with them whenever I wished, nor could I observe their daily activities or feel the atmosphere of their work environment.

These differences in physical environment of the two case studies affected the quality and quantity of my data. Because of the length of time I spent with GILLBT, much more information was gained regarding internal issues and staff opinions of the donors. In contrast, the time I spent with PPAG was limited to a visit to the PPAG offices in Accra for an interview and a four-day visit to the project site. This fact should not necessarily be regarded as a drawback in using the comparative research method, since the issues and themes arising in each project are diverse, and even the same topics cannot be examined with exactly the same level of data, nor analysed to the same depth. 
Communication tools: interviews and language

I am a non-native speaker of English, using English as a communication tool for most of my research. The experience was quite different from using Japanese as my mother tongue when interviewing Japanese colleagues in JICA. How my use of English affected the process of collecting data is not easily measurable. My interviewees, with whom I spoke English, included both native speakers of English, including some from the UK and the USA, and some non-native English speakers, including other expatriates working in GILLBT, as well as Ghanaians. As a general principle, when interviewing in English, I taped the interviews. For Japanese interviewees, I used the tape recorder less often. The advantages of tape-recording were that this allowed me to capture the complete conversation (Taylor and Bogdan, 1998), as well as to concentrate my thoughts on the topic and dynamics of the interviews (Kvale, 1996). Tape-recording compensated to some extent for the disadvantage of using my English as foreign language, especially with informants for whom it was also a second language, a factor that might otherwise have led to some loss of comprehension during interviews.

I transcribed interviews, either from tapes or from notes written down during the interviews. I gave my informants the choice of being provided with the transcriptions and I handed over copies of transcriptions to those who required them. There were cases, in which the informants did not want transcriptions but only a report of my findings. I assume this was because reading transcriptions can be time consuming. On the other hand, a few interviewees requested me to correct and to add some supplementary information to the transcripts. I was also 
once advised by a British interviewee not to use direct quotations from transcriptions made on the basis of recorded interviews, due to my imperfect English and that of my informants. From the different interactions I had with my informants, I decided to present data by paraphrasing the content, for the purpose of providing information and clarifying events for most of the cases. However I also quoted some of the comments, which contained particularly strong messages.

My other concern related to collecting data from local participants with whom I could not use English as a communication language. In both the case of GILLBT and PPAG, regional co-ordinators who were fluent in English translated my questions and the local participants' answers. There were only a few cases in which I could communicate without translators. I had no way of judging the accuracy of their translation, particularly whether the answers I recorded were changed to represent the organisation more favourably. I was warned about using people who were involved in managing the projects as interpreters. However, this scenario was unavoidable since it was they who had brought me to the field.

It is important to be aware of some of the issues when the language used as a means of communication is a foreign/second language for members of one or both nationalities. Lisle (1985) suggests that language is not simply a medium for conveying concepts, it is part of the conceptual system, reflecting institutions, thought processes, values and ideology, and implying that the approach to a topic 
and the interpretation of it will differ, according to the language of expression (24-5 in Hantrais and Mangen, 1996: 7). English was used as the medium of communication during my field research in Ghana and in the UK. Difficulties in capturing every nuance, particularly with non-English speakers, were unavoidable. As Lisle claims, language should be carefully controlled, particularly in a study such as this, which involves various types of organisations and cultural backgrounds.

As a native speaker of Japanese, there was some advantage in my collecting data in Japan, as compared to others who may try to do similar research as nonJapanese speakers. For instance, I was able to access resources which are not translated into English, and I was able to benefit from the most recent Japanese debate by attending an academic conference in Kyoto. Being Japanese, even though not a member of the Japanese organisations, I may have been treated as an "insider" by interviewees and informants.

Being a Japanese speaker researching Japanese organisations may also present certain disadvantages. Japanese officers may be cautious in giving information, personally and institutionally, in ways not relevant to other foreign researchers. They may fear that I will exploit their information by criticising them in ways which it may not be possible for a foreigner to do. Japanese officers may also be cautious in what they say about other Japanese organisations or in saying what they really think of the organisations to which they belong. 


\section{Processing and conceptualising data}

After completing my fieldwork in Ghana, the next challenge was to categorise the data to discover its significance in relation to my inquiry. This was not fundamentally a mechanical or technical process, but a process of inductive reasoning, thinking, and theorising (Taylor and Bogdan, 1999: 140).

Figure 7 shows the overall processes of coding and analysing data. I first analysed the data into categories by theme and by concept (Neuman, 1997). I then tried to organise it broadly into: processes of individual learning; the nature of organisational relationships and the processes of change in organisations. 
Figure 7 Processes of overall data analysis

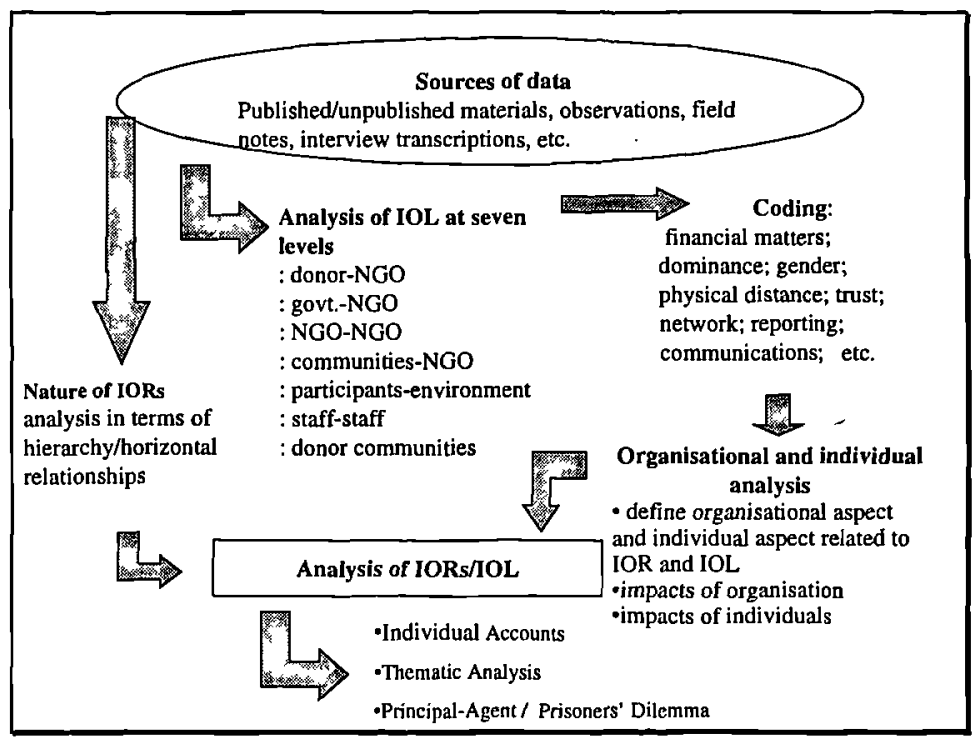

I used categories of different levels of IOR according to the hierarchy of each project (Fig. 7, Analysis of IOL at seven levels). The seven levels used were those between: the bilateral agency and the implementing NGO; the Government of Ghana and an NGO; NGOs and other collaborating local NGOs; participating communities and NGOs; participating women and members of their families; staff in organisations and in bilateral agencies. I coded the texts of the interview transcripts, field notes, and other materials to condense them and to bring to light common themes (Neuman 1997) (Fig. 7, Coding). In reading the texts for accounts of learning and intra- and inter-organisational relations, themes began to emerge as being felt strongly by most interviewees. I selected aspects such as issues related to finance, the nature of dominant relations, gender relations, effects of physical distance between organisations, issues relating to trust, the reporting requirements and the nature of communication. These themes were mentioned repeatedly by informants and they indicated the nature and 
mechanisms of IOR and their implications for IOR and IOL. Selecting these codes revealed points that required further investigation. Thereafter, I focused on the aspects of organisations and individuals which were associated with learning (Fig. 7, Organisation and individual). The main purpose of this was to analyse whether the linkage between relationships and learning was rooted in individuals or in organisations. Factors at the individual level included capacity, motivation, individual network and previous knowledge and experience. Organisational factors included any structural or external mechanism of an organisation or an organisational environment related to other organisations, which is beyond the influence of individual control. In the chapters that follow, I will present critical incidents which can shed light on some of these issues concerning learning and relationships in partnership arrangements. This will be done by introducing individual stories and by focusing on selected themes.

From the point of view of IOR, I examined hierarchical and horizontal structures, (Fig. 7, Nature of IORs) to draw out some implications as to how learning was facilitated and what prevented it with reference to the different structures of IORs. However I feel that such analysis was insufficient to portray the combined influence of inter-organisational learning (IOL) and inter-organisational relationships (IORs).

To explain the relationships between IOR and IOL, I tested the relevance of management theories against the relationships observed of the relationships. I applied two theories, mainly used in economics and management science, to the 
field of development. One was the principal-agent theory, applied by Killick (1998) to the relationships between donor and recipient in the conditionalities of structural adjustment programmes. The other was the two prisoners' dilemma of game theory, with which Smillie and Hailey (2001) describe NGO-NGO relationships (42). I decided to use the basic idea of these two theories in order to describe complicated structures and situations of IORs in the case studies. I then considered how learning might fit in with the structure-and argument of the theories, using narrative accounts from my data. I explained the relations between IORs and IOL using my data along with some theoretical ideas. The analysis of gender relations derived from women's accounts of experiences at the community level and the hierarchical organisational relations between community members also provided useful illustrations as to the issue of learning and daily life environments.

\section{My research journey: reflection on the inquiry}

Through my fieldwork in Ghana, Japan and the UK, my accounts of learning how to research, how to access data and how to communicate with informants reflects my analysis of processes and elements of inter-organisational relationships and inter-organisational learning. The systematic processes of the research journey appeared to parallel those of organisations working to build collaborative relationships with others. My account also suggests that my relations with informants mirror the accounts $\mathrm{I}$ was being given of how informants build their inter-personal relationship with members of partner organisations during the project cycle. Indeed, fieldwork by its nature requires 
the processes of interacting with people as noted by Devereux and Hoddinott (1992: 11) as follows.

The context of fieldwork reflects the conjunction of two sets of decisions about what lifestyle to adopt ('Living in the field') and how to interact with people encountered ('Living with others'). The personal and interpersonal dimensions of fieldwork can pose practical and ethical dilemmas on several levels.

In my case, my reflection on interacting with people throughout the different stages of my research journey seems to suggest its relevance to the issues that I was researching.

At the initial stage of my research journey, some of the difficulties and processes of identifying suitable case studies were similar to those experiences led by project funders in selecting partners. What matters for selection here were negotiations and compromises between initial objectives, the convenience of conditions and availability. Identifying and gaining access to potential projects for case studies of partner organisations in this study relied on the following capacities of the researched and the collaborators: the opportunities for linkage created by personal networks and implementation convenience in terms of time, means, and resources. The process of gaining collaboration both for research purposes and for implementing projects necessitates the same commitments and trust, which are shared mutually by those involved.

Several researchers of partnership practices in various fields have claimed that commitment and trust are the significant factors which lead organisations to build partnerships (e.g. Davis 1999; Bresnen and Marshall 2000; Sohal A. S et 
al., 1998). Commitment revealed by people at the top of an organisation is often regarded as the most effective skill, since it helps to guarantee commitment as a whole (Davis 1999), for instance, by being enthusiastic in learning about other organisations' activities (Sohal et al., 1998). I perceived this organisational effect in the role of the gatekeepers I encountered who, in many cases, were positioned at management levels. The gatekeepers played a significant role in committing staff within their organisations to assist me in my research, particularly through their interaction with senior staff. However on a number of occasions, it was difficult for me to judge whether it was appropriate for me to ask for more help or not. This dilemma may not exist in the scenarios of partnership arrangements, in which people commit themselves on the basis of contract or obligatory task. In this respect, I was aware of the fact that people offered me help as a favour, but that it was an additional task for them.

Hence, the development of my relations with informants, in terms of the degree of commitment, relied on how much I could make them feel interested in my research and make them willing to assist. Building trust-based relationships with my informants was required here. Establishing rapport with informants for researchers is very important for researchers (e.g. Taylor and Bogdan, 1998: 48). As an outsider, I had to build a relationship with each organisation during my period of data collection. Although my task as a researcher was not one of partnership, in a sense both I and the people in the organisations contributed certain inputs in order to achieve communal goals. It was necessary to make an effort to set up a good relationship with the members of organisations. As 
partnership based on mutual trust cannot be built in a day, it required a certain minimum duration. As I was looking for data that were based on people's experiences, I needed to make people trust me, so that they would talk to me in a relaxed, casual manner and share their experiences and opinions with me. Doing this required time, as well as a number of casual personal interactions not necessarily relating to my research topics. Also my personal behaviour and characteristics were observed since the exchange was two-way. In building this trust I was cautious of the limited time that I could spend with individual staff from each organisation. My mind was occupied with how I could obtain my data and follow the required social etiquette. My experience in building a relationship of trust within time constraints, appears to reflect the process of IORs in the case study.

In this chapter, I have reflected on the multiple methods used at different stages of my study. My account has integrated reflections on the different stages of my research process with references to relevant literature on method. In the final section of the chapter, I have discussed some of the implications of working between three countries for how I made sense of my data. 


\section{CHAPTER 5 ANALYSIS OF INTER- ORGANISATIONAL RELATIONSHIPS}

\section{Introduction}

Moving to my data, I describe the case studies themselves, with an emphasis on who is involved with whom and in what ways.

Information and perceptions were gathered from multiple different sources for each case organisation so as to given an insight from an array of perspectives into the development of the project. For instance, in the DFI funded project, email correspondence with a desk officer who managed the project provides detailed mechanisms of bidding procedures. A member of SIL was interviewed in order to clarify similar processes. For the JICA funded project, information was gathered from email correspondence with members of JOICEF in order to understand the background of the project. Brief descriptions of the activities involved in both projects were normally drawn from documentation and information relevant to the focus of the study was selected.

DFID funded adult literacy programme

DFID funded the Ghanaian NGO, GILLBT from September 1996 to September 1999 under the Civil Society Challenge Fund (CSCF)(replaced by the Joint Funding Scheme in 1999), through the INGO SIL (UK). SIL (UK) applied for 
funding on behalf of GILLBT and channelled funds to GILLBT. In total $£ 426$, 920 was disbursed over these three years. In CSCF, as the UK managing organisation, SIL (UK) submitted a project proposal to DFD Civil Society Department. The proposal was sent out to the appropriate DFID overseas office and/or the country desk officer in London for their comments. It also went to specialist advisers in DFD, in this case educational advisers. An independent adviser from the University of Edinburgh reviewed the proposal and commented on the sustainability of the project for funding. The desk officer managing the proposal in the Civil Society Department co-ordinated all the replies, following up queries from the appraisers before deciding on the overall suitability of the bid. This officer did not visit the project in the country of operation, but did have meetings with an SIL (UK) member to discuss financial and contractual issues. DFID monitored the project via a system of interim reports. SIL (UK) sent a project report to the desk officer, which was passed on to a specialist adviser who provided feedback (Transcript 1). At no point, was there direct contact about the project between staff at DFID and members of GILLBT. SL (UK) acted as an intermediary between DFID and GILLBT.

According to the project completion report written by an independent consultant to DFID (Smith, 2000), the prime activity of DFD in Ghana is its provision of financial support to promote adult literacy in 16 local languages, with the objective of improving lifestyles in Central and Northern parts of the country. The language projects funded are Adele, Anufo, Bassari, Birifor, Chumburung, Dagbani, Deg, Frafra, Gikyode, Lelemi, Mampruli, Nawuri, Nkonya, Ntrubo, 
Sisaala and Tampulma (Smith, 1999; see a map for their geographical location in Appendix 4). Activities sponsored emphasise community development through local language literacy classes, combined with Income Generating Activities (IGAs). Each language group enjoys different degrees of collaboration with local communities and project support organisations in respect of materials, facilitators, and approaches. The International NGO, Action Aid (Ghana) through which GILLBT uses REFLECT in one of its literacy projects is an example of this collaboration ${ }^{28}$. GILLBT and the Non-Formal Education Division of the Ministry of Education (NFED), together with other NGOs, have started the second phase of a nation-wide skill related adult literacy programme sponsored by the World Bank. At the time of my field visit in November 2000, GILLBT was preparing for the second phase of its project with DFID. Their application for second phase funding was at first rejected due to poor performance with the IGAs and for other reasons (Transcript 5). Following a recommendation from DFID, GLLBT has started collaborating with a specialist IGA organisation, the Sinapi Trust Aba (SAT). After making this change, DFID approved their application for a second round of funding. They received $£ 209,000$ as part of a three-year contract (SIL, 2002).

JICA funded maternity and child primary health care

JICA had funded a Ghanaian NGO, PPAG to introduce Integrated Family Planning, Nutrition and Parasite Control [Phase II (IP2)] in 10 rural communities. This was in the Eastern region under its new Community

\footnotetext{
${ }^{28}$ Regenerated Freirean Literacy through Empowering Community Techniques (details are in Chapter 6).
} 
Empowerment Programme (CEP). These 10 rural communities were located in the Amuana Praso and New Abirem sub-districts of the Birim North district. They were Old Abriem, Mamanso, Prasokuma, Noyem and Amuana Praso, Abokyikrom, Nyafoman, Nwinso, Abohema and Nkwateng (see the map of Ghana in Appendix 3). The Birim North district is among the three marginalized districts in the Eastern Region, in terms of health facilities and other socioeconomic infrastructure. At the time of my visit to the project in January 2001, the project which began in March 1998 and ended in March 2000, was at the final stage of a four year funding period (JICA/PPAG 1998). In total 5,175,000 yen (equivalent of $£ 27,178$ ) was disbursed during the funding years ${ }^{29}$. As the second phase of the Integrated Project (IP), the programme was being implemented in the Central Region of Ghana from 1987 with funds from IPPF and a Japanese NGO, the Japanese Organization for International Cooperation in Family Planning (JOICFP)(Transcript 61$)^{30}$. The plan for CEP was requested by MOFA/JICA (Tokyo) to JICA(Ghana). JICA (Ghana) identified the project for consideration because of its relevance to other Japanese ODA projects in the country. The proposal for the project was then submitted by PPAG to JICA (Tokyo) through JICA (Ghana). After an appraisal of the application by MOFA/JICA (Tokyo), channelled through the Embassy of Japan in Accra, the Minutes of the Meeting between JICA (Ghana), PPAG and the National Population Council of Government of Ghana were agreed (JICA 2001). The objectives of the project included: to increase the use of contraception; to improve the nutritional status of the children aged between $0-5$ years; to improve

\footnotetext{
${ }^{29}$ The exchange rate on the date of Nov. $16^{\text {th }} 2002$ was used.

${ }^{30}$ IP has been implemented in other countries in Africa, such as Tanzania. Like in IP in Ghana, IP
} 
maternal and child care services; to improve knowledge of sexual health and other reproductive health-related issues; to reduce the prevalence of intestinal parasites and to enhance community participation in and contribution to project activities. Like the GILLBT literacy project, the activities were to combine with IGAs.

The PPAG project emphasised community involvement at all levels in planning and management activities, include national and district staff and a local steering committee (See Appendix 5 for organisational structure of IP). Technical cooperation was contributed by the Japanese midwife who was based in the community for two years under the Japanese Overseas Volunteer Cooperation Program. The project gained particular attention from both JICA and PPAG because it was innovative. The project was seen by JICA as one of the most advanced and successful cases in Africa under the CEP, in terms of gaining an adequate degree of community participation. With respect to PPAG, an IP2 innovation was to extend the original model of project of IP1 to involve the District Assembly. This resulted in a greater degree of community participation than had been the case in IP1.

Figure 8 illustrates the patterns of organisational relations both hierarchically and horizontally in each project. This figure shows the existence of links, but does not indicate any thing about the quality of the relationships or the reasons for their development. Organisations shown within the ovals are Ghanaian and IPPF (JOICFP 2000). 
organisations. Lines with arrowheads at each end show linkages between organisations. Thicker lines between organisations refer to the specific IORs which are addressed later in the study.

Figure 8 IORs of case study projects

Figure 8a The DFID project

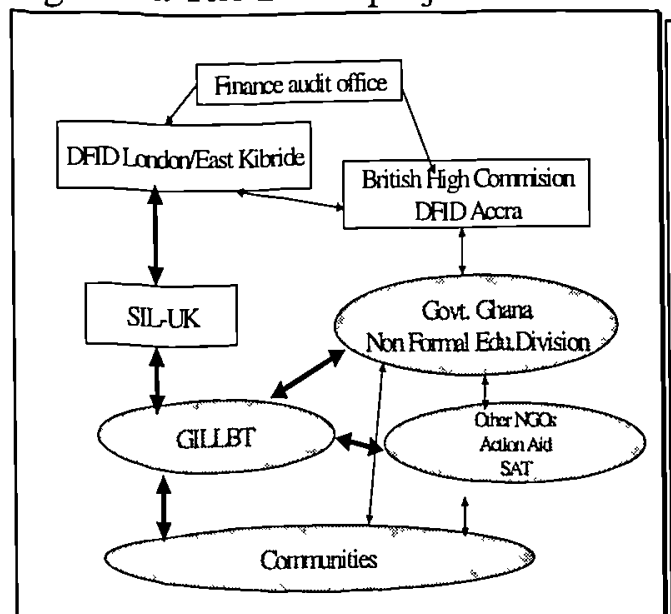

Figure $8 \mathrm{~b}$ The JICA project

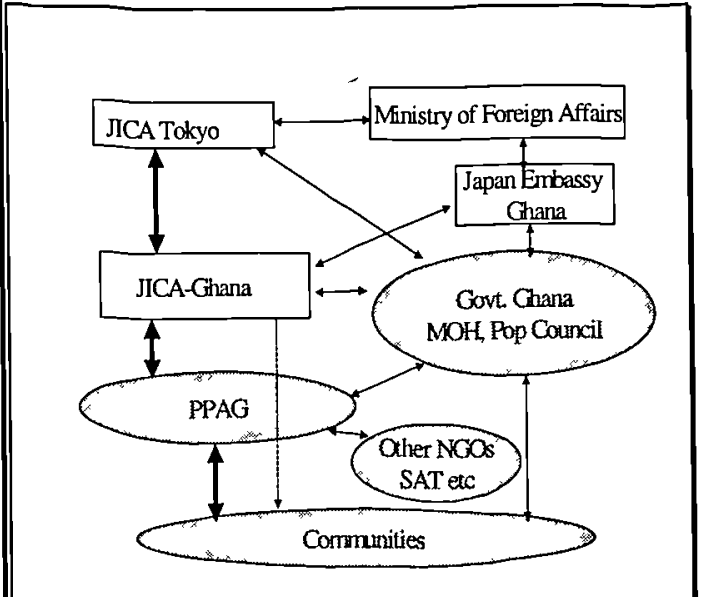

Key:

Ghanaian managing organisations. The staff may include both Ghanaians and non-
Ghanaians. e.g. GILLBT expatriate include British, American and Dutch.
UK or Japanese organisations
Selected linkages in the study
$\longleftrightarrow \quad$ Existed linkage, but not focused here.

The patterns of IORs explored in the case study include: the direct funding made by the bilateral government to the local NGO through an intermediary international NGO (DFID-GILLBT, via SIL-UK); the technical and financial support given by a bilateral government to a local NGO (JICA-PPAG via JICA Ghana); the technical co-ordination between a government and a local NGO (NFED-GILLBT); the financial and technical co-ordination between local NGOs (GILLBT-SAT); the financial and technical co-ordination between local NGO and international NGO (GILLBT-Action Aid Ghana). Stories told by informants 
allow an analysis of both present and past events, as they occurred at successive project implementation phases.

Table 3 identifies types of organisational roles and case study organisations in these categories.

Table 3 Actors in the project case studies

\begin{tabular}{|l|l|l|}
\hline Type of actor & \multicolumn{2}{|c|}{ Organisational actors } \\
\hline & DFID project & JICA proejct \\
\hline Funder & DFID & JICA \\
\hline INGO* & SIL & \\
\hline LNGO** & GILLBT & PPAG \\
\hline Government & NFED & MOH \\
\hline $\begin{array}{l}\text { Local } \\
\text { community }\end{array}$ & Regional office & District Assembly \\
\hline Other selected collaborators \\
\hline INGO & TearFund, & JOICFP \\
\hline LNGO & Action Aid (Ghana) & \\
\hline LNGO & SAT & SAT \\
\hline
\end{tabular}

$\begin{array}{ll}\text { DFID } & \begin{array}{l}\text { Department for International Development, UK } \\ \text { GILLBT }\end{array} \\ \text { Ghana Institute of Linguistics, Language and Bible Translations } \\ \text { JICA } & \begin{array}{l}\text { Japan International Cooperation Agency } \\ \text { Japanese organisation for international cooperation in family } \\ \text { planning }\end{array} \\ \text { MOH } & \text { Ministry of Health, Ghana } \\ \text { NFED } & \text { Non Formal Education Division, Ministry of Education, Ghana } \\ \text { PPAG } & \text { Planned Parenthood Association of Ghana } \\ \text { SAT } & \text { Sinapi Aba Trust, Ghanaian NGO specialised in micro credit } \\ \text { SIL } & \text { Summer Institute of Linguistics, UK }\end{array}$

\section{IORs and the parameters of partnership}

The present analysis explores the diversities of IORs in the case studies with reference to the parameters of IORs in partnerships. Table 4 shows a matrix of IORs in the case study projects, depicting the structures and patterns of IORs and some of the comments expressed about them. It also describes the stage of each project cycle at the time of my field observation. 
Table 4 IORs in two case study projects

\begin{tabular}{|c|c|c|c|c|c|c|c|}
\hline & & 1 & 2 & 3 & 4 & 5 & 6 \\
\hline & $\begin{array}{l}\text { General } \\
\text { Patterns of } \\
\text { IORs in the } \\
\text { aid system }\end{array}$ & $\begin{array}{l}\text { Partnership } \\
\text { involved }\end{array}$ & $\begin{array}{l}\text { Schemes/ } \\
\text { Motivation }\end{array}$ & Hierarchy & $\begin{array}{l}\text { Purpose of } \\
\text { relationship }\end{array}$ & $\begin{array}{l}\text { Quality of } \\
\text { relationships }\end{array}$ & $\begin{array}{l}\text { Stages of project } \\
\text { cycle } 10 / 2000- \\
01 / 2001\end{array}$ \\
\hline \multicolumn{8}{|c|}{ DFID FUNDED ADULT LITERACY PROJECT } \\
\hline $\mathbf{A}$ & $\begin{array}{l}\text { Bil'govt. \& } \\
\text { Inter' } \\
\text { NGO/local } \\
\text { NGO }\end{array}$ & $\begin{array}{l}\text { DFID, } \\
\text { SIL(UK) \& } \\
\text { GILLBT }\end{array}$ & $\begin{array}{l}\text { Civil Society } \\
\text { Challenge Fund }\end{array}$ & High hierarchy & $\begin{array}{l}\text { Direct funding to } \\
\text { local NGO, } \\
\text { through Int' NGO }\end{array}$ & $\begin{array}{l}\text { GILLBT doubt } \\
\text { about DFID not } \\
\text { trusting them. } \\
\text { DFID being } \\
\text { critical on outcome } \\
\text { IGA }\end{array}$ & $\begin{array}{l}\text { End of } 3 \text { yrs } \\
\text { funding. Process of } \\
\text { applying for the } 2^{\text {nd }} \\
\text { phase funding }\end{array}$ \\
\hline B & $\begin{array}{l}\text { Local NGO } \\
\& \text { Host } \\
\text { govt. }\end{array}$ & $\begin{array}{l}\text { GILLBT \& } \\
\text { NFED }\end{array}$ & $\begin{array}{l}2^{\text {nd }} \text { phase of } \\
\text { Adult literacy } \\
\text { campaign }\end{array}$ & $\begin{array}{l}\text { Medium } \\
\text { hierarchy }\end{array}$ & $\begin{array}{l}\text { Technical co- } \\
\text { operation }\end{array}$ & $\begin{array}{l}\text { Some GILLBT } \\
\text { staff hesitated to } \\
\text { collaborate due to } \\
\text { past experience }\end{array}$ & $\begin{array}{l}\text { Beginning of } 2^{\text {nd }} \\
\text { phase }\end{array}$ \\
\hline $\mathbf{C}$ & $\begin{array}{l}\text { Local } \\
\text { NGO\& } \\
\text { Inter' NGO }\end{array}$ & $\begin{array}{l}\text { GILLBT \& } \\
\text { Action Aid } \\
\text { (Ghana) }\end{array}$ & $\begin{array}{l}\text { Financial \& } \\
\text { Technical } \\
\text { exchange }\end{array}$ & $\begin{array}{l}\text { Low hierarchy } \\
\text { Competition }\end{array}$ & $\begin{array}{l}\text { Funding and } \\
\text { technical support }\end{array}$ & $\begin{array}{l}\text { Competition over } \\
\text { identity at } \\
\text { community and } \\
\text { diversities in } \\
\text { resource and } \\
\text { strategies }\end{array}$ & $\begin{array}{l}\text { Beginning stage of } \\
\text { collaboration in } \\
\text { marrying GILLBT } \\
\text { and AA approaches }\end{array}$ \\
\hline$\overline{\mathbf{D}}$ & $\begin{array}{l}\text { Local } \\
\text { NGO\& } \\
\text { Local NGO }\end{array}$ & $\begin{array}{l}\text { GILLBT \& } \\
\text { SAT }\end{array}$ & $\begin{array}{l}\text { Technical } \\
\text { support to IGA }\end{array}$ & Low hierarchy & $\begin{array}{l}\text { Contracting- } \\
\text { Out }\end{array}$ & $\begin{array}{l}\text { Slow in } \\
\text { proceeding } \\
\text { collaboration as } \\
\text { miscommunication } \\
\text { between them }\end{array}$ & $\begin{array}{l}\text { Beginning stage of } \\
\text { collaboration }\end{array}$ \\
\hline $\mathbf{E}$ & $\begin{array}{l}\text { Local NGO } \\
\& \\
\text { Community }\end{array}$ & $\begin{array}{l}\text { GILLBT \& } \\
\text { local } \\
\text { community } \\
\end{array}$ & $\begin{array}{l}\text { Adult literacy } \\
\text { classes and IGA }\end{array}$ & Low hierarchy & $\begin{array}{l}\text { GILLBT } \\
\text { delivering services }\end{array}$ & Various & Various \\
\hline \multicolumn{8}{|c|}{ JICA FUNDED MATERNITY AND CHILD HEALTH PROJECT } \\
\hline $\mathbf{A}$ & $\begin{array}{l}\text { Bil'govt. \& } \\
\text { Local NGO }\end{array}$ & $\begin{array}{l}\text { JICA } \\
\text { \&PPAG }\end{array}$ & $\begin{array}{l}\text { Community } \\
\text { Empowerment } \\
\text { Programme }\end{array}$ & High hierarchy & $\begin{array}{l}\text { Funding and } \\
\text { technical support }\end{array}$ & $\begin{array}{l}\text { PPAG felt JICA } \\
\text { was slow in } \\
\text { response. JICA } \\
\text { appreciating } \\
\text { organisational } \\
\text { capacity of PPAG } \\
\text { as partner } \\
\end{array}$ & $\begin{array}{l}\text { End of } 3^{\text {rd }} \text { year } \\
\text { funding. Process of } \\
\text { applying for } 2^{\text {nd }} \\
\text { phase funding }\end{array}$ \\
\hline B & $\begin{array}{l}\text { Local } \\
\text { NGO\& } \\
\text { Host } \\
\text { government }\end{array}$ & $\begin{array}{l}\text { PPAG } \\
\text { \&MOH } \\
\text { /Population } \\
\text { Council }\end{array}$ & $\begin{array}{l}\text { Various } \\
\text { interactions } \\
\text { (PPAG } \\
\text { involved in } \\
\text { policy strategy } \\
\text { advocacy etc) }\end{array}$ & Low hierarchy & $\begin{array}{l}\text { Mutual technical } \\
\text { \& Advisory } \\
\text { exchange and } \\
\text { collaboration }\end{array}$ & $\begin{array}{l}\text { Close and long } \\
\text { established } \\
\text { collaboration } \\
\text { PPAG being a } \\
\text { major NGO in } \\
\text { primary health. }\end{array}$ & Interview only. \\
\hline C & $\begin{array}{l}\text { Local } \\
\text { NGO\& } \\
\text { Inter' NGO }\end{array}$ & $\begin{array}{l}\text { PPAG \& } \\
\text { JOICFP }\end{array}$ & $\begin{array}{l}\text { JOICFP/IPPF } \\
\text { assisted PPAG } \\
\text { for IP } 1 \text {. } \\
\text { JOICEP helped } \\
\text { IP2 for the early } \\
\text { stage of JICA } \\
\text { funds }\end{array}$ & High hierarchy & $\begin{array}{l}\text { Funding and } \\
\text { technical support }\end{array}$ & $\begin{array}{l}\text { JOICFP recognise } \\
\text { IP as success story } \\
\text { in Africa in } \\
\text { obtaining } \\
\text { collaboration with } \\
\text { NGO and local } \\
\text { authority }\end{array}$ & Not observed \\
\hline$\overline{\mathbf{D}}$ & $\begin{array}{l}\text { Local } \\
\text { NGO\& } \\
\text { Local } \\
\text { NGO }^{32} \\
\end{array}$ & $\begin{array}{l}\text { PPAG and } \\
\text { various (e.g. } \\
\text { SAT) }\end{array}$ & Various & Various & Various & Various & Not observed \\
\hline$\overline{\mathbf{E}}$ & $\begin{array}{l}\text { Local NGO } \\
\& \\
\text { community }\end{array}$ & $\begin{array}{l}\text { PPAG and } \\
\text { District } \\
\text { Assembly } \\
\text { (local govt) }\end{array}$ & $\begin{array}{l}\text { District } \\
\text { Assembly } \\
\text { involved in } \\
\text { project } \\
\text { management. }\end{array}$ & $\begin{array}{l}\text { Low hierarchy } \\
\text { Traditionally } \\
\text { Competition }\end{array}$ & $\begin{array}{l}\text { Technical } \\
\text { collaboration }\end{array}$ & $\begin{array}{l}\text { DA contributes } \\
\text { cash to PPAG for } \\
\text { the first time in its } \\
\text { history. Close } \\
\text { collaboration is } \\
\text { established. }\end{array}$ & $\begin{array}{l}\text { Observed meeting } \\
\text { with PPAG and } \\
\text { District Assembly }\end{array}$ \\
\hline
\end{tabular}

${ }^{31}$ When JICA funding was delayed at the beginning of the project, JOICFP assisted financially to initiate IP2 (Transcript 61).

${ }^{32}$ The relation existed, however was not selected to explore in this study. 
The first and second columns refer to types of IORs along with the hierarchy of the aid system as shown IORs (A) bilateral government and international/local NGO; (B) local NGO and host government; (C) local NGO and international NGO; (D) local NGO and local NGO and (E) local NGO and community ${ }^{33}$. The rows give descriptions of IORs at various levels in order to have insights into the nature of the case study IORs. IORs are indicated for each case study in column (1), the motivation for them are shown in column (2), followed by an indication of their axis position on the project matrix (3). The purposes of the relationships are described in (4), so as to indicate how collaborating projects are implemented. In (5), I select the most forceful comments expressed about each organisation. Finally I indicate the stage of the project cycle reached at time of my investigation in (6).

From Table 4 above, it can be seen that IOR links reflect the partnership arrangements link, and that these represent various levels of hierarchy and different motivations of co-ordination. Since relations between funders and implementers are in the main hierarchical IOR, funders are in a position to control the activities of implementers. The latter have little role in decisions which have a significant effect on their own survival. This risks implementers losing their identity, given that they are dominated by funding bodies which set conditions relating both to financial matters and also to the content of the activities being developed. Compared to this, the relationships between the host government and local NGO, and those between local NGOs are less hierarchical,

\footnotetext{
${ }^{33}$ (C) an international NGO includes one which locally operates as a branch of international body. For instance, Action Aid is an international NGO based in the UK operating worldwide and as its branch,
} 
because the relationship between them is not primarily based on financial incentives. The motivation of such relationships is often to co-ordinate mutual technical efforts, in order for one organisation to enhance the activities of the other. This structurally horizontal relationship may become trapped in a competitive race to obtain scarce resources, identity and status. Whether IORs are set in a hierarchy or in a horizontal structure, relationships are characterised by the power of individual partners and their political interests. In this context, power is an advantage that one organisation has over the other, and politics is the organisational means of maintaining identity and territory while confronting outside experts in the delivery of aid. Organisations become involved in coordination activities with other organisations, both formally and informally. In either case, the development of IORs to achieve project goals may work beyond the organisational constraints of power and politics. Here, individuals and their networks, across organisational boundaries may complement the processes of day-to-day work.

\section{Inside stories: processes and development of IORs}

In this section, themes relating to characteristics of IORs in the case studies are examined by drawing on incidents at different stages of the project's life. The themes are explored on the basis of data obtained from my informants, captured in their interview transcripts and from documentary analysis. The section starts by describing the emergence of hierarchical and horizontal pattern of relations between organisations, whose existing networks are an important common 
feature at the initial stages. Accounts of the management and delivery of project activities reveal some of the processes through which organisations collaborate with each other. Experiences encountered in the two projects demonstrate the ways in which organisations build relationships of trust within organisational constraints. The processes involved extend from means of communications to an understanding of partner organisations. Diversity within their organisational cultures associated with staff, nationality, status and values are also influential in the understanding of these IORs.

Relationships are formed between partners, in pursuit of financial efficiency, technical support, and the reduction of workload. In the case of technical collaboration, diversities of organisational strategies and policy may lead to conflict. Here poor communication may interfere with the project implementation process, especially at early stages of the relationship. Other organisational characteristics, which affect project collaboration derive from individual experiences, but it seems clear that organisational regulations and structures are significant determinants of the nature and processes of the relationships that emerge.

\section{Identifying funding partners to start the work}

Existing networks are often regarded as the starting point of new IORs, because they do not require formal application procedure for membership. Several of the relations between organisations in the case studies demonstrate the significance 
of such networks for the creation of channels of funding in the first place and for the subsequent development of programmes. These networks were created on the basis of previous connections, including personal contacts and complementary organisational values. Among the criteria used in the process of identifying a partner, the most important are the organisational capacity of the proposed partner, its organisational reputation and mutually-shared organisational values. The process of identifying partners in diverse case study IORs reveal other factors, which may also be important in this early stage of project co-ordination.

In the case of the JICA project, informal and personal networks assisted in the selection of PPAG as a scheme worthy of JICA support, in part, because of PPAG's known organisational experience in the country. In the case of DFID, the implementing partner was identified through long-established formal procedures, but also had previous contact. When it comes to relations between local NGOs at a community level, the need for technical co-operation is the main motivation that lies behind the informal processes observed.

In the case of the JICA-funded project, from the point of view of PPAG, partnership choices were mainly driven by financial interests. This is consistent with the general experience of NGOs, particularly those dependent on external funds. From the JICA perspective, the process of selecting a partner was restricted not only by the capacity of possible implementing partners, but also by the capacity of JICA itself and the newness of NGO support scheme, which was 
at a rather immature stage at the time of this research. For funding purposes, the Japanese NGO, JOICFP, which had already funded the first phase of the project (IP1) introduced PPAG to JICA's new funding scheme and encouraged it to apply to extend practices of IP in new geographical areas. The JICA project formulation officer already knew about the activities of IP. He had also been acquainted with the staff of JOICFP, in his previous position elsewhere. This highlights how the linkage between JICA and PPAG for the purposes of funding was made through a Japanese NGO. Here, individual members of JICA and JOICEP members were crucial in building the partnership through a combination of protocols and networking (Transcript 26, 33 and 61).

From the point of view of JICA, the factors that determined the choice of PPAG as a partner were its organisational ability in terms of financial and organisational management capacity, and most importantly, its good reputation. This was confirmed by the recommendation from the staff of JOICFP. JICA's programme in Ghana had itself only recently been established and its regulatory mechanisms were not yet operational. Therefore, having a Ghanaian partner with rich experience of working with international donors helped JICA to implement its projects. JICA staff were certain that collaboration under this scheme would not have been possible if the partner had not been a capable and experienced organisation like PPAG. Members of JICA staff also stressed that those responsible in PPAG were very patient with their Japanese counterparts and willing to accept unexpected changes in strategy, requested by JICA 
(Transcription 26). It can be assumed that the PPAG staff helped the learning processes of the JICA staff, through the initial phases of their interactions.

\section{Negotiating NGO partnerships}

GILLBT's collaboration with SINAPI ABA TRUST (SAT) was initiated by a SIL (UK) member, who thought that GILLBT should increase its revenue through Income Generating Activities (IGAs), as recommended by $\mathrm{DFID}^{34}$. It assumed that poor management of the IGAs as affecting the loan recovery rate, together with the unpredictable market for the grain storage programme (GILLBT/SAT). The SL member suggested starting a partnership with SAT, an organisation specialising in micro-credit project support, because GILLBT lacked professional skills in this field ${ }^{35}$. He also observed that, since SAT and GILLBT were both Christian organisations, they could have confidence in linking up with each other, because of their shared Christian ethos. GLLBT's main concern was to make a link with organisations that would not exploit the people in the communities where they were working, nor their own members. These concerns followed from past experience of working in collaboration with other organisations.

In spite of common ideals, according to a GILLBT staff-member, the collaboration with SAT "has not been developed as much as we

\footnotetext{
${ }^{34}$ Information about GILLBT's collaboration with SAT was learned mainly from members of staff in GILLBT and not directly from SAT staff.

${ }^{35}$ In the collaboration, GILLBT sublet funds to SAT so that SAT could disburse the fund to participants of GILLBT literacy project at SAT's interest rate. SAT delivered the credit and award loans and collected loans. GILLBT had no liability for loans taken by borrowers. Both SAT and GILLBT monitored the process to ensure that the participants repay loans on time (GILLBT/SAT)
} 
hoped"(Transcript 12). It took longer than expected to start the formal collaboration, after agreeing on roles and responsibilities. About 8 months before the interview which I conducted with a GILLBT member, GILLBT staff went to observe the SAT micro-credit project-training programme with proposals from GILLBT for how SAT might support their work. Having visited the GILLBT projects, SAT staff were to set up an account for the transfer of funds from GILLBT to SAT. However, after about 8 months nothing had been done. The SAT response to the GILLBT request was slow, leading one of the GILLBT staff to express doubts about the outcome of the collaboration. This shows how delays and breaks in communication can sour the level of trust between staff who are trying to establish collaborative relations between organisations, regardless of how busy they are.

The problem was eventually solved, when an SIL UK staff member visited SAT. This intermediary found that each side had been waiting for the other to initiate contact and to play the lead coordinating role (Transcript 12). This confirmed the lack of communication skill between the different actors, which adversely affected the implementation process and gave a negative impression of each to the other as a prospective partner. This example shows how identifying a certain partner, using a shared ethos as a positive indicator, may not necessarily enhance the quality of communication. Rather, failure to recognise the limitations of professional management skills seems to have been the most significant issue. 
During the process of making a memorandum of understanding (MOU) between GILLBT and SAT, there was further disagreement this time over the sharing of any profit between the two organisations. The MOU states " all the fees (application, training and process) and interest income shall constitute profit and will be shared in the ratio of 70:30 percent for SAT and GLLBT respectively ${ }^{36,}$ (GILLBT/SAT:3). In negotiation, GIILBT suggested that the division should be equal at 50:50 percent each. SAT insisted on the ratio of 70:30 percent, justifying this with the claim that it took large amounts of time and expense for them to travel to different IGA development sites. The SAT position was accepted, even though GLLBT had raised the possibility of having one member of SAT staff working full-time at the GILLBT project's expense. Another difference arose in relation to SAT was GILLBT's organisational purposes. Each community IGA participant has to pay a joining fee, which would be about 3 percent of the micro credit loan. GILLBT would advance the fee to participants because they were unlikely to be able to pay for themselves. However SAT saw this money as part of the community's profit. SAT and GILLBT had a different attitude towards the IGAs. In the eyes of GILLBT staff, despite the fact that SAT is an NGO, it acts as a commercial bank, whose priority is its own profit and not the profit of participants. This approach was alien to GILLBT philosophy, since GILLBT is a humanitarian missionary organisation without knowledge of micro-credit. In the event, this experience with SAT was an opportunity for GILLBT to learn how to increase income from community IGAs, so as to sustain its own activities with less dependence on donors. For

\footnotetext{
${ }^{36}$ For instance, SAT would train and equip GILLBT staff with micro-finance skills for a fee.
} SAT would also provide the orientation and training associated with credit management, again 
GILLBT, the main purpose of collaborating with SAT was to assist the IGAs of the literacy programme grant. They were said to be critical to the survival of communities where money was increasingly scarce. One of GILLBT's regional language group co-ordinators expressed his own eagerness to work independently of GILBT and thought that the income generated from local resources through the micro credit schemes would allow their own activities to stabilise and avoid future dependence and troubles with donors (Transcription 16).

The GILLBT/SAT collaboration aimed to reduce the workload of GILLBT staff, particularly their regional co-ordinators. As Cairnes who did an evaluation on a GILLBT programme on behalf of CIDA observes:

Transfer of the revolving funds to SINAPI management would relieve GILLBT personnel of a task for which they are not necessarily well qualified. IGPs [IGAs] are an important way to generate funding for the overall program, and to assist socio/economic development (2000:17).

Above all, learning from an experienced, specialised not for profit organisation such as SAT builds GILLBT's organisational capacity and saves a large amount of time and expense. Without DFID criticism of the poor performance of GILLBT's IGAs, GILLBT might not have taken action to collaborate with SAT. It might indeed have repeated the mistakes of several years ago, made by other organisations. A local NGO consultant comments that the need for NGOs to share experience has increased, particularly in technical areas such as IGA management (Transcript 49).

for a fee. 
This section has highlighted ways in which organisational ethos may influence the operation of partner collaboration, creating obstacles with resulting structures and systems. They include a lack of flexibility in the rules and regulations that govern schemes and bureaucratic organisational management systems. Organisational operations, such as the means of communication and characteristics of staff, also influence the quality of IORs. A mutual understanding of partners' organisational environments becomes crucial for effective project operations.

\section{Inflexible implementation}

The rigidity of organisational management style seems to have been a factor felt particularly by several actors in the two case studies, as a constraint on the process of working with other organisations. Several informants expressed their frustration at the inflexibility of the system in which they were working. This problem existed not only between organisations, but also within organisations.

Funding system requirements are one of the principal determinants of daily procedures of development project work. Flexibility in the system of financial disbursement and associated duties is therefore important. Implementing agencies, have little opportunity to be involved in the decision-making processes affecting their own funding management procedures. The frustration of not being able to influence financial mechanisms was also observed by members of funding agency staff based in overseas field offices. They have to be obedient to head quarters, when dealing with issues of finance with local counterparts. 
It was often said that JICA's management style made the implementation of activities very slow. PPAG staff commented on the number of delays, particularly delays of disbursement, due to JICA bureaucracy. Staff working in the JICA field office in Accra had to obtain permission from their counterparts in Tokyo before doing anything. They felt it all took too long. In time this had a negative effect on the relationship between JICA (Ghana) and the local NGO in terms of mutual trust. The following quotation drawn from a PPAG report reveals the local effects of this in the project implementation.

Generally the delay in the release of funds has some negative effect on the project though PPAG provided some funds to undertake most of the preparatory activities, community enthusiasm was so high that, most members have been left in suspense because more of the major activities have not been organised (PPAG 1998)

..... For instance, the renovation of the Amuana Praso Health Centre could not be carried out as planned. Collection of stool samples and deworming exercise were also affected by the delay (PPAG 1999:10).

In the first year, it took three months after the project started for PPAG to receive the first payment, due to required processes of approval by JICA headquarters in Tokyo. In the second year, disbursement was delayed for two and a half months because JICA waited for an audit, before starting the next year's funding. At the start of the third year, based on these previous experiences, JICA (Ghana) discussed with the Tokyo office the possibility of starting the next year's programme without waiting for the audit. The delay was shortened to two months. Although these delays in disbursement did not affect the daily activities significantly, it caused the postponement of workshops and other programme activities. An in-house consultant for CEP commented on the importance of 
making payments on time, in order to maintain a good relationship with local NGOs and others involved (Transcript 27).

PPAG also felt that report deadline requirements and the need to collect information for them put unreasonable pressure on people (Transcript 34). The fact that the field office has less authority to make decisions on cases that require particularly prompt action, limits management capability and inhibits the promotion of a good working relationships with local counterparts in the field. PPAG senior staff claimed that there were no differences in individual capacities within the various organisations. They saw the constraints in collaboration as being created by the system rather than by the individual staff competency (Transcript 34).

Frustration at disbursement delays was also observed in JICA's Ghana office. Based in Accra, it was excluded from the financial decision-making processes in Tokyo. The experience of the JICA Ghana office provides an example of the field office struggling with the bureaucracy of its own head quarters and with local requirements. Effective implementation depends on timely payments, especially in areas where limited cash is available. A senior JICA adviser commented that the Japanese system of accounting is a significant constraint on individual project funding schemes. These events are dependent on Japanese parliamentary decisions, which are far beyond the control of JICA, even its senior headquarters staff (Transcript 60). 
Another example of the restrictions created by Japan's bureaucratic inflexibility was revealed in the restraint felt by a programme co-ordinator of PPAG, who attempted to amend the field operational plan of a project, to take account of the realities of the situation in the local community. He complained that there was no flexibility to modify a proposal even if, at the moment of implementation, it was no longer relevant in the field (Transcript 36).

\section{Mid-term reporting requirements}

Project rules and regulations may lead directly to oppressive relationships between partner organisations. Funding agencies' requirements, imposed on implementing organisations, are the mechanisms through which funding agencies maintain their control. For instance, fulfilling reporting requirements is one of the main concerns of implementing organisation managers. They have to pay attention to this in order to make and maintain a good impression about their organisation's capacity and its activities. However, implementing organisations often find donor demands too difficult to meet, in terms of the level of statistical detail required relating to activities, such as number of literacy learners, dropouts and, above all, about financial transactions (Transcript 11$)^{37}$. Moreover, there are additional demands for organisations receiving funding from several sources and having to meet their different donors' reporting requirements. Reporting requirements for the same project may have formats, concerns and areas of interest to be emphasised in reports vary with the donors. For instance, each PPAG donor, has its own expectations of the programme's development

\footnotetext{
${ }^{37}$ In remote areas with a limited culture of book keeping when even paper may be scare, the
} 
process. USAID is interested, in the final analysis, in the rate at which the use of contraceptives is increasing. The UK NGO, Population Concern, wants to be informed about how the issues identified in its youth programme relate to how they use contraception and the extent to which young people are involved. The donors interested in community-based programmes, e.g. JOICFP and JICA want information, not only about contraceptive use, but also about parasite control and income-generating activities, in order to monitor more holistic community development (Transcript 33).

Donors' expectations of reports are largely based on their own reasons for providing the funding. For instance, Christian NGOs are interested in knowing how the activities benefit communities in terms of the Christian perspectives, as well as in respect of the impact of literacy programmes. In contrast, reports for government cannot mention evangelical activities, as religious aspects of programmes lie beyond the scope of their interest (Takahashi, Fieldnote 2000). As for financial disbursement, it is much more complicated to audit a project when each funded project starts at a different time, with different reporting requirements (Transcript 10). It was also pointed out by members of the local NGO that all donors want full recognition of the contribution they have made to be stated clearly and publicly at all opportunities, and they may be reluctant to support projects, which have finance from other sources. This means that a local NGO has to make a clear line of demarcation between the activities supported by different donors, even for projects promoting the same activities in the same areas. They have to take care not to mix their contributions and not to cross 
budgets (Transcript 33). This implies another kind of pressure from donors, which may not be clearly stated anywhere, which implementing organisations need to address. The pressure here is determined by the self-interest of donors.

\section{Knowing each other at distance}

As understanding others is crucial to the development and maintenance of good human relationships, organisations should have positive insights into their partners' operations. The issue was raised as a result of one partner failing to understand another, and of a lack of basic reliable information about each other's environment and organisational structure. For instance, a number of my interviewees, based in Ghana, discussed the problem of getting their sponsors to understand the implications of the physical environment, in which they were working. This is the case particularly where communication was not based on face-to-face interactions, but occurred at a distance, using email, fax, letters and telephone.

Communication is a mode of inter-personal behaviour. However, the physical conditions which determine the quality of inter-personal communications seem to be one of the central issues influencing the quality of IORs. That there is a contrast in terms of quality of mutual understanding between donors who have a field office in Ghana and those who do not was mentioned several times. The differences included the degree of understanding of the situation faced by the local NGO, and the technical inconvenience in corresponding and interacting in 
real time. The difficulty of dealing with a donor who does not have a field office was expressed as follows.

... but difficulties I see (not having a person in the country) is that there are so many things that you have to explain because they are not in the country. It is difficult to appreciate some of the issues (Transcript 33).

For instance, living in a situation where the value of the currency can change dramatically every week, can help a donor to understand how changes in a budget which was designed six months before might be unavoidable. This is difficult to understand for a person who has always lived in a county where prices are stable (Transcript 33). Having visited the remote areas where a project is being implemented and having lived in the country for a while, the donor representative will be more aware of the difficulties, for example of transportation and of collecting accurate statistics in rural areas (Transcript 15 and 33). This means that donors who have experience of living in the field might not require such information at very short notice. Having a residential office can be helpful for understanding the situation of one's counterpart. The donor becomes aware of the fact that certain things should not be demanded because they are just not feasible in the field, even though they might be normal requests in the country where the donor's headquarters is based. There may be reasons why some donors do not have a residential office in-country, but there are consequences of this, which will affect the quality of the relationship with local implementing NGOs. Having said that, the differences in understanding of local contexts may not have great effect on those who manage a financial audit either at headquarters or in the field. 
Such inter-organisational differences may not only relate to a lack of donors' understanding of the local context. There may also be a case for donors and NGOs to build mutual understanding, through face-to-face interaction. NGO staff stressed the differences in the quality of relationships with donor representatives whom they had met in person and those with whom they had had no direct communication (Transcript 15). This seems to be one of the drawbacks of relying on the developments in information technology as the principal mode of communication, which reduce the opportunity for face-to-face communication.

\section{Obstacles to effective partner relationships}

Comprehending how organisations are positioned in relation to partner organisations requires careful attention. This was felt to be an essential part of the knowledge process through which understanding of a partner's organisational behaviour can be enhanced. This is particularly significant in the case of an understanding of the nature of structures in funding relationships between donors and recipients.

For a number of staff in GILLBT, DFID was seen to require more detailed information on budgets than other donors, and GILLBT found this difficult to manage. (Transcript 10, 11 and 12). According to a member of SIL (UK), this impression derived from GILLBT's misperception of DFID. This in turn was a direct result of the lack of information GILLBT staff had been given about DFID's organisational environment. This impression also arose because 
GILLBT staff did not perceive that their relationships established with other donors were associated with differences in the different funding mechanisms they used. This same member of SIL (UK) claimed that DFID was not particularly hard on GILLBT, but that DFID staff were forced to be firm by their own national audit office. There was an incident in the past, he recounted, in which DFID called GILLBT in Accra at very short notice for an explanation of their financial reports. This coincided with a time that the GILLBT accountant was on leave and GILLBT was not able to give the required explanation to DFID about details of the account. This left DFID with an unsatisfactory impression of the meeting. For GILLBT, it left an impression that DFID did not trust GILLBT's reporting mechanisms. The SIL (UK) staff officer explained that the mission to audit the GILLBT programme did not come from DFID, but rather, from the UK national audit office, which selected Ghana at random, in an overall audit of the Joint Funding Scheme (since replaced by CSCF). Even the DFID desk officer at East Kilbride, in charge of the project, was only informed about the journey of the auditor from London to Ghana, when the mission group was already on the plane (Transcript 5).

GILLBT staff felt that DFID requirements were stringent, when compared to other donors such as CIDA. There was a quite different arrangement between DFID and GILLBT and CIDA and GILLBT. In terms of financial reporting, the member of SIL (UK) understood that CIDA could provide a grant for LEAD Canada through more informal means than is the case with other NGOs working 
with $\mathrm{CIDA}^{38}$. He also claimed that the Wycliffe SIL system was a high-trust environment, in terms of expenditure, so that full accountability details were not an overt requirement. This was attributable to the common Christian ethos of the organisations. In contrast, DFID's accountability requirements were stringent. GILLBT was forced to conform fully to DFID budgeting procedures. This had been a very hard lesson for both expatriate and national staff working at GILLBT (Transcription 5).

PPAG staff showed their understanding of how JICA's Ghana office works in relation to JICA headquarters. When they were asked about the differences between JOICEP as an NGO and JICA as a government agency, they explained that JOICFP was more flexible in the management of funds. When PPAG sent their proposal to JOIFCP the decision was made there and then, whereas JICA took time before taking a decision, because of the bureaucratic processed binding JICA Tokyo (Transcript 34). Although PPAG found that JICA took a lot of time in responding and dealing with various matters, especially in relation to finance, they showed their understanding that this was due to JICA's management mechanisms and not the inefficiency of individual JICA staff members.

JICA may pressurise partners to provide reports by a certain date and information within a certain time-scale. As far as financial resources are concerned, JICA had invested more in the IP2, than JOICFP had contributed to the IP1 phase. This brought with it both disadvantages and advantages (Transcript 34). For individual staff members, there was no difference, since the constraints were

\footnotetext{
${ }^{38}$ GILLBT received grant from CIDA channelled through LEAD Canada, Canadian NGO.
} 
within the system rather than in individual ability to do the work (Transcript 34). Indeed, understanding of what partner organisations were expected to do in relation to their accountable parties helped to explain the reasons for confusion and the apparently unsatisfactory management style of partner organisations.

The DFID-GILLBT relationship via SIL (UK) also seemed to create confusion for GILLBT staff, regarding who was accountable to-whom, and who was responsible for what. This was a result of not understanding fully the structure of these inter-organisational relationships. One of the GILLBT staff members wondered whether the detailed information required was for SIL (UK) or DFID (Transcript 12). Apparently there was no direct contact between GILLBT and DFID (UK), but only indirect contact through SIL (UK). It was recognised by some staff that SIL (UK) may have added some requirements of the partnership based on points raised by DFID. This triangular relationship caused complexity and communication problems which prevented each organisation from fully understanding the other. For example, most of the reports to DFID were written by GILLBT and sent to SIL (UK). SL (UK) then modified them and submitted them to DFID. According to staff interviewed at GILLBT, the relationship between SIL (UK) and DFD was not well understood by GILLBT. This seemed to be one of the elements causing GILLBT confusion, about the role of SIL (UK). It also implied doubts about the relationship and who has more control. This may have been sorted out at the time when SIL (UK) explained to GILLBT the mechanisms of DFID funding and its own accountability to its national audit office, but had occurred before my interview with GILLBT took place in Ghana. 
Another member of staff also explained that there were both positive and negative effects of having SI (UK) as an intermediary actor, between GILLBT and DFD. She said that

In some ways, this can cause some difficulties because SIL has to know exactly the way we are thinking and the way we are working on the field, which demands a great deal of communication. Sometimes a phone line is down and we cannot communicate by email. It is very expensive to communicate on the phone (Transcript 11).

On the other hand, the advantage is that SIL (UK) can contact DFW directly and sort out matters face-to-face (Transcript 11).

\section{Organisational culture}

The characteristics and processes of IORs may also be influenced by a sense of uniqueness which each organisation possesses. Each organisation is different from others, just as no individual is the same as another. The organisations in the case study projects vary in a number of aspects, some of which were associated with their individual organisational cultures. The differences between them is reflected in their organisational status, whether it is governmental, nongovernmental, religious or voluntary. The effects of the national and local culture on organisational culture and their effects on the processes of IORs may be as important as status, particularly in cross-national comparative research such as this across three countries. This section focuses on features of organisational culture which have contributed to the analysis of IORs in the case studies. It also questions whether the differences observed in organisational management styles 
affecting the quality of IORs derive primarily from differences in organisational culture.

\section{National differences}

Most of the interviewees in my case studies commented on the scale of differences in the management and funding of projects by people of different nationalities. The ways in which organisations relate to each other can, of course, vary with the different nationalities of their staff, and with characteristics of the county in which their organisation is located. However this variability seems to be also due to the nature and history of organisational linkages, rather than to the immediate effect of national differences. Nonetheless, comments were made about nationality. For instance, NGO staff stated that they knew that the Japanese were interested in the minute detail of activities (Transcript 33).

The fact that GILLBT is an organisation with both national and expatriate staff representing several nationalities has positive and negative effects on the relationships between staff and on ways of learning from each other. GLLBT runs an orientation programme, both for Ghanaians and expatriates, aimed at explaining these differences in cultures and values (Davies 1996). However expatriates still expressed the need for training on-the-job in order to understand Ghanaian practices, such as the opportunities of using of organisational facilities for private purposes (Transcript 15). One of the expatriates expressed the value of having Ghanaian secretaries, which helped to avoid misunderstanding about issues such as these (Transcript 15). 
Some interviewees commented on the differences in attitude between Ghanians and expatriates towards certain issues. Several expatriates even mentioned that problems arising from change in organisational management tended to come from expatriates, rather than from the Ghanaians (Transcript 5 and 15). As compared to Ghanaians, expatriates seemed more resistant to change. Not only should people of different nationalities develop good inter-personal and interorganisational relations, but Ghanaians from different regions encounter similar problems, in terms of human relationships and communications, as the following quotation illustrates.

Meaning what we say is based on our background. Even among Ghanaians, the one from the North and one from the South can have differences (Transcript 13).

However, it is treated differently from miscommunication between Ghanaian and expatriate groups.

Organisational differences: NGOs and governmental organisations.

From the point of view of NGOs, as funding agencies, international NGOs are seen more friendly in approach and flexible in respect of funding than the bilateral government organisations, to the extent that this has emerged as a stereotyped characteristic of NGOs. One GILLBT expatriate who used to work as a missionary in a local community observed that the relationships with bilateral aid organisations were like those in a business, whereas the relations with NGOs were sympathetic (Transcript 7). For instance, within NGOs, there was a strong tradition of exchanging Christmas cards, and asking about the 
progress of projects. Yet for her, differences between NGOs and bilateral organisations were also found in the strategies operating behind their funding. For instance, DFID took the stance of expecting people to be able to sustain activities on completion of a funding period, so they were likely to cut off funding even if the project was not working as well as expected. In contrast, UK NGO, Tearfund, through which GILLBT was partially funded, was said to be more sympathetic to continuous support. She commented that this as an effective aspect of a trusting relationship.

\section{Evangelical organisations}

In the case of GILLBT, its organisational background as a Christian NGO and the fact of having been funded by an evangelical organisation for most of its existence had created a fundamental difference in funding relationships. SIL (UK) staff assumed that GILLBT's working with Wycliffe SIL did not require much accountability in terms of expenditure and financial reporting, due to the trusting relationships between Christians. However, this attitude of GILLBT had to change in the case of relationships with secular governmental organisations, like DFID. Learning how to obtain funding from GILLBT's new partner agencies was very hard for GILLBT (Transcript 5). Some GILLBT staff also felt that the reason for DFID's strict accounting was the suspicion that GILLBT might have tried to mix funding for literacy work with that of its Christian mission work (Takahashi, field note, 2000). It seemed that for GILLBT having to make a clear-cut budgetary distinction between literacy work and Bible 
translation was a difficult challenge, since they had never had to do this when they received funds from other Christian organisations.

\section{Personal capacity and linkages}

Personal linkages may be seen to work in both negative and positive ways. Since it is individuals who establish the bridge between organisations, the ways in which organisations relate to each other rely on individual relationships. Personal linkages are a crucial bridge between organisations, and their value extends from serving as an entry point for IORs as a key element in enhancing the implementation phase of a project cycle. A positive statement came from a PPAG member of staff who conceded that the closeness of JICA involvement in the early stage of the project had benefited the project co-ordination throughout. It meant that JICA staff understood what PPAG was aiming to do, and the concepts and approaches that were used. This facilitated a better understanding between organisations (Transcript 34). It was made possible by the fact that JICA has an office in Ghana.

On the other side, some of GILLBT's relationships with other organisations were coloured by negative inter personal relationships between members of their staff. It may have been perceived, for instance, that the relationship between NFED and GILLBT also suffered from negative personal links. However the relation between NFED and Action Aid seemed to be more positive. This leads on to the issues of implementing projects in collaboration with others, considering the characteristics of the relationships between them. 


\section{Realities of partnership}

Firstly, the nature of the relationship between donor and recipient in the aid sector may not be as clear-cut as is the case in the business sector. For instance, an NGO member who had previously been employed as an accountant in the private sector claimed that in donor-NGO relationships, donors had their own goals and purposes which determine how their money is spent. NGOs have to adjust their aims in order to get funding, which results in their losing their identity in the process of implementation. In businesses, organisations decide where to allocate money for themselves for profit, but in the donor-recipient case, "donors dictate to the recipient on how to use money" (Transcript 20).

PPAG often struggled with community expectations and their perceptions of the project as an opportunity to bring 'something' to the community. The community expected PPAG to restrict its involvement to giving money, but not to get involved in community decisions that would affect their lives. Therefore, PPAG had to make an effort to make the people in the communities understand that projects were meant for themselves, a process that took almost three years. This PPAG experience is similar to JICA's experiences in the community.

Such perceptions of asymmetric power relationships colour the whole picture of inter-organisational relationships in the aid sector. The achievement of goals through mutual inputs from those working together in partnership is rarely observed. The structures of IORs remain markedly unequal and organisations must either accept asymmetric power relations in order to achieve their own 
goals or challenge their structural constraints in order to increase their mutual benefits. Such organisational choices and the process of co-ordinating hierarchical relationships are examined in Chapter 6.

\section{Partnerships difficulties at the community level}

When NGOs collaborate as partners, conflicts may arise from differences in organisational strategies, their interests and technical and financial capacities. As distinct from donor-recipient relations, collaboration between NGOs working in the same communities is not hierarchical, but rather horizontal and competitive in nature. Differences between such NGOs become the trigger for making relations between them competitive. The extent to which organisational capacity and approach are based on policies, strategies and experience, shapes interorganisational structures at this level. In the case of GILLBT and Action Aid (Ghana), the rich international backing Action Aid (UK) and the long-term local commitment of GILLBT were based on different kinds of knowledge. This caused difficulty when each organisation sought to benefit differently from their collaborative arrangement. In attempting to marry their different approaches, conflicts arose because each party insisted on maintaining its own identity. In addition, there were problems associated with organisational structures. These related variously to resources, status, policies and objectives.

Similar patterns of stories were observed between GILLBT and NFED. In the first phase of the national functional literacy programme initiated by NFED, the gap of understanding between policy-makers at a national level and those 
implementing the project in the field might have explained the unsatisfactory outcomes. In the event there was a lack of consultation at the regional and local levels, little was discussed with community-based organisations in terms of technical approaches and agreement on sharing resources. In addition, GILLBT staff felt that NFED staff, taking advantage of their status as members of a government body with generous financial support from the World Bank, were exploiting GLLBT's local resources including their facilitators and the information they held about learners (Transcript 16). It has been remarked that NFED staff were too busy to collect data in order to produce reports for the World Bank. Instead NFED went to the communities, where GILLBT literacy classes were already established, collected their data and used it in their own reports (Transcript 46).

As far as the relations between GILLBT and Action Aid (Ghana) and between GILLBT and NFED are concerned, there was an issue of which partner was the dominant one in each case. Relations between them were not as obvious as the donor-recipient relationship in terms of a pre-existing hierarchy, associated with financial control. Instead, collaboration between them was characterised by obscure power relationships and complex arrangements for the management of shared resources. In this way, the differences in their organisational structures seem to have created complex modes of collaboration. Table 5 illustrates the diversity of partnerships in practice and lists some examples that contrast the rhetoric and reality of IORS processes. 
Table 5 A contrast between rhetoric and reality of IORs

\begin{tabular}{|c|c|c|}
\hline $\begin{array}{l}\text { Formation and } \\
\text { Process of IORS }\end{array}$ & $\begin{array}{l}\text { What is claimed (rhetoric)? } \\
\text { What are the forms? }\end{array}$ & $\begin{array}{l}\text { What happens (reality)? } \\
\text { What really matters? }\end{array}$ \\
\hline (a) Patterns & $\begin{array}{l}\text { Partnerships/Collaboration } \\
\text { Nature: Co-operation }\end{array}$ & $\begin{array}{l}\text { Various types of funding schemes: direct funding; } \\
\text { contracting-out; technical exchange on condition of } \\
\text { funding; } \\
\text { Nature: Competition }\end{array}$ \\
\hline (b) Objectives & $\begin{array}{l}\text { Poverty alleviation } \\
\text { Human development } \\
\text { Avoid duplication of efforts } \\
\text { Scale up of practice }\end{array}$ & $\begin{array}{l}\text { Increasing disbursement of funds to local NGO due to } \\
\text { aid policy and legitimacy of engaging with civil } \\
\text { society; } \\
\text { Financial efficiency; Market penetration } \\
\text { Maintain area of territory } \\
\text { Increase reputation }\end{array}$ \\
\hline $\begin{array}{l}\text { (c) Selection of } \\
\text { partners }\end{array}$ & Application procedure & $\begin{array}{l}\text { Consider bilateral aid policies about priorities in } \\
\text { relation to those of other funding schemes; discussion } \\
\text { with ministries. } \\
\text { Through personal network/ reputation/ previous links } \\
\text { Partners' capability to manage funding scheme as a } \\
\text { requirement. }\end{array}$ \\
\hline $\begin{array}{l}\text { (d) Practices of co- } \\
\text { ordination }\end{array}$ & $\begin{array}{l}\text { Technical co-operation } \\
\text { Consultancy }\end{array}$ & $\begin{array}{l}\text { Focusing on producing reports and meeting deadlines } \\
\text { Compromise approaches to meet the requirement of } \\
\text { many parties }\end{array}$ \\
\hline $\begin{array}{l}\text { (e) Advantage of } \\
\text { partnership }\end{array}$ & $\begin{array}{l}\text { Co-ordination of practices and resources } \\
\text { Mutual learning }\end{array}$ & $\begin{array}{l}\text { Conflicts due to the diversity of organisational culture, } \\
\text { capacity and approaches. } \\
\text { Struggles within the hierarchy of status and over } \\
\text { resources, } \\
\text { Competition in maintaining identity and autonomy, } \\
\text { Hesitation to change/accept new learning }\end{array}$ \\
\hline
\end{tabular}

As the case studies suggest, the patterns of relations between organisations are many and varied, despite the fact that they are often described in similar terms, using the words such as 'partnership' and 'co-operation' (as shown a in Table 5). The rhetoric of policy documents and project plans states that the purpose of IORs in aid sector partnerships is to reduce poverty, improve the well-being of people, in particular women, through collaboration with NGOs. At a field level, there is concern to avoid the duplication of effort and to scale up the activities (Table 5, b). These motivations cover up the various more practical intentions of the different actors. The intentions of donors working with local partners are often criticised. 
As Bassler and Wisses Smit remark:

Some partners, even with the best of intentions, may promise more than they can deliver. Some donors are more interested in being recognized for their own distinct contribution than in collaboration (1997: 37).

The selection of partners is influenced by bilateral aid policy objectives, and by the capacity of local partners to implement these, in terms of financial stability, and through individual channels (Table 5, c). For instance, in the case of JICA, the identification of its partners relies largely on previous personal linkages. During collaboration processes, interaction between funders and implementers often concentrated on producing reports with the required information and format. Collaboration between local NGOs appears to have to compromise on various aspects of organisational values, approaches, and mechanisms, which often results in conflict (Table 5, d). Diversity often created tensions between organisations. Sources of tension could also be found in diversities in organisational cultures, including at the interface with national cultures and values, international or local, and governmental or non-governmental status, specialisation and the length of establishment in the area. This tension seems to create competition about identity and autonomy in IORs, which appears to be the opposite of the expectation of partnerships (Table 5, e).

\section{Conclusion: Comparative validity of observations}

This chapter has presented factors which emerged from stories of experience of IORs in two case study projects. The issues selected refer to aspects of organisational management, mechanisms and values, and to the capacities of individuals. The examples introduced here cannot be generalised. They are 
limited accounts of stakeholder experiences associated with these particular projects. However, such experiences are coming to be seen as familiar aid funded project processes. For instance, Harriss et al. (2000) discuss how to manage IORs in the aid sector in the light of just such experiences, including those of international organisations and local NGOs, in the context of other lowincome states. Preston and McCaffery (2002) analyse the complexity of IORS processes in social development projects in different parts of the world and many of their illustrations mirror those described here. As more research is undertaken into these practises, so the quality of its comparative validity will strengthen. To move on into new terrain, I want now to analyse the implications for learning in association with evolving partnerships. 


\section{CHAPTER 6 INTER-ORGANISATIONAL RELATIONSHIPS AND LEARNING}

\section{Introduction}

This chapter investigates the relationship between partnerships and learning in the case study projects associated with the patterns of inter-organisational relationships (IORs). Selected incidents drawn from field data are used in order to gain multiple perspectives on the issues. The IORs in this study were either co-operative and/or competitive, depending on an organisations' position in relation to its partners. My observation suggests that learning emerges in different ways, depending on the quality of the IORS. It may be supposed that the relationship between partnerships and learning interrelate in different ways during the different phases of the projects in question. In this chapter, I shall conceptualise incidents and a range of interactions with reference to organisational management strategy and gender.

In the first part of this chapter, the processes and outcomes of learning between members of different organisations are examined, using two well-known theories. The first applies the principal-agent theory to the relations between DFID and GILLBT, as well as JICA and PPAG. The second examines an incident between GILLBT and NFED, as well as between GILLBT and Action Aid (Ghana) in terms of the two prisoners' dilemma. Although these theories are widely applied in management and social psychology in general, they have been 
less generally applied to the analysis of learning. Organisational dilemmas were observed in each of the investigations of the case studies. This leads me to consider whether a new collaboration strategy should be introduced or whether the asymmetric relationships, determined by the structure of relations with the funding authority, should be maintained. The two theories are respectively applied to hierarchical and vertical structures of the case study organisations. They seem to provide useful parameters within which the processes of learning may be analysed, along with the effects of learning between organisations and the implications of this for the quality of future relations between them. The organisational relationships selected for this analysis are those between funding agencies, managing organisations, NGOs, implementing organisations, and host communities of the projects.

Moving down the case study project hierarchies to the field analysis, learning and relations throughout the project are conceptualised at the level of community. Interactions between local implementing NGOs and community authorities are examined on the basis of the relationship between 'outsiders' and 'insiders' within the community, so as to analyse tension and suspicion between them. Individual accounts given by adult women who participated in the projects provide yet another perspective on learning and relations between partner organisations. In this case, women's learning as a component of human development, and their relations with the external environment of their daily lives, are examined. 


\section{Resolving dilemmas between organisations: two approaches}

The two management theories put forward in this chapter examine issues emerging from co-ordinated hierarchical relations and competitive horizontal relationships. In order to conceptualise the dilemma of the donor and recipient being situated in a hierarchical structure, the principal-agent theory will be used. The principal-agent theory permits an examination of problem resolution in coordinated relationships. The two parties may have different interests and preferences. One side has superior financial resources, while the other has an advantage in terms of information. Here, I analyse whether this relationship characterised from the outset as asymmetrical will change through a process of inter-organisational learning. The dilemma will occur when each party starts breaking down the barriers imposed by their initial position and works towards a new structure of collaboration.

The second example focuses on the horizontal inter-organisational linkages between two NGOs and between one of the NGOs and the government of the country where the service is being achieved. Given the competitive environment in which they operate, the dilemma between them will be explored by using the game theory of the two prisoners' dilemma. This theory has been developed to explain how individuals (and organisations) at similar levels in a hierarchy chose to co-operate or compete with each other.

Using these two theories to analyse different types of collaboration will enable incidents of inter-organisational learning to be compared in the light of their 
capacity to solve the dilemma between competition and co-operation. My intention is to illustrate how phenomena observed relating to a single activity can be explained from multiple theoretical perspectives. There is no implication that these conceptualisations can embrace the whole complexity of the issues with which I am concerned. Rather, their selection at different points of the project's organisational hierarchy is attributed to the ways in which, to me, they lend themselves to the analysis of IOL. As such, I argue that the two theories are useful as conceptual benchmarks. First, I shall position the principal-agent theory in the context of this research. This will be followed by applying it to my case studies. The same procedure will then be followed with reference.

\section{Learning to co-operate with principals and agents}

Although the principal-agent theory was developed in the area of business management, it can usefully be applied to public and non-profit environments (Garson 2001). It is defined as follows:

The principal-agent problems arise when one party, the principal, wishes to ensure that another, the agent, acts in accordance with the principal's interests in situations where the agent has information unavailable to the principal (Mckintosh, 1999: 42).

This framework will be applied to actors in co-ordinated relations in the aid sector. I shall aim to analyse certain characteristics of organisational relations that indicate diverse aspects of power. I shall then investigate how this analysis relates to inter-organisational learning in taking the case of relations between bilateral donors and NGOs in Ghana as observed during my fieldwork. 
The issue highlighted by the principal-agent theory is how a principal should manipulate an agent to act in the best interests of the principal. Where the agent has different interests from the principal, the agent might act solely according to his or her own preferences and interests, rather than according to the wishes of the principal. This occurs where the agent has an informational advantage over the principal, since the principal is unable to monitor the agent's action perfectly. For instance, in a relationship between an employee (agent) and an employer (principal), the employer may wish to induce the employee to invest greater effort in his/her job, but the employer cannot check what the employee is doing all the time. The relationship between the owner of a firm (principal) and the manager of the firm (agent) provides another example of a principal-agent problem. In this case, the owner wants the manager to follow certain policies to maximise profit. The manager may have different interests, which would maximise his/her own salary, rather than the profit for the firm. According to the theory, the dilemma is caused by informational asymmetry and by the diversity of interests between the principal and agent. In order to make the agent act in the principal's interest, the principal builds incentives into a contract, which binds both actors, and which designates what needs to be done for mutual interests to be served.

In the aid sector, the relationship between donors and recipients can be analysed using this framework. Killick (1997) claims that the relationship between donors and recipients has a hierarchical character, because the donor uses superior financial resources to induce the recipient to undertake actions. The relationship 
is also based on imperfect and asymmetrical information, as the recipient is always in a position to know more than the donor about its real performance and intentions. Although donors monitor and supervise programmes and projects, they rarely have complete knowledge of recipients' implementation procedures. Aspects such as "ignorance", "time-lags" and "noise in the system" make it impossible to deduce actions from results (Killick 1998:102). Since it is not feasible for the donor to monitor the recipient's every action, a problem of asymmetrical information occurs which can significantly influence the donorrecipient co-ordination.

The principal-agent theory provides a yardstick by which the donor-recipient coordination and source of the learning between them may be analysed. Given the different priorities of the principal and agent, the principal imposes objectives through a contract. The principal and agent theory has been used mainly in economic science. It can be criticised for not placing sufficient emphasis on cultural and psychological considerations (Garson 2001). In some writing, the preferences of the agent and of the principal are independent, and they do not change as the interaction between the two players proceeds. For the purpose of my argument I shall challenge the basic principal-agent theory by arguing that, in some cases, collaboration between the two players can modify their priorities. Later, I will support this statement with observations based on data collected during my fieldwork. The following questions will be addressed: is there any case where the distance between the interests of the principal and those of the agent can be reduced through the process of collaboration, so that the principals 
do not need to give incentives, as much as they may have to initially? If this happens, it might lead to a questioning of the implications of the original relationship. It might also lead to a greater focus on factors that can bring their interests closer and whether the distance in the hierarchical relationship can be narrowed through collaboration. It is also necessary to ask whether the agent would be willing to give up part of this informational advantage, as the two organisations become closer.

My assumption is that sharing interests and values associated with learning from each other can go some way to solving the principal-agent problem. For instance, if the donor in the aid system (principal) does not attempt to solve the problem of asymmetrical information by frequent visits to the project site or other means, the relationship will remain as it is. Alternatively, if neither party is willing to share their experiences, to reach mutually agreeable objectives through dialogue, their different priorities will keep them apart. On the basis of the principal-agent theory, Figure 9 suggests ways in which learning may have the potential to change the model.

Figure 9 Modified principal and agent theory: effects of learning

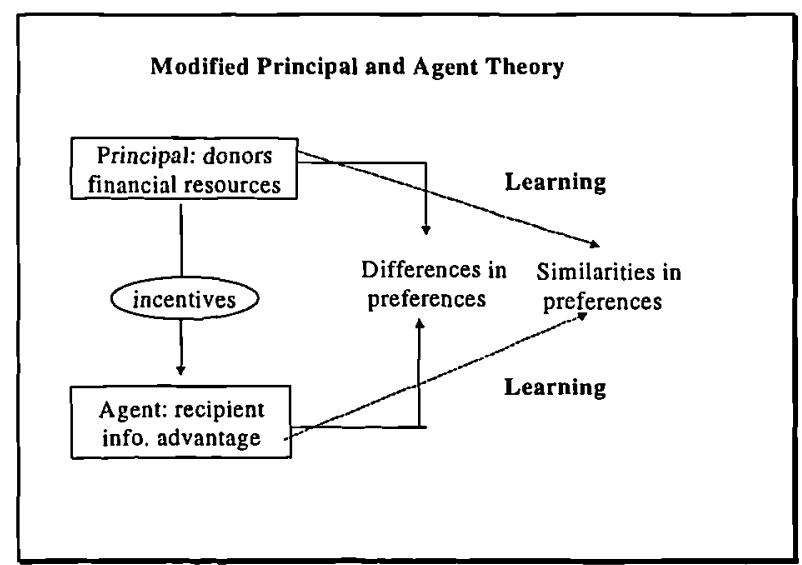


In presenting an illustration from the JICA funded project, I shall describe shifts in the relationship between JICA (principal) and PPAG (agent) over the period of the process of the project. In case of JICA and PPAG relationships, interorganisational learning between principal and agent occurred as a means of modifying the original structure as described in the principal-agent theory.

In contrast, the relationship between DFID (principal) and GILLBT (agent) did not change to the same extent.

Mutual learning to reduce hierarchy: the case of PPAG and JICA (Ghana)

One of the objectives of the Community Empowerment Programme (CEP) under which JICA funded the activity of PPAG is to encourage both partners to become more involved in the design and implementation of the projects. Since for JICA (Ghana), the scheme itself (contracting-out to a local NGO) and technical cooperation for a participatory community project are new concepts, it learned both techniques and values from PPAG, which has rich experience in this approach. In setting up the project, JICA (Ghana) staff stated that they had learned from PPAG relevant information regarding the current situation of NGOs in Ghana, as well as experiences and lessons from phase 1 (IP1) and general procedures of disbursements in contracting out (Transcript 26 and 27). What they struggled with most was how to find the best way to achieve a balance between the limited financial capacity of PPAG and the requirements within the CEP contracts. During discussions between JICA and PPAG staff, which took place sometimes 
as frequently as three times a week, both parties worked out their possible mutual contribution by consulting with their own governing bodies. In the case of JICA (Ghana), it took some effort to convince JICA (Tokyo) to exempt PPAG from obtaining Bank guarantees and to offer PPAG either payment in part and/or a total advance payment. PPAG also learned from JICA (Ghana), which acts as an intermediary between PPAG and JICA (Tokyo), how to interest JICA (Tokyo) in their proposals (PPAG/JICA, 1999/2000). Frequent interaction between JICA (Ghana) and PPAG created opportunities for sharing information and learning each other's interests and values. JICA recognised the depth of PPAG experience of project implementation, and was willing to trust their financial management capacities. As a result, JICA applied its bureaucratic requirements more flexibly than was usually the case.

\section{Maintaining the hierarchy: the case of GILLBT and DFID}

In contrast with the JICA example, the hierarchical relations and asymmetry of information between GILLBT and DFID were preserved throughout the project. The reason for this may be assumed to be a lack of monitoring by DFID. At the time of my research, no single person from DFID (Ghana or UK) had paid an official visit to the project site or to the headquarters of GILLBT in Tamale, in Northern Ghana ${ }^{39}$. This lack of face-to-face interaction between GLLBT and DFID may explain why DFID required answers to detailed questions on GILLBT activities and financial management, which they would otherwise have been able to observe for themselves. A GILLBT staff member commented that this attitude

\footnotetext{
${ }^{39}$ It has already been noted that there has been unofficial visits by DFID (Ghana and UK),
} 
created the impression that DFD did not trust GILLBT (Transcript 11). The judgement was also based on a comparison of GILLBT's experience with other donors, including NGOs in the UK. Their staff had engaged in much more frequent communication with GILLBT than was the case with DFID, and this had made for a much more flexible attitude to contracts.

Importantly, it has been difficult to find a structure with which to compare JICA (Ghana) and DFD (Ghana and UK) in respect of their relations with local counterparts. In the case of the JICA funded project, JICA (Ghana) acted as intermediate co-ordinator. Meanwhile, in the case of the DFID funded project, SIL (UK) had a similar organisational role to that of JICA (Ghana). Nevertheless, neither DFID (Ghana) nor DFID (UK) were involved in the GILLBT projects to the same extent as JICA was involved with PPAG. Hence, a direct comparison between JICA (Ghana) and DFID (Ghana and UK) on this issue would not be appropriate. However, a parallel examination of donors' attitudes using the framework of principal and agent theory suggests the possibility of making a connection between IOL and changes in asymmetric relationships.

There does seem to have been a shift on the part of GLLBT towards Income Generating Activities (IGAs), in response to clarification of DFID priorities. IGAs are intended to enhance financial well-being often through micro credit arrangements to support small-scale business activities with loans from an 
institution acting as a bank. GILLBT initially asked participants to pay back half of the total loan. This was because GILLBT's own practice was to treat half of the financial support being given as a loan, and half as a grant. This derived from its commitment to help the poor, rather than acting like a bank. DFID argued that the low level of IGAs outcomes and achievement was a key reason for refusing further funding. They claimed that the way GILLBT operated its IGAs did not help the poor in the longer term. It was this exchange that led GILLBT to collaborate with NGOs, specialising in IGAs, as explained earlier with reference to the SAT and GILLBT relationship. In this event, GILLBT came to realise that the full repayment of loans was feasible for the women participating in the scheme (Transcript 12). I infer that in this case, pressure from DFID gave GILLBT the opportunity to learn new skills, to strengthen their capacity and develop their programme. GILLBT seemed to view this advice positively. It has led to organisational change, with greater staff training and the establishment of new cooperation with another NGO. The feature of principalagent theory seems to be maintained in this case by DFID not monitoring as often as GILLBT would like, and DFID not changing its incentives. However in terms of inter-organisational learning, GILLBT did gain some lessons from DFID incentives and DFID priorities, which led to increased outcomes with regard to the particular projects being undertaken by GILLBT.

\section{Co-operating to learn}

Smillie and Hailey (2001) suggest that the most difficult alliance for local NGOs is with other NGOs rather than with donors or governments. The next 
illustration reveals the complexity of relationships between NGOs, which may create difficulties when they are working together. However, my case study shows that these difficulties of co-operation may also characterise the relationship between NGOs and government. The two prisoners' dilemma hypothesises that co-operating organisations will end up competing with each other as their best option, given their inability to co-ordinate their activities. Otherwise they will be in a situation where one side loses to the extent that the other gains. The theory is that neither party will benefit unless the actors are able to co-ordinate what they do in order to resolve the dilemma (see Table 6). Smillie and Hailey (2001), drawing on management literature, use the parable of the two prisoner's dilemma, in order to explain the reluctance of NGOs to cooperate with other counterparts.

Larsson et al's (1998) point with regard to the dilemma of inter-organisational learning is first that being a good partner invites exploitation by other partners attempting to maximise their individual appropriation of the joint learning, and second that such opportunistic learning strategies undercut the collective knowledge developed in the strategic alliance.

Larsson et al (1998) conclude that:

inter-organisational learning is likely to be hindered by a lack of either motivation or the ability to absorb and communicate knowledge between the partner organisations. The dynamics of power, opportunism, suspicion, and asymmetric learning strategies can constitute processual barriers to collective knowledge development. In contrast, the prior related interaction between partners, high learning stakes, trust, and long-term orientation are likely to empower the collective learning process (285). 
Table 6 The basic idea of the Two Prisoners' dilemma

\begin{tabular}{|l|l|l|l|l|l|}
\hline \multicolumn{2}{|c|}{} & \multicolumn{3}{|c|}{ Prisoner B } \\
\cline { 3 - 6 } & \multicolumn{3}{c|}{ Not confess } & \multicolumn{2}{c|}{ Confess } \\
\hline Prisoner A & Not confess & Left off lightly & Left off lightly & Punished severely & Reward \\
& & -2 & -2 & -10 & -1 \\
\cline { 2 - 6 } & Confess & Reward & Punished severely & Mercy & Mercy \\
& & -1 & -10 & -5 & -5 \\
\hline
\end{tabular}

Key:

e.g. -2 : prisoners will have a two-years sentence if this option is taken.

Table 6 shows the basic idea of the two prisoners' dilemma (Mas-Collell 1995). The numbers show the probable period of imprisonment for the different strategy options. The hypothesised situation is that two people are arrested for allegedly committing a crime and are questioned in separate rooms. Each one is told privately that if s/he is the only one to confess, s/he will be rewarded with a light sentence of one year, while the other will go to a jail for ten years. However if s/he is the only one not to confess, s/he will have ten years in jail. If both confess, they will each be shown mercy with five years of jail. If neither of them confesses, they will each have a sentence of only two years. They both face the dilemma of what to do in order to have the minimum sentence. The dilemma is that to confess is always the best strategy from an individual point of view, whatever the other decides to do. If neither confesses, they will have only two years in prison. However, if one of them confesses, the other will have 10 years of punishment. Therefore, it is safest for each to confess, whatever the other one does. Following the two prisoners' dilemma framework, Table 7 shows the relationship between NGO A and NGO B when they have to decide whether to co-operate or compete and how the parameters of any inter-organisational learning occurs. 
Table 7 The NGOs' dilemma and its IOL consequences

\begin{tabular}{|c|c|c|c|c|c|}
\hline \multirow{2}{*}{$\mathrm{NGO}-\mathrm{A}$} & \multirow{4}{*}{ Collaboration } & \multicolumn{4}{|c|}{ NGO-B } \\
\hline & & \multicolumn{2}{|l|}{ Collaboration } & \multicolumn{2}{|l|}{ Competition } \\
\hline NGO-A & & $\begin{array}{l}\mathrm{B} \rightarrow \mathrm{A}, \\
\mathrm{C} \rightarrow \mathrm{A}, \\
\text { Knowledge of NGOB } \\
\text { was transferred to NGO } \\
\text { A }\end{array}$ & $\begin{array}{l}\mathrm{A} \rightarrow \mathrm{B} \\
\mathrm{C} \rightarrow \mathrm{B} \\
\text { Knowledge of NGOA was } \\
\text { transferred to NGO B }\end{array}$ & $\begin{array}{l}\text { Does not learn } \\
\text { Exploited } \\
\text { No knowledge was } \\
\text { obtained }\end{array}$ & $\begin{array}{l}\mathrm{A} \rightarrow \mathrm{B} \\
\mathrm{C} \rightarrow \mathrm{B} \\
\text { Knowledge of NGOA } \\
\text { was transferred to NGOB } \\
\text { \& new knowledge, C was } \\
\text { gained }\end{array}$ \\
\hline & & \multicolumn{2}{|c|}{ Both NGO A and NGO B gain new knowledge (c) } & \multicolumn{2}{|c|}{ Advantage for $N G O B$} \\
\hline & Competition & $\begin{array}{l}\mathrm{B} \rightarrow \mathrm{A} \\
\mathrm{C} \rightarrow \mathrm{A} \\
\text { Knowledge of NGOB } \\
\text { was transferred to NGO } \\
\mathrm{A} \& \text { new knowledge, C } \\
\text { was obtained }\end{array}$ & $\begin{array}{l}\text { Does not learn } \\
\text { Exploited } \\
\text { No knowledge was } \\
\text { obtained }\end{array}$ & Does not learn & Does not learn \\
\hline & & \multicolumn{2}{|c|}{ Advantage for $N G O A$} & \multicolumn{2}{|c|}{ Neither NGO A nor NGO B learn any thing } \\
\hline
\end{tabular}

Keys:

$\mathrm{C} \quad$ indicates new knowledge in both NGO A and B. This knowledge is created by combining what both $A$ and $B$ already knew.

$\rightarrow \quad$ the arrow denotes the direction in which the information and knowledge flows.

N.B. Table 7 is a modification of Larsson et al. (1998).

NGO-A experiences optimum new learning (C) when it behaves competitively and NGO-B co-operates. On the other hand, NGO-A learns least when it is collaborative and NGO-B is competitive in this mode, NGO-B learns most. When both NGO-A and NGO-B are competitive, neither learns anything nor gains information.

It may be supposed that not learning anything and being exploited is a worse scenario than neither organisation learning anything at all. Therefore competition may be better than collaboration from the point of view of NGO-B if the first option is taken. Here NGO-A gains an advantage over NGO-B in this form of relation since NGO-A acquires new knowledge, whereas NGO-B does not. If learning by one party gives it an advantage over the parties not learning 
anything (a specific example of this will be given below), this competitive stance can bring about a better long-term outcome than being collaborative. I will illustrate this argument with reference to the case projects in Ghana.

The relationships between NGOs: experiences in collaboration with NFED

There were several points in the case study projects when NGO relationships with other NGO partners and with counterpart departments of the host government seemed to conform to the two prisoner's dilemma. Here, I draw on a case relationship between a local NGO, GILLBT, the national government and an international NGO, Action Aid (Ghana). The relationship with the government will be discussed first, given that this experience influences GILLBT relationship with Action Aid. GLLBT has developed relationships to varying degrees, with local community organisations in each indigenous language project area. Also it has worked co-operatively for a decade with the Non-Formal Education Division (NFED) of the Ministry of Education, 1987 to 1997 (Phase 1) ${ }^{40}$ when NFED delivered the national functional literacy programme (see Chapter 3). Currently Phase 2 of this programme is running from 2000 to 2004. The difficulty of language policy experience in Phase 1 left GILLBT hesitant about co-operating further with NFED, and led to the decision to join a partnership with Action Aid (Ghana). The meeting at which GILLBT staff discussed further collaboration with NFED highlighted several issues relating to

\footnotetext{
40 NFED has implemented a nation-wide functional literacy programme in 15 Ghanaian languages the Modified Freirean Approach, using primers with 28 functional themes based on life skills, occupational skills and civic awareness. (NFED/Action Aid 1998) Some staff at GILLBT disagree with the idea of restricting the number of officially recognised languages to 15 when there are estimated 60 languages as GILLBT is committed to mother tongue literacy development.
} 
GILLBT's attitude towards organisational change. Firstly, GILLBT was reluctant to change, given its past experience with NFED. GILLBT no longer trusted NFED, and feared of losing its identity through accommodating to NFED's language policy and risking exploitation by NFED in the process. Secondly there was conflict within GILLBT between those staff who had attended the meeting with NFED and who were directly informed about the NFED plans and those who did not attend the meeting and were not otherwise informed. Thirdly, again within GILLBT, Ghanaian and expatriate staff had different ideas about what should be done, and made a very unequal contribution to the debate (Takahashi, fieldnote Nov. 2000). The staff report of the meeting reads as follows:

...All these plans by NFED were seen to have been imposed on our staff contrary to the meaning of collaboration. The Literacy Department also has its priority areas, which conflict with the NFED agenda (Anane 2000).

Several members of staff, at different levels within the GILLBT hierarchy, described their experience with NFED in negative terms. One of the regional coordinators noted that his colleague had hardly seen any NFED people in the community after NFED had initially set up classes and distributed books. He also said that NFED people had used GLLBT attendance statistics in their report submitted to their donor, the World Bank. GILLBT had supplied on request because "they are government people, we cannot refuse it" (Transcript 16). The co-ordinator's comment highlighted the fact that the government had a certain degree of authority and was respected by local NGOs, at least in theory. However his experience left him with a lack of trust in NFED. The problem may 
have been increased by the fact that NFED had more resources to distribute than GILLBT in the literacy programme. Both GILLBT officers and communities highlighted the difficulties of collaborating with NFED. NFED provided financial resources and material incentives, such as bicycles, and sewing machines, to NFED facilitators who worked as volunteers in GILLBT literacy classes. Some GILLBT facilitators became less motivated and others were tempted to work for NFED because of this (Transcript 11,14 and16). The transfer of facilitator allegiance affected morale in the GILLBT programme, lured as the facilitators were to join a newcomer's activity and to leave the old one aside (Transcript 54).

Strategic differences in literacy programmes between GILLBT and NFED could also be seen. They include the number of language groups involved and the general approach to literacy teaching. While the GILLBT language project assisted twenty-two language groups in learning mother tongue literacy, NFED selected fifteen of them, which were more commonly used in wide geographic areas. In terms of approach, GILLBT focused on enabling people to read and write, whereas NFED stressed the modified Freriean Methodology of functional literacy, the method used by Action Aid ${ }^{41}$.

All of this created a sense of competition between GILLBT and NFED at the community level and the staff were unwilling to work together. The unequal

\footnotetext{
${ }^{41}$ NFED's functional literacy program uses a primer and a facilitators' manual. It starts with analysis and discussion of composite picture depicting a situation, a key word, syllabisation, formation of meaningful words and sentences, numeracy, writing and development activity (NFED 1999: 4)
} 
relationships, associated with resources and organisational status, between the government and NGO, affected the two players' perceptions of each other. In addition, GLLBT's approach to voluntarism, relying in small measure on the autonomy and independence of communities, was in conflict with the collaborative strategy adapted by the better resources of secular agencies. They include the government itself and international NGOs such as Action Aid ${ }^{42}$. Past experience did enable GILLBT to find new ways of collaborating with NFED, but GLLBT became very careful not to allow itself to be exploited or to lose its identity. Over time, this led some GILLBT staff to become more competitive.

On the other hand, NFED did recognise the poor quality of community level collaboration with other organisations in Phase 1. Several staff in NFED stated that stakeholders at regional levels were consulted over the design of the programme. A member of the NFED staff commented as follows.

...the approach used did not actually help NFED to realise the maximum co-operation at grassroots level, because the collaborations were done at the national level where you had all the top people, but they did not pass down to the community level of their workers so that they could be actually partners at community level. ... I would say that this would be one of the difficulties in the $1^{\text {st }}$ phase (Transcript 40).

At the end of Phase 1, the World Bank also pointed out that NFED was not satisfactorily including the local authority within their programme. For this reason, NFED entry into the community had failed (Transcript 41). The World Bank's mass literacy strategy seemed to be prominent in the planning report, and Ministry staff seemed encouraged by NFED's collaboration with other NGO

${ }^{42}$ This will be discussed further later in the chapter. 
stakeholders. However, problems persist. They include a lack of regional level understanding and strategy for implementation.

At the start of Phase 2, taking account of the lessons learned in Phase 1, NFED asked district and regional offices to give them lists of NGOs and other organisations working in communities. This was to make sure that they began collaborating with all of these agencies from the very beginning. The idea was that the partnership would start at the district level, building up to regional and national level after that. NFED set up a district training team with all the other collaborating agencies, including GILLBT, to discuss the local situation and analyse how the different organisations working in the same community might complement one another. This allowed organisations with different specialisations to share their expertise. For instance, in a literacy class, the presence of one of the specialist collaborators allowed an in-depth elaboration of the issues raised. This approach helped members of the collaborating organisations to get in touch with people in NFED classes if they wanted to use them to carry out certain activities such as environmental and sanitation work. In this way, the partnership facilitated service providers' access to local people (Transcript 40). Regional and district level co-operation aimed to reduce the duplication of activities through monthly and quarterly meetings. These keep each organisation informed of what was happening in the community and of local needs and difficulties on which they might focus their attention. This was stressed as a new procedure in Phase 2, as distinct from Phase 1. 
After feedback from colleagues, NFED learned the best way to collaborate with local organisations. Instead of taking its programme to the community, NFED tried to harness local organisations already working at the community level to identify ways in which they might work to support each other to maximise their collective subjects. This behaviour seemed to be far from being competitive.

GILLBT's suggestions for Phase 2 collaboration with NFED included the need to have a clear description of roles and transparency in sharing resources, including finance, expertise and materials. The discussion paper written by GILLBT staff gives an impression of GILLBT protecting their activities and community resources, particularly in places where GILLBT classes already exist. The paper stresses that GILLBT should be allowed to continue existing activities, using its own facilitators and primers. It suggests that NFED ought to be responsible for training facilitators in techniques for developing critical participatory pedagogies as the preferred NFED approach. The paper also says that "NFED will not set up any competitive projects in the area" (GILLBT, 2000). As GILLBT had already developed teaching materials and primers, it was proposed that GILLBT share them with NFED, although there may be problem associated with the use of different orthographies. GILLBT required funding from NFED for printing, workshops, training and facilitators incentives. As long as GILLBT shared NFED primers and facilitators, as its technical contribution to the collaboration, it will require financial support from NFED. 
It was felt by NFED that there were some individuals, particularly expatriates, who were hindering GLLBT's collaboration with NFED. While the reasons for this are not clear, it may be supposed that fear of past experience and NFED's unsatisfactory consultation with GILLBT over the development of Phase 2 as criticised in the staff meeting (Takahashi, fieldnote Nov. 2000) had made GILLBT staff cautious. It was also noticed that national staff in GILLBT were enthusiastic about working with NFED, since this provided greater status, as well as increased personal benefits and interaction with government officers (Transcript 10 and 11). Relating to the two prisoners' dilemma, GILLBT and NFED had ended up feeling as if they were in competition with one another after Phase 1. Although they were supposed to be collaborating, there was conflict, especially at the ground level, between GILLBT and NFED staff over facilitators and informational resources such as statistics and photographs, which GILLBT claimed that NFED exploited unfairly. GILLBT came to distrust NFED, and NFED felt that GLLBT was being uncooperative (Transcript 41). From the start of the second phase, staff in each organisation had to resolve the dilemma between being competitive and co-operative.

The history of relations between NFED and GLLBT suggested a process of learning for more effective partnership in future. Building mutual trust was a priority to overcome past experiences. It would take several meetings between managers of each organisation for this to happen. The staff of GILLBT deliberated at length over whether GLLBT should put in the effort required by NFED and risk being exploited, or whether they should maintain their normal 
activities while making sure that they did not lose any benefit from their collaboration with NFED. For staff, it was crucial that GILLBT protect its identity when it was collaborating with the more powerful organisation. Issues, such as these relating to competition and co-operation came to a head when a decision had to be made over technical collaboration. This required agreement as to the integration of approaches and as to the distribution of responsibilities.

The next section will look in more detail at the technical collaboration between GILLBT and partner NGO, Action Aid (Ghana).

GILLBT's technical collaboration with Action Aid (Ghana) ${ }^{43}$

The collaboration between GILLBT and Action Aid began in GILLBT's Anufo Literacy Project, which has been implemented in Chereponi, further north of Yendi in the Northern region of Ghana (See Appendix 3 and 4 for map) ${ }^{44}$. At the beginning of the collaboration between GILLBT and Action Aid, Action Aid contributed financially to the activities, and GILLBT identified local needs and possible activities in GLLBT's Anufo literacy project. This arrangement arose as a result of GILLBT's much longer presence in the community than Action Aid and Action Aid's search for a social area in which to invest. Recently, Action Aid had wanted GLLBT to implement Regenerated Freirean Literacy through Empowering Community Techniques, (REFLECT). REFLECT is a structured participatory learning process which facilitate people's critical analysis of their environment so as to achieve sustainable and equitable development. In a 
REFLECT programme, there is no textbook or literacy primer, but a guide for literacy facilitators. Each literacy project cycle develops its own learning materials through constructing maps and diagrams that represent local issues and promote detailed participatory analysis (Archer et al., 1998). It implied a new form of cooperation, combining the best of their own and the REFLECT approaches.

Some difficulties arose in the process of doing this. They include the length of training of REFLECT facilitators and the lack of resources with which to pay facilitators while they were being trained. Then, the committee for writing manuals did not realise that the REFLECT training was longer than training in the GILLBT method. Therefore GILLBT staff learned the methods of REFLECT, as well as the different requirements for training REFLECT facilitators. Confusion occurred when GILLBT facilitators attended the training for REFLECT. Unlike GILLBT, Action Aid and NFED normally pay facilitators for participating in training and workshops. Thus GILLBT became the "bad guy"'(Transcript 10). Eventually through negotiation between GILLBT and Action Aid (Ghana), GILLBT did not allow Action Aid to pay GILLBT facilitators being trained, although the funding was available to do so. I assumed that GILLBT's decision was largely based on the lessons learned from the experience with NFED. Now GILLBT experiences with both Action Aid and NFED indicate the importance of harmonising the collaboration between powerful and powerless organisations, particularly in terms of finance, in order

\footnotetext{
${ }^{44}$ Anufo is the local language used in the area.
} 
to have a common set of objectives. In order to make the project sustainable, GILLBT maintained its strategy of not paying sitting fees for training. Action Aid (Ghana), meanwhile, stopped paying sitting fees in order to harmonise with GILLBT policy. Inter-organisational learning was seen to occur in this negotiation process between all actors. Action Aid (Ghana)'s capacity to collaborate with the local NGO did not solely mean providing a financial contribution, but also working within objectives and approaches agreed by all concerned.

When efforts were made to clarify procedures at the outset, there could still be confusion. One volunteer working for Action Aid's REFLECT, tried to supervise classes. This was not his responsibility, but that of GILLBT staff. This account suggests that a single individual has the capacity to create difficulty between collaborating organisations. The importance of individuals should not be undermined in understanding IOR processes.

Another problem mentioned by the member of GLLBT staff relating to the progress of the collaboration was that the person responsible at Action Aid (Ghana) was always away, and other members of the staff in the office were not able to take action or make decisions on his behalf (Transcript 10). She even felt that staff at Action Aid (Ghana) seemed not to trust each other, let alone people in other organisations. Such issues may have caused several difficulties between organisations and made the process of collaboration very slow. For example, when GILLBT wanted to run a training programme to last for more 
than one week, Action Aid (Ghana) would not confirm support to this immediately, although they agreed in principle. This was because GILLBT was not communicating their proposal to the person responsible in Action Aid (Ghana) and GILLBT did not have funds to take the initiative itself. Later this situation was resolved, but there were some periods when people were not paid for participating in this activity. This again indicates how individuals may determine whether collaboration is either competitive or co-operative.

In another instance, GILLBT appreciated the fact that Action Aid (Ghana) provided some assistance for training, not only for REFLECT but also for GILLBT activities. This collaboration was felt to be positive in that Action Aid (Ghana) was willing to let GILLBT pursue its own course of action, and use their original approach to literacy development (Transcript 10). Action Aid (Ghana) staff began to feel that the quality of GILLBT work for REFLECT might not be of a high enough standard. Most of the facilitators available in the community had only basic literacy (Transcript 10 and 23). Leading a discussion in REFLECT circles required a much higher level of qualification than that possessed by most GILLBT staff. To avoid tensions over this, Action Aid (Ghana) suggested not using GILLBT's own literacy classes, but to start as Anufo REFLECT circles in villages where literacy classes had not been previously held. REFLECT has its own purposes and enabling people to write and read is only one part of them. It was thought better to start working with totally fresh groups so as to ensure that participants benefited fully from the REFLECT approach. 
This led to the idea of combining the two methodologies as an experimental approach. The critical point here was that this strategy allowed Action Aid to experiment by using GILLBT as a resource to introduce REFLECT. This meant training GILLBT facilitators, who were already trained to teach how to read and write, in REFLECT procedure, instead of providing financial support, Action Aid (Ghana) introduced its approach to the community by using GILLBT's facilitators as its human resources. This does not imply equal collaboration. However, it became the most effective strategy to develop REFLECT at the community level. The way the staff of Action Aid (Ghana) described their collaboration confirms the bias; "we use the Anufo Literacy Project (of GILLBT) to implement most of our own programme"(Transcript 23).

As for the formal contract between Action Aid (Ghana) and GILLBT, although there was already collaboration, it was necessary to draw up a memorandum of understanding. In doing this, GILLBT emphasised the importance of holding regular meetings, based on their past experience of not being able to contact the person in charge at Action Aid (Ghana). GILLBT also had to show its financial statements and arrange for allocation to the Anufo Literacy Project to be accounted separately. Clarifying the financial arrangements between the two organisations was crucial for continuing collaboration. GLLBT staff had also noted that when GILLBT needed to talk and complain about something, as in the case of the man from Action Aid (Ghana) trying to take over a GILLBT responsibility, the person in charge had been available to solve the problem. The 
situation whereby there was no place to express a complaint or to consult about the collaboration had created a gap between the organisations.

According to the original theory of the two prisoner's dilemma, the game is a one-off activity. I argue that if the game were to be repeated, each actor would over time learn the best way to act in order to achieve optimal co-operation and harmonise co-existence. In the case of GILLBT working with Action Aid (Ghana), individual staff did learn from past experiences and shared this with other staff in other projects. Through such interaction, each actor in collaborating relations makes an effort to contribute and learn what to expect from the others. Table 8 shows some elements of the dilemma faced by GILLBT and Action Aid (Ghana).

Table 8 Examples of IORs and IOL with GILLBT and Action Aid (Ghana)

\begin{tabular}{|c|c|c|c|c|c|}
\hline \multirow{2}{*}{ GILLBT } & \multirow{4}{*}{ Collaboration } & \multicolumn{4}{|c|}{ Action Aid (Ghana) } \\
\hline & & \multicolumn{2}{|c|}{ Collaboration } & \multicolumn{2}{|c|}{ Competition } \\
\hline \multirow[t]{4}{*}{ GILLBT } & & $\begin{array}{l}\text { Leam REFLECT } \\
\text { Obtain Financial } \\
\text { support }\end{array}$ & $\begin{array}{l}\text { Access to local } \\
\text { knowledge and } \\
\text { resources }\end{array}$ & $\begin{array}{l}\text { Exploited } \\
\text { Fall behind in } \\
\text { identity race }\end{array}$ & $\begin{array}{l}\begin{array}{l}\text { Access to local } \\
\text { knowledge } \\
\text { resource }\end{array} \\
\text { Identity kept }\end{array}$ \\
\hline & & \multicolumn{2}{|c|}{$\begin{array}{l}\text { GILLBT and Action Aid (Ghana) gain } \\
\text { the best profit }\end{array}$} & \multicolumn{2}{|c|}{$\begin{array}{l}\text { Action Aid (Ghana) has advantage over } \\
\text { GILLBT }\end{array}$} \\
\hline & Competition & $\begin{array}{l}\text { Learn REFLECT } \\
\text { Financial support } \\
\text { Identity kept } \\
\end{array}$ & $\begin{array}{l}\text { Exploited } \\
\text { Fall behind in } \\
\text { identity race }\end{array}$ & Do not learn & Do not learn \\
\hline & & \multicolumn{2}{|c|}{$\begin{array}{l}\text { GILLBT has advantage over Action Aid } \\
\text { (Ghana) }\end{array}$} & \multicolumn{2}{|c|}{$\begin{array}{l}\text { Neither GILLBT nor Action Aid (Ghana) } \\
\text { gain any profit }\end{array}$} \\
\hline
\end{tabular}

GILLBT will gain knowledge of REFLECT and financial support from Action Aid (Ghana) if GILLBT decides to be co-operative. However this may risk a loss of identity, if GILLBT is able to use its own approaches and strategies. This includes teaching techniques and the policy of voluntarism. On the other hand, if 
GILLBT decides to be competitive with Action Aid (Ghana), they might be able to pursue their own teaching methods and policy, if Action Aid (Ghana) provides solely financial contribution by being co-operative. In this case, Action Aid (Ghana) will be exploited for its financial resources by GILLBT. In reality, the collaboration between GLLBT and Action Aid (Ghana) aims at best practice by combining the methods of GILLBT and REFLECT. When both parties contribute their input, with GLLBT's methods, local knowledge and human resources and Action Aid (Ghana)'s financial support and knowledge of REFLECT, each will learn from the other and will be better able to achieve the objectives of collaboration. Of course, if the two organisations become competitive, which has not in fact happened, neither will learn anything from the other. As far as my observation and interview data permit, each organisation seems to be seeking a neutral line, between competition and co-operation, through a careful consideration of the distribution of responsibilities. The fact that Action Aid (Ghana) allowed GILLBT to continue what GILLBT was doing showed Action Aid's respect for the integrity of GILLBT's approaches.

Examination of the relationship between GILLBT and NFED, and GLLBT and Action Aid has revealed some of the critical incidents which created dilemmas between them. The decision as to whether to be co-operative or competitive depended on several factors from the perspectives of GILLBT, including taking advantage of resources, a fear of being exploited, a consideration of what was best for local beneficiaries and maintaining organisational identity. The dilemma was also largely influenced by past experience as well as individual attitudes. 
The dilemma for both sides may have been caused by a doubt as to what the organisation gains from the effort, or whether the other may gain more. Learning is maximised when both decide to be co-operative, and where both contribute to their collaboration.

\section{Rigidity or flexibility for changes}

As pointed out in earlier chapters, IORs are affected significantly by degrees of rigidity in their regulation, organisational management cultures and individual capacities. As regards the relationship between JICA and PPAG, the regulations stated in the contract were modified by the effort of JICA (Ghana) staff to convince the JICA (Tokyo) office to let PPAG work within the limits of its financial and organisational capacity. The effort and motivation to learn on the part of JICA Ghana's staff as individuals played a crucial role in reducing the gap in power relationships between these bodies, as well as narrowing the gap between the actors' preferences and interests. In this case, inter-organisational learning occurred as a direct result of learning more about each other. Such learning was encouraged by the frequent interaction between different groups of actors. The enthusiasm with which some JICA staff called for a continued funding to PPAG after the project's completion was an indication of the level of trust between them ${ }^{45}$. This suggests that the parameters of the relationship lie beyond the basic features of the principal-agent theory. Past experiences between GILLBT and NFED made it difficult for them to seek to share financial, human and technical resources based on mutual trust, for a second time. The

${ }^{45}$ email message (April 01) 
learning relationship seemed to be influenced to a large extent by organisational status and resources as power indicators. The fact that NFED is a governmental organisation with large resources led both actors towards competition rather than co-operation. From the lessons learned, some members of staff gave the impression of struggling with the dilemma of whether to be competitive to protect their advantages and risk of exploitation or to commence a new relationship with optimistic view. As a modification of the two prisoners' dilemma, if the game were to be repeated, the actors would aim for co-operation without being exploited. This implies that co-operation will be achieved if partners use mutually acquired knowledge from the outset.

The technical co-operation between Action Aid (Ghana) and GILLBT has many elements that affect inter-organisational learning. Among them, individual roles should not be overlooked. Uncertainty as to whether to co-operate or not seem to be connected with personal as well as organisational conflict. To analyse this further, providing a clear statement here lies beyond the scope of the present research. However, it is clear that the complex array of issues related to past conflict between individuals, particularly between Ghanaians and expatriates, have to be understood at the heart of IOR and IOL processes. Some informants observed that such incidents may be the underlying cause of non-co-operating relationships. 


\section{Learning and relationships in the community}

In the final section of this chapter, learning and relationships between stakeholders in the case study projects are examined from the perspective of individuals at a community level. Several types and processes of learning are explored with reference to inter-personal and inter-organisational relationships. The section enquires into changes in practice favouring innovations introduced from outside the communities. In the process of introducing new approaches and methods, several incidents revealed that hierarchical social relations within communities play an important part in determining the ways in which individuals become involved in projects, and benefit from them. Observing gender relations, for example, shows how women confront the hierarchies of their environments in the community and the management structures of the projects in which they become involved. Networks have significant roles in complementing the constraints formulated through the ladders of hierarchical structure of organisations working for and/or in the community. These ladders of community structure were often so rigid that the project implementing organisations had to streamline each organisational actor's role when delivering the projects. As the projects developed, individuals in different positions and roles in the partner organisations within the hierarchies underwent a learning curve.

Gender relations: hierarchy and network at community level

Both the adult literacy classes of GILLBT and the primary health care programmes of PPAG sought to bring changes to women's lives within their 
communities. The guiding theoretical frameworks regarding gender analysis in development in recent decades have varied from Women in Development (WID) to Women and Development (WAD), then to Gender and Development (GAD) (Rathgeber 1990: Moser 1993). The oldest and most dominant perspective, WID, originated in the early 1970s, and focused on the need to integrate women into the economic systems through legal and administrative changes. In the mid 1970s, WAD focused on the relationships between women and development processes. Acknowledging that men's behaviour was influential in determining the position of women, GAD developed in the 1980s as a means of perceiving all aspects of women's lives (Rathgeber 1990).

Women in rural areas in Ghana, regardless of the region concerned, tend to be restricted in their life choices (Adu 1999). An adult woman within a rural community in Ghana is described as having multiple roles and responsibilities in the family as mother, grandmother, aunt, daughter, and wife (Korten et al. n.d). She is a trainer of children, both young and old, as well as an assistant at family activities, such as weddings, funerals and co-operation with co-wives. She is also a treasurer or money-keeper for her daughters, little sister and other younger female members of family. These characteristics of the rural woman in Ghana seem to be similar to those of women elsewhere. However, qualitatively these women in the community have much more work than others. The following account gained from a woman co-ordinator of the literacy project of GILLBT gives a snapshot of women meeting the project from outside.

First of all, to encourage women to attend literacy class and IGAs is very difficult. Some people hid as they thought that we were coming to disturb 
them. Because women did not know what was happening, they did not want to come out and they were already very tired at the end of day after all the work. She wakes up and sweeps the floor, bathes babies, cooks breakfast, goes to fetch wood for fire, cooks lunch, washes clothes, goes to work in farm, cooks supper...(Transcript 17).

Women work all day for their families. Yet at the same time they resist being at the bottom of the hierarchy at home and in the community. In the rural village, young women are discriminated against by elder women. Boys are more valued than girls, wives are obedient to husbands and elders are respected by younger males. Above all, rural communities in Ghana still practise chieftancy (Assimeng 1999), and village chiefs are located at the top of the hierarchy.

There are several incidents in each of the case study projects when community level events were changed so as to encourage the participation of women. Here first, gender relations between male and female are looked at in the light of their influence on project delivery. Then other actors in gender relations such as elders and chiefs are examined in the following section.

A female co-ordinator for GILLBT within the community recalled her experience as follows:

There are now one female and one male teacher in each village in order to increase women's participation in class. First of all, they did it in a mixed class, but it did not work. Women were not comfortable to sit with men in such a situation. So classes were made separately with the same sex of teacher (Transcript 17).

Such observations on gender relations at home and in the community had a notable effect on GLLBT approach in delivering literacy programmes. Several 
expatriates expressed the changes over the years in women's participation in literacy classes over the last decades.

When I was first observing literacy classes in the 1970s, few women were there. The situation like now which women and men are sitting in one class was almost impossible to imagine! (Transcript 65)

Not only were women regarded as being unequal to men, they were discouraged from participating in such activities by their own husbands. They did not like their wives going out in the evening and mixing with male students and male teachers. When they understood this cultural aspect, which discouraged women from coming to classes, GILLBT held classes for women and men separately. Based on this understanding of community level gender relationships, GILLBT also restructured its approach to income generating activities (IGAs). According to an informant who had been living in the community since the 1960 s, both males and females were initially involved in income generating activities. However, the activities did not work well for two reasons, as commented by a long-term committed expatriate for GILLBT. First, the Ghanaian members of GILLBT, who at that time were nearly all men, did not want women to have control over their own income in the bank. Secondly, the men in the community had a tendency to use the women's income, not for the benefit of their families, but for themselves. This seemed wrong since in direct contrast to women, men were regarded as lazy in their work (Transcript 7). Here too, GLLBT decided to create separate IGAs for women in order to protect their income.

PPAG staff had similar experiences of learning about how the process of implementing the project might be constrained by gender relations at community 
levels. A programme officer commented that IGAs seemed to start off on the wrong track and collapsed because community involvement was not very strong (Transcript 34). Initially the project required contributions from everyone. However no one was prepared to spend time on this extra work. A new strategy was sought to bring women together and to work in smaller units, so that they would profit directly and increase their contribution. Once again, this strategy demonstrated lack of gender awareness among project staff as the men withdrew women's profit, just as had happened in the GILLBT IGAs in Northern region. The PPAG response was to work through and not against the structures of community hierarchy. This meant approaching the men first before approaching the women, particularly starting with leaders of men and convincing them of the least for co-operating with what the women were doing so that the household would benefit collectively including the men (Transcript 35). In this way, through a response rooted in community culture, PPAG came up with the idea that IGAs for women had to start with an approach to men so that men would support women.

The next question is how participation in the project affected women's lives and how they saw themselves. GILLBT's commitment was to community well being, by increasing the level of literacy among women and men. Taking the case of an IGA group leader for women, its one of the leaders commented on the steepness of her learning experience. She said that through a gender-training seminar provided by GILLBT in its Women in Literacy and Development programme (WILD), she had learned that everything could be done by people of 
either gender, and that there should be no disparities in labour on the basis of gender. With this knowledge, she felt herself free to go and monitor the development of IGA activities in other villages and their impact on people's lives. She also had the skill to go and train people for a few days by using the knowledge of gender that she had obtained from her own training seminars (Transcript 17). The stories shared by such women with reflections on their own learning were inevitably woven together with their struggles with their own positions, in relation to those of men in the home and the community. The seminars and exchange visits arranged by GILLBT as the project implementing organisation led to the formation of women's learning networks, on the basis of sharing experience and skills. This was enhanced by successful outcomes of their own IGAs, and reinforced by the accomplishments of others.

In the case of PPAG, the following statement in the mid-term report refers to the obstacles that prevent women from benefiting from their projects, and how changes in traditional behaviour between men and women were achieved. The changes are observed by Community Based Distribution (CBD) Agents who volunteer to support activities of PPAG by disseminating information and contraceptives to community members.

The discussions also showed that, CBD Agents believe that because of their interventions, dialogue between spouses has greatly improved in their localities on various issues including sex, contraceptive use and the care of the family. It was explained for example that before the project, petty quarrels between spouses were quite common, especially due to the communication gap between spouses. Gradually spouses are developing that strong bond of mutual trust and understanding that is related to more open discussions on pertinent issues that affect them. Hitherto this was not quite common (PPAG 2000: 12). 
Hierarchy in communities: the elder and chief, blocking or driving force?

In direct contrast to the observation of hierarchy at community level as a constraint to women's learning, the following accounts introduced here suggest that the structures of community culture may also function as a driving force for community involvement in the IGA projects. This occurred in cases where individuals in positions of authority agreed to support the project. Chiefs and elders are respected for their decisions, and visiting a chief or an elder constitutes an important obligation for outsiders wishing to enter a community. I had several occasions to greet chiefs before beginning my community research, always accompanied by GILLBT or PPAG project co-ordinators (Takahashi, Field note 2000/1).

A PPAG report indicates how the staff of PPAG perceived community structures when introducing its IGA proposals (PPAG 2000). A member of PPAG staff interviewed the women who might become involved in IGAs about the activities they would like to undertake as a means of earning money, and if they would be interested in forming a club for this purpose. The women replied that the elders have to decide. The PPAG report states "They did not consider themselves to have the power to take decisions". The report mentions that the women showed interest in learning from facilitators in other communities. If they shared with them success stories from other areas, they could consider whether to form a women's club or not. This incident indicates how community hierarchy hinders women from taking actions to improve their own lives, but they might use evidence of positive experience elsewhere to change the attitudes of chief and 
elders. The report also suggests the need to intensify gender education for selfesteem, and the sharing of decisions between men and women. The following quotation from PPAG mid-term report (PPAG 2000) illustrates the changes of elders attitudes as they become aware of the importance of issues related to women's health.

What is unique is that the older women are advising their younger daughters to use contraceptives, which hitherto was difficult because discussions on sexual matters in the home among family members hardly existed. However, the untiring efforts of the project staff, they have overcome this difficulty of talking to their children particularly the youth on sexual matters. Moreover speaking out on sexual issues to their children against the time-tested norms has greatly assisted their adolescent children to take precautions against sexually transmitted diseases especially the life threatening HIV/AIDS. It was disclosed that the HIV scare was a major contributory factor that enable them to come out from their cultural shells (PPAG 2000: 20).

PPAG had found it essential to obtain the collaboration from chiefs and elders to deliver their project. Particularly for Maternity and Child Health (MCH) activities, staff needed the collaboration of chiefs and leaders to disseminate information about the MCH community services (PPAG 2000). It was confirmed in the PPAG report, that the majority of $\mathrm{MCH}$ beneficiaries had learned about the facility through communication with chiefs and elders, some of whom were extremely supportive of the project's activities. One chief I encountered was among the most enthusiastic. According to the people I met, he contributed not only land, but sand and wood for the construction of the clinic. He even worked on the building with other community members, as a communal labour team. At the time of my visit, an outreach clinic was being held in an open space in his palace ${ }^{46}$. The chief also attended the local meeting of the

\footnotetext{
${ }^{46} \mathrm{~A}$ mobile outreach clinic brings nurses to the villages and reaches marginal populations.
} 
PPAG steering committee, according to a notebook of a CBD Agent who recorded the agendas of meetings and attendance.

Other incidents in which chiefs played a role in promoting the project in the communities related to clarifying misperceptions and miscommunications between PPAG community workers and community members. One chief, for instance, was able to resolve the jealousy between volunteer CBD agents and community beneficiaries. There was an incident when community members withdrew support for the activities of $\mathrm{CBD}$ agents, as they thought the agents were being paid by PPAG (PPAG 2000: 17). As a leader of the community, the chief made a vital contribution clarifying the intentions of the activities of CBD agents and the nature of their work as volunteers. This prevented conflict in the community arising from introducing the project.

All of the above reveal a series of critical incidents in the efforts of women to become involved in the case study projects and how project staff, the women themselves and community leaders, might play critical roles in overcoming. The accounts refer back to previous organisational experiences of project processes and indicate a range of organisational and individual learning between the implementing agencies and the participating women. The implementing agencies learned to restructure their approach to community projects, so that women were encouraged to take part knowing that the community systems and values were learned to be respected. At the same time, this did involve men, so that they accepted the need to change traditional practice and ways of thinking. 
Women who participated in the projects had various opportunities to learn how to manage the constraints on their ability to benefit from the projects, as well as to learn new skills and knowledge for the well-being of themselves and their family. With the help of the implementing agencies in a supportive hierarchical structure, elders and chiefs became co-operative so that women might bring about change in their home and in the environment in the community. A deep knowledge of local reality, traditions and culture was crucial in enabling both PPAG and GILLBT to find a balance between respect for traditions and changing them. This was a crucial element leading to the projects' achievement of planned outcomes. The complex processes in implementing the projects were associated learning of those who involved.

\section{Managing community hierarchy}

The project implemented by PPAG was designed to strengthen community members' participation, particularly those who were key actors in community development in the area (see Appendix 5 for project structure). The District Assembly (DA) and District Health Management team were invited to occupy a central role in the management of the project. The project mechanism included meetings of a District Steering Committee comprising representations of all the government agencies within the communities, which were held to monitor overall project development. This led each agency to realise that they had a part to play in the way the project was being implemented (Transcript 36). A programme manager explained that by giving responsibilities to districts and 
including them in designing and planning the project right from the beginning, the project obtained a high degree of participation. He says:

... participation became more effective when people knew their roles..... that is how at least we work for agency (Transcript 36)

A significant change was seen in the relationship between PPAG and the DA in this particular project. For the first time in different PPAG projects, the DA contributed money towards the cost of the project implementation. Originally, government institutions like DA had perceived NGOs, including PPAG, as rich organisations, receiving limitless resources from international donors. Therefore the DAs did not consider giving financial support to NGOs (Transcript 34). However the commitment of the DA to the project at Birim North District changed this view when it became known how much had been contributed to the District by PPAG. PPAG was so surprised at the DA contribution that the news had spread quickly to other regions working with PPAG, which increased their interest in the project.

Through its commitment to the design and implementation of the project, the DA had significant responsibility for the project. Such a change in attitude may lead to improved ability to sustain the work of the project, which is often questionable what funding comes to an end. Although further funding was rejected by JICA, there seem to be a strong willingness to continue the project at community level, as well as in PPAG. The project has maintained its activities with the contributions from PPAG, and from individuals in the community. A further higher level of government commitment to the project is revealed in the fact that 
a Regional Steering Committee was included so that the project could be more centrally managed. When it comes to national policy issues, Districts have to discuss policy issues formulated at a regional level and previously decided in Accra, across various government departments. Therefore, the PPAG project added its own Regional Steering Committee, so as to make its activities compatible with the plans of Ghana's Eastern Region (Transcript 34). Efforts to invite heads of local government and involved organisations to join the discussion, were also made, as they were the ones to hold authority (Transcript 36). This was important in the development of the project, since it led to a wider participation of stakeholders at various levels, and an increased ability to work in partnerships within the political hierarchy at a community level.

It was observed that the more the local authorities were given responsibility and roles to act in the project, the greater the level of participation, as people became enthusiastic about the project and committed to it. This was found to be one of the crucial stages for the projects success. Since various stakeholders were engaged from the beginning of the project's cycle, they had an increased sense of ownership. This encouraged them to learn from other agencies in order to maintain a level of positive co-operation, which certainly led to the success of the project.

Learning between PPAG and the District Steering Committee occurred in the second phase of IP. Again the process of learning is associated with hierarchy between community and district authorities. PPAG learnt how to implement the 
project by achieving a high degree of commitments from local authorities. District Assembly representatives became more and more responsible for developments in their own communities, in this case associated with PPAG projects. This is evidenced by the cash contribution to the project by the District Assembly.

Getting to understand each other: new concepts in the same world?

Entering communities where there has been little interaction with the outside world creates problems for NGOs implementing projects. Inter-personal trust is not the only means to overcome them, but it appeared as an essential bridge between the NGO and community in the PPAG case study in Birim North District. CBD agents, normally members of the community where they worked, were trained by the NGO, but the community was sceptical about their activities. There were comments from CBD agents that people within communities did not take them seriously at the beginning. However, with careful explanation and gradual understanding, CBD agents succeeded in convincing them to trust their methods.

CBD agents had problems with some people who objected to modern contraceptive use. Even thought there was no organised opposition to contraceptive use, there were pockets of people who were unconvinced by their value. In many cases people used religion as an excuse. Two religious groups, Moslems and Christians (adherents to the Seventh Day Adventists), were reluctant to accept the idea. Over time, however, the intensive education and 
propaganda gradually began to influence their lives. Members of such groups, who initially would not take part in any such discussions, were shortly taking part, and some were beginning to use the appliances. This change was explained by the fact that, apart from the distribution of contraceptives, CBD agents also played a very important role in $\mathrm{MCH}$ activities. They not only referred cases to the $\mathrm{MCH}$ activities, but also provided education regarding antenatal care, delivery and post-natal services. During their home visits, CBD agents provided nutrition information, especially for lactating mothers and children. In this sense, CBD Agents had the opportunity to integrate culturally sensitive family planning issues into aspects of health, which were already fairly accepted by the people (PPAG 2000).

In this way, trust between CBD Agents and communities was gradually established through the range of actual activities they were developing, which involved a lot of interaction between the $\mathrm{CBD}$ and individual households. As the people in the community became accustomed to learning from the CBD Agents, they trusted the technologies they proposed. As such, CBD Agents were able to overcome the difficulties involved in changing people's values and beliefs. In this case, trust was developed through experience. In some cases, trust already existed between individuals and organisations, associated with social status and prejudice. For instance, people tended to trust religious leaders in the first place. However in cases like the one I am considering, if there is an institutional trust between organisations and individuals, it can be extended to further innovation 
and change. It was noted everywhere that trust was an important aspect of each relationship throughout the project.

\section{Learning horizontally}

One of the most significant learning experiences for the implementation of IP2 came from the knowledge and experiences of IP1. Learning between the two projects was part of the strategy for the development of IP2 practices. Approaches used in IP2 were developed from the knowledge of what worked and what went wrong in IP1. Besides some lessons practised in IP2, there were also new learning and changes made in IP2. IP2 succeeded in achieving a high level of participatory development, with a strong commitment on the part of government and NGOs at the field level. From the beginning of the design phase onwards, IP2 saw a shift to broader development aims, in addition to IP1's original focus on primary health care. Between IP1 and IP2, there had been several exchange visits on the part of staff of different levels, including programme managers, community based distribution agents and other volunteers. One of the leaders, who organised women's clinics and IGAs working in IP1, had been transferred to work in IP2 as a key person based on her experiences of IP1 (Transcript 34). This organised transfer of learning seems to have been effective in sharing knowledge and learning between the different bodies of the participating organisations.

A national conference, held in 1999, also provided opportunities both for IP1 and IP2, as well as other PPAG staff, to share their experiences and discuss issues. It 
gave an impetus at national and international level by presenting the achievements of each programme to representatives of government ministries and international organisations including bodies such as USAID and UNFPA (PPAG/JOICFP/JICA 1999). The learning processes of PPAG revealed the importance of adaptation to the local context, the ability to learn from errors, the applications of lessons from other geographical areas, and continuous efforts to build trusting relationships with the community.

\section{Conclusion}

In this chapter, different kinds of inter-organisational relationships have been conceptualised using several frameworks ranging from the perspectives of strategic management approaches and gender relations. Learning between organisations was shown as occupying different roles depending on how it is perceived in relation to overall inter-organisational interaction. In this chapter, incidents and theoretical models were selected as interpretative strategies, as discussed in Chapter 4 on methods. These incidents were used as tools to illustrate different conceptualisations of inter-organisational learning.

In the context of the cases using the framework of the Principal and Agent, IOL emerged as a trigger for change in agents involved in hierarchical IORs. Referring back to the discussion in Chapter 2, learning in these cases was situated in individual interactions across organisations, which stand as communities of practice (Hildreth and Chris 2000). In the case of horizontal IORs based on the model of two prisoners' dilemma, IOL was employed as a 
means of exploiting partners in competitive IORs (Larsson et al., 1998). In another, IOL is the process of judging the quality of partners whether or not it is possible to co-operate on the basis of trust. This process involved non-learning and non-reflective learning, which refused the potentials of learning if it is not applied to social reality (Jarvis, 1995).

In taking account of hierarchical gender relations at the level of the community itself, women's learning experiences have revealed the scope for empowerment and improved well-being. Learning through the different project activities was seen as enabling women to improve their opportunities and develop the potential to change their lives, often negotiating the constraints of their position in the hierarchy of their environment. Their learning journeys suggest a number of paths taken according to the characteristics of experiential learning (Jarvis 1995).

From all of this, learning can be seen to affect IORs in many different ways. At one level, IOL enhances organisations so that they can bring their interests, objectives and strategic practices closer together. At the same time, IOL may imply maintaining competitive relations and not sharing expertise and other knowledge resources. At an individual level, as was observed at the community level, what is learned from different organisations relies heavily on the personal interactions of individuals and these were typically informal, casual and unaffected by organisational commitments. However, in order to gain access to the stakeholders at the bottom of an organisational hierarchy, attention also needs to be paid to the hierarchies within communities. Respect and understanding of a 
project and relationships between the stakeholders can only be gained if the authority of the community itself collaborates with the project implementer. In such situations, individual enthusiasm for collaboration with partners determines the extent to which projects can attract participants. In the processes of building new relations between community and outsider, trust in personal relationships becomes a key issue.

In sum, I have used quite two different approaches in Chapters 5 and 6 to make observations about what my data imply for partnership relations and learning. In Chapter 5, I analysed detailed structures and processes of each case study partnership and issues raised between partners. In Chapter 6, I experimented with different ways of conceptualising learning incurred in selected incidents from the interview data.

As a novice researcher, my struggle was to understand the process of critique and to present my findings from a critical perspective. This for a Japanese was quite an alien experience. In time, scholarly writing in social science showed me how to position micro level data from each partnership project in relation to more general debates. For me, this turned out to be an important learning moment. I incorporated it from a management perspective, into my analysis of two critical incidents. This provided some explanation of random events, but not of the overall picture. I then decided to seek a more general explanation for the large amount of data I had collected about projects and the communities with which they worked. Since the study of women and development had been an important, 
if unspoken, motive for my doing a research degree, I decided to conclude Chapter 6 with an exploration of the gender parameters of partnerships and the learning within them at a community level

My aim was for these three conceptualisations to illustrate instances of learning experiences between the case study organisations across their hierarchical and vertical IORs. Time and word-space permitting, I could have used many other conceptualisations in this way, relating to any number of events. While this chapter conceptualised the connections between IORs and IOL using previously established frameworks, the following chapter analyses them from perspectives of how individuals shape the IOL processes. 


\section{CHAPTER 7 LEARNING IN PARTNERSHIPS}

\section{Introduction}

In this chapter, I provide a theoretical exposition of the linkage between interorganisational learning (IOL) and inter-organisational relationships (IORs) on the basis of the analysis conducted in the earlier chapters. In Chapters 4 and 5, attention was paid primarily to the implementation of the case study projects, with a detailed explanation of accounts of IORs. Chapter 6 provided a different conceptualisation of incidents involving IOL and IORs in selected situations. I shall refer back to the way in which individual learning, organisational learning and inter-organisational learning were conceptualised in Chapter 2. I shall, further, examine the issues raised in Chapters 4, 5, and 6, so that a deeper insight may be gained as to the connections between learning and relationships. This chapter explores the issues lying at the heart of the principal inquiry, namely the need to investigate the impact of IOL on the development of IORs. This inquiry is mirrored in many ways by a search for ways in which the nature of IORs may stimulate IOL. By focussing on these questions, the chapter will seek to ascertain the link between IOL and IOR from several perspectives.

Chapter 2 described the analytical dimensions of individual and organisational learning. Ideas were introduced to explain types and mechanisms of cycles of learning and how these developed between the level of individuals and 
organisations. With regard to the application of such types of learning, I identified modes of individual learning on the basis of their reactions to experiences. The image of cycles of learning occurring between individuals and organisations was drawn from the theoretical framework of Kim's mental models (1995) and from the communities of practice approach (developed by Lave and Wenger 1991).

My intention throughout this thesis has been to look at aspects of IORs in relation to IOL from the perspectives of individuals as well as organisations. To develop Chapter 7, I begin by selecting incidents of learning associated with identified types of inter-organisational relationships, to further explore the theorisation of the earlier chapters. The chapter focuses on the positions of different partner organisations within the hierarchy of organisational relations in the two case study projects and the learning between them. The processes of individual learning at a certain position within organisational co-ordination are then examined, given that the connection between the position of a task and its associated learning has been identified as a significant factor in leading the quality of partnership beyond its organisational boundaries.

Learning occurs in different situations and forms. It has been seen to emerge simultaneously and/or sequentially in a series of procedures embedded within organisational co-ordination. Learning, in most of the accounts I am concerned with in this study, was not planned. This type of learning can be categorised as situated learning (Lave and Wenger 1990). Discussion follows in order to see 
how types of individual learning bring to the level of organisational learning and changes. Ultimately, the implications as to how IOL affects the connotation of partnerships in IORs are sought.

The following section begins with learning as seen in the cases of hierarchical IORs followed by those that are horizontal. In Chapter 6, learning between donor and recipient was seen as being an element of the processes that occurred organically in project implementation. It was suggested in some cases that the efforts of individuals to initiate organisational change resulted from its learning inspired by pressures to change in the project delivery system and from the characteristics of the previous IORs. It was also suggested that organisational learning can alter the mode of IORs from hierarchical to less hierarchical relationships. The following examples, however, suggest other effects of individual learning that might affect IOR hierarchies.

\section{Learning from inter-organisational relations}

\section{Recipients' learning from donor requirements}

Despite the fact that donors imposed their own requirements on recipients during the process of funding, especially regarding report preparation, there were certain degrees of recognition on the part of recipients that such processes carried with them a potential for learning. For instance, a member of GILLBT staff made the 
following comment about donor demands for reports formats and the questions to be answered,

We have to think about what we actually did not think about before. We learn something about evaluation about our project (Transcript 11).

Collecting literacy statistics from villages is a very difficult procedure. For instance, a lack of transportation makes it impossible to visit villages every evening, since they are located far from one another. Yet the NGO was requested to find out how many classes, teachers and graduates there were and how many people might benefit from new literacy classes. It has been noted by an expatriate literacy consultant that this task of collecting information made the NGO much more sensitive and accurate in their statistics later on (Transcript 11). However members of GILLBT staff also felt that being required to prepare these quantitative and qualitative indicators drew their attention to the strengths and weaknesses of their activities and the services they were delivering.

A member of staff within SIL (UK) commented on GILLBT's learning opportunities in relation to DFID requirements (Transcript 4,5 and 7). GILLBT's feeling that the DFID requirements were a difficult burden was well understood by SIL (UK), particularly when it came to financial reports. However SIL (UK) also regarded these experiences as useful opportunities for GILLBT to learn how to be systematic and professional, particularly in the area of accountability (Transcript 4). 
A co-ordinator of the Women in Literacy and Development (WILD) programme also found some recommendations given by the Canadian government agency, CIDA, useful. They encouraged women to get involved in management so as to increase the proportion of women benefit it from the project. They suggested the need for a Ghanaian supervisor, rather than an expatriate (Transcript 13). She notes that DFID funding, insofar as it contributes to the WILD programme, increased female learners to 40 percent compared to 10 percent before the DFID funding. Moreover, the donors' requirements on the technical side were found to stimulate a learning process in community participants, in the case of the income-generating programme of GILLBT (Chapter 6).

In the funding relationship, there is often a dilemma in terms of whether to listen to what the donor requests or whether to pursue what the NGO aims to do. However, resolving such a dilemma can also be a learning exercise which enables the NGO to protect its identity. For instance, a complaint was raised by GILLBT that DFID changed its funding theme every year. This affected the way in which GILLBT prepared their applications for funds. Nevertheless, senior staff commented that this required change might offer an opportunity for GILLBT to rethink its own focus more clearly. GLLBT might also be able to reconsider whether it was worth their applying repeatedly to DFID and to change their focus to accommodate the strategies currently pronounced by DFID (Transcript 10). For instance, when DFID moved to support a rights-based approach, GLLBT faced the dilemma of whether they should also focus on this in their application for funding, even though GILLBT was already involved in 
rights-based work (Transcript 5). Conflicts were observed between expatriates and Ghanaians over this, attributable to a misunderstanding of the approach by certain expatriates who thought it implied an involvement in politics. The fear of this derived from past experience, when one of the expatriates had been jailed for a few days after being accused of inciting riots through the implementation of a literacy programme. A workshop delivered by SIL (UK) in Tamale helped GILLBT to understand the concept of a rights-based approach. This idea was not to set up opposition to the government, but to enable people to be aware of their individual rights within the country, as well as their responsibilities (Transcript 5).

Regarding the writing of reports to correspond to donor specifications, NGOs have to develop their capacity to meet required standards. In this process, they learn that if they do not satisfy requirements, they will lose future funding (Transcript 8 and 15). In the beginning, GILLBT did not apply for funds from bilateral governments. Their organisational history, based on SIL practices, had taught them that missionaries had to learn for themselves about the techniques of fund-raising, through trial and error experiences. More recently, given the resources it needs, GILLBT appointed someone who was capable of preparing bids and managing financial relations with bilateral government and nongovernmental donors (Transcript 8 and 15). As a result, GLLBT was able to build its organisational capacity, although the pressure to do so came from the incentive to satisfy donor requirements. 
As has previously been mentioned, the difficulty of preparing the financial reports for a series of donor organisations led each of the case study NGOs to develop new methods of simplification. An accountant of GILLBT and a director of PPAG commented on how they managed to meet multiple donor requirements. GILLBT started using the same report for all donors. In contrast, PPAG established a group of staff in its accounts department with particular responsibility for writing reports to meet the requirements of individual donors. Now PPAG is in the process of developing a system to establish common reporting procedures by using a new management information system. This will allow them to put all donor requirements together, and in the end, satisfy them all. Such a system is aimed to speed up report preparation and to ensure that the reports reach donors on time (Transcript 12 and 33). Establishing management systems in order to deal with the diverse requirements of multiple donors is a challenge for NGOs. However the process of developing a common system for financial management and reporting to all donors indicates a trend among the NGOs towards isomorphism. They are forced to adapt the system to donors' management cultures, which was a condition of obtaining funding in the first place.

In considering IORs between donors and recipients, there is a certain "dictatorship" through which donors impose their interests and modes of practice on recipients. This is achieved through the logical framework of the reporting system, which is a tool that stresses recipients' activities throughout the funding 
period (ODA 1995) ${ }^{47}$. Donor organisational structures also determine ways in which recipients interact with donors in the process of funding. For instance, local NGOs have little impact on the way in which donors disburse funds within their system, and even delay the process of implementation at ground level.

Some NGOs are given incentives by donors to increase the likelihood of funding. This seems to provide opportunities for learning new skills, even though NGOs often found this to be a painful procedure, as observed in the case of GILLBT. For NGOs, learning opportunities through paying attention to donors' requirements emerge coercively, although this may be seen as situated learning. However the different levels of NGO learning from such opportunities have been identified in the above accounts on the basis of the learning theories that were discussed in Chapter 2. In the case of GILLBT learning to write reports, a member of staff only corrected matters according to donor instructions. The process of correcting errors in this way is characteristic of single-loop learning based on Argyris and Schön (1978). On the other hand, the learning opportunity recognised when GILLBT had to rethink whether they wanted to change their focus in order to get funding is a characteristic of double-loop learning. In this situation, GILLBT staff had to face questions themselves about their own organisational values and the directions of their strategy, and then react according to their decision.

\footnotetext{
${ }^{47}$ The logical frame is a dynamic tool, for use throughout the project cycle. It is used to help clear thinking during project design, as a method of building understanding and commitment between all the people involved in the project and as a guide for monitoring project progress. It contains a hierarchy of objectives from development goals to immediate objectives, which lead intended outputs into inputs analysis. These objectives have to be clarified by verifiable indicators of achievement, value, means and assessment, assumption, risks and conditions (ODA 1995).
} 


\section{Donors' experiences of learning through commitment}

Accounts from the case studies show that participation in, and observation of NGO activities often provided individual members of donor organisations with learning opportunities, both at a personal and work-related level. Such individual learning has the potential to influence an organisation's mental models, as described in Chapter 2. In Kim's model (1995), the learning cycle, as explained by mental models, leads to further organisational change. According to my informants in the case studies, knowledge of various matters was obtained through interaction with members of partner organisations. It ranged from understanding social development in the context of Ghana, to techniques of implementing the project on the ground. The people working in the donor agency gained knowledge by attending NGO meetings, where they discussed problems of implementation and observing NGO activities within communities. This type of casual learning may be seen to depend on individuals' prior knowledge and experience, which help to develop the ideas from observations. As Jarvis (1995) stresses, individual biography has an effect on the reflection of experiences, as outlined in Chapter 2. Learning based on past experience also relied on individual intentions and enthusiasm about the activities involved when working with NGOs. This was the case with one member of JICA staff whose prior experience and knowledge in the health sector was mainly obtained from their position at a policy decision-making level in Ghana. After several visits to the field, he realised how poorly the policy strategy discussed at the level of ministries in Accra had been implemented at community level (Transcript 26). Another member of JICA staff whose prior profession as a secondary school 
teacher in Japan had certain common skills which could apply both to practices as teachers and as facilitators within communities (Transcript 27). She observed the highly developed skills that were required of facilitators. Their role was to motivate the people in the communities and help them to think that it was they who had started the project and who should be responsible for its continuation. This was not an easy task even for Ghanaian facilitators who shared the same cultural background as their people in the country, because they are regarded as community outsiders. The JICA officer commented that the facilitators' role was similar to that of schoolteachers. Both facilitators and teachers have to learn how to achieve their objectives from those whom they teach. She noted that she had learned these points by observing a facilitator, who was very good at motivating communities, without revealing his difficulties. She also learned about the difficulties that PPAG staff faced by being outsiders in the communities, when she attended the PPAG seminar. Here she had experienced reflective learning, as she committed herself to getting to know the activities and problems of colleagues in her partner organisation. Such learning may well motivate her to reduce difficulties that JICA caused PPAG relating to project implementation, particularly in respect of disbursement delays. Her reflective learning is a feature of experimental learning, which has the potential to develop innovation and change-oriented organisational learning (see Chapter 2 Argyris 1982). However, according to Kim's incomplete learning cycle (1993), such learning experiences relating to the implementation of projects on the ground are likely to be so fragmented that organisations might lose them when the individuals concerned leave the organisation. In the case of this informant, she was employed as being 
a local consultant for CEP. This means that she was not able to influence the development of JICA policy and strategy to anything like the same extent as JICA officers. Also there was no way for her to reveal her individual learning and experiences of difficulties through co-ordinating relations with partner organisations may not be revealed to others in JICA. Regardless of this, the fact that she continued to work in the same position within the newly funded project meant that her previous learning would be an advantage. How her accumulated knowledge would link to organisational learning, however, is not known.

Knowing and understanding partners' difficulties in implementing projects through observations of each other is very important for inter-organisational relationships. Accounts of these learning experiences indicate that individual sensitivity, understanding and opportunities to interact with the situation are crucial. This is reflected in the types of reflective experiential learning suggested in Chapter 2. The opportunities for this between donors and recipients are rare in cases where IORs rely on communications by non-personal interactions without face-to-face meetings.

\section{Learning from horizontal IORs}

The learning accounts within horizontal IORs have been described in the case of relationships between NGOs and between NGOs and government. By definition, technical co-operation is meant to stimulate the most active mutual learning. However, the case studies have shown that conflict linked to competition over identities and resources plays a significant role in preventing learning through 
IORs. Conflict and friction led to IORs being more uncooperative, than encouraging skills of learning from each other. As the practices in international joint ventures show (Pollard 2002, see Chapter 2), management style differences and strategic imperatives influence collaborative processes. The analysis of Chapter 6 also indicates how individual competitive relationships influence organisations and lead them to miss the learning opportunities

In the case of IORs between NGOs at community levels, the learning processes observed were found to be very complex, and in many cases, ineffective, as explained by using the metaphor of the two prisoners' dilemma in Chapter 6. This NGO experience is contrasted with the situation of private profit making organisations. Even learning between business-related organisations can be competitive if their principal aim is to increase each other's profit through technical exchange. In the non-profit aid sector, more competitive aspects come in to play. These include organisational identity, status, and domination, although their stated objective is to benefit the communities in which they are working. This is a portrait, which reveals underlying aspects of political and economic interests related to the aid system.

Disparities within organisations, affecting collaboration procedures, include their objectives, their technical approaches and their payment systems. With respect to inter-organisational learning, collaborative learning strategies with high transparency and based on prior relationships of trust (Larsson et al 1998), are significant factors in making IORs active for mutual learning, whilst recognising 
the essential diversity of organisational features. However, from my observations of bilaterally funded projects in Sub-Saharan Africa, it is more likely that organisations starting with diverse approaches have not yet found common strategies for co-operation, but rather, are still struggling with a number of differences.

In contrast, I observed several instances that stimulated the exchange of experiences and knowledge between organisations, especially among those who were involved in similar practices. In Chapter 5, it was emphasised that PPAG's organisational strategies and project structure intentionally increased learning opportunities between IP1 and IP2 activities. The national conferences on IP that PPAG held with invitations to a wide audience not only expanded PPAG's learning experiences to encompass national and international bodies, but also developed their wider social learning network. GILLBT also encouraged an exchange of experiences between a number of geographical language groups, particularly in the new activities of gender and development with organised workshops for literacy project staff (GLLBT 1998; 1999; 2000). Hence, the need to interact with other NGOs which are involved in gender issues dramatically increased (Transcript 17).

Casual and formal interaction between individuals and organisations involved in similar areas of project development becomes a significant driving force in interorganisational learning. Such learning through communities of practice mentioned in Chapter 2 as facilitation of learning should not be undermined as 
the source of IOL. The communities of practice to promote IOL can offer alternative solutions to competitive IORs.

\section{The Impact of individuals on the link between IORs and IOL}

Regardless of the different funding structures in the two case projects, there were organisations which played a role in co-ordinating relations between the funding and implementing organisations (see project map Figure 8 and 9 in Chapter 4). For the DFID funded project, SIL (UK) acted as a liaison organisation between DFID and GILLBT. Similarly, the JICA Ghana field office was situated between JICA head quarters and PPAG, in its hierarchically organised project structure. The two institutions enjoyed similar learning experiences, being located at the same levels of hierarchical IORs respectively in delivering the projects.

\section{The Case of SIL-UK:}

The role of SIL (UK) was to channel DFID funding to GLLBT and to monitor its expenditure. Through this funding mechanism, DFID funded a UK based NGO, which worked with partners in low-income states. In this position, SIL (UK) co-ordinated the two organisations: DFID and GILLBT, on any matters related to funding, since there was no direct interaction between DFID and GILLBT. The learning journey of SIL (UK) started by submitting a proposal to DFID. This had required learning about DFID funding systems, development priorities and monitoring and evaluation procedures (Transcript 4). SIL (UK) also learned how to communicate with GILLBT. A member of the SIL (UK) 
staff was also required to learn details of GILLBT activities, involving as it did multiple types of programme for literacy and development. For instance, by learning about GILLBT's IGA, SIL staff felt more capable of understanding the reasons why DFD did not approve of GILLBT's approaches, from a technical point of view. This experience enabled SIL (UK) to relate the GILLBT situation to DFID, and DFID' concern to GILLBT (Transcript 5). One of the most demanding tasks for SL (UK) as an intermediary actor was to reflect both organisations' concerns, as well as those of SL itself. This required learning about all organisations' priorities, constraints, issues and finally making sure that each organisation's needs were fulfilled. His role learning was not only about disseminating information, but also about interpreting thoughts and concerns between the two parties and themselves. A member of the SIL staff commented with regard to being in the middle of such a situation, " you either learn or die!" (Transcript 4) He observed that he learned to look for a shared agenda, instead of conflict, by reacting to differences.

Therefore the individual learning of members of SL (UK) led to the transfer of the meanings of the messages expressed by DFID to GILLBT in ways that GILLBT understood. In order to do this, members of SIL (UK) had to learn how to deliver messages appropriately, as a technical skill, and also to convey the right message required by the different organisational cultures. As has already been stressed in Chapter 2, learning and understanding the positions of organisations and their partners in a wider network should not be undermined. 
Such knowledge can, in effect, increase opportunities for learning from each other, thereby enhancing the quality of communications between organisations.

\section{The Case of JICA (Ghana)}

The learning experience of the JICA Ghana field office staff throughout the project also revealed the struggles of intermediate actors. As observed in the case of SIL (UK), the main difficulty was to find a balance between what was needed by local partners and what was required according to the regulations of the project. A member of JICA Ghana staff, for instance, expressed the fact that the more he understood the financial capacity of the local NGO and its ability to implement the project, the more he found that there were requirements and procedures of CEP which did not suit the situations of local partners. He pointed out the difficulty of compromise with local partners on how to implement a project, after having learned about both the local context and the requirements of the project funding from Tokyo. It was suggested that the newness of the JICA project scheme and the lack of staff experience in working with Ghanaian NGOs and their funding procedure led to a dependence of the donor upon local partners and any consultants available.

He also learned how the structure of PPAG was managed organisationally, in terms of its hierarchical decision-making process. He used this information to ensure that he did not intervene in its management structure when he contacted individuals in the organisations within the line of hierarchy. Paying respect to mechanisms of management structure of the partner was one of the important 
rules to consider in working collaboratively. His reflective learning was very rich. It allowed him to acquire knowledge on how to initiate IORs and how to implement the project under the newly funded scheme. There also seems to have been learning present in terms of how to approach a potential partner, how to use existing social networks in unfamiliar place with little previous experience, how to learn about local contexts in relation to proposed funding, and through his commitment, how to co-ordinate and negotiate with the needs of local partners and the requirements of headquarters. All this reflective learning could, in effect, contribute significantly to JICA's internal knowledge about how to fund and implement similar projects elsewhere. Again, the fact that this officer was on an external short-term contract might not have detracted from his learning experience. From an organisational viewpoint, his reflective learning was fragmented, according to Kim's ideal of the incomplete learning cycle (1993). Together with the learning outcome of his colleague in the office, also a locally employed consultant, their joint learning could be seen as opportunistic, given that organisational actions were taken on the basis of an individual's or a small group of individuals' actions, without challenging the basis of at the shared mental model of the organisations concerned.

Both the intermediary organisations SIL (UK) and JICA (Ghana) shared similar experiences of learning related to their positions in the projects' hierarchies. It was significant to note how much such learning processes impacted on the relations of donors and recipients. Indeed, multi-layers of personal interactions 
between organisations seem to have determined the directions of co-operation and trust-based IORs.

\section{Cultural difference}

Previously, the observation that expatriates in some cases seem to resist change was mentioned. However, another comment here raises the importance of opportunities given to Ghanaians to learn, because they might be better at adapting to international development work than expatriates. The following comments are given by an SIL expatriate working in GILLBT as funding officer and a member of SIL (UK).

Ghanaians should deserve opportunities to fail and learn. I learn that Ghanaians are able to understand and adapt what our sponsors needed even though they do not like it (Transcript 15).

Ghanaians are learning much quicker. Much more positive learning than expatriates. Ghanaians can accept them, but not expatriates(Transcript 5).

These comments on learning are not, of course, necessarily restricted to the Ghanaian nature per se. The perception of Ghanaian learning in this case is that because Ghanaians were new to the types of working introduced, they were more receptive than experienced expatriates. In partnerships, encouraging partners to learn carries with it unexpected risks, since failure may damage any potential positive benefits. However, an appreciation of partners' learning experience seems to be a vital step in challenging the constraints implied by asymmetric relationship. 
Having said this, there are certain recognised characteristics of Ghanaians in terms of aspects of learning culture and their relations to others. The Ghanaian scholar, Woode (1997), describes uniquely Ghanaian characteristics in the work place in his book Values Standards and Practices in Ghanaian Organisational Life. Woode (1997) points out that Ghanaians find it difficult to say either "Yes" or "No" (37). He argues that some people think the difficulty and inability to say "Yes" and "No" means that Ghanaian people are nice, even too nice to say "No" when it will hurt others' feelings. However, he further points out that this characteristic also implies their way of protecting themselves, and remaining inscrutable. If this is the case, the impression that expatriates have about Ghanaians being good at learning could be interpreted in an alternative way. If Ghanaians do not show their resistance or anger as much as do expatriates, they may be seen to be in favour of change. Twumasi (1986) observes that Ghanaians do not reveal their true wishes and fears, and that this causes a communication gap between themselves and foreigners. It is argued furthermore that in discussions, real intentions are not revealed, because some people may pretend to agree to an important issues whilst social relation they may shift their stand (cited in Woode 1997: 9). It is important to note how some particular characteristics and attitudes towards adaptation and behaviour in public have implications for cultures of learning, socialisation and the structure of inter personal relations established.

In the case of JICA, a JICA Ghana field officer commented on the lack of information exchanges and interactions with other donor countries (Transcript 
28). It was suggested that a lack of interaction was caused both by the organisation's mentality and limited individual enthusiasm to learn from others. A senior JICA officer further claimed that the Japanese were weak in each of these aspects. He went on to point out, referring to individual learning, that there are a large number of JICA staff who have language problems and limited ability, especially in English. A similar comment was made to explain the reasons why Japanese accounts of learning experiences are not circulated in the international community. The apparent lack of sharing such experiences is due to the fact that only a few Japanese resources are translated into English (Transcript 56). Interestingly, several JICA officers commented that Japanese ODA is very keen to know about what other donors are doing (Transcript 55 and 58). For instance, there is a large amount of research conducted by JICA with regard to the trends and activities of other bilateral aid agencies. In contrast, it would be very unlikely for DFID to approach JICA to learn what JICA is doing (Transcript 55). Although the enthusiasm of Japanese aid for learning was felt, several JICA officers recognised that the application of such learning was another story (Transcript 55 and 58).

Although learning between DFID and JICA at an agency level does not appear strong, learning between them at the project level on the ground was noted in the education sector in Ghana (Transcript 2). In the context of bilateral aid to Ghana, the issue of co-ordination in implementing a sector wide reform was often mentioned in respect of relation between Japan and the UK. There had been learning opportunities for both sides to know each other's position and the 
underlying reasons for this both at policy level in London ${ }^{48}$ and its practice level in Ghana (Transcript 2 and 58).

Comparing the learning culture between Ghana, Japan and UK is no straightforward task, since the diversities of learning from the perspectives of national culture was not the main purpose of this thesis. However, together with the discussion of attitude towards learning at DFID and JICA in Chapter 3, the above comments from informants do seem to imply certain features of learning based on individual national background ${ }^{49}$. The extent to which the characteristics of learning cultures influence the examination of the research questions has not been directly examined in this thesis, although disparities in the organisations in the light of their policy, strategy and organisational background have been mentioned in several places.

It is not feasible at this point to separate the uniqueness of organisations based on nationality and other factors of implementation, such as the structures of the different schemes. I argue that the case studies reveal that the differences in attitude towards learning cannot be justified by a single measure such as nationality.

\footnotetext{
${ }^{48}$ There was a meeting in London on UK-Japan Joint Learning Mission to exchange opinions about sector wide approach between UK and Japan at policy level, in order to understand better each others' stance and strategies as a process of aid co-ordination (Takahashi, M 2001)

${ }^{49}$ There is a range of literature investigating differences in national culture within organisations, such as Hofstede, G (1991)
} 


\section{Conclusion}

The study began with the question of whether learning how to co-operate in IORs can contribute to the achievement of partnership objectives. Indeed, learning about each other can build up a trust-based relation that might enable projects to be delivered smoothly, with more effective outcomes. This will happen at both individual and organisational levels. Inter-personal interactions can work to initiate trust relationships between organisations, since once relationships are set, individuals are motivated to exert pressure on organisations in ways that are commensurate with the wishes of partners. This often means that flexibility is required within an organisation's rigid structure. The degree of influence that such actions may bring to bear depends on the capacities of individuals and their positions in the organisations concerned. The IORs based on technical co-operation between local NGOs used learning as a means of achieving the objectives. However, conflict due to competition between organisations often changed the scope for benefit from potential learning opportunities. This may well happen, not only in collaboration with NGOs, but also in donor-recipient relations.

The study also finds that learning does not always work in improving IORs. It also works against achieving partnership. Learning not to co-operate or learning to resist changes, based on previous experiences and any personal reasons might indeed prevent IORs from developing. The exact outcome depends on who has the strongest voice in decision-making and organisational choice. When an IOR is vertical, the competition for resources and identities between organisations 
makes it difficult to learn to co-operate. When the IORs is hierarchical, there is little room for the upper actor to learn from the lower actor because the relationship is one of dominance and control. 


\section{CONCLUSION}

To conclude, I shall reflect on the reasons for undertaking this research, the approaches adopted to carry it out and issues arising from the methods I used to collect, analyse and present the findings from my data. I shall consider what the study implies for understanding partnerships and learning between their constituent organisations, in the aid sector and more generally. In the final paragraphs, I consider the directions that future research might take and factors which might be taken into account when investigating institutional arrangements of such complexity.

\section{Reflections on the issues}

It was my recognition that the structures of the aid system could create obstacles for those within it, as they struggle to provide assistance and alleviate poverty, that led me to undertake this research. I can now see that it may have been the multiplicity of such constraints, observed everywhere, over several decades, and voiced by service providers, community users and governments alike, that may have been crucial to the adoption of partnership as the vehicle for aid interventions at the end of the $20^{\text {th }}$ century. At the same time, as we have seen, partnership is on the surface a cosy term implying mutual support for mutual benefit. I set out to see how the idea of partnership translated in the practice of day-to-day relationships, within member organisations (at the level of their individual staff/members), and between them. 
The case studies reveal individual and organisational desire to protect autonomy, identity and resources within global aid co-operation as a whole. While the demands for more effective aid co-ordination have increased as a result of financial cutbacks in most donor countries, they continue to compete to benefit from their investment in international humanitarian and technical assistance

Decades of development experience embodied in the case study narratives lead me to the depth of the divide between the reality of partnership management and the unproblematised connotations of the word with its implications for equality of access to resources and the sharing of decisions oriented at the achievement of common goals. Instead, as we have seen, asymmetric power relations attributable to resources, status, nationality and networking constitute an endemic feature of today's aid partnerships. Rather than harmony, this is likely to result in friction between partners caused by differences in the mechanisms, structures and cultures of each organisation.

\section{Approaches to the study}

I placed myself in the study from the outset and have attempted to keep the reader aware of the importance of the topic to me as the research developed. I sought to conceptualise what this represented, as I struggled to engage with the debates of aid sector project processes and the ways in which they are seen to be influenced by the wider social and economic environment. 
My research journey began with little knowledge of theories of learning, organisations or how organisations might learn from one another generically or specifically, within the aid sector. In the end, however, the thesis has shed light on the complex structures of partnership and learning within them as a means of developing and enhancing the quality of relations in their member organisations and between them. I am not aware of other research that has as yet travelled this route. While contemporary aid sector studies have examined partnerships from the perspective of actors at different levels of the organisational hierarchy, only Preston and McCaffery (2002) have undertaken comparative case study analysis of the relationships between different categories of organisational actor. None has previously examined the extent to which learning has underpinned the development of such interaction in the first place or, in the form of reflective practice, taken them on to new levels of understanding. In this lies the originality of the present research.

With so many actors, individual and organisational, I could only plan an exploratory study, based on qualitative data. These were drawn from multiple resources including interviews, observations and analysis of unpublished and published documentation. From interview narratives $I$ have been able to conceptualise individual accounts of engagement with organisational partnerships and draw inferences from this about instances of situated learning. As an interpretative strategy, the analysis of critical incidents allowed me to explore issues and events highlighted by informants. This permitted a view of how individuals directly involved experience the quality of relations between 
partner organisations and the ways in which learning is a factor in their development.

Through inter-organisational learning, my study has used an innovative perspective to gain insights into the process of organisational change and adaptation for the purposes of better co-ordination between aid sector organisations. In focusing on processes of inter-organisational relationships in project implementation, the practice of partnership was also observed at an individual level. Since my research into partnership practices was from the perspective of a complex cluster of organisational relations, this led to an inescapable concern with the role of key actors, within the imaginary concept of partnership itself.

\section{Discussion of individual versus organisational dynamics}

Understanding processes of individual learning at work, through interaction with individual members of partner organisations is considered crucial to the understanding of day-to-day partnership practices. A number of elements are inter-woven in facilitating and inhibiting individual learning and, from there, in stimulating processes of organisational learning. From an individual viewpoint, past experience, motivation and capacity are key elements in activating learning. The ways in which these elements are subject to manipulation by organisational structures and wider environmental forces may enable individual learning to influence learning at organisational and inter-organisational levels. Influential factors may include the status of individuals within organisations, in terms of 
their occupation and position within an organisation's decision-making structure. It is inevitable that individuals will struggle to apply their knowledge to bring about organisational change, to overcome some of the very structural constraints which shape the conditions of their work.

To understand the engagement between partners, I have particularly examined the impact of individual learning on the nature of these inter-organisational relationships. While the limited capacity of individuals to influence change within an organisational structure was portrayed, individual informants made noteworthy contributions to the analysis of inter-organisational relations. As a result, I argue that such relationships are not a product of how organisations interact. Rather they show how individuals working in different partner organisations relate to each other. Such people explain their perceptions and impressions about their own organisation's partners, through the filter of their own personal experiences. Observations such as these differ significantly from the claims made by organisations, pertaining to the organisation's agency in partnership work.

A commonly held belief in organisations is that individual partners have a restricted capacity to change the schemes or systems of their own and other organisations. Now it seems that the more frequent the communication between organisations, the higher the mutual understanding, regarding partner organisations' environments and the constraints that they faced. Yet, understanding a partner's environment may play a significant role in establishing 
a trust-based relationship. This is likely to be the case when each organisation has full information about the other's lines of external accountability and how this might lead them to impose inflexible conditions on the relationship.

Understanding who controls the funding and who determines policy and strategy decisions is central to reducing the risk of error in casting blame for mistakes and poor collaborative support. At the field level, the view of the donor agency held by local implementers is largely shaped by how donor agency field staff interacts with local counterparts on day-to-day basis. Shared commitment and enthusiasm between the staff of different organisations stimulates mutual learning and this in turn leads to organisational learning. At the same time it is clear that the quality of inter-personal relations reflects the cultural structures of organisations and those of the management of funding schemes. For instance, donors with a field office in-country are likely to make significant efforts to communicate with their local counterparts, as compared to the ones who make infrequent visits.

Nor should the role of networks in facilitating learning through casual interaction that lies beyond the boundaries of organisations be underestimated. Social networks activate not only inter-organisational learning, they enable organisational relations to be established that are based on individual trust. Institutional frameworks are required to build formal organisational links that prepare the foundation for developing good relations and disseminating learning. At the same time, the capacity of individuals to accelerate and/or obstruct the development of opportunities cannot be ignored. 


\section{Contributions of my study to aid and development}

The research has sought to understand insider accounts of how partnerships work within the international aid sector. It has found that the different functions of individual learning have the capacity to stimulate and enhance the development of relations between partners, along both vertical and horizontal planes. Understanding how people learn from each other through such organisational interaction becomes essential to the analysis of how development works in the sector as a whole might be improved to reach its targets more effectively.

At the same time, such understanding reveals factors that inhibit and facilitate individuals, as they attempt to learn for themselves and as they seek to apply their knowledge to encourage learning at organisational levels. Without knowledge of this kind, the development of more effective poverty alleviation processes will be slow. Staff of development agencies, including multi-lateral financial institutions, bilateral agencies, local service providers and communitybased voluntary organisations, are aware of the little social impact their efforts may have, despite their organisations' long- term commitment and considerable financial investment.

For many it is the fundamental aid mechanism that explains this phenomenon. Since aid serves the financial interests of the international political economy it is structured to strengthen, rather than reduce the divisions between the poorer and richer world. 
The increasing integration of the world capitalist economy since 1945 has been supported by aid - that is, the transfer of public financial and technical resources from the industrialised countries to the peripheral countries. Aid is an integral part of international political and economic relations, and it reflects the rich/poor division within the global economy... For the countries of the South, aid represents their relative weakness within the international political economy, and provides a channel for external influence over their development policies and programmes (Youngman 2000: 96).

With such structured inequality, the question arises as to whether there can be coordination between actors designated as partners, if their primary financial interests lie elsewhere. There is also concern for the extent to which cooperation and increased human well-being in deprived communities is possible outcomes of the complex sets of relationships between what may be very unequal partners in an inequitable system. If the underlying strategy of the political economy of the aid-giving countries and the diversity of other stakeholder values, mechanisms and strategies become prime obstacles to aid co-ordination, what are the roles of individuals who plan such scenarios? Three questions arise as to what extent individual learning can rule this game.

This research provides a foundational enquiry and analytical tool that can be used in the investigation of more recent aid policy, emphasising as it does the importance of knowledge for development. Policy pressurising aid agencies to become managers of knowledge stresses the need to create mechanisms that enable information to be shared between all the organisational actors involved in development programmes. Although knowledge here frequently indicates that conveyed by information technology software, the processes of its dissemination reflect those of individual and organisational learning, within and between the 
many organisations involved. Knowledge is a product of learning. The policy of knowledge development cannot be ignored in investigations of organisational and inter-organisational learning in the context of inter-organisational relations on a global scale, such as those inherent to the aid sector. Given the disparities in inter-organisational relationships within the hierarchy of the aid system, learning and knowledge-sharing across organisational boundaries will mirror these issues, operationalising them in day-to-day practice. In this way, research such as this, which provides insights into the circumstances of individual and organisational learning, has direct implications for understanding the global knowledge being promoted in contemporary development policy.

All this has implications for poverty alleviation in poor communities of poor countries. First, there are restraints on the implementation of human development community projects associated with the structure of the aid system. Nevertheless, in such restricted circumstances, the resourcefulness of individuals to adapt organisational constraints to the needs of end-users may also be palpable. While my study placed emphasis on factors which reflect the process and quality of how a project is funded and implemented, it revealed the potential of individuals to contribute to change, at least within the layers of hierarchy in which they are situated. This does not imply that single individuals have the power to influence the overall culture of their organisation, let alone affect that of the wider system in which it is located. Secondly, organisational friction, which often occurs as a by-product of competitive relations, may serve to undermine fragile development initiatives. In such ways, this study enhances understanding 
of the underlying causes of poverty and the sometimes near chaos of delivering development aid. In this the combination of complex organisational structures and unpredictable individual behaviour constitute a significant part of the problem.

\section{Reflections arising from the study: suggestions and difficulties}

This thesis was not designed to investigate the way in which individual learning is systematised to contribute to organisational development. Nor does it provide direct evidence as to how individual learning from experiences within partnerships might bring about organisational change. Doing this would have required a longitudinal investigation study, possibly over several years. Even then, it may not have been possible to provide reliable evidence. Rather, the present study has provided a conceptualisation of individual learning, and has considered individual perceptions of processes of learning as they occur within and between partner organisations. For a more evidence-based approach, it might have been relevant to investigate systems of evaluation and knowledge management in the context of partnership projects. As far as this work is concerned, however, the approach used sought understanding of the meaning of individual conceptualisations of learning and partnership, as elusive concepts, difficult to define and measure.

It is opportune at this point to stress once again the difficulties of the studying process, when the structures of the research objects themselves are infinitely intricate. The present study has evolved as a means of comprehending the 
processes of international human development and the delivery of aid projects, which of themselves invite controversy from different viewpoints. The stated aims of development intervention and of those organisations involved in delivering aid are two-fold: to provide humanitarian assistance and to alleviate poverty. Hence, critiques of the substantive parameters of development schemes and the motivations of aid agencies have significant political resonance. In order to explore the processes by which partnerships come to be associated with individual learning, it is essential to consider the characteristics, of member organisations, their cultures, agendas and networks, and the likelihood that these will cause obstacles to collaboration and co-operation. Having examined and compared the complete organisational matrices of two bilaterally funded projects and the interaction between agencies and counterparts in three countries, the difficulties inherent in planning, executing, analysing and disseminating this kind of research project cannot be underestimated.

\footnotetext{
Afterwards

I want now to reflect on what I have learned, during my research journey, in relation to my points of departure described in the Introduction. If anything, the anger I experienced in the Philippines, at the struggle of poor people to survive and the futility of their remonstrating with aid system organisations has become stronger and stronger throughout the four years of my research. My personal interactions with individuals in the different organisations that represent the hierarchical matrices of the aid sector confirm my initial view of the limited
} 
capacity of individuals, however good their intentions to change the face of these realities. It seems to me that the entire situation crucially depends on how the aid sector is structured, within a globally unequal social system. 


\section{REFERENCES}

Adu, J. (1999) Women in human development, Accra: Horizon publications.

Aikman, S. (1999) Intercultural education and literacy: an ethnographic study of indigenous knowledge and learning in the Peruvian Amazon, Amsterdam: John Benjamins Publishing Company.

Anane, J. (2000) Literacy Department Regional Co-ordinators Meeting 24-26 ${ }^{\text {th }}$ Oct, 2000, Tamale, GILLBT.

Anderson, J. (1995) 'Learning and memory: an integrated approach', New York: John Wiley cited in D. Rollinson, A. Broadfield and J. Edwards (1998) Organisational behaviour and analysis: an integrated approach, Essex: Addison Wesley Longman.

Antonacopoulou, E. (1999) 'Developing learning mangers within learning organizations: the case of three major retail banks', in M. Easterby-Smith, J. Burgoyne and L. Araujo (eds) Organizational learning and the learning organization: developments in theory, London: Sage.

Araujo L. (1998) Knowing and learning as networking, Management learning, 29, 3: 317-336.

Archer, D., Cottingham, S. and Phnuyal, B. (1998) 'Reflections on REFLECT', in IED Participatory Learning and Action Notes: participation, literacy and empowerment, London: International Institute for Environment and Development.

Argyris, C. (1982) Reasoning, learning and action: individual and organizatinal, San Francisco: Jossey-Bass.

Argyris, C. and Schön, D. (1978) Organizational learning: A theory of action perspective, Massachusetts: Addison-Wesley.

Assimeng, M. (1999) Social structure of Ghana: a study in persistence and change, Tema: Ghana Publishing Corporation.

Aubrey, L. (1997) The politics of development cooperation: NGOs, gender and partnership in Kenya, London and New York: Routledge.

Bailey, C. (1996) A guide to field research, Thousand Oaks: Pine Forge Press. 
Barley, S. (1995) 'Images of imaging: notes on doing longitudinal fieldwork' in G. Huber and A.Van de Ven (eds.) Longitudinal field research methods: studying processes of organizational change, London: Sage.

Barnard, G. (2002)'Evaluating international cooperation: the new frontier: evaluation and organisational learning' [online] http://oneworld.org/thinkthank/evaluate/edit2.htm

Bassler, T. and Wisses Smit, M. (1997) Building Donor Partnerships, New York: the Open Society Institute.

Beaudy-Someynsky, M. and Cook, C. (1999) Japan's System of Official Development Assistance, Ottawa, Canada: International Development Research Centre.

Bebbington, A. and Riddell, R. (1997) 'Heavy hands, hidden hand, holding hands? Donors, intermediary NGOs and civil society organisations', in D, Hulme and $\mathrm{M}$, Edwards (eds) NGOs, states and donors: too close for comfort?, London: Save the Children/Macmillan.

Bell, C. and Newby, H. (1977) 'Introduction' in C. Bell and H. Newby, Doing sociological research, London, George Allen \& Unwin.

Bell, J. (1999) Doing your research project: a guide for first-time researchers in education and social science, Third edition, Buckingham: Open University Press.

Boeh-Ocansey, O. (1994) 'Ghana' in Education Research: education and training for the informal sector, Vol. 2, Case studies, London: ODA.

Bogdan, R. and Biklen, S. (1998) Qualitative research for education: an introduction to theory and methods, London: Allyn and Bacon.

Bradshaw, Y. and Wallace, M. (1991) 'Informing generality and explaining uniqueness: the place of case studies in comparative research', in C, Ragin. (ed) Issues and alternatives in comparative social research, Leiden: E.J.Brill.

Bresnen, M. and Marshall, N. (2000) 'Partnership in construction: a critical review of issues, problems and dilemmas', Construction Management and Economics, 18: 229-237.

Brett, T. (2000) 'Understanding organizations and institutions', in J, Harriss, T, Hewitt and D, Robinson (eds) (2000) Managing development: understanding inter-organizational relationships, London: Sage.

Britton, B. (1998) The learning NGO, Oxford: INTRAC (INTRAC Occasional papers series No.17). 
Broadfoot, P (1999) 'Not so much a context, more a way of life? Comparative education in the 1990s', in Alexander, $\mathrm{R}$ et al (eds.) Learning from comparing: new directions in comparative educational research, Vol 1. Oxford: Symposium Books.

Brown, D. L. and Ashman, D. (1996) 'Participation, social capital and intersectoral problem solving: African and Asian cases', World Development, 24(9): 1467-1479.

Brown, J. S. and Duguid, P. (1991) 'Organizational learning and communitiesof-practice: towards a unified view of working, learning and innovation', Organizational Science 2 (1), Special Issue: Organisational learning: papers in honour of (and by) James G. March (1991), 40-57.

Brundage, D.H. and Macheracher, D. (1980) Adult learning principles and their application to programme planning, The Ontario Institute for Studies in Education: Toronto.

Brunsson. K. (1997) 'Non-learning organizations', Scandinavian Journal of Management, 14 (4): 412-432.

Buchert, L. (2002) 'Towards new partnerships in sector-wide approaches: comparative experiences from Burkina Faso, Ghana and Mozambique', International Journal of Educational Development, 22: 69-84.

Burgess, R. (1990) 'Introduction', in R. Burgess (ed) Reflections on field experience, Studies in qualitative methodology 2 , London, JAI Press.

Burnell, P. (1998) 'Britain's new government, new white policy, new aid? Eliminating poverty: a challenge for the $21^{\text {st }}$ century', Third World Quarterly, 19 (4): 789-802.

Bytheway, A. J. and Dhillon, G. (1996) 'Significance of partnerships in the management of interorganisational systems', International Journal of Information Management, 16, 5: 369-380.

Cairns, J. C. (2000) Literacy and development assistance project, Ghana 19972000 Evaluation report, Tamale: GILLBT.

Carlsson, J. and Wohlgemuth, L. (2000) 'Learning in development co-operation as Introduction', in J, Carlsson and L, Wohlgemuth (eds) Learning in development co-operation, Stockholm: Almqvist and Wiksell International.

Carpenter, J (2000) Critical Incident Technique: method at a glance, [on line] http:medir.ohsu.edu/ carpentj/cit.html 
Castells, M. (1993) The rise of the network society, The information age: economy, society and culture, [The information age: economy, society and culture, Vol. I], Oxford: Blackwell Publishers.

Chambers, R. (1997) Whose reality counts?: putting the first last, London: Intermediate Technology Publications.

Chell, E. (1998) 'Critical Incident Technique', in G. Symmon and C. Cassell, Qualitative methods and analysis in organizational research: a practical guide, London: Sage.

Chisholm, R. F. (1998) Developing network organizations: learning from practice and theory, Massachusetts: Addison-Wesley.

Clark, J. (1995) 'The state, popular participation, and the voluntary sector', World Development, 23 (4): 593-601.

Coffey, A. and Atkinson, P. (1996) 'Concepts and coding' in A. Coffey and P. Atkinson, Making sense of qualitative data: complementary research strategies, London: Sage.

Commins, S (1997) 'World Vision International and donors: too close for comfort?', in D, Hulme and M, Edwards (eds) NGOs, states and donors: too close for comfort?, London: Save the Children/Macmillan.

Coopey, J. (1996) 'Crucial gaps in 'the learning Organization': power, politics and ideology' in K. Starkey (ed)(1996) How organizations learn, London: International Thomson Business Press.

Creswell, J. W. (1998) Qualitative inquiry and research design: choosing among five traditions, Thousand Oaks: Sage.

Cross, R., Parker, A., Prusak, L. and Borgatti, S. P. (2001) 'Knowing what we know: supporting knowledge creation and sharing in social networks', Organizational Dynamics, 30 (2): 110-120.

Crotty M. (1998) The foundations of Social Research: meaning and perspective in the research process, London: Sage.

Davies, C. (1996) The organisational culture of GILLBT: the effects of a multinational environment, MA course report (place unknown).

Davis, T. S. (1999) 'Reflecting on voluntary environmental partnerships: lessons for the next century', Corporate environmental strategy focus on environmental partnerships, winter 6 (1): 55-59.

Dawson, S. (1986) Analysing organisations, London: Macmillan Press. 
Denning, S. (2000) 'The springboard- the Introduction: storytelling for organisational change' [online] http://www.stevedenning.com/intro.htm

Denteh, J. (1997) Sustainability of literacy skills among neo-literates through post-literacy activities in selected districts of Ghana, Mphil Thesis, Institute of Adult Education, Lagon: University of Ghana.

Denton, J. (1998) Organisational learning and effectiveness, London and New York: Routledge.

DFID (1997) Eliminating world poverty: a challenge for the $21^{\text {st }}$ century, While paper on International Development, London: The Stationery Office Lid.

DFID (1998) Ghana Country strategy paper, London: DFID.

DFID (1999) The Civil society challenge fund: guidelines for applicants, Civil Society Department, London: DFID.

DFID (2001a Nov. accessed) 'About DFID' [online] http://dfid.gov.uk/AboutDFID/files/historical background.htm

DFID (2001b Nov. accessed) 'DFID working within Government' [on line] http//www.dfid.gov.uk/AboutDFID/files/working_within_government.htm

DFID (2001c Aug. accessed) Ghana: annual plan and performance review, West and North Africa Department, DFD (Draft: 26 February 2001).

DFID (2002 Aug. accessed) Country Assistance Plan: DFID Ghana, Developing our country assistance plan 2002-2005, DFID Consultation Document- July 2002. [online] http://62.189.42.51/DFIDstage/Pubs/files/cap_ghana.htm

DFID/Ministry of Education, Ghana (1998) Ghana Education Sector Support, London, DFD/Accra, Ghana Ministry of Education.

DiMaggio, P. and Powell, W (1991) 'The Iron cage revisited: institutional isomorphism' in $\mathrm{P}$, DiMaggio and $\mathrm{W}$, Powell (eds.) The new institutionalism in organizational analysis, Chicago: The University of Chicago Press.

Dunleavy, P. and O'Leary B. (1987) Theories of the State: the politics of liberal democracy, London: Macmillan.

Eade, D. (1997) Capacity-Building: an approach to people-centred development, Oxford: Oxfam Publication.

Easterby-Smith, M. and Araujo, L. (1999) 'Organizational learning: current debates and opportunities' in M. Easterby-Smith, J. Burgoyne and L. Araujo (eds.) Organizational learning and the learning organization: developments in theory. London, Sage: 1-21. 
Ebers, M. (1997) The formation of inter-organizational networks, New York: Oxford University Press.

Edwards, M. (1997) 'Organizational Learning in NGOs: What have we learned?' Public Administration and Development, 17: 235-250.

Edwards, M. and Hulme, D. (1992) 'Scaling-up in developmental impact of NGOs: concepts and experiences', in M. Edwards and D. Hulme (eds.) Making a difference: NGOs and development in a changing world, London: Earthscan/Save the Children.

FASID, Foundation for Advanced Studies on International Development (1998) Research on co-operation between $O D A$ and $N G O$,: Tokyo, FASID.

Finger, M. and Brand, S. (1999a) "The concept of the "learning organisation" applied to the transformation of the public sector: conceptual contributions for theory development' in M. Easterby-Smith, J. Burgoyne and L. Araujo (eds.) Organizational learning and the learning organization: developments in theory. London: Sage.

Finger, M. and Brand, S. (1999b) 'Learning in the face of power', Paper presented at Organizational Learning Conference, University of Lancaster, June 6-8.

Flanagan, J.C. (1954) 'The critical incident technique' Psychological Bulletin, 51: $327-58$

Forss, K., Cracknell, B.l, and Stromquist, N. (1998) Organisational learning in development co-operation: how knowledge is generated and used, Working paper, 3. Stockholm: Ministry for Foreign Affairs, Sweden.

Fowler, A. F. (1997) Striking a balance: a guide to enhancing the effectiveness of non-governmental organisations in international development, London: Earthscan.

Fowler, A. F. (1998) 'Authentic NGDO partnerships in the new policy agenda for international aid: dead end of light ahead?' Development and Change, 29: 137-159.

Fujisaki, T., Briscoe, F., Maxwell J., Kishi M. and Suzuki, T. (1997) 'Japan as top donor: the challenge of implementing software aid policy', Pacific Affairs, 1 (69) 4, Winter 1996-1997: 519-539.

Fukuda-Parr, S., Lopes, C., and Malik, K.(2002) 'Overview: institutional innovations for capacity development' in S. Fukuda-Parr, C. Lopes and K Malik (eds.) Capacity for development: new solutions to old problems, London: Earthscan/UNDP. 
Garilao, E. D. (1987) 'Indigenous NGOs as strategic institutions: Managing the Relationship with Government and Resource Agencies', World Development, 15, Supplement: 113-120.

Garson, D (2001 accessed) 'Principal-Agent theory: key concepts and terms' [online] http://www2.chass.ncsu.edu/garson/pa765/agent.html

GCCA, Ghana Common Country Assessment (1999) Draft, Accra: GCCA.

GILLBT (1995) Constitution and legislative manual, Tamale, GILLBT: pp25.

GILLBT (2000a) Fact sheet, Tamale, GLLBT: pp2.

GILLBT (2000b) Possible collaboration between GILLBT and NFED, Tamale, GILLBT: pp4.

GILLBT (n.d.) Working together with understanding, adapted from Kirby, J, Tamale: GILLBT, pp1.

GLLBT/SAT (n.d.) Memorandum of understanding cooperation agreement between Sinapi Aba Trust (SAT) and Ghana Institute of Linguistics, Literacy and Bible Translation (GILLBT), Draft, Tamale: GILLBT, pp 10.

Gnyawali, D. R. (1999) 'Inter-organizational learning dynamics: roles of networks on knowledge creation' Working paper, Blacksburg: Virginia Polytechnic Institute and State University.

Hagedoorn, J. (2001) Inter-firm partnerships - an overview of major trends and patterns since 1960, Strategic research partnerships: proceedings from an NSF workshop, National Science Foundation July 2001.

Harriss, J., Hewitt, T and Robinson, D. (2000) 'Introductions: why interorganizational relationships matter', in J, Harriss, T, Hewitt and D, Robinson (eds.) (2000) Managing development: understanding inter-organizational relationships, London: Sage.

Hatch, M. (1997) Organisation Theory: Modern, symbolic, and postmodern perspectives, Oxford: Oxford University Press.

Hellinger, D. (1987) 'NGOs and the large aid donors: changing the terms of engagement', World Development, 15, Supplement: 135-143.

Herbert, P and Robinson, C (1999) 'Another language, another literacy?: evidence from Africa', Written Language and Literacy, 2(2): 247-266. 
Hewitt, T (1992) 'Development countries: 1945-1990' in T, Allen and A, Thomas (eds.) Poverty and development in the 1990s, Oxford: Oxford University Press/The Open University.

Hildreth, P., Kimble, C. and Wright, P (2000) 'Communities of practice in the distributed international environment', Journal of Knowledge Management, 4 (1): 27-38.

Hjertholm, P., and White, H. (2000) 'Historical perspective: background and trends', in F. Tarp (ed.) Foreign aid and development: lessons learnt and directions for the future. London: Routledge.

Hofstede, G. (1991) Cultures and organisations: intercultural cooperation and its importance for survival, software of the mind, London: HarperCollins Business.

Honey, P. and Mumford, A (1992) 'The manual of learning styles, Maidenhead: Peter Honey, cited in D, Rollinson, A. Broadfield and D. J. Edwards (eds.) Organisational behaviour and analysis: an integrated approach, Essex, Addison Wesley Longman: 230-231.

Huber, G. P. and Van de Ven, A. H. (1995) 'Introduction', in G. Huber and A. Van de Ven (eds.) Longitudinal field research methods: studying processes of organizational change, London: Sage.

Hulme, D. and Edwards, M. (1997) 'NGOs, states and donors: an overview', in $\mathrm{D}$, Hulme and M, Edwards (eds.) NGOs, states and donors: too close for comfort?, London: Save the Children/Macmillan.

Hvalkof, S. and Aaby, P. (eds.) (1981) Is God an American?: an anthropological perspective on the missionary work of the Summer Institute of Linguistics, International Work Group for Indigenous Affairs (IWGIA)/Survival International: Copenhagen.

INTRAC (1999) 'The participatory approaches learning study (PALS) overview report for DFID', Oxford: INTRAC. pp73.

Inukai, I. (1993) 'Why aid and why not? Japan and Sub-Saharan Africa', in B, Koppel and R, Orr (eds.) Japan's foreign aid: power and policy in a new era, Oxford: Westview press.

IPPF (2001 accessed) 'Country File: Ghana' [online] http://ippfnet.ippf.org/pub/IPPF Regions/IPPF CountryProfile

IPPR, the Institute for Public Policy Research (2001) Building better partnerships: the final report of the commission on public private partnerships, London: IPPR. 
Isezaki K (1997) What is NGO-voice from the field Tokyo Fujiwara shoten (Original in Japanese)

伊勢崎賢治「NGOとは何か一現場からの声」藤原書店

Itou, M. (1998) 'Overview on Japanese NGOs' activities for international coopeartion'in Umahashi, Norio and Saitou, Chihiro (eds.) Handbook NGO, Ashahi Publisher: 234-247.

伊藤道雄「国際協力に携わる日本のNGO活動の展望」(ハンドブックN G O:市民の地球的規模の問題への取り組み。馬橋憲男、斎藤千宏編著) 明石書店

James, R. (1998) Demystifying organisation development: practical capacitybuilding experiences of African NGOs, Oxford: INTRAC.

JANIC (1996) Report of international symposium on Creating together a new Partnership: NGO support schemes contributing to people's self-reliance (910/Dec 1995) (Original in Japanese). 国際シンポジウム報告書 :

新たなパートナーシップの創造〜

「南」の人々の自立に寄与する政府のNGO支援策とは

JANIC (1998) Handbook for NGO, Tokyo: JANIC

Jarvis, P. (1995) Adult and continuing education: theory and practice, $2^{\text {nd }}$ edition, London: Routledge.

Jeffery, P. and Jeffery, R. (1997) 'Silver bullet or passing fancy?: Girls schooling and population policy', in C, Jackson and R, Person (eds.) Feminist visions of development: gender analysis and policy, London: Routledge.

JICA (1995) The country study for Japan's Official Development Assistance to the Republic of Ghana, Tokyo: JICA.

JICA (2000) 'Schemes: Community Empowerment Program' [online] http://www.jica.go.jp/english/activities/schemes/11com.html

JICA (2001) Community Empowerment Programme,

アジアー部計画課「開発福祉支援事業」( J |CA

におけるNGO支援事業), pp. 24.

JICA/PPAG (1998) Minutes of Meeting, March 1998, Accra: JICA, pp. 16.

Johnson, H. and Wilson, G. (2000) 'Biting the bullet: civil society, social learning and the transformation of local governance', World Development, 28, 11: 1891-1906.

Johnston, R. and Lawrence P. R. (1991) 'Beyond vertical integration - the rise of the value-adding partnership' in G. Thompson, J. Frances, R. Levačić and J, Mitchell (1991) Markets, hierarchies and networks: The coordination of social life, London: The Open University/Sage Publication.

JOICFP (2000) A guidebook for the development of sustainable CBD/CBS programs, Tokyo: JOICFP. 
Jones, P.W. (1988) International polices for Third World education: Unesco, literacy and development, London: Routledge.

Josselson, $\mathrm{R}$ (1995) 'Imagining the Real: empathy, narrative, and the dialogic self', in $\mathrm{R}$, Josselson and $\mathrm{A}$, Lieblich (eds.) Interpreting experience: the narrative study of lives, Vol. 3, London, Sage: 27-44.

Killick, T. (1998) Aid and the Political Economy of Policy Change, London: Routledge.

Kim, D. (1993) 'The link between individual and organisational learning', Sloan Management Review/Fall: 37-49.

King, K. (2000) 'Towards knowledge-based aid', Journal of International Cooperation in Education, 3 (2): 23-48.

King, K (2002) 'Banking on knowledge; the new knowledge projects of the World Bank', Compare, 32(3): 311-326.

King, K. and McGrath, S. (2000) 'Knowledge-based aid: a new way of working or new North-South divide?' [online], http://www.ed.ac.uk/ centas/futgov.home.html.

King, K. and McGrath, S. (2002) 'Knowledge-based aid: a for agency comparative study', Centre of African Studies, University of Edinburgh, Version 1. Draft for Discussion in SIDA, March 2002.

Kironde, J. and Yhdego, M. (1997) The governance of waste management in urban Tanzania: towards a community based approach, Resources, Conservation and Recycling, 21: 213-226.

Kolb, D (1984) 'Experimental Learning: experiences as the source of learning and development', Ebglewood cliffs: Prentice Hall, quoted in D. Rollinson, A. Broadfield, and D. J. Edwards, Organisational behaviour and analysis: an integrated approach, Essex: Addison Wesley Longman: 229-230.

Korten, D. (1980) 'Community organization and rural development: a learning process approach', Public Administration Review, September/October 1980: 480511.

Korten, $\mathrm{H}$ and members of the Dagbani Literacy Project (no date) 'A study on how, where and when to start with women's developments project' Tamale: GILLLBT.

Kvale, S. (1996) InterViews: an introduction to qualitative research interviewing, Thousand Oaks: Sage. 
Lancaster, C. (1999) 'Aid to Africa: So much to do so little done', Chicago: The University of Chicago Press.

Larsson, R., Bengtsson, L., Henriksson, K. and Sparks, J (1998) 'The Interorganizational Learning Dilemma: collective knowledge development in strategic alliances', Organization Science, 9 (3): 285-305.

Lave, J. and Wenger, E. (1991) Situated Learning: Legitimate Periperal Participation. Cambridge, UK: Cambridge University Press.

Levinson, N. and Asahi, M. (1995) 'Cross-national alliances and Interorganisational Learning', Organisational Dynamics Autumn: 50-63.

Levinthal, D. and March, J.G (1993) 'The myopia of learning', Strategic Management Journal, 14: 95-112.

Lewis, D. (n.d.) 'Bridging the gap? : the parallel universes of the non-profit and non-governmental organisation research traditions and the changing context of voluntary action', CVO International Working Paper 1, Centre for Voluntary Organisation, London School of Economics.

Lisle, E. (1985) 'Validation in the social sciences by international comparison', Cross-national research papers, 1 (1), 11-28, cited in L. Hantrais, and S. Manger, (eds.) (1996) Cross-national research methods in the social science, Pinter, London: 1-12.

Lieblich, A., Tuval-Mashiach, R and Zilbert,T. (1998) Narrative research: reading, analysis, interpretation., Thousand Oaks, C.A.: Sage.

Lister, S. (2000) 'Power in partnership? An analysis of an NGO's relationships with its partners', Journal of International Development, 12: 227-239.

Mackintosh, M. (1999) 'Contracts, information and firm's behaviour' in R. Simonetti, M. Mackintosh, N. Costello, G. Dawson, S. Himmelweit, A. Trigg and J. Wells. Understanding economic behaviour: firms, Milton Keynes: The Open University.

Makaram, S (1995) 'Interprofessional cooperataion', Medical Education, 29, Supplement 1: 65-69.

Mas-Colell, A. et al (1995) Microeconomic Theory, Oxford: Oxford University Press.

McGrath, S. (2001) 'Knowledge for development- the case of the department for international development', Paper presented at the Oxford International Conference on Education and Development, September 2001. 
McGrath, S. (2002) 'The British department for international development and knowledge-based aid', Compare, 32(3): 349-363.

McMichael, P. (2000) Development and social change: a global perspective, London: Sage.

McNeill, P (1990) Research methods, London: Routledge.

Ministry of Foreign Affairs, MOFA Japan (1995) We assist international volunteer projects! NGO support schemes in Japan, Tokyo: MOFA.

MOFA (1999a) Japan's ODA Annual Report Vol. I, II, Tokyo: MOFA.

MOFA (2000) Japan's ODA Annual Report, Tokyo: MOFA.

MOFA (n.d.) Global Issues Initiative (GII) on population and AIDS, pamphlet, (original in Japanese) 我が国の人口

ズに関する地球規模問題イニシアティブ

Moore, M. (2000) 'Competition within and between organizations', in J, Harriss, $\mathrm{T}$, Hewitt and D, Robinson (eds) (2000) Managing development: understanding inter-organizational relationships, London: Sage.

Morgan, G. (1990) Organizations in society, London: Macmillan.

Moser, C. (1993) Gender planning and development: theory, practice and training, London: Routhledge.

Musson, G. (1998) 'Life histories', in G, Symon and C, Cassell (eds) Qualitative methods and analysis in organizational research: a practical guide, London, Sage.

Nelson, N. and Wright, S. (1997) Power and Participatory Development: theory and practice, London: Intermediate Technology Publications.

Neuman, L. W. (1997) Social research methods: qualitative and quantitative approaches, 3rd edition, Boston: Allyn and Bacon

NFED/Action Aid (1998) International conference report, REFLECT on a large scale: challenges and prospects, $20^{\text {th }}-30^{\text {th }}$ July 1998 , Accra.

Nonaka, I. and Takeuchi, H. (1995) The knowledge-creating company: how Japanese companies create the dynamics of innovation, New York: Oxford University Press.

Non-formal education division (NFED) Ministry of Education, Republic of Ghana (1999) NFED at a glance, Accra: NFED. 
NRL, National Right to Life (Dec. 2002 accessed) 'Planned Parenthood: abortion provider' [online] http://www.nrlc.org/abortion/ppprov.html

O'Malley, D. and O'Donoghue, G. (no date): $N G O$ and the learning organisation, British Overseas NGOs for Development discussion paper, London: BOND.

Obirih-Opareh, N and Post, J (2002) 'Quality assessment of public and private modes of solid waste collection in Accra, Ghana', Habitat International 26: 95112.

OECD/DAC (1996) Shaping the $21^{\text {st }}$ century: the contribution of development co-operation, Paris: OECD.

OECD/DAC (2001a) Evaluation feedback for effective learning and accountability, Paris: OECD.

OECD/DAC (2001b) The DAC Journal development co-operation 2000 report, 2 (1), Paris: OECD.

OECD (Aug. 2002 accessed) About OECD, http://www.oecd.org

Orr, R. (1990) The emergence of Japan's foreign aid power, New York:

Columbia University Press.

Osborne, P. Stephen (1998) Voluntary Organizations and Innovation in Public Services, London: Routhledge.

Ouchi, W.G. and Wilkins, A.L. (1988) 'Organisational power', in A. Westoby (ed.) Culture and power in educational organisations, Milton Keynes: Open University Press.

Overseas Development Administration, ODA (1995) A guide to social analysis for projects in developing countries, London: HMSO.

Oxford English Dictionary (1989) Second edition, Vol.11. Clarendon press, Oxford: 278-279.

Pollard, D. (2001) 'Learning in international joint ventures' in M, Tayeb (eds) International business partnership: issues and concerns, New York: Palgrabe.

Population Council/PPAG (2000) An assessment of the community based distribution Programmes in Ghana, Accra: Population Council and PPAG.

Planned Parenthood Association of Ghana, PPAG (n.d.) PPAG in perspective (pamphlet), Accra: PPAG.

PPAG (1998) PPAG, [online] http://www.africaonline.com.gh/Ppag 
PPAG (1998) Progress report on Integrated family planning nutrition and parasite control project: phase II (Birim-North District, Eastern Region Ghana) April-August 1998, Accra: PPAG.

PPAG (1999) Annual report on Integrated family planning nutrition and parasite control project: phase II (Birim-North District, Eastern Region Ghana), April 1998-March 1999: PPAG Research Unit.

PPAG (2000) Mid-term evaluation report on Integrated family planning nutrition and parasite control project: phase II (Birim-North District, Eastern Region Ghana), Accra: PPAG Research Unit.

PPAG/JICA (1999/2000) Notes from correspondence message between PPAG and JICA (Ghana), Accra.

PPAG/JOICFP/JICA (1999) Report on the fourth national conference on the integrated family planning, nutrition and parasite control project (IP), 12-14 April 1999, Agona swedru (Central Region, Ghana): Catholic mission press (Cape Coast).

Presas, T. (2001) 'Interdependence and partnership: building blocks to sustainable development', Corporate Environmental Strategy, 8 (3): 203-208.

Preston, R. and McCaffery, J.(2002) Improving partnership: consultancy-related communication in complex projects: case studies of international educational development: International Centre for Education in Development, University of Warwick, Forthcoming.

Preston, R. and Preston, D. (2001) 'Researching environmental sustainability: knowledge, expertise and cross-national university partnerships in Europe and Latin America' (draft) paper presented in Society for Latin American Studies, Annual Conference, University of Birmingham, 6-8 April, 2001.

Raco, M. (2000) 'Assessing community participation in local economic development - lessons for the new urban policy', Political Geography, 19: 573599.

Ragin, C. (1987) 'The comparative method: moving beyond qualitative and quantitative strategies'. Berkeley: University of California Press.

Randel, J. and German T. (1999) 'United Kingdom' in I, Smillie and H, Helmich Stakeholders in Development: Issues in Government and NGO Relationships, Paris: OECD.

Rathgeber, E. (1990) 'WID, WAD, and GAD: trends in research and practice', Journal of Developing Areas, 24, July. 
Robinson, M. (1993) 'Governance, democracy and conditionality: NGOs and the New Policy Agenda' in Clayton, A (ed.), Governance, democracy and conditionality: what role for NGOs?, Oxford: INTRAC.

Rollinson, D., Broadfield, A. and Edwards, D. J. (1998) Organisational behaviour and analysis: an integrated approach, Essex: Addison Wesley Longman.

Ross, D. (1997) 'Aid co-ordination: donors, governments and NGOs', in Lynch, J. (ed.) Education and development: tradition and innovation, 4, Non-formal and non-governmental approaches, London: Cassell.

Sackmann, S. A. (ed.)(1997) Cultural complexity in organizations: inherent contrasts and contradictions, Thousand Oaks: Sage publications.

Sawamura, N. (2001) 'Local spirit, global knowledge: a Japanese approach to knowledge development in international co-operation', Paper presented at the Oxford International Conference on Education and Development, September 2001.

Schön, D. (1983) The reflective practitioner, New York: Basic Books.

Seddon, D. and Sato, M. (1997) 'Japanese aid and Africa', Review of African Political Economy, March 1997, 24 (71): 153-156.

Senge, P. (1990) The fifth discipline: the art and practice of the learning orgnization, New York: Doubleday.

Sifuna, D. (2000) 'Partnerships in educational assistance to African countries: rhetoric or reality?', Journal of International Cooperation in Education, 3(2): 321.

SIL (2002) What is SIL International? http://www.sil.org/sil/

SIL International (2000) Annual literacy report 2000, Nairobi: SIL Africa.

Simmons, P. (1997) 'Women in development: a threat to liberation' in Rahnema, Majid and Bawtree, Victoria (ed) The post-development reader, Zen Book, London: 244-255.

Smillie, I. (1997) 'NGOS and development assistance: a change in mind-set?', Third World Quarterly, 18 (3): 563-577.

Smillie, I. and Hailey, J. (2001) Managing for change: leadership, strategy \& management in Asian NGOs, London: Earthscan.

Smillie, I. and Helmich, H. (1993) Non-governmental organisations and governments: stakeholders for development, Paris: OECD. 
Smillie, I. and Helmich, H. (1999) Stakeholders in Development: Issues in Government and NGO Relationships, Paris: OECD.

Smith, A. (2000) Adult Education and Development in Central and Northern Ghana, Project completion report to DFID, SIL UK/DFID.

Sohal, A. S., Perry, M. and Pratt, T. (1998) 'Developing partnerships and networks: learning from practices in Australia' in Technovation, 18, 4: 245-251.

Søren, H. and Aaby, P. (ed) (1981) Is God American? : an anthropological perspective on the missionary work of the Summer Institute of Linguistics, Copenhagen: International work group for indigenous affairs/Survival International.

Sperling, D. (2001) Public-private technology R\&D partnerships: lessons from US partnership for a new generation of vehicles, Transport Policy, 8: 247-256. Starkey, K. (ed)(1996) How organizations learn, London: International Thomson Business Press.

Stata, R. (1996) 'Organizational learning: the key to management innovation' in $\mathrm{K}$, Starkey, (ed) How organizations learn, London: International Thomson Business Press.

Stone, D. (2001) 'The "knowledge bank" and the global development network', Paper presented in The $6^{\text {th }}$ Oxford International Conference on Education and Development on Knowledge, values and policy, September 2001. UK.

Streek, W. and Schmitter, P. (1991) 'Community, market, state-and association? The prospective contribution of interest governance to social order', in $\mathrm{G}$, Thompson, J. Frances, R. Levačić, and J. Mitchell, Markets, hierarchies and networks: The coordination of social life, London: The Open University/Sage Publication.

Sumi, K. (1989) ODA Enjyo no genjyutu (ODA: Reality of Aid), Tokyo: Iwanami.

Takahashi, C. (2000/2002) Fieldnotes, Ghana.

Takahashi, M.(2001) Memo on UK-Japan joint learning meeting mission 高橋基樹 (神戸大学) UK-Japan joint learning meeting missionについての団長所感、2001May

Tayeb, M. (2000) International business partnership: issues and concerns, New York: Palgrabe.

Taylor, M. (2000) 'Communities in the lead: power, organisational capacity and social capital', Urban Studies, 37, No. 5-6: 1019-1035. 
Taylor, S. J. and Bogdan, R. (1998) Introduction to qualitative research methods: a guidebook and resource, 3rd edition New York: John Wiley\&Sons, Inc.

The Ministry of Education, Ghana (2000) 'The Ministry of Education' [online] http://www.ghana.edu.gh/present/moeAgencies.html

The Republic of Ghana (2002) 'Economy' [online] http://www.ghana.gov.gh/aboutdetails.asp?pid=16

Thomas, A. (2000) 'Development as practice in a liberal capitalist world', Journal of International Development, 12: 773-787.

Thompson, G., Frances, J., Levačić, R and Mitchell, J. (1991) 'Introduction' in G. Thompson, J. Frances, R. Levačić, and J. Mitchell, Markets, hierarchies and networks: The coordination of social life, London: The Open University/Sage Publication.

Titmus, C. J. (1989) 'Comparative studies in adult and lifelong education', C. J. Titmus, (ed.) Lifelong education for adults: an international handbook, Oxford: Pergamon.

Todaro, M. (1997) Economic Development, Sixth edition, London: Longman.

Twumasi, P. A. (1986) A system's approach in restructuring Ghanaian society, paper presented at the $15^{\text {th }}$ Annual sociological conference, Legon May 1986.

UN (no date) 'The UN in brief: how the UN works' http://www.un.org/Overview/brief1.html.

UNDP (1996) Human Development Report, New York: Oxford University Press.

UNICEF (2000) Country Statistics, [online] www.unicef.org/statis.

Van Brabant, K. (1997) Organisational and institutional learning in the humanitarian sector opening the dialogue: a discussion paper for the active learning network on accountability and performance in humanitarian assistance, London: ODI.

Van der Heijden, H. (1987) 'The reconciliation of NGO autonomy, program integrity and operational effectiveness with accountability to donors' in World Development, 15, Supplement: 103-112.

Van Ham, H. and Koppenjan, J. (2002) 'Building public-private partnerships: assessing and managing risks in port development' in Public Management Review, 4, 1: 593-616. 
Van Rooy, A. (ed)(2001) Civil Society and the Aid Industry, London: Earthscan.

Wallace, T. (1997) 'New development agendas: changes in UK NGO policies \& procedures’ Review of African Political Economy, 71: 35-55.

Watson, K (2001) 'Comparative educational research: the need for reconceptualisation and fresh insights' in $\mathrm{K}$. Watson (ed.) Doing comparative education research: issues and problems, Oxford: Symposium Books.

Whitworth, S. (1994) 'The international planned parenthood federation', in Feminism and international relations, Macmillan press, London: 80-118.

Williams, G. (1983) Inner city policy: a partnership with the voluntary sector?, Bedford square press: London.

Woode, S. (1997) Values standards and practices in Ghanaian organisational life, Accra: Asempa Publishers.

World Bank (1998) Assessing aid: what works, what doesn't and why, A World Bank Policy research report, Oxford: Published for the World Bank/Oxford University Press.

World Bank (1999) Ghana and the World Bank, World Bank Ghana Office, Accra.

World Bank (n.d) 'Strategies-what does the World Bank want to accomplish?' [online] http://web.worldbank.org

World Bank (accessed 2001) 'Ghana Data Profile’ [online] http://devdata.worldbank.org/external/CPProfile.asp

World Bank (accessed 2002a)' poverty reduction strategies' [online] http://www.worldbank.org/poverty/strategies

World Bank (accessed 2000b) 'CDF' [online] http://www.worldbank.org/cdf/

World Bank (accessed 2000c) 'PRSP' [online]

http://www.worldbank.org/poverty/strategies/index.htm.

Wyclife (accessed Oct. 2002) [online] http://www.Wycliffe.net

Yin, K. (1994) Case study research: design and methods, London: Sage.

Young, K. (1997) 'Gender and Development' in N, Visvanathan et al (eds), The women, gender and development reader, Zed, London: 51-54.

Youngman, F. (2000) 'Imperialism, aid and adult education' in The political economy of adult education and development, Zed: London. 


\section{Appendices}

\section{Appendix 1: List of transcripts}

$\begin{array}{lll}\text { Transcript No. } & \text { Date } & \text { Position } \\ \text { Transcript 1 } & \text { Nov 01 } & \text { DFID East Kilbride Desk officer } \\ \text { Transcript 2 } & \text { Feb 01 } & \text { DFID Ghana Education Field Officer } \\ \text { Transcript 3 } & \text { Jan 01 } & \text { DFID/BHC (G) } \\ \text { Transcript 4 } & \text { Aug 01 } & \text { SIL (UK) senior staff } \\ \text { Transcript 5 } & \text { Aug 01 } & \text { SL (UK) senior Staff } \\ \text { Transcript 6 } & \text { Nov 00 } & \text { SIL (UK) volunteer group } \\ \text { Transcript 7 } & \text { Nov 00 } & \text { SIL (UK) volunteer } \\ \text { Transcript 8 } & \text { Nov 00 } & \text { GILLBT Director (G) } \\ \text { Transcript 9 } & \text { Nov 00 } & \text { GILLBT Former Director (G) } \\ \text { Transcript 10 } & \text { Nov 00 } & \text { GLLBT Associate director language project } \\ \text { Transcript 11 } & \text { Nov 00 } & \text { GILLBT Literacy Consultant } \\ \text { Transcript 12 } & \text { Nov 00 } & \text { GILLBT Accountant in Literacy and Development } \\ & & \text { Dept. (G) } \\ \text { Transcript 13 } & \text { Nov 00 } & \text { GLLBT Literacy and Development Co-ordinator (G) } \\ \text { Transcript 14 } & \text { Nov 00 } & \text { GILLBT Secretary of Literacy and Development Dept. } \\ & & \text { (G) } \\ \text { Transcript 15 } & \text { Nov 00 } & \text { GILLBT Project Funding Officer } \\ \text { Transcript 16 } & \text { Nov 00 } & \text { GLLBT Regional co-ordinator of programme in } \\ \text { Transcript 17 } & \text { Nov 00 } & \text { Dagbani Literacy Project (G) } \\ \text { Transcript 18 } & \text { Nov. 00 } & \text { Project (G) } \\ \text { Transcript 19 } & \text { Nov. 00 } & \text { GLLBT Accountant in Dagbani Literacy and Material officer (G) } \\ \text { Transcript 20 } & \text { Nov. 00 } & \text { GLLBT (Accra), Financial operations manager } \\ \text { Transcript 21 } & \text { Nov.00 } & \text { GLLBT Regional co-ordinator meeting } \\ & & \end{array}$




\begin{tabular}{|c|c|c|}
\hline Transcript 22 & Nov. 00 & GILLBT Anufo literacy project \\
\hline \multirow[t]{2}{*}{ Transcript 23} & Nov. 00 & Action Aid (Ghana) \\
\hline & & Chereponi Development Programme Officer (G) \\
\hline \multirow[t]{2}{*}{ Transcript 24} & Nov.00 & Action Aid (Ghana) \\
\hline & & Chariponi, Sponsorship programme officer (G) \\
\hline Transcript 25 & Nov. 00 & School for life (Tamale) Education officer $(G)$ \\
\hline Transcript 26 & Oct. 00 & JICA (Ghana) Project officer \\
\hline Transcript 27 & Oct. 00 & JICA (Ghana) CEP co-ordinator \\
\hline Transcript 28 & Dec. 00 & JICA (Ghana) officer \\
\hline Transcript 29 & Dec. 00 & JICA (Ghana) officer \\
\hline Transcript 30 & Dec. 00 & JICA (Ghana) officer \\
\hline Transcript 31 & Jan. 00 & Embassy of Japan (Ghana) First secretary \\
\hline Transcript 32 & Jan. 00 & Embassy of Japan (Ghana) Third secretary \\
\hline Transcript 33 & Dec. 00 & PPAG Executive Director $(G)$ \\
\hline Transcript 34 & Dec. 00 & PPAG Programme Manager (G) \\
\hline Transcript 35 & Dec. 00 & PPAG Zonal office manager (G), Accra \\
\hline Transcript 36 & Dec. 00 & PPAG Regional manager (G) \\
\hline Transcript 37 & Dec. 00 & PPAG Evaluation and Research Unit (G) Manager \\
\hline Transcript 38 & Dec. 00 & PPAG Regional officer $(G)$ \\
\hline Transcript 39 & Jan. 00 & PPAG volunteer $(G)$ \\
\hline Transcript 40 & Jan. 00 & NFED Director (G) \\
\hline Transcript 41 & Jan. 00 & NFED officer $(G)$ \\
\hline Transcript 42 & Jan. 00 & $\begin{array}{l}\text { Ministry of Employment and Social Welfare, Chief } \\
\text { director (G) }\end{array}$ \\
\hline
\end{tabular}

Transcript 43 Jan. 00 John Hopkins' University, Centre for Communication Programs (G)

Transcript 44 Dec. 00 University of Ghana, Institute of Adult Education, Director (G)

Transcript 45 Dec. 00 University of Ghana, Institute of Adult Education, Acting Director (G)

Transcript 46 Jan. 00 GMPA Director (G)

Transcript 47 Jan. $00 \quad$ Officer at Ministry of Health (G) 
Transcript 48 Jan. $00 \quad$ National Population Council Secretariat, Executive Director (G)

Transcript 49 Jan. 00 Enterprise Development Network Limited, Diector (G)

Transcript 50 Nov.00 GARIBA, Development Associates

Executive Director $(\mathrm{G})$

Transcript 51 Jan. $00 \quad$ Ghana Association of Private Voluntary Organisations in Development (GAVOD) Business Manger (G)

Transcript 52 Jan. $00 \quad$ Ghana Education Collision (G)

Transcript 53 Jan. $00 \quad$ JICA (Ghana) Japan Overseas Cooperation Volunteer

Transcript 54 Nov. 00 Action Aid (Ghana) (G) Reflect co-ordinator

Transcript 55 Sep. $01 \quad$ JICA (London) officer

Transcript 56 May. 01 Institute for International Cooperation - JICA (Tokyo) Managing director

Transcript 57 Jun $01 \quad$ JICA (Tokyo) Planning and Evaluation Dept. Deputy manager

Transcript 58 Jun $01 \quad$ JICA (Tokyo) Deputy Director of Donor-coordination Division, Planning and Evaluation Dept.

Transcript 59 Jun 01 JICA (Tokyo) Country Officer, Africa division, Regional Dept.

Transcript 60 Jun $01 \quad$ JICA (Tokyo) Senior Adviser Education

Transcript 61 Jun $01 \quad$ JOICFP (Tokyo) Director, International Program Division

Transcript 62 Jun 01 JOICEF (Tokyo) Assistant programme officer, International Programme Division

Transcript 63 Aug 01 DFID (London) Senior Education Adviser

Transcript 64 Jan $01 \quad$ GLLBT (Accra) Communication Manager (G)

Transcript 65 Jan $01 \quad$ GILLBT (Accra) Guest house manager (G)

N.B.

(G) represents Ghanaian

GILLBT interviewees were located in Tamale, unless indicated as Accra. 


\section{Appendix 2 Unpublished documents of case studies}

The following documents were mainly collected during my fieldwork in Ghana. Only a few documents were cited in the text, however many of them were useful to understand selected events and processes of day-to-day work.

\section{GILLBT}

- Allan Smith (1999) 'Project completion report to DFID' October 1996 to September 1999, SIL-UK

- GILLBT '1999 Annual report Presented to the University of Ghana, Legon' 13 April 2000 (Presented by the director GILLBT)

- John C. Cairns (2000) 'Literacy and Development Assistance Project, Ghana 1997-2000 Evaluation report' Feb-Mar 2000, GLLBT

- George Maalug and Larry Yost (1991) 'Community Development Training Manual' GILLBT 1991

- $\quad$ SIL UK 'Annual report to DFID’ Oct 1998 to Sept 1999

- $\quad$ SIL/DFID Draft Proposal for 1999-2002

- GILLBT 'Report to DFID' covering Year 2 and the 3 year project

- GILBT 'GILLBT's national literacy and development forum Feb 1999'

- Minutes of Regional coordinator meeting: May 1994; April 1995; July 1997; April 1998; January 1999; April 1999; July 1999; October 1999; January 2000; April 2000, July 2000

- Job positions and qualification an the salary scale (Revised 12-July-2000)

- Report on the ESHIE teacher training workshop held from16-18 Oct 2000

- Mother Tongue (newsletter) July/Sept 2000, March/April 2000

- Report on gender workshops held for GLLBT's literacy projects in the country (1998)

- Report on women in management workshop at GIMPA (1999)

- Report on gender workshop for GILLBT project staff (07/2000)

- Brief summary of responses to GILLBT membership questioner

- DAGONBAS and DAGBANI working church groups

- MOU between SINAPI ABA TRUST and GILLBT

- GILLBT fact sheet

- GILLBT Quarterly literacy activities

- Literacy Department regional coordinators meeting

- The role of WILD in the GILLBT programme

- GILLBT Constitution and Legislative manual

- Budget details April 1999-Mar 2002: combined donors

- GILLBT orientation reading list (1995)

- Cross cultural dos and don'ts in Ghana (TICCS)

- Tear fund: outline for writing a request to Tear fund

- GILLBT SPEL summary (Sep 1999)-Draft

- Konkomba literacy and Development programme (1996) 
- GILLBT proposal Oct 1999-Sep 2002

- Volta region quarterly literacy report (April- June 2000)

- GILLBT management structure

- GILLBT report to the DFID covering year 2 of the 3 year project (1998)

- WBT-USA Development: Project sponsoring criteria

- Possible collaboration between GILLBT and NFED

Email/letter messages

- DFID requirements on right-based awareness and empowerment approach in GILLBT proposal (June 2000)

- Letter from Civil Society Unit, DFID to SIL UK on rejecting proposal (May 1999)

- Report on meeting with DFID by SIL UK (August 1998)

- Report on SIL-UK's involvement in consultation on strengthening DFID's support for Civil Society (April-Sept 1998)

- Email from SLL UK to LDP staff on Tear fund support (August - Sept 2000)

- Email from Tear fund to LDP (April 2000)

- Email from SIL UK to LDP on DFID future funding (Sept. 1998)

- Email from GLLBT staff to LDP on DFID answer (March 2000)

- Email from SIL UK to LDP on DFID application (June 2000)

NFED

- NFED Ashanti-twi primer

- NFED Manual on training of regional and district training teams (Oct 2000)

Action Aid

- Action Aid Ghana pamphlet.

- Minutes of the Action Aid Ghana national savings and credit programme review meeting held in Bawku (1998)

- National policy framework for Action Aid Ghana savings and credit scheme (1997)

- Report on the first national REFLECT practitioners forum held at Tamale (April 2000)

- Memorandum of understanding between Action Aid and the Municipal office NFED Tamale (April 2000)

- Memorandum of understanding between Action Aid and Community Action for Development (April 2000)

\section{PPAG}

- JICA/PPAG/NPC/DCE Minutes of Meeting 23/Mar 1998.

- PPAG (Apr 1999-Mar 2000) Annual report

- PPAG (Apr1998-Mar1999) Annual report

- PPAG Research-Unit (04/2000) IP2 Mid term Evaluation report 
- PPAG Research Unit (01/2000) IP2 Mid term evaluation: transcription of focus group discussion

- PPAG (Apr.16-20 ${ }^{\text {th }}$ 2000) Report on the Fifth National Conference on the IP

- PPAG (2000) Organisational map

- Nerquaye-Tetth, J (PPAG) The role of NGOs in Population management

- JICA Ghana Pamphlet

- PPAG JICA (March 2000) Project fund accountability statement for the year ended 31 March 2000.

- PPAG (1993) $2^{\text {nd }}$ National Conference on the IP

- JOICEF (2000) A guide book for the development for sustainable CBD/CBS programmes

- Population Council and PPAG (2000) An Assessment of the Community Based Distribution Programmes in Ghana

- Benneh, G (1999) An evaluation of UNFPA-Supported integrated project in Ghana: INT/96/97-techinical and managerial support (Feb-Mar 1999), National consultant

Notes and correspondences

- Comment on financial report (JICA-G CEF) to JICA-G Rep (03/2000)

- Fax message from JICA Zambia to JICA Ghana CEP on enquiry on contract with local NGO and disbursement(05/1/2000)

- Fax message from JICA-G CEP to JICA Zambia -reply (12/01/2000)

- Fax from JICA-G CEP to JICA Tokyo(?) enquiry on schedule of disbursement (12/04/2000)

- Report from CEP to Representative in JICA Ghana on IP Conference $(08 / 05 / 2000)$

- Report from CEP Ghana to JICA Africa, Middle East, Europe section in Tokyo on mid term evaluation on IP2 (02/06/2000)

- Enquiry on 1) delay on disbursement 2) IGAs for women (09/05/2000) from CEP Ghana to JICA Africa section in Tokyo

- Letter from CEP Ghana to PPAG EC about suggestions on proposals for $2^{\text {nd }}$ phase of IP2

- Note for brainstorming on planning of proposed P2 second phase written by CEP JICA-G (06/2000)

- Report written by CEP on conference on CBD agents hosted by PPAG $(28 / 07 / 2000)$

- Table on population by district in eastern region (2000 Population and Housing Census Ghana Statistical Service) (02/08/2000)

- $\quad$ PPAG IP-Phase 2 Project activity plan (Apri 2000-Mar 2001)

- Comment on IP2 Third payment written by CEP(Than) JICA-G (27/10/2000)

- $\quad$ Report from JICA-G to JICA Africa section Tokyo on IP $2^{\text {nd }}$ Quarterly report and matrix (11/2000)

- $\quad$ Proposal for IP (PPAG)

- Investigation on proposed project to CEP for 2001 (JICA-G) to JICA-T 
- Notes from progress report (04-08/1998) (PPAG)

- DAC new strategy: Assistance research report (1998) JICA. Ghana

- Ono (JICA Ghana) (15/10/2000) Integrated human development programme: programme framework and intervention strategies

\section{Others}

- UNFPA Fourth Country Programme of Assistance to Ghana Reproductive health sub-programme.

- Institute for adult education, University of Ghana, Johns Hopkins University /Centre for Communication Programmes funded by UNFPA) (10/1995) Mass media support for adult population education: a community-based multimedia approach to population communication activities.

- Ghana Social Marketing Foundation (1999/2000) Annual Report.

- Johns Hopkins University Centre for Communication Programmes school of Hygiene and public health- pamphlet.

- Population reports newsletter: No9; 15; 39;41;43;49.

- National Population Council, Ghana (1999) Statement at a press conference held on $13^{\text {th }}$ July 1999.

- National Population Council, Ghana (2000) Country Population Assessment 4.1.2. Sources of funding pp55-69.

- National Population Council, Ghana(1994) Population Policy.

- Ministry of Health, Ghana: Management arrangements for implementation of the medium term programme of work (1997-2001).

- DFID/Ministry of Health, Ghana (2000) Promoting the Participation and financing of civil society: an options appraisal study of the Ghana health sector. 


\section{Appendix 3 Map of Ghana}

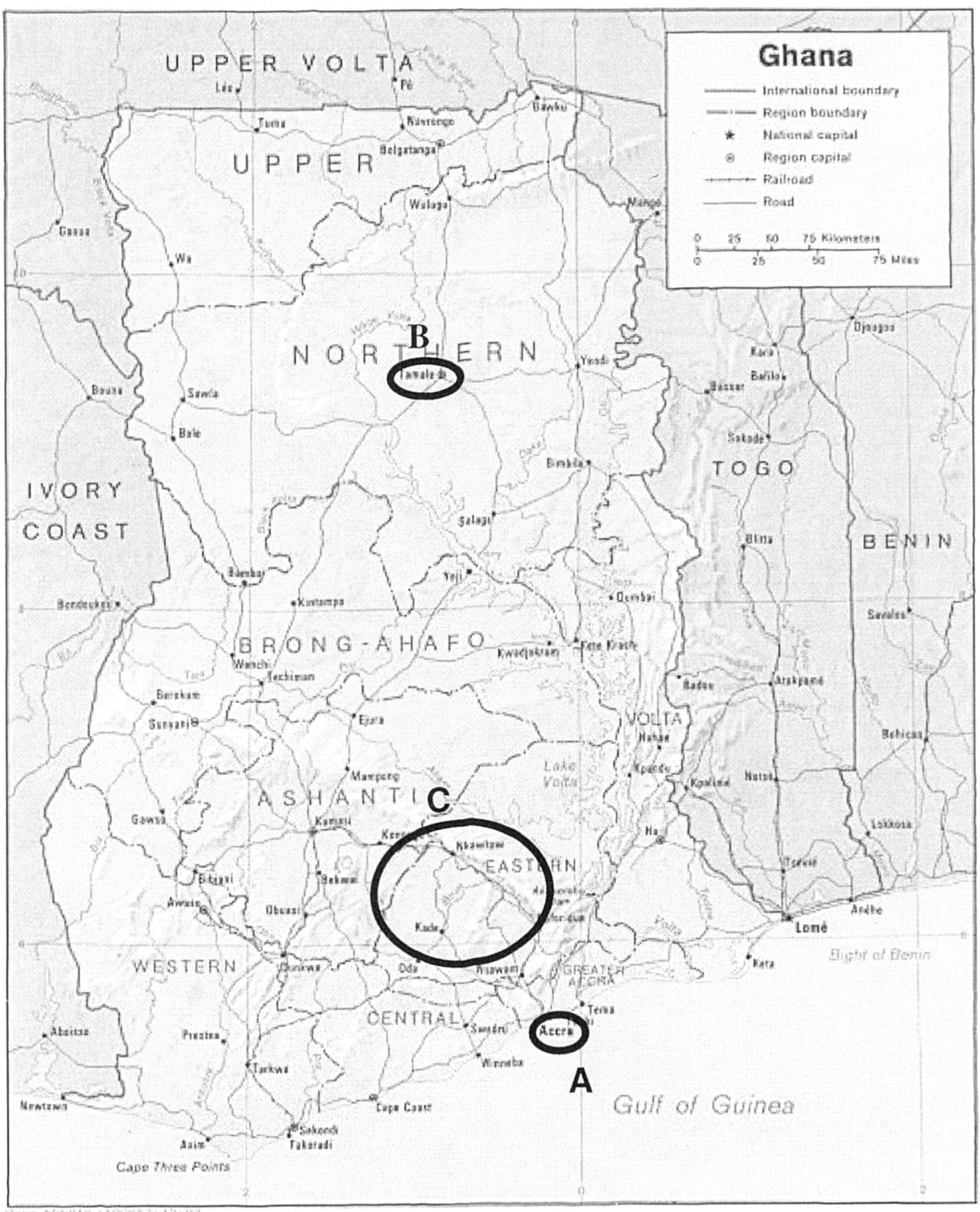

A: Accra, capital of Ghana. The headquarters of PPAG are located.

B: Tamale, The headquarters of GILLBT are located.

C: The areas where JICA-PPAG projects were implemented. 


\section{Appendix 4 GILLBT project location in Ghana}

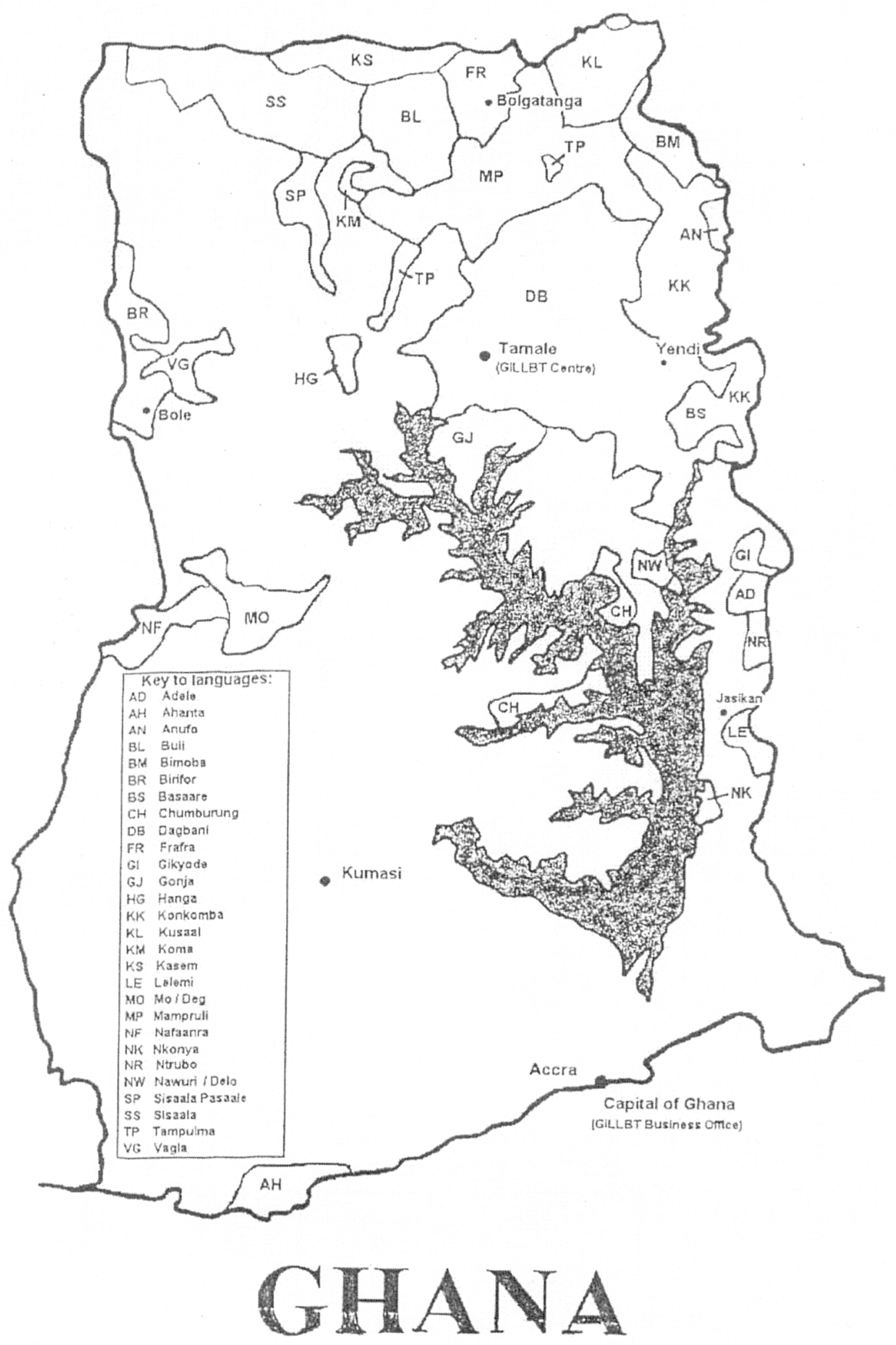




\section{Appendix 5 Structure of GILLBT's work}

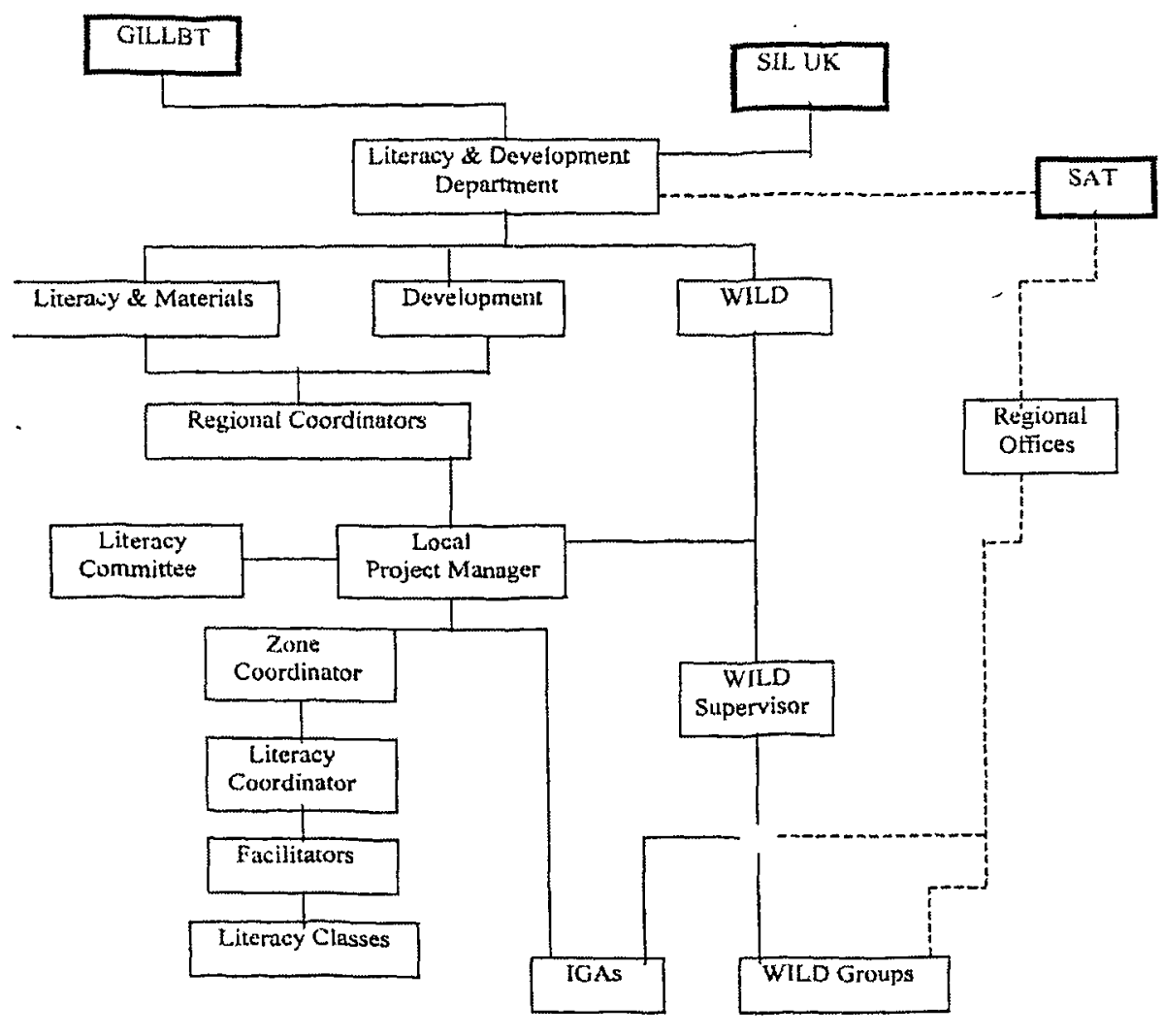

The so lid lines indicate the current structure of GILLBT's Literacy and Development Programme.

The dashed lines indicare the proposed future structure with GlLLBT working in partmership with SAT. Source: Smith (1999) 
Appendix 6 GILLBT literacy classes
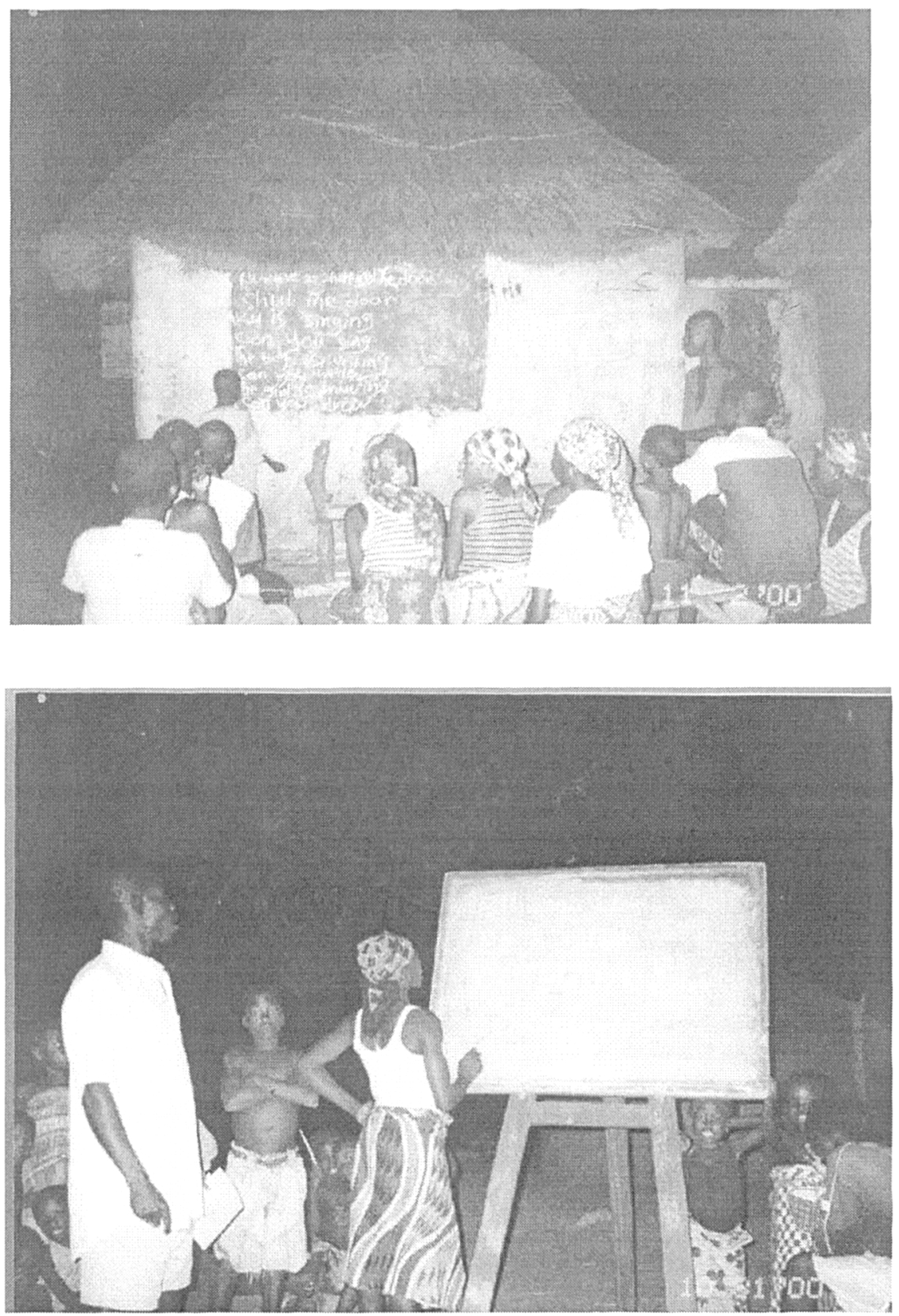


\section{Appendix 7 PPAG location in Ghana}

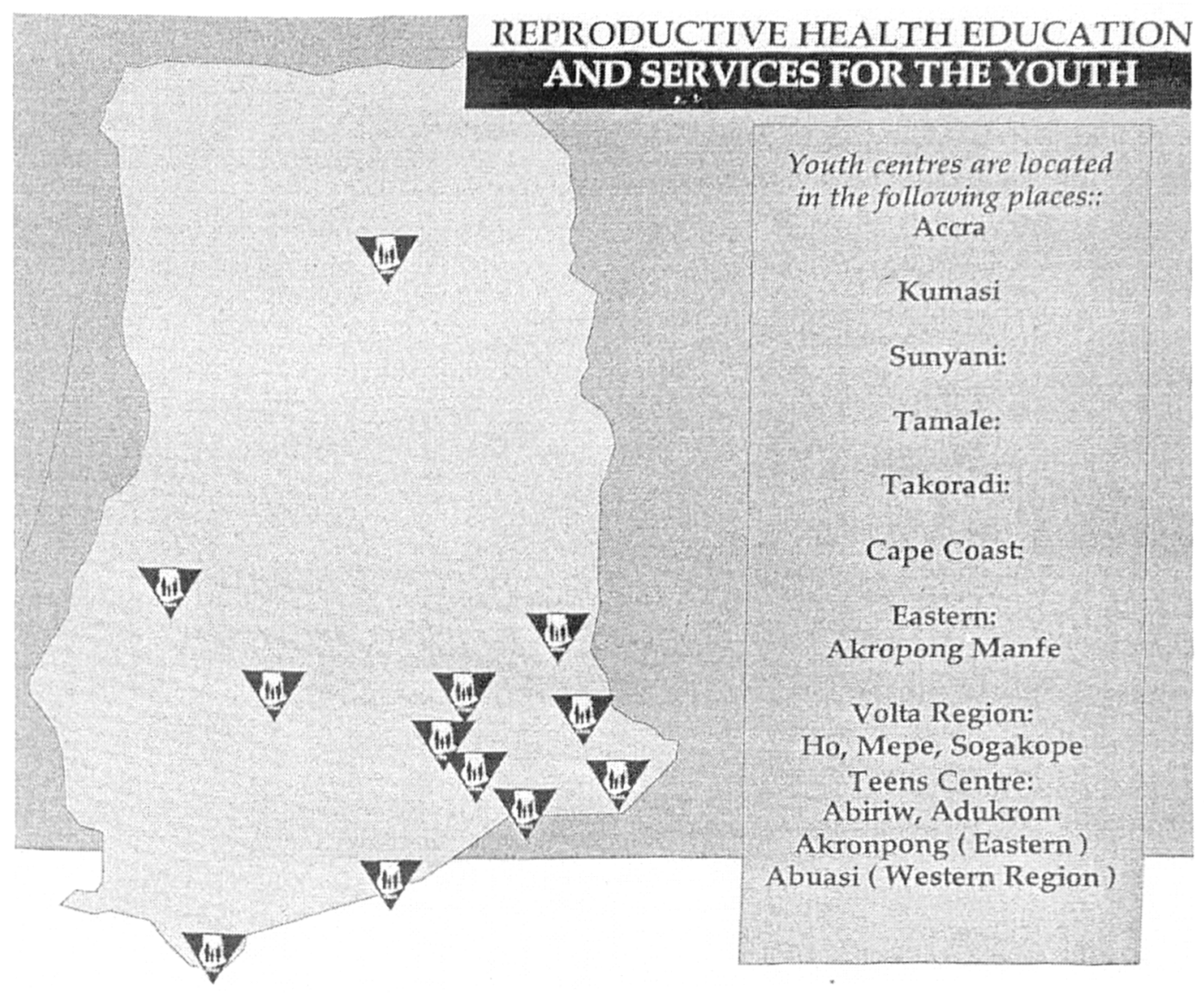




\section{Appendix 8 Organisational structure of IP management}

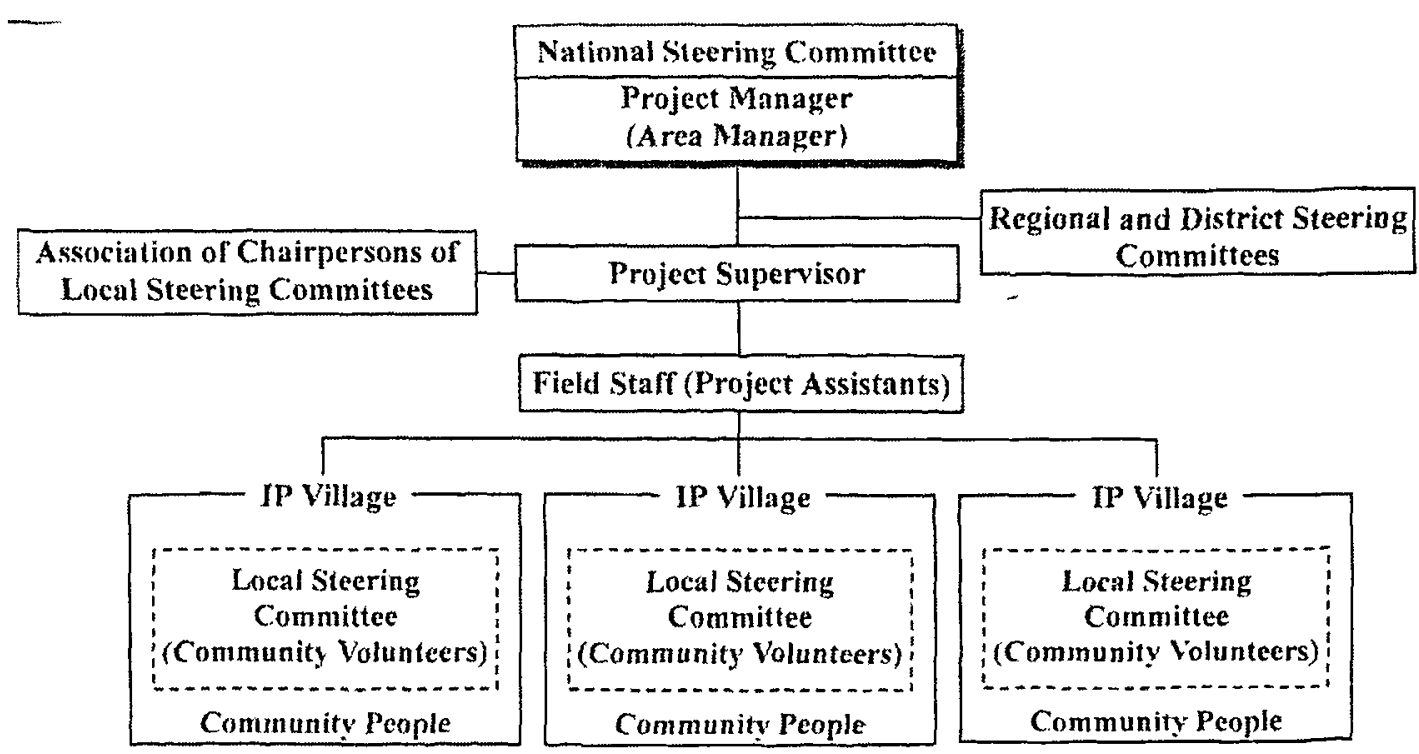

Source: JOICFP (2000)

IP: Integrated Family Planning 


\section{Appendix 9 PPAG outreach clinic}

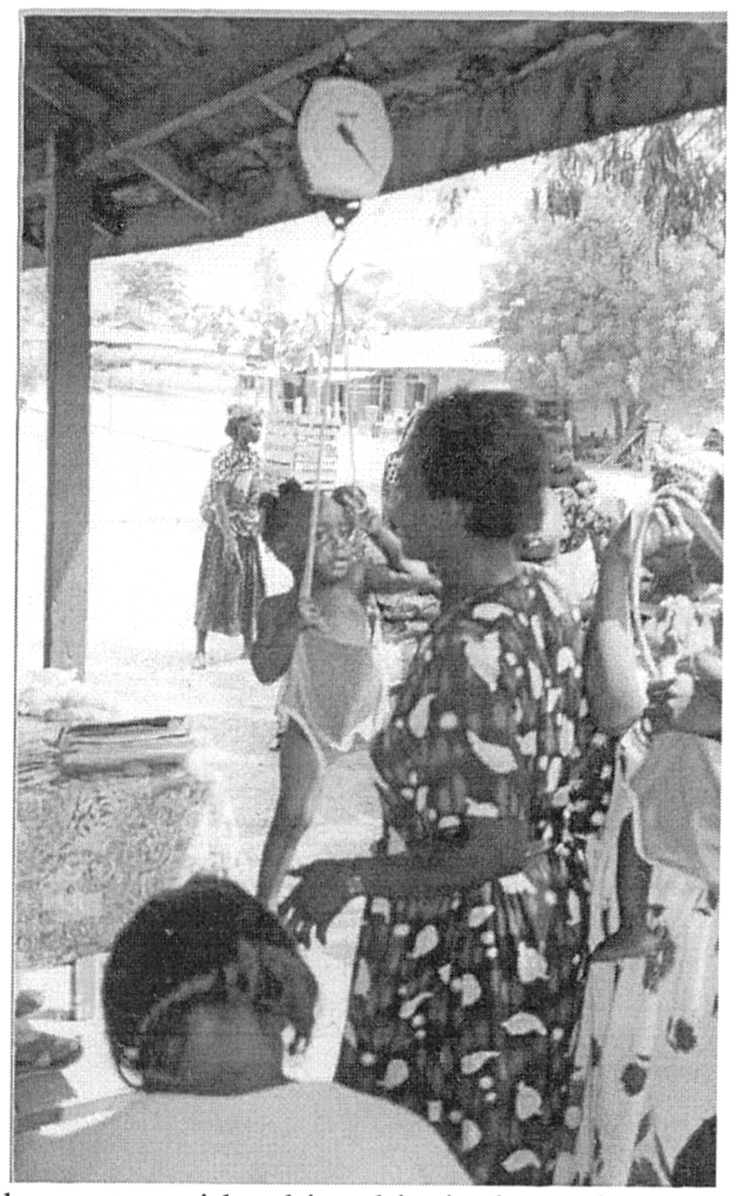

The one in the center with white shirt is the author.

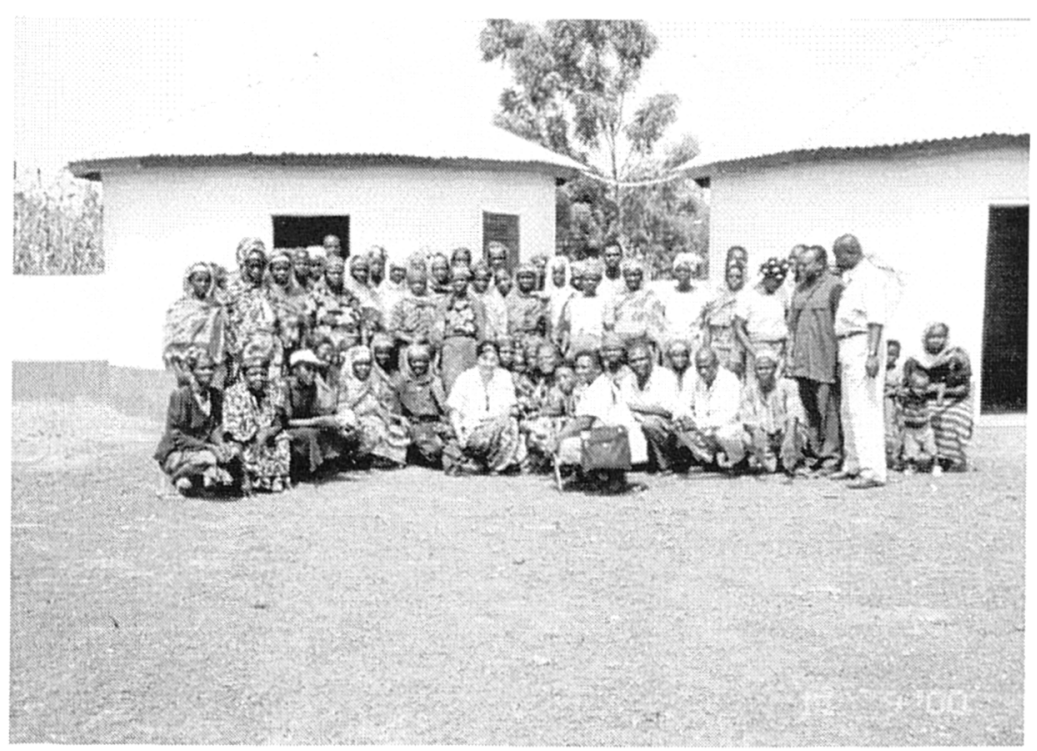

